\title{
Untersuchung des Einflusses einer modifizierten Beschilderung besonderer Bereiche in Autobahnbaustellen (Gelbe Baustelle)
}

Markus Enke

Andreas Heine

Christian Lippold

Reinhold Maier

Jörg Ortlepp

Jean Emmanuel Bakaba 


\title{
Untersuchung des Einflusses einer modifizierten Beschilderung besonderer Bereiche in Autobahnbaustellen (Gelbe Baustelle)
}

\author{
Dipl.-Ing. Markus Enke \\ Dipl.-Ing. Andreas Heine \\ Prof. Dr.-Ing. Christian Lippold \\ Prof. Dr.-Ing. Reinhold Maier \\ Dipl.-Ing. Jörg Ortlepp \\ Dr.-Ing. Jean Emmanuel Bakaba
}


Die Unfallforschung der Versicherer veröffentlicht ihre Forschungsergebnisse in den Reihen:

FS - Fahrzeugsicherheit

VI - Verkehrsinfrastruktur

VV - Verkehrsverhalten / Verkehrspsychologie

\section{Impressum:}

\section{Gesamtverband der Deutschen Versicherungswirtschaft e. V.}

\section{Unfallforschung der Versicherer}

Wilhelmstraße 43/43G, 10117 Berlin

Postfach 0802 64, 10002 Berlin

E-Mail: unfallforschung@gdv.de

Internet: www.udv.de

ISBN-Nr.: 978-3-939163-43-5

Redaktion:

Dipl.-Ing. Markus Enke, Dipl.-Ing. Andreas Heine,

Prof. Dr.-Ing. Christian Lippold, Prof. Dr.-Ing. Reinhold Maier

Dipl.-Ing. Jörg Ortlepp, Dr.-Ing. Jean Emmanuel Bakaba

Layout: Michaela Gaebel

Erschienen: 12/2011 


\section{Untersuchung des Einflusses einer modifizierten Beschilderung besonderer Bereiche in Autobahnbaustellen (Gelbe Baustelle)}
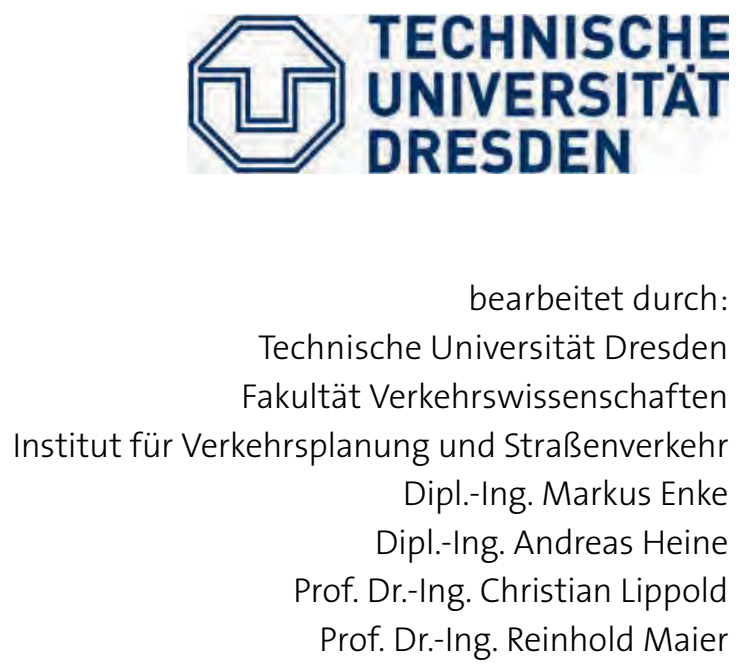

Unfallforschung der Versicherer

Bei der UDV betreut von:

Dipl.-Ing. Jörg Ortlepp 



\section{Inhalt}

1

$2 \quad$ Literaturübersicht $\quad \mathbf{8}$

2.1 Rechtliche Grundlagen 8

2.2 Leiteinrichtungen und Verkehrszeichen in Arbeitsstellen 8

2.3 Untersuchungen zur Verwendung von modifizierter Beschilderung in Autobahnbaustellen 8

2.3.1 Verkehrsablauf

2.3.2 Verkehrssicherheit

2.3.3 Empfehlungen zur Verwendung von modifizierter Beschilderung in Baustellen 9

2.4 Leit- und Warnbaken

2.5 Schlussfolgerungen für die Umsetzung in einer Untersuchungsbaustelle

$3 \quad$ Methodik und Datenerhebung

3.1 Charakteristik der Untersuchungsbaustelle 11

3.2 Durchführung 12

3.3 Gestaltung der Untersuchungsbereiche 12

3.3.1 Verkehrszeichen und Leiteinrichtungen 12

3.3.2 Annäherungsbereich (Zulaufbereich) 13

3.3.3 Baustellenende 13

3.3.4 Anschlussstelle in der Baustelle $\quad 17$

$3.4 \quad$ Datenerhebung 18

3.4.1 Nachfolgefahrten $\quad 18$

3.4.2 Querschnittsmessungen 20

$3.5 \quad$ Unfallanalyse 24

3.6 Gesamtanalyse 24

$4 \quad$ Analyse des Verkehrsablaufs $\quad 26$

$4.1 \quad$ Allgemeines 26

4.2 Annäherungsbereich und erste Verschwenkung 29

4.3 Baustellenende 34

4.4 Anschlussstelle innerhalb der Baustelle 36

4.5 Wirkung der stufenweisen Anhebung der zulässigen
Höchstgeschwindigkeit nach der Baustelle

4.6 Zusammenfassung 40

$5 \quad$ Unfallgeschehen $\quad 42$

$6 \quad$ Zusammenfassung und Empfehlungen 43 
Anhang

A Baustellenfreier Autobahnabschnitt

A.1 Freie Geschwindigkeiten auf dem baustellenfreien Autobahnabschnitt

A.2 Verkehrsablauf 50

B Untersuchungsbaustelle 53

C Liste der 3-stelligen Unfalltypen $\quad 59$

C.1 Baustellenbereich 59

C.2 Annäherungsbereich

C.3 Baustellenende 67

D Freie Geschwindigkeiten im Baustellenbereich 69

$\begin{array}{lll}\text { D.1 Methodik } & 69\end{array}$

$\begin{array}{lll}\text { D.2 Ergebnisse } & 71\end{array}$

$\begin{array}{lll}\text { D.2.1 Allgemeines } & 71\end{array}$

$\begin{array}{ll}\text { D.2.2 Annäherungsbereich } & 72\end{array}$

D.2.3 Baustellenende 80

$\begin{array}{lll}\text { D.2.4 Anschlussstelle } & 87\end{array}$

E Längsabstände $\quad 89$

E.1 Methodik $\quad 89$

$\begin{array}{lll}\text { E.2 Auswertung } & 92\end{array}$

E.3 Baustellenbeginn 93

$\begin{array}{lll}\text { E.4 Baustellenende } & 98\end{array}$

$\begin{array}{lll}\text { E.5 Anschlussstelle } & 103\end{array}$

F $\quad$ Fahrstreifenbelegung $\quad 104$

$\begin{array}{lll}\text { F.1 Baustellenbeginn } & 104\end{array}$

F.2 Baustellenende 105

G Unfallgeschehen 107 


\section{$1 \quad$ Einleitung}

Baustellen auf Autobahnen sind gegenüber störungsfreien Abschnitten auf Autobahnen nicht pauschal mit einem erhöhten Unfallrisiko verbunden. Dies zeigen die Ergebnisse einer Untersuchung von Verkehrssicherheit und -ablauf in Baustellen auf Autobahnen (QSV, 2010). Während von Veränderungen der Verkehrsführung unbeeinflusste Abschnitte innerhalb von Autobahnbaustellen eine vergleichsweise hohe Verkehrssicherheit aufweisen, stellten sich insbesondere Bereiche von Fahrstreifenverschwenkungen als Schwerpunkte von Unfällen heraus. Dabei erwiesen sich aber nicht die Überleitungen von Fahrstreifen am Beginn, sondern vielmehr solche innerhalb und vor allem am Ende der Baustellen als unfallauffällig.

Aus den Daten des Verkehrsablaufs ergab sich eine generelle Überschreitung der zulässigen Höchstgeschwindigkeiten durch einen großen Teil der erfassten Fahrzeuge. Die Geschwindigkeiten von etwa 50 \% aller Fahrzeuge (v50) lagen über den zulässigen Höchstgeschwindigkeiten. Zudem lag die Perzentilgeschwindigkeit ${ }^{1} \mathrm{v}_{85}$, als die Geschwindigkeit, die $85 \%$ aller Fahrzeuge nicht überschreiten, zwischen $10 \mathrm{~km} / \mathrm{h}$ und $20 \mathrm{~km} / \mathrm{h}$ über den zulässigen Werten. Vor allem bei Geschwindigkeitsbeschränkungen von $60 \mathrm{~km} / \mathrm{h}$ wurde dabei deutlich zu schnell gefahren. Solche niedrigen Limits werden allerdings überwiegend bei besonderen Verkehrssituationen, wie Verschwenkungen angeordnet. Besonders hier wirken sich zu hohe Geschwindigkeiten negativ auf die Verkehrssicherheit aus. Diese Bereiche werden häufig bestimmt durch enge Kurvenradien und dadurch geringe Seitenabstände zwischen den Fahrzeugen. Hohe Geschwindigkeiten und möglicherweise damit im Zusammenhang stehendes häufigeres Überfahren der Mittellinien können Unfälle durch seitliches Streifen begünstigen. Auch können die zu hohen Geschwindigkeiten im Zusammenhang mit den Verschwenkungen der Fahrstreifen zum Kontrollverlust über das Fahrzeug führen.
Die Analysen der Unfallarten bestätigten diese Szenarien. Unfälle durch Abkommen von der Fahrbahn und seitliches Streifen von in gleicher Richtung fahrenden Fahrzeugen bestimmen den Großteil des Unfallgeschehens in Bereichen von Fahrstreifenverschwenkungen. Zwar werden diese Bereiche durch Beschilderung angekündigt, dennoch lässt sich offenbar dadurch nicht ausreichend auf die Unfallgefahr hinweisen.

Demzufolge werden in dieser Untersuchung vorhandene Ansätze zur Verdeutlichung von verkehrsunsicheren Bereichen innerhalb von Autobahnbaustellen durch farblich hervorgehobene Gestaltung von Verkehrszeichen und Leiteinrichtungen aufgegriffen und auf deren Einfluss überprüft. In die Analyse soll neben dem Verkehrsablauf auch das Unfallgeschehen des Bauzeitraums einbezogen werden. Durch die kurzen Untersuchungszeiträume waren geringen Unfallzahlen in den Bereichen zu erwarten. Eine Unfallauswertung auf Basis dieser Einzelereignisse ist nicht möglich. Jedoch können die Angaben zu Hergang und Charakteristik der Unfälle Indizien für die Wirkung der Maßnahmen liefern. 


\section{Literaturübersicht}

\subsection{Rechtliche Grundlagen}

Die maßgebende Rechtsgrundlage für die Sicherung von Arbeitsstellen sind $\S 45$ Abs. 1 und 2 der StraßenverkehrsOrdnung StVO sowie die zugehörigen Verwaltungsvorschrift zur Straßenverkehrs-Ordnung (VwV-StVO). Demnach erfolgt die Sicherung von Arbeitsstellen nach den RSA (1995) und den Zusätzlichen technischen Vertragsbedingungen und Richtlinien für die Sicherungsarbeiten an Arbeitsstellen an Straßen (ZTV-SA, 1997). Nach $\S 43$ Absatz 3 Anlage 4 Abschnitt 1 der VwV-StVO (2009) erfolgt

„... die Sicherung von Arbeitsstellen und der Einsatz von Absperrgeräten nach den Richtlinien für die Sicherung von Arbeitsstellen an Straßen (RSA), die das für Verkehr zuständige Bundesministerium im Einvernehmen mit den zuständigen obersten Landesbehörden im Verkehrsblatt bekannt gibt."

In den RSA 95 ist die Anordnung der Beschilderungen geregelt. Zudem enthalten die RSA Regelpläne für die Einrichtung von Arbeitsstellen. Diese stellen Standardsituationen dar und sind den jeweiligen örtlichen Gegebenheiten der Arbeitsstellen anzupassen (Schönborn / Schulte, 1999).

Für die Ausgestaltung und Beschaffenheit der Absperrgeräte gelten die Vorschriften der $\S \S 39$ bis 43 Nummer II, III 1 bis 7 (Rn. 5 ff.) VwV-StVO (2009). Absperrgeräte sind demnach mindestens voll retroreflektierend auszuführen ( $\$ 43$ Absatz 3 Anlage 4 Abschnitt 1 der VwVStVO, 2009).

Leitplatten bzw. Leitbaken (Zeichen 605) werden bei Hindernissen auf oder neben der Fahrbahn so angeordnet, dass die Streifen nach der Seite fallen, auf der an dem Hindernis vorbeizufahren ist ( $\$ 43$ Absatz 3 Anlage $4 \mathrm{Ab}$ schnitt 2 und 3 der VwV-StVO, 2009). Die Ausführungen der VwV-StVO (Stand 2009) beziehen sich lediglich auf die Anordnung von Schraffenbaken.

\subsection{Leiteinrichtungen und Verkehrs- zeichen in Arbeitsstellen}

Verkehrszeichen und -leiteinrichtungen können mit unterschiedlichen rückstrahlenden Eigenschaften ausgeführt werden. Zweckmäßige Bauarten sind in den Hinweisen für die Wahl der Bauart Verkehrszeichen und Verkehrsleiteinrichtungen hinsichtlich ihrer lichttechnischen Eigenschaften (HWBV, 2001) angegeben. Nach $\S 39$ bis $\S 43$ StVO ist die Beschilderung von Baustellen auf Autobahnen aber mindestens in Bauart 2 (Typ-2-Reflexfolie nach DIN 67520-2, 1994) auszuführen.

Vor allem Verschwenkungen von Fahrstreifen oder deren Überleitung auf die Fahrbahn der Gegenrichtung erfordern eine visuelle Führung der Fahrzeugführer. Beim Einsatz von Leitbaken mit Schraffen zur visuellen Führung in diesen Bereichen müssen die rot/weißen-Streifen zum Verkehrsbereich hin abfallen. Dadurch soll auf die Seite, auf der das Fahrzeug vorbeifahren soll, hingewiesen werden. Zur Verdeutlichung besonders kritischer Situationen, wie Inselspitzen oder der Teilung von Fahrstreifen (3+1-Verkehrsführung), werden die größeren Warnbaken eingesetzt. Leit- und Warnbaken müssen den Technischen Lieferbedingungen TL-Leitbaken (1997) entsprechen.

\subsection{Untersuchungen zur Verwendung von modifizierter Beschilderung in Autobahnbaustellen}

\subsubsection{Verkehrsablauf}

Aus bisherigen Untersuchungen zum Hervorheben der Beschilderung in Baustellenbereichen ergibt sich eine positive Wirkung auf das Fahrverhalten. Beim Einsatz im Annäherungsbereich sowie der ersten Überleitung stellten sich geringere Überschreitungen der zulässigen Höchstgeschwindigkeiten sowie ein früherer Beginn der Geschwindigkeitsreduzierung ein.

BAIER et al. (2006) betrachteten in ihren Untersuchungen ausschließlich die Zulaufbereiche von Baustellen auf Autobahnen (im Weiteren: Annäherungsbereiche). Im Fokus 
stand der Bereich unmittelbar vor der Verschwenkung am Baustellenbeginn. In einer Vorher-/ Nachher-Untersuchung wurde der Einfluss fluoreszierender Materialien (selektivgelb) bei den Leiteinrichtungen auf den Verkehrsablauf überprüft. Demnach führte eine Ausstattung des Zulaufbereichs mit selektivgelben Verkehrszeichen und Leiteinrichtungen zu geringeren Überschreitungen der zulässigen Höchstgeschwindigkeiten. Dieser Effekt stellte sich vor allem bei Dämmerung, aber auch am Tag ein. Ein Einfluss auf das Fahrverhalten bei Nacht, sowohl positiv als auch negativ (Blenden), konnte nicht nachgewiesen werden. Im Vergleich zur Standardbeschilderung trat der Verzögerungsprozess der Geschwindigkeiten früher ein und führte damit im relevanten Bereich $100 \mathrm{~m}$ vor der Überleitung der Fahrstreifen zu geringeren Geschwindigkeiten. Ebenfalls positiv hat sich eine rot / selektivgelbe Gestaltung der Leitbaken auf das Geschwindigkeitsverhalten in der Überleitung selbst ausgewirkt.

In den jeweils vierwöchigen Untersuchungszeiträumen konnten keine Gewöhnungseffekte nachgewiesen werden.

\subsubsection{Verkehrssicherheit}

Da der Untersuchung von BAIER et al. (2006) nur ein kurzer Beobachtungszeitraum von jeweils 4 Wochen für jede Gestaltungsvariante zu Grunde lag, konnten keine Aussagen zum Unfallgeschehen vorgenommen werden.

\subsubsection{Empfehlungen zur Verwendung von modifizierter Beschilderung in Baustellen}

Aus den Erkenntnissen wird in BAIER et al. (2006) die Empfehlung eines dosierten Einsatzes von Leiteinrichtungen in selektivgelber Ausführung am Beginn von Autobahnbaustellen gegeben. So lässt sich ein besser auf die Situation angepasstes Verhalten der Verkehrsteilnehmer erreichen. Bei der Umsetzung sollte selektivgelbe - fluoreszierend grünlich-gelbe - Typ-3-Reflexfolie zum Einsatz kommen. Es sollte sich auf Bereiche konzentriert werden, die dem Kraftfahrer eine besondere Fahraufgabe abverlangen. Dazu zählen in Autobahnbaustellen vor allem die Überleitungen der Fahrstreifen auf eine andere Fahrbahn.
Abgeraten wird dagegen von der Modifizierung mehrfarbiger Verkehrszeichen (Z 123 Arbeitsstelle; Z 274 - Beschränkung der zulässigen Höchstgeschwindigkeit) mit selektivgelber Reflexfolie. Demnach ist aus einer Umgestaltung dieser Zeichen keine Verbesserung der Erkennbarkeit zu erwarten.

Der regelmäßige Einsatz selektivgelber Beschilderung und Leiteinrichtungen erfordert eine entsprechende Änderung der StVO, sowie des Katalogs der Verkehrszeichen (VzKat) und RSA. Bei der Umsetzung sind inkonsistente Ausführungen, insbesondere die Vermischung von Schraffen- und Pfeilbaken, ebenso zu vermeiden wie der inflationäre Gebrauch von fluoreszierenden selektivgelben Materialien bei der Gestaltung von Leiteinrichtungen und Verkehrszeichen. Nur so können die Effekte auf die Verkehrssicherheit, die aus der Besonderheit dieser Gestaltung resultieren, möglichst langfristig aufrecht erhalten werden. Nach BAIER et al. (2006) sollten die selektivgelbe Beschilderung den Bereichen von Überleitungen der Fahrstreifen auf die Fahrbahn der Gegenrichtung am Beginn von Autobahnbaustellen vorbehalten bleiben.

Eine Übertragung der Erkenntnisse auch auf Arbeitsstellen kürzerer Dauer ist nach BAIER et al. (2006) möglich, sollte allerdings zunächst in weiterführenden Wirksamkeitsuntersuchungen überprüft werden.

\subsection{Leit- und Warnbaken}

Neben dem Einsatz fluoreszierender Materialien wurde in BAIER et al. (2006) auch der Unterschied von Schraffen und Pfeilbaken auf das Fahrverhalten überprüft. Dies geschah im Rahmen eines Feldversuchs auf einem abgesperrten Autobahnabschnitt mit 34 Probanden. Diese mussten einen Parcours durchfahren, der in seiner Gestaltung der Verkehrsführung in einer Autobahnbaustelle entsprach. Bei verschiedenen Durchfahrten wurden jeweils die Beschilderung und Leiteinrichtungen verändert. Im Ergebnis zeigte sich, dass unter sonst gleichen Bedingungen Pfeilbaken gegenüber Schraffenbaken eine verbesserte Leitwirkung aufwiesen. 
Die Verwendung von Schraffen- und Pfeilbaken in Bereichen von Fahrstreifen $\neg$ verschwenkungen in Baustellen auf Autobahnen war auch Gegenstand von weiteren Untersuchung (u.a. Meseberg, 1997 und SCHULZE et al., 2010). Diese Untersuchungen haben gezeigt, dass nur einer Minderheit von befragten Personen die richtungsweisende Funktion von Schraffenbaken bekannt war. Die visuell leitende Wirkung von Schraffenbaken gründet dabei vor allem in der Anordnung mehrerer Baken und weniger im dargestellten Schildinhalt. Dagegen überwiegt bei der Verwendung von Pfeilbaken die Information des Schildinhalts.

In der Untersuchung von SCHULZE et al. (2010) mussten die Probanden eine Strecke befahren, auf welcher in einem Zeitabstand von $3 \mathrm{~s}$ vor dem Fahrzeug die Baken (einzeln) auftauchten und dabei jeweils nach der richtigen Seite ausweichen. Bei diesem Test wurde beim Auftauchen von Pfeilbaken hochsignifikant häufiger an der richtigen Seite vorbeigefahren (87,8\%) als an Schraffenbaken (70,2 \%). Zudem erfolgten die Reaktionen beim Auftauchen von Pfeilbaken deutlich schneller.

Nach SCHULZE erhöhen Pfeilbaken bei sorgfältiger Verwendung und exakter Aufstellung die Wahrscheinlichkeit des korrekten Verhaltens und gewährleistet ein schnelleres Erkennen der Richtungsanweisung.

\subsection{Schlussfolgerungen für die Umsetzung in einer Untersuchungsbaustelle}

Aus der bisherigen Untersuchung zur gelben Beschilderung von Baustellenbereichen geht hervor, dass diese durchaus eine positive Wirkung auf das Fahrverhalten haben kann. Beim Einsatz im Annäherungsbereich sowie der ersten Überleitung stellten sich geringere Überschreitungen der zulässigen Höchstgeschwindigkeiten sowie ein früherer Beginn der Geschwindigkeitsreduzierung ein.

Im Mittelpunkt der Betrachtungen stehen die folgenden Bereiche, welche sich in Untersuchungen als besonders unsicher für die Verkehrsteilnehmer erwiesen hatten:
- Verschwenkungen und Überleitungen von Fahrstreifen

- Behelfsanschlussstellen

- Baustellenende (Verschwenkungen und Überleitungen von Fahrstreifen).

Für eine Veränderung des Fahrverhaltens in einzelnen Bereichen müssen alle damit im Zusammenhang stehenden Verkehrszeichen in das Gestaltungskonzept einbezogen werden. Beispielsweise sind für die Fahrstreifenverschwenkung am Beginn der Baustelle alle mit der besonderen Verkehrsführung im Zusammenhang stehenden Verkehrszeichen entsprechend zu modifizieren:

- alle Überleitungs-/Fahrstreifen- bzw. Verschwenkungstafeln (600 m, 400 m, 200 m),

- die speziell für den Bereich der Fahrstreifenverschwenkung geltende Reduzierung der zulässigen Höchstgeschwindigkeit und

- zugehörige Leit-/Warnbaken

Die in der Vergangenheit durchgeführten Untersuchungen zur Wirkung unterschiedlicher (farblicher) Gestaltungen von Verkehrszeichen auf die Erkennbarkeit bei Tag und Nacht (BAIER et al., 2006) bilden die Grundlage für die Wahl selektivgelber (fluoreszierend grünlich-gelben) Typ-3-Reflexfolie bei der Modifizierung der Beschilderung. Da dieser Empfehlung umfassende vergleichende Untersuchungen zur Wirkung und Erkennbarkeit der Beschilderung zu Grunde lagen, wird diese hier übernommen.

Bei der Ausstattung einer optimierten Baustelle sollten außerdem möglichst Pfeilbaken verwendet werden, da diese bessere Leitfunktionen bewiesen haben als Schraffenbaken. 


\section{Methodik und Datenerhebung}

\subsection{Charakteristik der Untersuchungsbaustelle}

Der Autobahnabschnitt mit der Untersuchungsbaustelle weist in Fahrtrichtung 1 zwei Fahrstreifen und in Fahrtrichtung 2 drei Fahrstreifen auf. Zudem befindet sich im betrachteten Bereich eine Anschlussstelle. Im Durchschnitt wird der Bereich täglich von 37.663 Fahrzeugen (am Querschnitt) mit einem Schwerverkehrsanteil von etwa $14,5 \%$ befahren. Das entspricht 5.438 Lkw pro Tag.

Die untersuchte Autobahnbaustelle erstreckte sich über eine Länge von ca. 6,2 km und einen Zeitraum von etwa 4 Monaten und wurde mit einer 4+0-Verkehrsführung betrieben.

Dazu erfolgte im Annäherungsbereich der Fahrtrichtung 2 eine Reduzierung der von drei auf zwei Fahrstreifen. Die Fahrstreifen der Gegenrichtung (Fahrtrichtung 1), auf deren Fahrbahn sich das Baufeld befand, wurden im Baustellenbereich auf der Fahrbahn der Richtung 2 geführt. Für die Anpassung der Verkehrsführung war somit in Fahrtrichtung 1 eine Überleitung der Fahrstreifen auf die Gegenfahrbahn (Abbildung 3 3) und für Fahrtrichtung 2 eine Fahrstreifenverschwenkung (Abbildung 3-2) erforderlich. Innerhalb der Baustelle wurden die Fahrstreifen beider Fahrtrichtungen unverändert (ohne weitere Verschwenkungen) mit konstanten Breiten geführt. Nach den Verschwenkungen am Ende der Baustelle erfolgte eine gestufte Anhebung der zulässigen Höchstgeschwindigkeit. Dazu wurde am Baustellenende die Beschränkung zunächst auf $100 \mathrm{~km} / \mathrm{h}$ angehoben. Nach weiteren 500 $\mathrm{m}$ erfolgte dann die Freigabe (Fahrtrichtung 1) bzw. abschließende Anhebung auf 130 km/h (Fahrtrichtung 2).

Für den Bauzeitraum wurde die Anschlussstelle innerhalb der Baustelle für die Fahrtrichtung 1 gesperrt. In Fahrtrichtung 2 wurde die Möglichkeit des Ein- und Ausfahrens von der Autobahn aufrecht erhalten. Während die Längen der Beschleunigungs- bzw. Verzögerungsstreifen gegenüber dem baustellenfreien Zustand unverändert blieben, wurden deren Breiten verringert.
Nach der Einrichtung blieb die Verkehrsführung über den gesamten Bauzeitraum unverändert. Verkehrszeichen und Leiteinrichtungen wurden zunächst nach den Vorgaben der RSA (1995) mit herkömmlichen weißen (Hintergrundfarbe) und Typ-2-Reflexfolie ausgestattet. Damit entsprach die Baustelle im Vorher-Zeitraum der üblichen Gestaltung einer Autobahnbaustelle in Deutschland. Nach vier Wochen erfolgte die Umgestaltung der für die Untersuchung relevanten Beschilderung unter Verwendung von selektivgelber Typ-3-Reflexfolie.

Durch den Vorher-/Nachher-Vergleich lässt sich die Wirkung der Veränderung der Beschilderung direkt bestimmen. Die Baustellenbeschilderung unterscheidet sich zwischen den beiden Zeiträumen durch die Farbgebung der Hintergrundtafeln und die Verwendung einer Folie mit verbesserten Reflexionseigenschaften für ausgewählte Verkehrszeichen. Gegenstand der Untersuchung sind Zeichen, die im direkten Zusammenhang mit besonderen Verkehrssituationen stehen:

\section{- Beginn der Baustelle \\ (Schlussabschnitt des Annäherungsbereichs) \\ - Fahrstreifenverschwenkungen \\ - Anschlussstellen innerhalb der Baustelle}

Auf diese Bereiche konzentrieren sich die weiteren Untersuchungen.

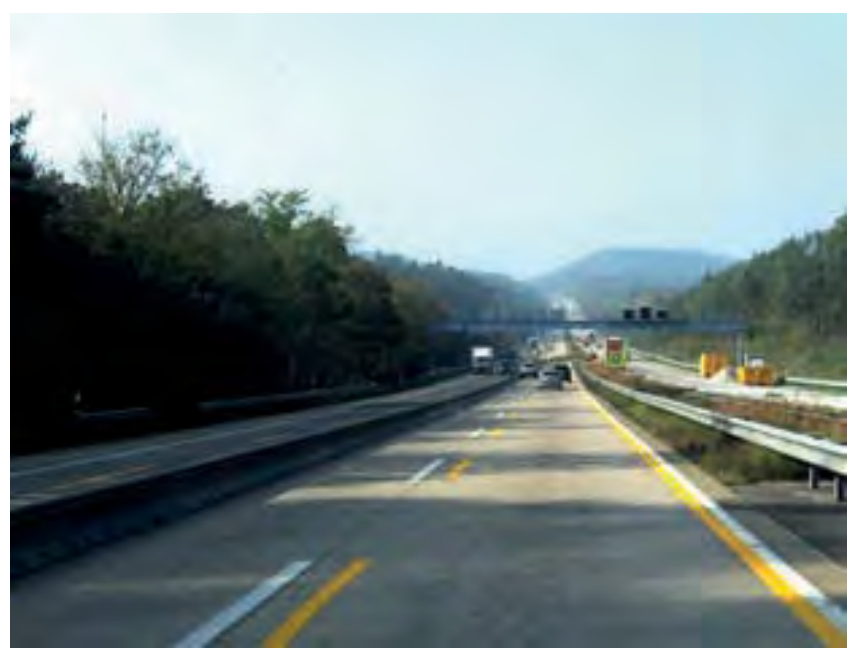

Abbildung 3-1:

4+0-Verkehrsführung im Baustelleninnenbereich 


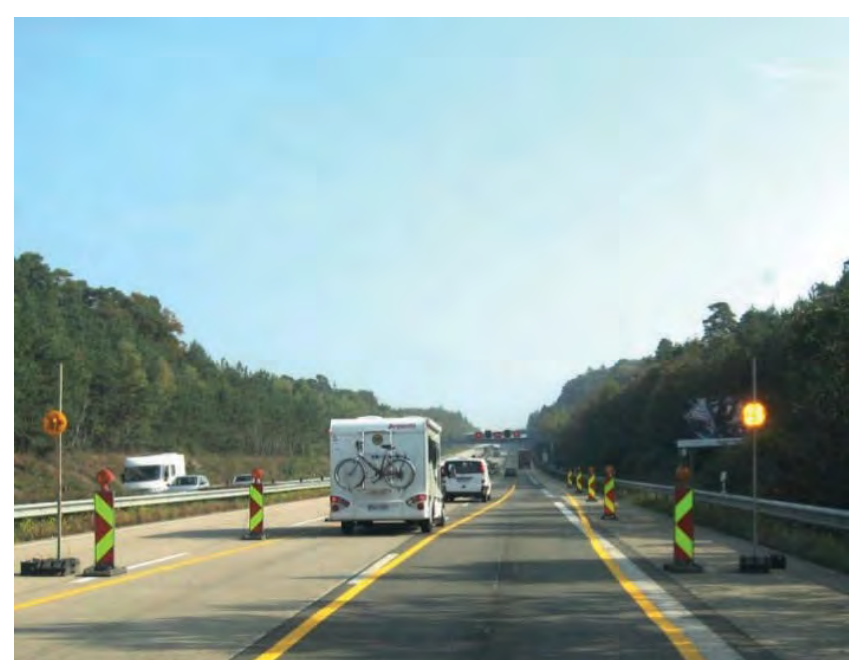

Abbildung 3-2:

Verschwenkung von Fahrstreifen am Baustellenbeginn (selektivgelbe Beschilderung)

\subsection{Durchführung}

Die in 3.1 beschriebene Baustelle wurde zunächst nach RSA 95 mit Verkehrszeichen und Leiteinrichtungen in der Ausführung mit weißer Typ-2-Reflexfolie ausgeführt. Im Nachher-Zeitraum wurden zuvor festgelegte Verkehrszeichen und Leiteinrichtungen entsprechend den Erkenntnissen in Kapitel 2.5 durch die Verwendung selektivgelber Hintergrundtafeln bzw. einer Gestaltung der sonst weißen Flächen mit selektivgelber Folie modifiziert. In beiden Betrachtungsphasen erfolgten folgende Untersuchungen:

- Nachfolgefahrten zur Bestimmung des Geschwindigkeitsverlaufs über den gesamten Baustellenbereich und

- lokale Messungen an mehreren festgelegten Querschnitten.

- Darüber hinaus werden die im Bauzeitraum aufgetretenen Unfälle ausgewertet. Der Schwerpunkt der abschließenden Gesamtanalyse liegt aber auf der Bewertung des Einflusses der verkehrstechnischen Modifizierung auf das Fahrverhalten. Aufgrund des kurzen Betrachtungszeitraums lassen sich aus der Analyse des Unfallgeschehens keine gesicherten Erkenntnisse ableiten.

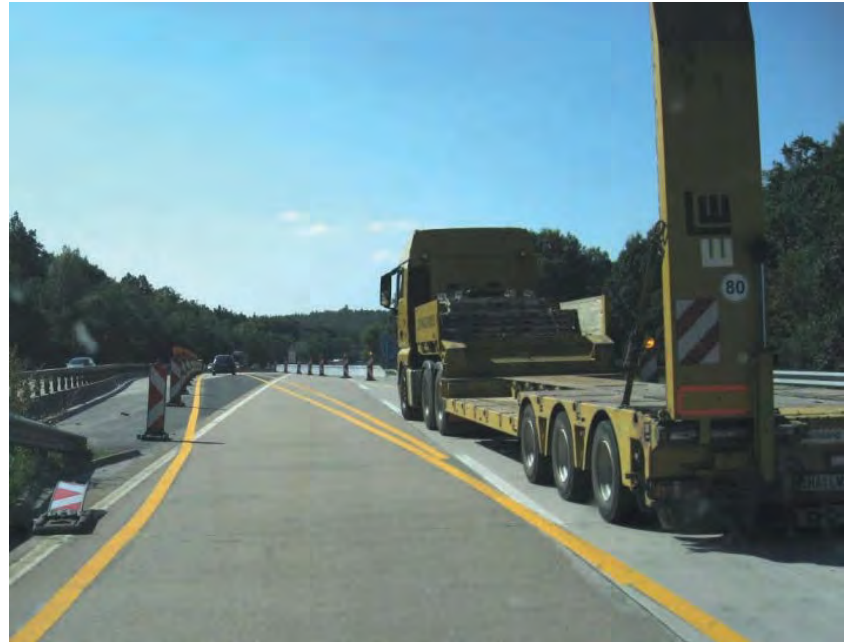

Abbildung 3-3:

Überleitung von Fahrstreifen am Baustellenbeginn (weiße Beschilderung)

\subsection{Gestaltung der Untersuchungsbereiche}

\subsubsection{Verkehrszeichen und Leiteinrichtungen}

Es wurde zu Beginn vermutet, dass der besondere Einfluss punktuell eingesetzter optisch auffällig gestalteter Verkehrszeichen auf das Fahrverhalten der Fahrzeugführer. Grundlage für diese These waren die Erkenntnisse der in Kapitel 2.3 beschriebenen Untersuchungen zum Einfluss modifizierter Verkehrszeichen und Leiteinrichtungen.

Vor dem Hintergrund der in OSV (2010) festgestellten Defizite der Verkehrssicherheit in Bereichen von Fahrstreifenverschwenkungen und den dort gleichzeitig beobachteten Überschreitungen der zulässigen Höchstgeschwindigkeiten müssen Möglichkeiten gefunden werden, den Verkehrsteilnehmer auf die besondere Gefahrensituation hinzuweisen. An Anschlussstellen innerhalb von Baustellen, die auch häufig Ort von Unfällen sind, wird das Problem weniger in den Geschwindigkeiten als vielmehr im rechtzeitigen Erkennen der Situation gesehen.

Aus den Erkenntnissen der Literaturanalyse ließ sich durch das Hervorheben der mit der direkten Gefahrensituation in Zusammenhang stehenden Beschilderung und 
Leiteinrichtung ein positiver Effekt auf das Fahrverhalten und damit die Sicherheit ableiten. Die aus BAIER et al. (2006) resultierende Gestaltung von Leiteinrichtungen mit positiven Effekten auf das Fahrverhalten wurde für die vorliegende Untersuchung übernommen. Die in die Modifizierung einbezogenen Verkehrszeichen und Leiteinrichtungen sind in Tabelle 3-1 dargestellt.

Grundsätzlich wurden die Richtzeichen modifiziert, welche den Fahrer auf eine Situation, die eine aktive Änderung des Fahrverhaltens erfordert, hinweisen. Diese sind: - Zeichen 505-11 (Überleitungstafel),

- Zeichen 505-13 (Verschwenkungstafel) und

- Zeichen 531-11 (Einengungstafel).

Nach BAIER sollten die weißen Flächen bei mehrfarbigen Verkehrszeichen (z.B. Zeichen 274) nicht durch selektivgelbe Folien ersetzt werden. Andererseits stellen die Fahrgeschwindigkeiten aber ein maßgebliches Problem bei der Verkehrssicherheit vor allem der Fahrstreifenverschwenkungen dar. Zudem besteht die Gefahr, dass die Zeichen zur Beschränkung der zulässigen Höchstgeschwindigkeit optisch hinter die farblich hervorgehobenen Fahrstreifentafeln und -baken zurücktreten. Aus diesem Grund wurden in der Untersuchung alle mit den untersuchten Bereichen im direkten Zusammenhang stehenden Zeichen der Geschwindigkeitsbeschränkung (Zeichen 274) im Zustand "Gelb“ auf einer selektivgelb ausgeführten Hintergrundtafel dargestellt (Tabelle 3 1). Das (mehrfarbige) Verkehrszeichen selbst wurde nicht modifiziert. Gleiches gilt für das Zeichen 276 (Überholverbot). Dieses wurde auch mit selektivgelb ausgeführter Hintergrundtafel ausgestattet, $\mathrm{da}$ in den vorangegangenen Untersuchungen (QSV, 2010) insbesondere in den Fahrstreifenverschwenkungen und Anschlussstellen sehr viele Unfälle durch seitliches Streifen von in gleicher Richtung fahrenden Fahrzeugen auftraten.

Die aus der Literatur hervorgehende bessere Leitwirkung von Pfeilbaken insbesondere in Bereichen von Fahrstreifenverschwenkungen (SCHULZE et al., 2010) war Grundlage für deren Einsatz in den Bereichen der Verschwenkungen und Überleitungen von Fahrstreifen. Diese wurden ebenfalls entsprechend der Untersuchungsphase in rot / weiß bzw. rot / selektivgelb ausgeführt.

\subsubsection{Annäherungsbereich (Zulaufbereich)}

Annäherungsbereiche sind in erster Linie durch die schrittweise Verringerung der zulässigen Höchstgeschwindigkeit vom baustellenfreien Autobahnabschnitt hin zur Baustelle geprägt. Diese Verringerung erfolgt in Schritten zu $20 \mathrm{~km} / \mathrm{h}$ und Abständen von $200 \mathrm{~m}$. An den Untersuchungsbaustellen begann die Reduzierung $600 \mathrm{~m}$ vor der ersten Veränderung der Verkehrsführung. In Fahrtrichtung 1 war das die Überleitung der Fahrstreifen auf die Gegenfahrbahn und in Fahrtrichtung 2 die baustellenbedingte Reduzierung der Anzahl der Fahrstreifen.

Die charakteristischen Merkmale der beiden untersuchten Annäherungsbereiche sind in Tabelle 3-2 gegenübergestellt.

Am Beispiel des Annäherungsbereichs der Fahrtrichtung 1 sind in Tabelle 3-3 die Gestaltungen der beiden untersuchten Beschilderungszustände gegenübergestellt. Aus dieser Darstellung wird die in Kapitel 3.3.1 beschriebene Umsetzung der selektivgelben Beschilderung in Abhängigkeit des Bereiches deutlich. Neben den Überleitungstafeln erhielt auch das Zeichen 274-56 $\left(v_{z u l}=60 \mathrm{~km} / \mathrm{h}\right)$ aufgrund seines direkten Bezugs zur besonderen Fahrsituation eine gelbe Hintergrundtafel.

Als Besonderheit ist im Bereich des Baustellenbeginns eine Streckenbeeinflussungsanlage zu nennen, deren Anzeige nicht von der veränderten Farbgebung betroffen war. Die Anzeigen (Z 274) richteten sich nach der jeweiligen Regelung an der Stelle des Portals.

\subsubsection{Baustellenende}

Am Ende der Baustelle wurden die Fahrstreifen der Richtung 1 wieder auf ihre Fahrbahn übergeleitet. Die Überleitung wurde $200 \mathrm{~m}$ zuvor durch Zeichen 505-11 (Überleitungstafel) angekündigt. Etwa $100 \mathrm{~m}$ vor diesem Bereich erfolgte zudem eine Reduzierung der zulässigen Höchstgeschwindigkeit von $80 \mathrm{~km} / \mathrm{h}$ auf $60 \mathrm{~km} / \mathrm{h}$. In Fahrtrichtung 2 erfolgte am Baustellenende lediglich die Aufweitung der verengten Fahrstreifenbreiten und im Nachlauf die Addition des eingezogenen dritten Fahrstreifens. 
Tabelle 3-1:

Verkehrszeichen und Leiteinrichtungen in weißer und gelber Ausführung

\begin{tabular}{|c|c|c|}
\hline Zeichen & $\begin{array}{l}\text { Weiße Beschilderung } \\
\text { Vorher-Zeitraum }\end{array}$ & $\begin{array}{l}\text { Gelbe Beschilderung } \\
\text { Nachher-Zeitraum }\end{array}$ \\
\hline 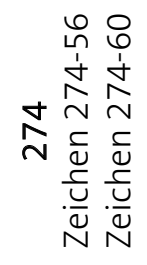 & & \\
\hline 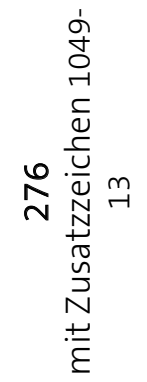 & & \\
\hline 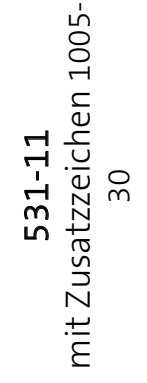 & \begin{tabular}{|c|}
$\begin{array}{c}\text { Reißverschluss } \\
\text { erst in } 400 \mathrm{~m}\end{array}$ \\
\end{tabular} & \begin{tabular}{|c|}
$\begin{array}{c}\text { Reißverschluss } \\
\text { erst in } 400 \mathrm{~m}\end{array}$ \\
\end{tabular} \\
\hline 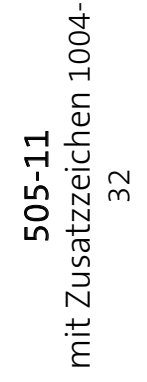 & $200 \mathrm{~m}$ & $\frac{\Lambda}{200 \mathrm{~m}}$ \\
\hline 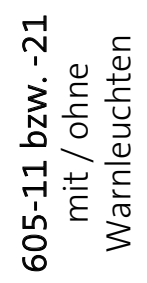 & & \\
\hline
\end{tabular}


Tabelle 3-2:

Merkmale der Gestaltung der Annäherungsbereiche der beiden Fahrtrichtungen

\begin{tabular}{|l|c|c|}
\hline & Fahrtrichtung 1 & Fahrtrichtung 2 \\
\hline $\begin{array}{l}\text { Anzahl der zuführenden } \\
\text { Fahrstreifen }\end{array}$ & 2 & 3 \\
\hline $\begin{array}{l}\text { Abstand der Reduzierung der } \\
\text { zulässigen Höchstgeschwin- } \\
\text { digkeit }\end{array}$ & $700 \mathrm{~m}, 500 \mathrm{~m}, 100 \mathrm{~m}$ & $1.200 \mathrm{~m}, 1.000 \mathrm{~m}, 800 \mathrm{~m}$ \\
\hline Fahrstreifenreduzierung & nein & $500 \mathrm{~m}$ \\
\hline $\begin{array}{l}\text { Art der Verschwenkung der } \\
\text { Fahrstreifen }\end{array}$ & Überleitung & Verschwenkung \\
\hline $\begin{array}{l}\text { Zulässige } \\
\text { Höchstgeschwindigkeit am } \\
\text { Beginn der } \\
\text { Fahrstreifenverschwenkung }\end{array}$ & $60 \mathrm{~km} / \mathrm{h}$ & $80 \mathrm{~km} /$ \\
\hline $\begin{array}{l}\text { Beginn der Leitbaken } \\
\text { und Verringerung der } \\
\text { Fahrstreifenbreiten) }\end{array}$ & $400 \mathrm{~m}$ & $500 \mathrm{~m}$ \\
\hline $\begin{array}{l}\text { Messquerschnitte-Abstand } \\
\text { vor Baustellenbeginn }\end{array}$ & $500 \mathrm{~m}, 200 \mathrm{~m}, 0 \mathrm{~m}$ & $1.100 \mathrm{~m}, 500 \mathrm{~m}, 200 \mathrm{~m}, 0 \mathrm{~m}$ \\
\hline $\begin{array}{l}\text { Anmerkung: Alle Längenmaße beziehen sich jeweils auf den Abstand zur Fahrstreifenver- } \\
\text { schwenkung am Beginn der Baustelle }\end{array}$ & \\
\hline
\end{tabular}

Da nur in Fahrtrichtung 1 eine Veränderung der Verkehrsführung vorlag, die eine verstärkte Aufmerksamkeit des Fahrers erforderte, wurde hier im NachherZeitraum folgende Beschilderung gelb ausgestattet (Tabelle 3-4):

- Zeichen 274-56 - Beschränkung der zulässigen Höchstgeschwindigkeit auf $60 \mathrm{~km} / \mathrm{h}$

- Zeichen 505-11 - Überleitungstafeln und

- Zeichen 605-11/21 - Leitbaken in der Fahrstreifenüberleitung.
Im Anschluss an die Überleitung der Fahrstreifen in Fahrtrichtung 1 bzw. die Fahrstreifenaddition in Fahrtrichtung 2 am Ende der Baustelle erfolgte zunächst eine Anhebung der zulässigen Höchstgeschwindigkeit auf 100 km/h (Zeichen 274-60) und erst $500 \mathrm{~m}$ nach der Baustelle die Aufhebung aller baustellenbezogenen Einschränkungen.

Dabei wird wiederum die Hintergrundtafel des Zeichens 274-60 und damit gleichzeitig auch die des Zeichens 277 (Überholverbot für Kraftfahrzeuge mit einem zulässigen Gesamtgewicht über 3,5 t) mit selektivgelber Typ-3-Reflexfolie ausgestattet. 
Tabelle 3-3:

Gestaltung der Annäherungsbereiche (hier: Fahrtrichtung 1)

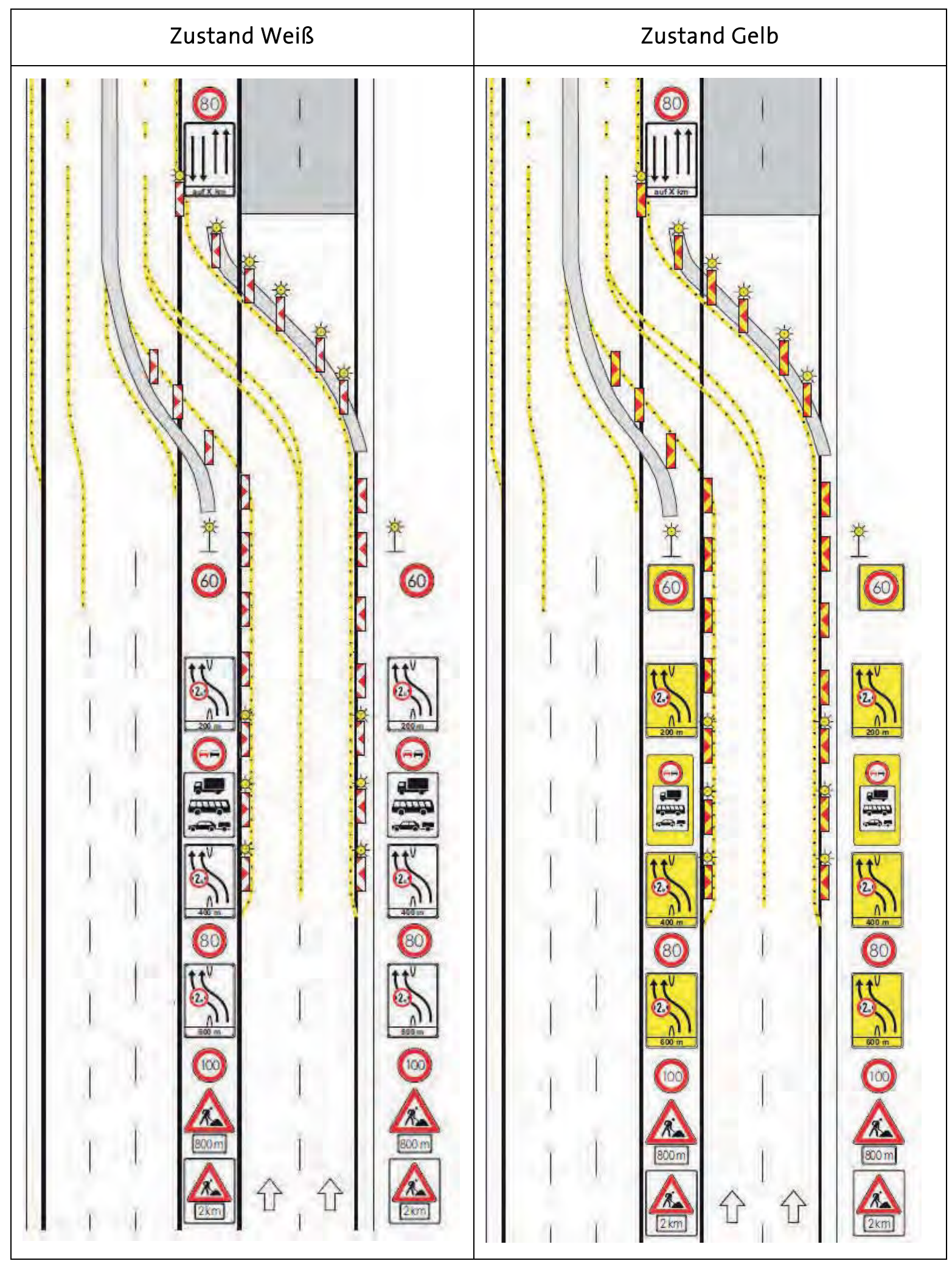


Tabelle 3-4

Gestaltung der Überleitung am Baustellenende in Fahrtrichtung 1

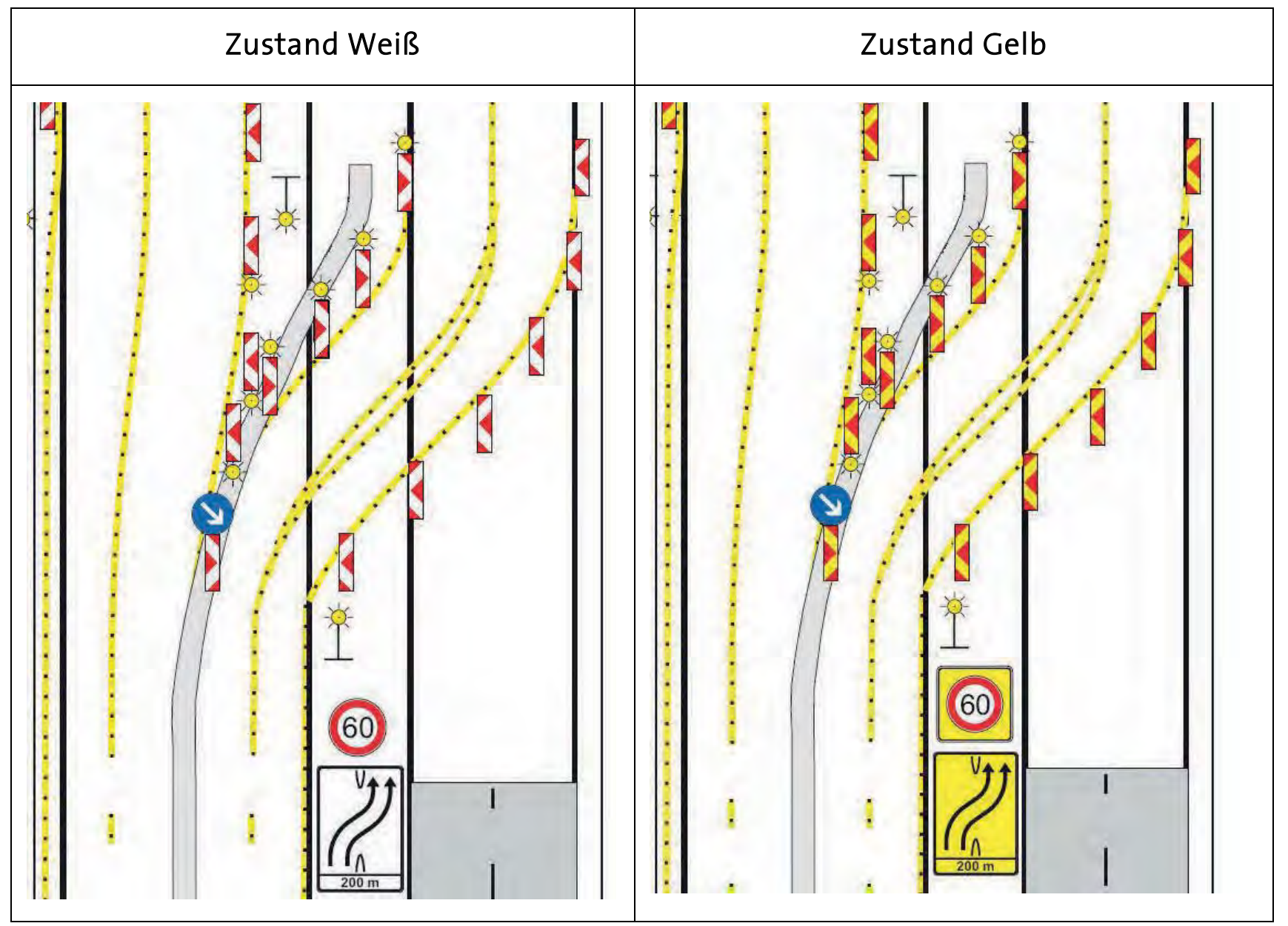

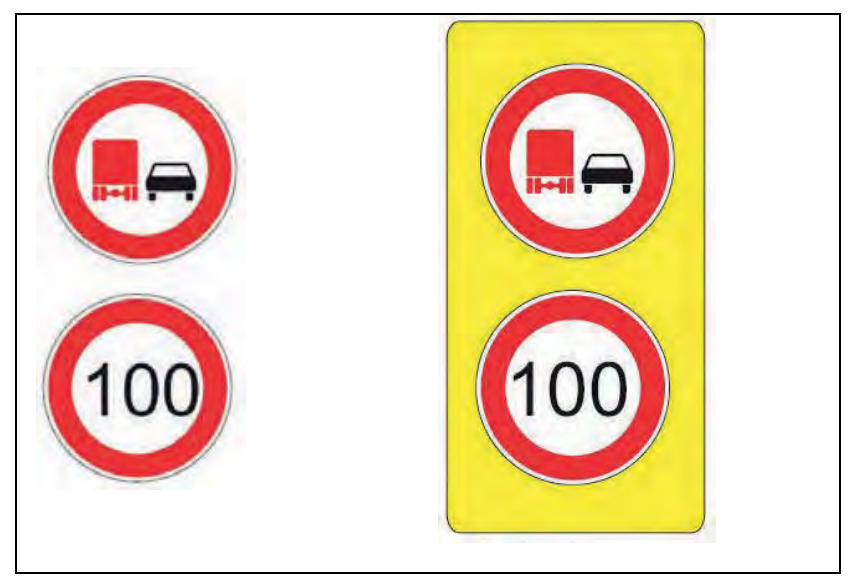

Abbildung 3-4:

Beginn der stufenweisen Anhebung der zulässigen Höchstgeschwindigkeit am Ende der Baustelle

\subsubsection{Anschlussstelle in der Baustelle}

Im Mittelpunkt der Untersuchung des Verkehrsablaufs im Bereich der Anschlussstelle standen die Geschwindigkeiten und Längsabstände sowie die Fahrstreifenbelegung auf der Hauptfahrbahn. Unfälle durch seitliches Streifen (Unfallart 3) beschreiben im gesamten Bereich jeweils den größten Anteil des Unfallgeschehens. Vor allem an den Ausfahrten ereignen sich überdurchschnittlich viele Unfälle durch Zusammenstöße mit Fahrzeugen, die vorausfahren oder warten (Unfallart 2). An Einfahrten treten zudem Unfälle mit einfahrenden Fahrzeugen auf. Über 30 \% der Unfälle an Einfahrten mit Beschleunigungsstreifen ereignen sich durch Abkommen von der Fahrbahn. 
Die Gründe für die Häufung von Unfällen durch Auffahren auf ein vorausfahrendes Fahrzeug werden vor allem im plötzlichen Abbremsen eines vorausfahrenden Fahrzeugs im Zusammenhang mit dem Ein- oder Ausfahren von der Autobahn gesehen. Es wird vermutet, dass Fahrzeuge, welche die Anschlussstelle nicht nutzen wollen, die besondere Verkehrssituation und damit verbundene mögliche Veränderungen im Verkehrsablauf (Abbremsen, Einfahrende Fahrzeuge) nicht rechtzeitig erkennen. Aber auch einfahrende Fahrzeuge müssen deutlicher auf die besondere Situation verkürzter oder nicht vorhandener Beschleunigungsstreifen hingewiesen werden.

Aus diesem Grund wurde auch die Beschilderung der Anschlussstelle vor allem im Bereich der Einfahrt hervorgehoben. Neben der Beschränkung der zulässigen Höchstgeschwindigkeit auf $60 \mathrm{~km} / \mathrm{h}$ im Bereich der Einfahrt wurden auch die Zeichen 123 in der Zufahrt mit einer selektivgelben Hintergrundtafel ausgeführt (Abbildung 3-5). Außerdem wurden die Leitbaken der Einfahrt sowie die Warnbake im Bereich der Abteilung des Ausfahrstreifens in die Modifizierung der Beschilderung einbezogen. Aufgrund der häufig auftretenden Unfälle durch seitliches Streifen wurde zudem die Wiederholung des Zei-

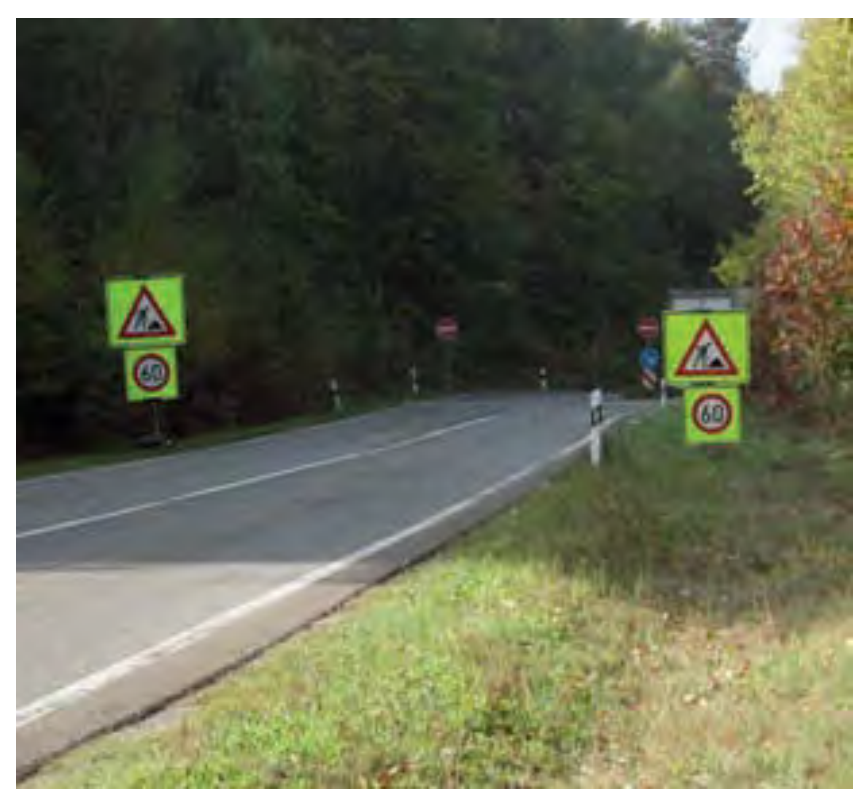

Abbildung 3-5:

Hervorgehobene Beschilderung in der Einfahrrampe zur Autobahn chens 276 mit Zusatzzeichen 1049-13 mit einer gelben Hintergrundtafel versehen.

Die Verringerung der Geschwindigkeitsbeschränkung auf der Hauptfahrbahn soll den langsameren einfahrenden Fahrzeugen die Möglichkeiten zum Einfahren in den Hauptstrom verbessern. Die Wiederholung des Zeichens 277 (Überholverbot für Kraftfahrzeuge mit einem zulässigen Gesamtgewicht über 3,5 t) direkt vor der Einfahrt soll den Verkehrsteilnehmer nochmals auf die Gefahrensituation hinweisen (Tabelle 3-5).

\subsection{Datenerhebung}

\subsubsection{Nachfolgefahrten}

Das Fahrverhalten von Kraftfahrern lässt sich generell durch das Geschwindigkeits-, Beschleunigungs- und Spurverhalten beschreiben. Für Baustellen sind besonders die Geschwindigkeit, die häufig im Zusammenhang mit der Schwere von Unfällen steht, und die Spurposition der Fahrzeuge in verengten Fahrstreifen und Verschwenkungsbereichen maßgebend.

Zur Beurteilung des Geschwindigkeitsverlaufs in allen Abschnitten der Baustelle wurden Geschwindigkeitsprofile anhand von Nachfolgefahrten erstellt. Dabei wurden einzelne, zufällig ausgewählte Fahrer mit einem Fahrzeug, das mit dem GPS-Gerät "GARMIN GPSmap 60Cx“ ausgerüstet war (Tabelle 3 7), in konstantem Abstand verfolgt. Die Nachfolgefahrten orientierten sich an zufällig ausgewählten Fahrzeugen, konnten jedoch nicht in stets konstantem zeitlich-räumlichem Abstand durchgeführt werden. Vielmehr erfolgte ein „Mitschwimmen“ im Verkehr, wobei z.B. Fahrstreifenwechsel und damit verbundene Beschleunigungs- und Verzögerungsvorgänge nach Verkehrslage angepasst werden. Verließ ein verfolgtes Fahrzeug die Baustelle an einer Ausfahrt oder entfernte es sich aus dem Sichtbereich, weil der Verkehr ein Folgen unmöglich machte, wurde ein anderes Fahrzeug zur Orientierung genutzt und die Fahrt fortgesetzt. Insbesondere im Annäherungsbereich vor und im Nachbereich hinter der Baustelle war jedoch das Nachfolgen in konstantem räumlich-zeitlichen Abstand in aller Regel möglich, so dass 
Tabelle 3-5:

Gestaltung der Anschlussstelle im Baustellenbereich in Fahrtrichtung 2

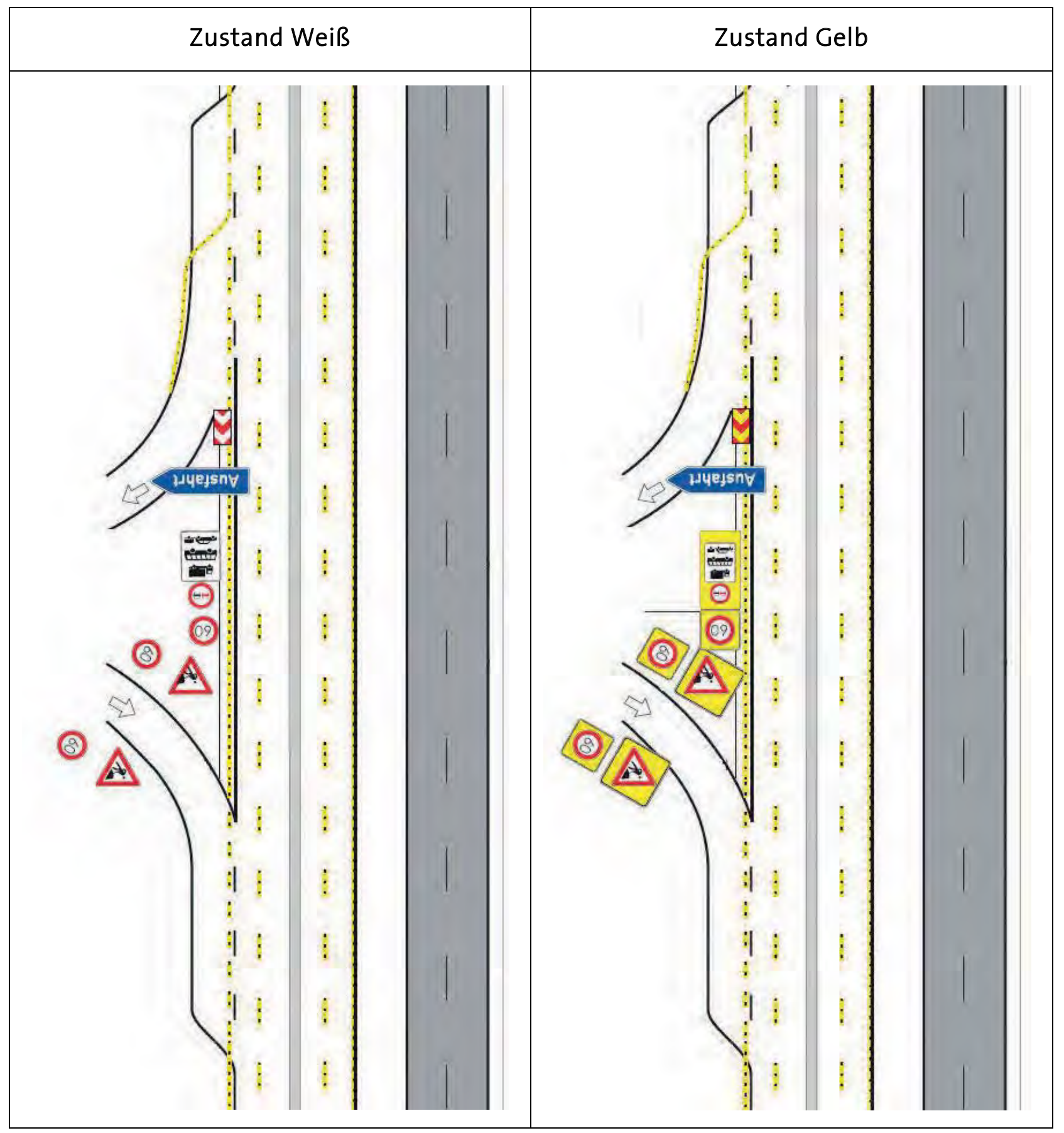

die dort aufgezeichneten Profile mit denen der verfolgten Fahrzeuge mit hoher Genauigkeit übereinstimmen.

Für die Baustelle konnten in zwei Zeiträumen ca. je 140 auswertbare Geschwindigkeitsprofile aufgenommen werden, die sich nach Richtung und Verkehrszustand wie in Tabelle 3-6 angegeben aufteilen. Somit stand zu jeder Variante ein Kollektiv von mindestens 30 Geschwindigkeitsprofilen für die Auswertung zur Verfügung. Durch die Unterscheidung zwischen verkehrsstarken und ver- 


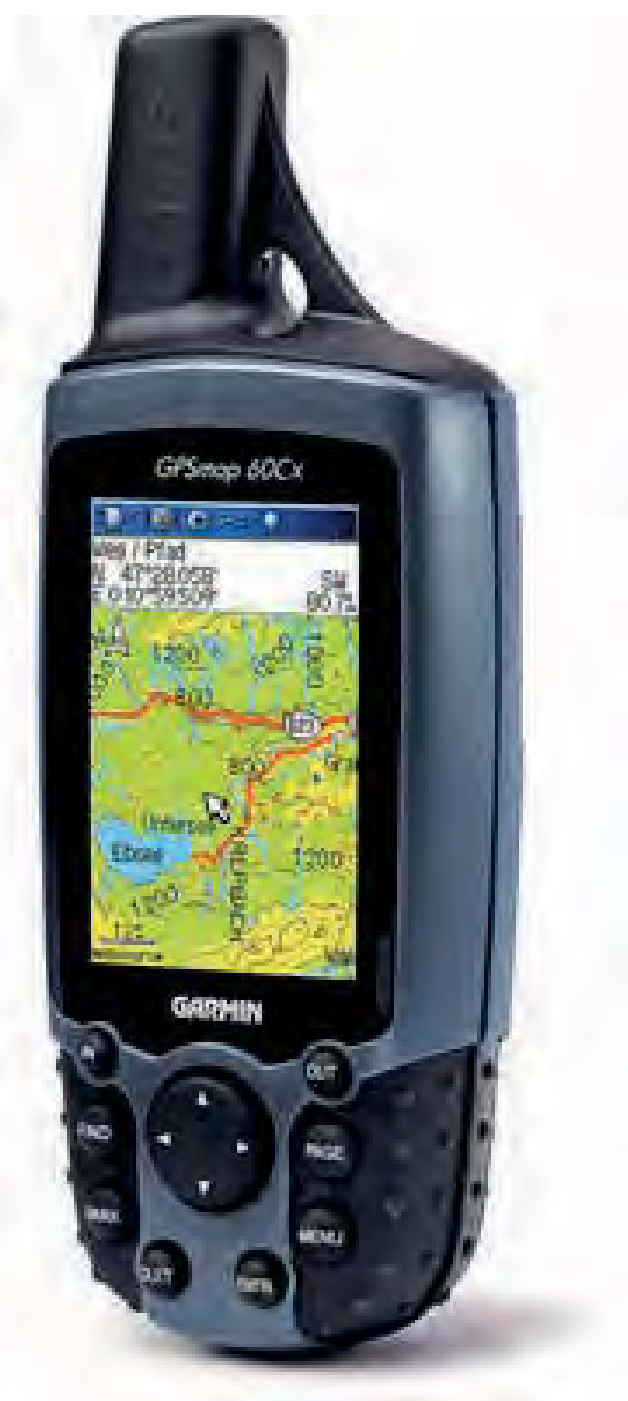

\begin{abstract}
Abbildung 3-6:
GARMIN GPSmap 60Cx (Quelle: http://www.navigationprofessionell.de/)
\end{abstract}

Tabelle 3-6:

Anzahl der ausgewerteten Geschwindigkeitsprofile kehrsschwachen Zeiten wurde angestrebt, sowohl typische durch das Verkehrsgeschehen beeinflusste Profile als auch die Geschwindigkeiten unbeeinflusst fahrender Fahrzeuge aufzuzeichnen. Neben der subjektiv betrachteten und bewerteten Verkehrssituation wurde die Reisegeschwindigkeit als objektive Größe zur Bestimmung des Verkehrszustandes hinzugezogen. Der Mittelwert über die Tagesganglinie zeigt die Differenzierung von verkehrsschwachen bzw. verkehrsstarken Zeiten. Diese wird beispielhaft in Abbildung 3-7 dargestellt:

Der Verlauf der Geschwindigkeitsprofile wurde für den gesamten Baustellenbereich und die in Kapitel 3.3 beschriebenen Bereiche ausgewertet.

\subsubsection{Querschnittsmessungen}

Die Querschnittsmessungen ergänzten die Erkenntnisse aus den Geschwindigkeitsprofilen auf der Grundlage eines größeren Fahrzeugkollektivs. Darüber hinaus sollten sie zusätzliche Informationen zum Abstandsverhalten und der Fahrstreifenwahl liefern. Dafür war die Anordnung der Messtechnik an zuvor für beide Untersuchungsphasen festgelegten und damit vergleichbaren Querschnitten wichtig. Die Erhebungen erfolgten mit Videotechnik. Die Kameras wurden auf Stativen mit einer Höhe von bis zu $5 \mathrm{~m}$ befestigt und nahe der Fahrbahn so aufgestellt, dass am jeweiligen Querschnitt alle Fahrstreifen der betrachteten Fahrtrichtung ausgewertet werden konnten. Dieses Verfahren bietet den Vorteil des mehrmaligen Auswertens sowie der Auswertung verschiedener Kenngrößen:

- Geschwindigkeiten

- Längsabstände

- Fahrzeugarten

- Fahrstreifenbelegung.

\begin{tabular}{|l|l|c|c|}
\hline Phase & Verkehrszustand & Fahrtrichtung 1 & Fahrtrichtung 2 \\
\hline \multirow{3}{*}{ weiß } & verkehrsschwach & 34 & 31 \\
\cline { 2 - 4 } & verkehrsstark & 37 & 39 \\
\hline \multirow{2}{*}{ gelb } & verkehrsschwach & 34 & 33 \\
\hline & verkehrsstark & 38 & 41 \\
\hline
\end{tabular}




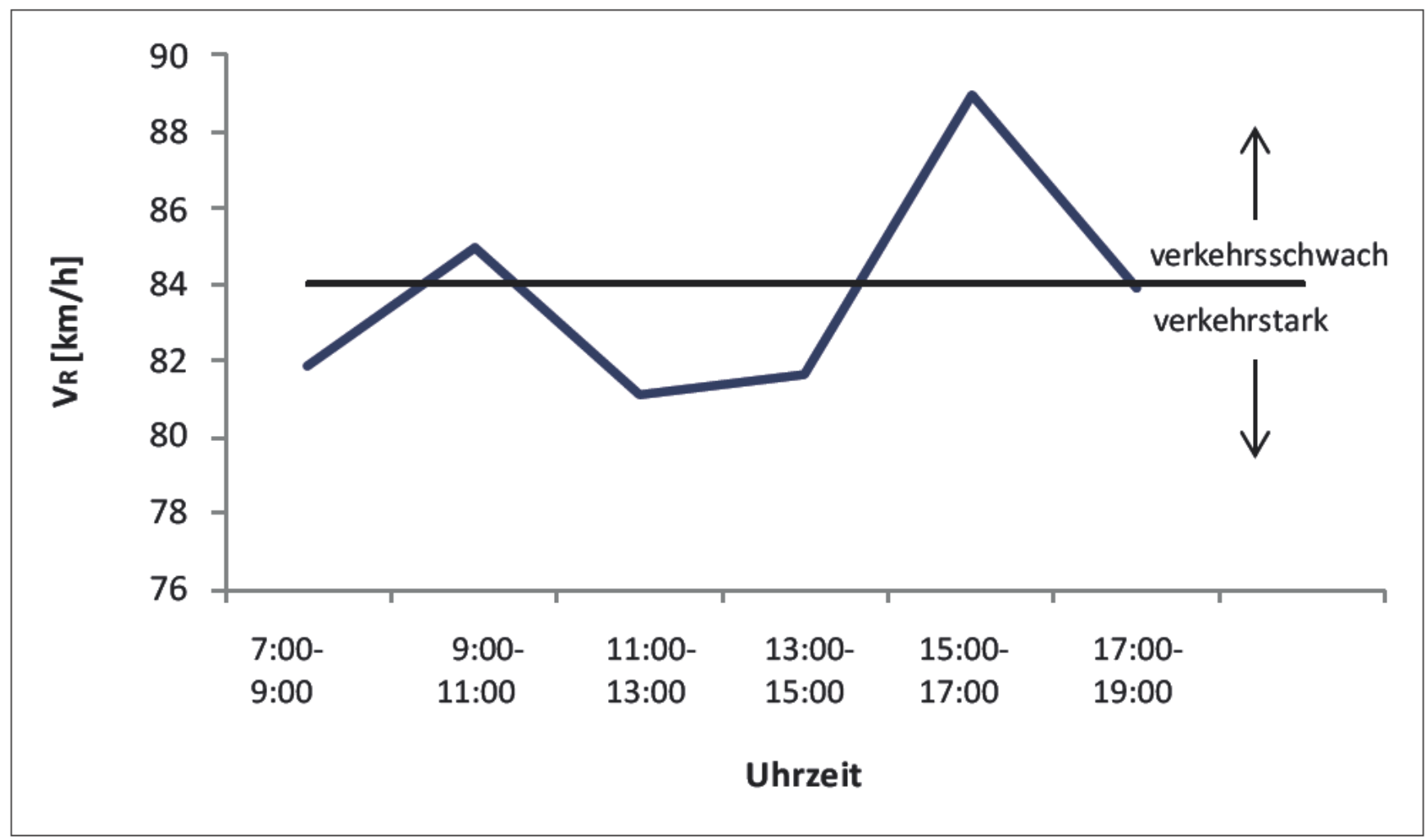

Abbildung 3-7:

Tagesganglinie der Fahrtrichtung 2 im Vorherzeitraum

Der Nachteil hierbei besteht darin, dass nicht immer Verdeckungen von Fahrzeugen auf einzelnen Fahrstreifen durch Fahrzeuge des Schwerverkehrs ausgeschlossen werden konnten.

Insgesamt wurden in jedem der beiden Zeiträume Messungen an 17 Querschnitten über jeweils mindestens drei Stunden durchgeführt, woraus sich insgesamt 102 Stunden Videoaufzeichnungen ergaben. Die Auswertung erfolgte manuell mit Hilfe eines Programms, welches über eine Einzelbildanzeige und eine Zeitangabe im 0,04-Sekundenbereich verfügte. An zwei festgelegten Querschnitten wurden die Zeiten der Überquerung der Fahrzeuge bestimmt. Zur Kontrolle der Plausibilität der Ergebnisse wurden dabei sowohl die Zeitpunkte der Überfahrten von Front und Heck des Fahrzeugs in einer Excel-Tabelle festgehalten. Zusätzlich erfolgte die Bestimmung der Fahrzeugart, differenziert nach Pkw, Transporter, Lkw und Sonstige.
Die Videoaufzeichnungen der einzelnen Querschnitte wurden jeweils über mindestens 30 Minuten ausgewertet. Konnte in diesem Zeitraum nicht die angestrebte Anzahl von 80 frei fahrenden Fahrzeugen erreicht werden, wurden die Auswertungen entsprechend ergänzt. Dabei wurde zunächst der Einfluss vorausfahrender Fahrzeuge auf die Geschwindigkeit des betrachteten Fahrzeugs in Abhängigkeit der Nettozeitlücke untersucht. Die Untersuchung und deren Ergebnisse sind beispielhaft für einen Querschnitt in Anhang D enthalten. Es wurde überprüft, ob sich in Abhängigkeit von den Nettozeitlücken zum vorausfahrenden Fahrzeug zwischen 5 s und 10 s ein signifikanter Unterschied bei den Geschwindigkeiten nachweisen lässt. Grundlage dieser Überprüfung war der statistische Vergleich der mittleren Geschwindigkeiten von Fahrzeugkollektiven mit unterschiedlichen Längsabständen (Anhang D). Mit Ausnahme einer Konstellation konnten keine Unterschiede zwischen den Geschwindigkeiten der Fahrzeuge mit mindestens 5 s und bis hin zur 
Gruppe der Fahrzeuge mit mindestens 10 s Abstand zum Vordermann nachgewiesen werden.

Gleichzeitig konnte aber auch die Beeinflussung der Geschwindigkeiten durch (dicht auffahrende) Folgefahrzeuge nicht ausgeschlossen werden. Aus diesem Grund wurden auch diese Abstände überprüft. Für die Abstände zu den folgenden Fahrzeugen, die im Bereich von weniger als $1 \mathrm{~s}$ bis $5 \mathrm{~s}$ betrachtet wurden, ließ sich nur in Einzelfällen ein Zusammenhang nachweisen. Allerdings zeigten Fahrzeuge, deren Folgefahrzeug weniger als 1 s Abstand hielt eine Tendenz zu geringeren Geschwindigkeiten auf gegenüber den Fahrzeugen, welchen mit größerem Abstand gefolgt wurde. Aufbauend auf diesen Erkenntnissen galten für die weiteren Auswertungen solche Fahrzeuge als frei fahrend, die mindestens einen Abstand von mindestens $5 \mathrm{~s}$ zum vorausfahrenden und mehr als $1 \mathrm{~s}$ zum folgenden Fahrzeug aufwiesen. Diese für Autobahnen vergleichsweise kurzen Abstände sind auch vor dem Hintergrund des an den meisten Messquerschnitten aufgetretenen geringen Geschwindigkeitsniveaus und der besonderen Verkehrssituation zu interpretieren.

\section{Annäherungsbereiche}

Die Anordnung der Querschnitte für die lokalen Messungen am Beginn der Baustelle ist beispielhaft in Abbildung 38 dargestellt. Die Messquerschnitte wurden dabei so gewählt, dass die Wirkung von Beschränkungen der zulässigen Höchstgeschwindigkeiten und die Geschwindigkeiten bei der Einfahrt in die Verschwenkung bzw. Überleitung der Fahrstreifen bestimmt werden konnten.

In der abgeschlossenen Untersuchung (OQSV, 2010) wurde starkes Abbremsen kurz vor Baustellenbeginn und damit häufiger auftretende Auffahrunfälle festgestellt. Der Querschnitt 2 dient in erster Linie der Bestimmung des Bereichs der maßgebenden Geschwindigkeitsreduzierung bei der Annäherung an die Baustelle. Es soll so überprüft werden, ob sich durch die Hervorhebung der relevanten Beschilderung eine frühzeitigere und homogenere Geschwindigkeitsreduzierung erreichen lässt.

Insgesamt konnten unter den beschriebenen Randbedingungen für frei fahrende Fahrzeuge auf dem rech-

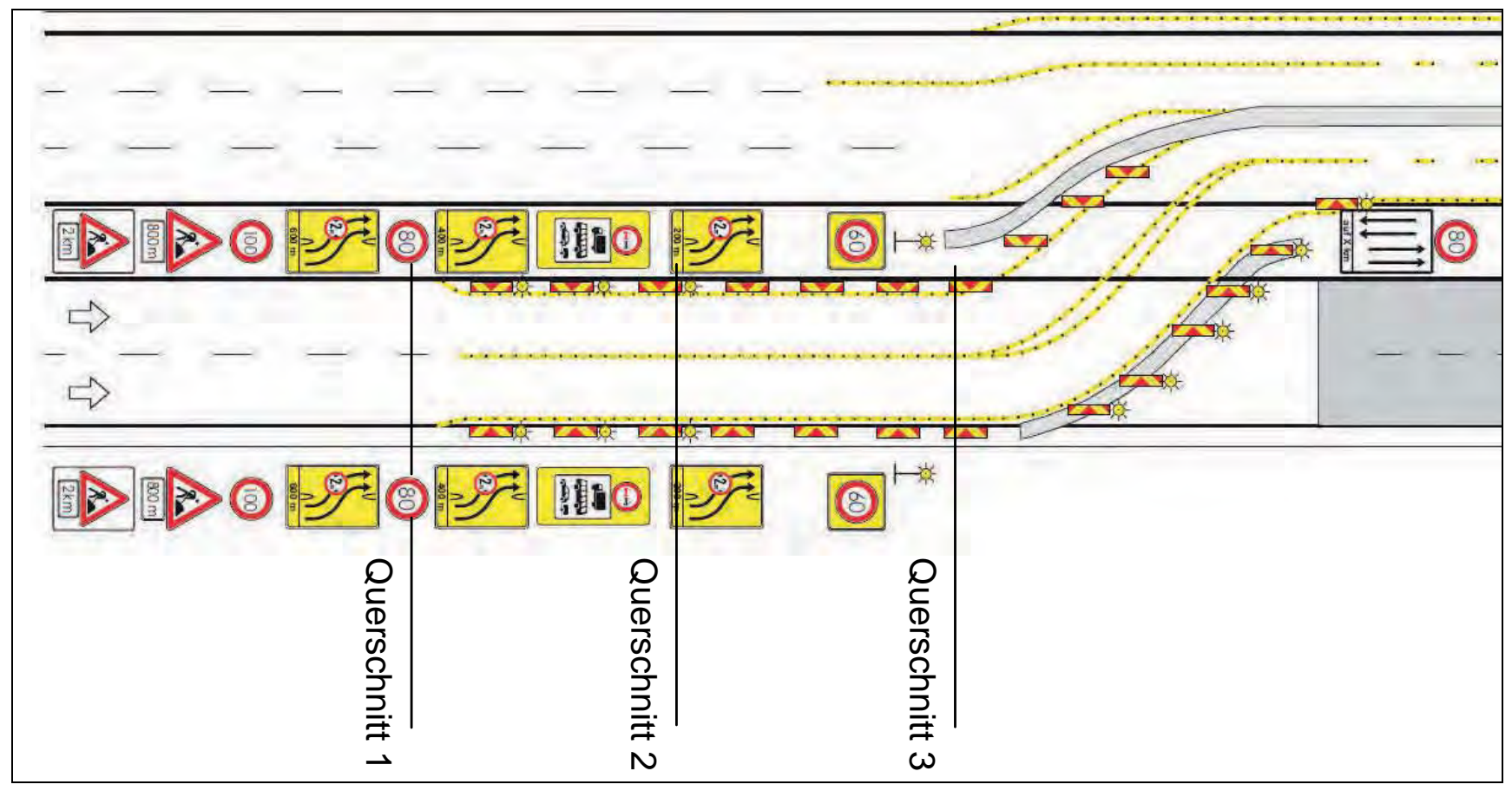

Abbildung 3-8:

Messquerschnitte im Annäherungsbereich der Fahrtrichtung 1 
ten Fahrstreifen jeweils etwa $28 \%$ der gemessenen Fahrzeuge als frei in ihrer Geschwindigkeitswahl gelten. Auf dem linken Fahrstreifen ist dieser Anteil größeren Schwankungen unterlegen (Tabelle 3-7 und Tabelle 3-8).

\section{Baustellenende}

Zur Überprüfung der Wirkung der Beschilderung auf das

Fahrverhalten wurden am Baustellenende die Ergebnisse

\section{Tabelle 3-7}

Querschnittsmessungen im Annäherungsbereich der Fahrtrichtung 1 - gemessene Fahrzeuge

\begin{tabular}{|c|c|c|c|c|c|c|}
\hline \multirow[t]{2}{*}{ Phase } & \multirow[t]{2}{*}{ Querschnitt } & \multirow{2}{*}{$\begin{array}{l}\text { Abstand } \\
\text { bis } \\
\text { Überleitung }\end{array}$} & \multicolumn{2}{|c|}{ Fahrstreifen 1 (rechts) } & \multicolumn{2}{|c|}{ Fahrstreifen 2 (links) } \\
\hline & & & $\begin{array}{c}\text { gesamt } \\
{[\mathrm{Fz}]}\end{array}$ & $\begin{array}{c}\text { frei } \\
\text { fahrend } \\
{[F z]}\end{array}$ & $\begin{array}{c}\text { gesamt } \\
{[\mathrm{Fz}]}\end{array}$ & $\begin{array}{c}\text { frei } \\
\text { fahrend } \\
{[\mathrm{Fz}]}\end{array}$ \\
\hline \multirow{3}{*}{ Weiß } & 1 & $500 \mathrm{~m}$ & 406 & 97 & 184 & 83 \\
\hline & 2 & $200 \mathrm{~m}$ & 599 & 139 & 211 & 84 \\
\hline & 3 & $0 \mathrm{~m}$ & 609 & 135 & 220 & 66 \\
\hline \multirow{3}{*}{ Gelb } & 1 & $500 \mathrm{~m}$ & 384 & 107 & 186 & 58 \\
\hline & 2 & $200 \mathrm{~m}$ & 578 & 151 & 226 & 58 \\
\hline & 3 & $0 \mathrm{~m}$ & 592 & 135 & 241 & 58 \\
\hline
\end{tabular}

Tabelle 3-8:

Querschnittsmessungen im Annäherungsbereich der Fahrtrichtung 2 - gemessene Fahrzeuge

\begin{tabular}{|c|c|c|c|c|c|c|}
\hline \multirow[t]{2}{*}{ Phase } & \multirow[t]{2}{*}{ Querschnitt } & \multirow{2}{*}{$\begin{array}{c}\text { Abstand } \\
\text { bis } \\
\text { Überleitung }\end{array}$} & \multicolumn{2}{|c|}{$\begin{array}{c}\text { Fahrstreifen } 1 \\
\text { (rechts) }\end{array}$} & \multicolumn{2}{|c|}{$\begin{array}{l}\text { Fahrstreifen } 2 \\
\text { (links) }\end{array}$} \\
\hline & & & $\begin{array}{c}\text { gesamt } \\
{[\mathrm{Fz}]}\end{array}$ & $\begin{array}{l}\text { frei fahrend } \\
\qquad[\mathrm{Fz}]\end{array}$ & $\begin{array}{c}\text { gesamt } \\
{[\mathrm{Fz}]}\end{array}$ & $\begin{array}{l}\text { frei fahrend } \\
\qquad[\mathrm{Fz}]\end{array}$ \\
\hline \multirow{4}{*}{ Weiß } & 1 & $600 \mathrm{~m}$ & 309 & 120 & 190 & 93 \\
\hline & 2 & $500 \mathrm{~m}$ & 359 & 99 & 182 & 82 \\
\hline & 3 & $200 \mathrm{~m}$ & 373 & 116 & 176 & 79 \\
\hline & 4 & $0 \mathrm{~m}$ & 451 & 89 & 244 & 72 \\
\hline \multirow{4}{*}{ Gelb } & 1 & $600 \mathrm{~m}$ & 303 & 124 & 215 & 68 \\
\hline & 2 & $500 \mathrm{~m}$ & 400 & 112 & 172 & 83 \\
\hline & 3 & $200 \mathrm{~m}$ & 398 & 101 & 181 & 89 \\
\hline & 4 & $0 \mathrm{~m}$ & 382 & 72 & 177 & 82 \\
\hline
\end{tabular}


der Nachfolgefahrten durch die von vier Querschnittsmessungen je Betrachtungsphase ergänzt. Auf diese Weise wurde überprüft, ob sich im Vergleich zu den bisherigen Untersuchungen (QSV, 2010) eine moderatere Beschleunigung bei den gemessenen Fahrzeugen einstellt und ob diese tatsächlich erst nach dem Ende der Verschwenkung bzw. Überleitung der Fahrstreifen eintritt.

In Fahrtrichtung 1 wurden an jedem Querschnitt zwischen 420 und 610 Fahrzeugen erfasst (Tabelle 3 9). Im Verlauf des betrachteten Abschnitts steigt der Anteil der linksfahrenden Fahrzeuge von ca. $13 \%$ auf über $30 \%$ der Querschnittsverkehrsstärke an. Aus diesem Grund konnten an Querschnitt 1 auf dem linken Fahrstreifen nur sehr wenig frei fahrende Fahrzeuge (im Vergleich zu anderen Messstellen bei vergleichbaren Querschnittsverkehrsstärken) erfasst werden.

Analog zur Fahrtrichtung 1 nimmt auch in der Gegenrichtung am Ende der Baustelle der Anteil der Linksfahrer kontinuierlich zu. Hier liegt das Ausgangsniveau aber bei über $18 \%$ an Querschnitt 1 und erreicht an Querschnitt 4 bis zu 40 \% (Phase Gelb) (Tabelle 3-10).

\subsection{Unfallanalyse}

Der mit vier Monaten für eine Unfallanalyse zu kurze Betrachtungszeitraum lässt keine stabilen Aussagen zur Entwicklung des Unfallgeschehens zu. Ein Vergleich von Unfallkenngrößen kann in diesem Zusammenhang aufgrund der geringen Anzahl und Dauer der Betrachtung nicht erfolgen. Die Ergebnisse würden auf Einzelereignissen beruhen und großen Streuungen unterliegen.

Stattdessen wurden die Beschreibung des Unfallhergangs und -ortes von den zuständigen Polizeidienststellen zur Verfügung gestellt und ausgewertet. Dabei sollten die Erkenntnisse zum Unfallgeschehen vor dem Hintergrund der veränderten Beschilderung betrachtet werden.

\subsection{Gesamtanalyse}

Die Ergebnisse der Querschnittsmessungen sowie der Nachfolgefahrten wurden zunächst für den Vorher- / Nachher-Zeitraum gegenübergestellt. So konnten Aussagen zur Wirkung der gelben Beschilderung von unfal-

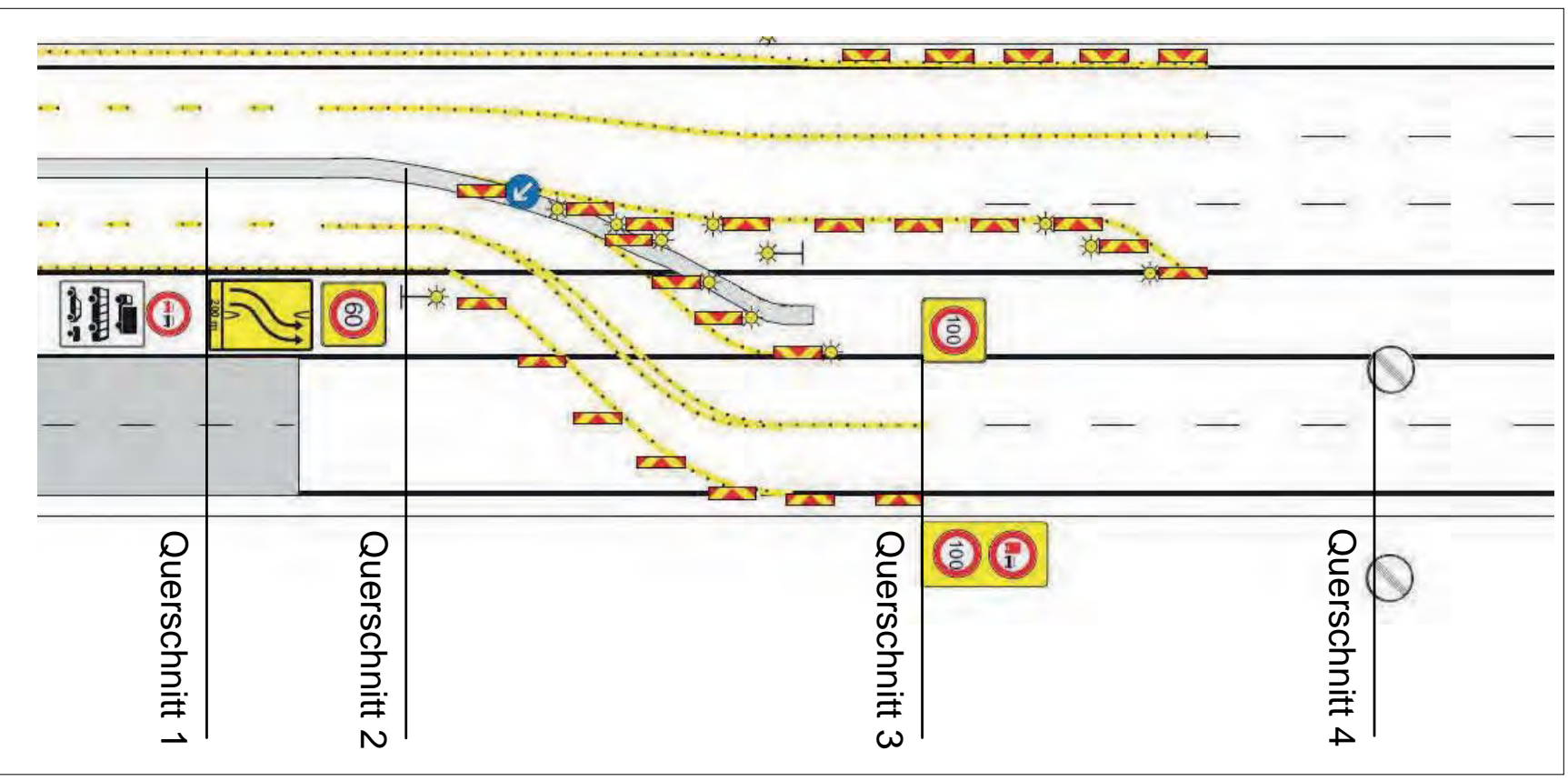

Abbildung 3-9:

Messquerschnitte am Baustellenende der Fahrtrichtung 1 
Tabelle 3-9:

Querschnittsmessungen am Baustellenende der Fahrtrichtung 1 - gemessene Fahrzeuge

\begin{tabular}{|c|c|c|c|c|c|c|}
\hline \multirow[t]{2}{*}{ Phase } & \multirow[t]{2}{*}{ Querschnitt } & \multirow{2}{*}{$\begin{array}{l}\text { Abstand } \\
\text { bis } \\
\text { Überleitung }\end{array}$} & \multicolumn{2}{|c|}{ Fahrstreifen 1 (rechts) } & \multicolumn{2}{|c|}{ Fahrstreifen 2 (links) } \\
\hline & & & $\begin{array}{c}\text { gesamt } \\
{[\mathrm{Fz}]}\end{array}$ & $\begin{array}{c}\text { frei } \\
\text { fahrend } \\
{[\mathrm{Fz}]}\end{array}$ & $\begin{array}{c}\text { gesamt } \\
\text { [Fz] }\end{array}$ & $\begin{array}{c}\text { frei } \\
\text { fahrend } \\
{[\mathrm{Fz}]}\end{array}$ \\
\hline \multirow{4}{*}{ Weiß } & 1 & $200 \mathrm{~m}$ & 382 & 81 & 145 & 81 \\
\hline & 2 & $0 \mathrm{~m}$ & 450 & 80 & 181 & 56 \\
\hline & 3 & $-20 m$ & 421 & 82 & 199 & 81 \\
\hline & 4 & $-500 \mathrm{~m}$ & 299 & 99 & 175 & 78 \\
\hline \multirow{4}{*}{ Gelb } & 1 & $200 \mathrm{~m}$ & 401 & 83 & 157 & 81 \\
\hline & 2 & $0 \mathrm{~m}$ & 396 & 84 & 152 & 59 \\
\hline & 3 & $-20 m$ & 340 & 87 & 134 & 80 \\
\hline & 4 & $-500 m$ & 310 & 102 & 195 & 78 \\
\hline
\end{tabular}

Tabelle 3-10:

Querschnittsmessungen am Baustellenende der Fahrtrichtung 2-gemessene Fahrzeuge

\begin{tabular}{|c|c|c|c|c|c|c|}
\hline \multirow{2}{*}{ Phase } & \multirow[t]{2}{*}{ Querschnitt } & \multirow{2}{*}{$\begin{array}{l}\text { Abstand } \\
\text { bis } \\
\text { Überleitung }\end{array}$} & \multicolumn{2}{|c|}{ Fahrstreifen 1 (rechts) } & \multicolumn{2}{|c|}{ Fahrstreifen 2 (links) } \\
\hline & & & $\begin{array}{c}\text { gesamt } \\
{[\mathrm{Fz}]}\end{array}$ & $\begin{array}{c}\text { frei } \\
\text { fahrend } \\
{[\mathrm{Fz}]}\end{array}$ & $\begin{array}{c}\text { gesamt } \\
{[\mathrm{Fz}]}\end{array}$ & $\begin{array}{c}\text { frei } \\
\text { fahrend } \\
{[F z]}\end{array}$ \\
\hline \multirow{4}{*}{ Weiß } & 1 & $200 \mathrm{~m}$ & 402 & 78 & 158 & 79 \\
\hline & 2 & $0 \mathrm{~m}$ & 404 & 89 & 191 & 84 \\
\hline & 4 & $-20 \mathrm{~m}$ & 408 & 90 & 166 & 80 \\
\hline & 4 & $-500 \mathrm{~m}$ & 327 & 99 & 182 & 66 \\
\hline \multirow{4}{*}{ Gelb } & 1 & $200 \mathrm{~m}$ & 432 & 75 & 161 & 74 \\
\hline & 2 & $0 \mathrm{~m}$ & 483 & 150 & 157 & 73 \\
\hline & 3 & $-20 m$ & 429 & 68 & 155 & 76 \\
\hline & 4 & $-500 \mathrm{~m}$ & 292 & 101 & 195 & 75 \\
\hline
\end{tabular}


lauffälligen Bereichen auf das Fahrverhalten abgeleitet werden. Im Anschluss wurden die gewonnenen Erkenntnisse aus der Untersuchung des Verkehrsablaufs und des Unfallgeschehens zusammengeführt.

Dabei wurden alle Erkenntnisse dieser Untersuchungen unter Berücksichtigung der Ergebnisse aus (QSV, 2010) interpretiert. Dabei ließen sich Zusammenhänge zwischen dem Verkehrsablauf und dem Unfallgeschehen herausarbeiten und auf die möglichen Auswirkungen gelber Beschilderungen übertragen. Auf diesem Weg lässt sich der grundsätzliche Einfluss der gelben Beschilderung auf die Verkehrssicherheit ableiten. Ergänzend werden die Aussagen der Literatur und früherer Untersuchungen (BAIER et al., 2006) mit den Erkenntnissen abgeglichen.

Abschließend wurden Empfehlungen für den Einsatz von gelber Beschilderung von unfallauffälligen Abschnitten in Baustellen auf Autobahnen formuliert. Dabei stehen Anordnung und Einsatzgrenzen von aufmerksamkeitserhöhenden Maßnahmen im Vordergrund.

\section{Analyse des Verkehrsablaufs}

\subsection{Allgemeines}

Im Vergleich zu den Baustellen der Untersuchung von QSV (2010) wies die hier untersuchte Baustelle eher hohe Geschwindigkeiten auf. Sie sind vergleichbar mit der Baustelle D1 (bei Aachen). Zum Verlauf der Geschwindigkeiten konnten mit anderen Baustellen vergleichbare Ergebnisse beobachtet werden. Die mittleren Geschwindigkeiten zeigen im Baustelleninnenbereich über die Länge einen gleichmäßigen Verlauf. Es wurden in Einzelfällen Geschwindigkeiten bis zu 120 km/h gemessen. Die größten Geschwindigkeitsüberschreitungen wurden, wie auch in den Baustellen der abgeschlossenen Untersuchung, in den Bereichen mit vzul = $60 \mathrm{~km} / \mathrm{h}$ festgestellt.

Für die Untersuchung der Auswirkung selektivgelber Beschilderung auf das Fahrverhalten wurden neben den Geschwindigkeiten auch die Anteile der Fahrstreifenbelegungen und der Längsabstände zwischen den Fahrzeugen betrachtet. Dabei konnten an einzelnen Messquerschnitten im Zeitraum der gelben Beschilderung geringere Anteilswerte bei der Unterschreitung des Sicherheitsabstands festgestellt werden. Dies betraf vorrangig die Abstände auf dem Überholfahrstreifen im Bereich der Anschlussstelle und am Baustellenende im Bereich Zeichens 274-60 ( $\left.\mathrm{z}_{\mathrm{zul}}=100 \mathrm{~km} / \mathrm{h}\right)$. Allerdings konnte am Beginn der Baustelle, wo der Fahrer zuerst mit der gelben Beschilderung konfrontiert wird, keine Veränderung des Abstandsverhaltens erkannt werden ( $v g$. Anhang E).

Die Anteilswerte der Fahrzeuge auf dem Überholfahrstreifen (Anhang F), aber insbesondere aus den Geschwindigkeiten lässt sich ein Einfluss der gelben Beschilderung bei geringer Verkehrsstärke ableiten. So ließ sich insbesondere am Baustellenbeginn vor der Fahrstreifenverschwenkung durch eine gelbe Beschilderung eine Reduzierung der Geschwindigkeiten erreichen. Die Ergebnisse sind bereichsbezogen in den folgenden Kapiteln dargestellt. 


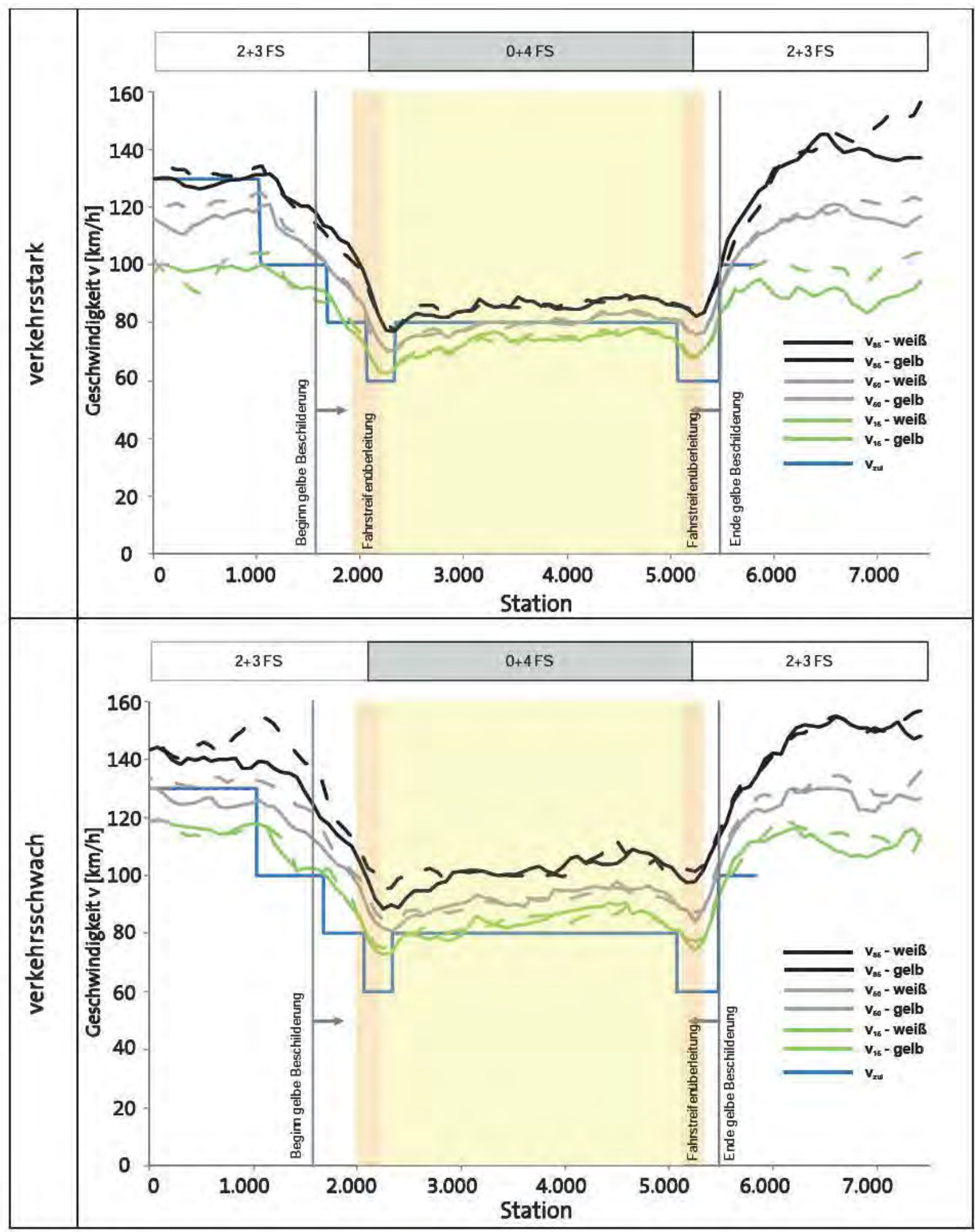

Abbildung 4-1:

Geschwindigkeitsverlauf über den untersuchten Bereich zu Zeiten hoher und geringer Verkehrsstärken 
Die Geschwindigkeitsprofile wurden differenziert nach verkehrsstarken und-schwachen Zeiten bestimmt (vgl. Kapitel 3.4.1). In den verkehrsschwachen Zeiten können Fahrer eher ihre Wunschgeschwindigkeit fahren, dagegen werden sie zu verkehrsstarken Zeiten häufiger durch andere Fahrzeuge in ihrem Verhalten beeinflusst. Daraus ergaben sich für die verkehrsschwachen und verkehrsstarken Zeiten unterschiedliche Ergebnisse. Während sich aus den Geschwindigkeitsprofilen der verkehrsstarken Zeiten keine wesentlichen Unterschiede zwischen den Zuständen „weiß" und "gelb" feststellen ließen (Abbildung 4-1), war bei geringeren Verkehrsstärken ein Einfluss der modifizierten Beschilderung zu erkennen. Dieser hängt jedoch von den betrachteten Bereichen ab. Eine vertiefte Betrachtung der untersuchten Bereichewird in den nachfolgenden Kapiteln vorgenommen. Generell ist der Einflussbereich der selektivgelben Beschilderung begrenzt. Im weiteren Verlauf der Baustelle nähern sich die Geschwindigkeiten vom „gelben“ Zustand dem „weißen“" Zustand wieder an.

Aus den Geschwindigkeitsprofilen der Nachfolgefahrten konnten außerdem Aussagen zur Homogenität der Geschwindigkeit im Baustelleninnenbereich getroffen werden. Dafür wurde die Differenz der $v_{85}$ und $v_{50}$ gebildet und über die Länge abgetragen. Es ist zu erkennen, dass insbesondere im Annäherungsbereich das Geschwindigkeitsverhalten bei gelber Beschilderung wesentlich homogener geworden ist. So beträgt beispielsweise die Differenz an der Station 1+950 (Abbildung 4-2) in der gelben Phase $v_{85}-v_{50}=8 \mathrm{~km} / \mathrm{h}$ und in der weißen Phase $v_{85}-v_{50}=22 \mathrm{~km} / \mathrm{h}$. Eine ähnliche Tendenz zeigt sich auch im Bereich der Anschlussstelle und bei der Ausfahrt.

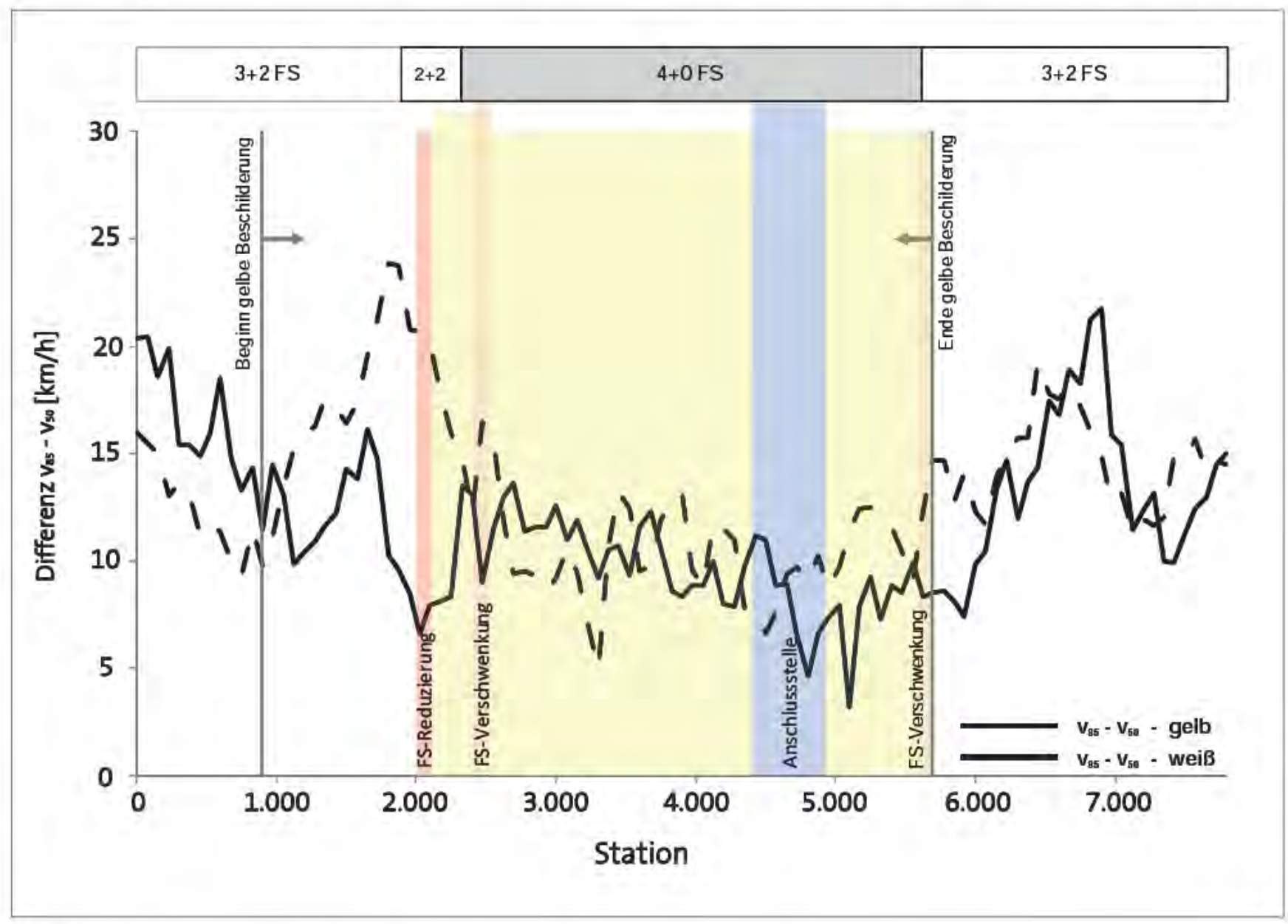

Abbildung 4-2:

Homogenität der Geschwindigkeiten - Differenz der Geschwindigkeitskenngrößen $\mathbf{v}_{85}-\mathbf{v}_{50}$ 


\subsection{Annäherungsbereich und erste Verschwenkung}

Die Annäherungsbereiche der beiden betrachteten Fahrtrichtungen unterschieden sich in ihrer Gestaltung (vgl. Kapitel 3.3.2). Da auch auf die Fahrstreifenreduzierung $500 \mathrm{~m}$ vor der Fahrstreifenverschwenkung am Baustellenbeginn mit gelben Einengungstafeln (Zeichen 522-11) hingewiesen wurde, begann in Fahrtrichtung 2 die gelbe Beschilderung $1.100 \mathrm{~m}$ vor der Baustelle. In Fahrtrichtung 1 waren dagegen nur die letzten $600 \mathrm{~m}$ vor der Überleitung der Fahrstreifen auf die Gegenfahrbahn mit gelben Überleitungstafeln (Zeichen 500) und Pfeilbaken ausgestattet.

\section{$\underline{\text { Geschwindigkeitsverläufe }}$}

Die beiden untersuchten Beschilderungszustände weiß und gelb wiesen am Beginn des Annäherungsbereichs ${ }^{2}$ vergleichbare Geschwindigkeiten auf. Das geht aus allen drei betrachteten Geschwindigkeitskenngrößen $v_{15}, v_{50}$ und $\mathrm{V}_{85}$, die beispielhaft für den Annäherungsbereich der Fahrtrichtung 1 in Abbildung 4-4 dargestellt sind, hervor. Bereits auf Höhe der ersten Überleitungs-/Fahrstreifentafeln gingen die Geschwindigkeiten im Zustand Gelb zurück. Mit $7 \mathrm{~km} / \mathrm{h}$ wies die $\mathrm{v}_{85}$ den größten Rückgang auf. Bezogen auf die Ausgangsgeschwindigkeit am Zeichen 123 ergibt sich daraus gegenüber dem Zustand Weiß ein um 4 Prozent

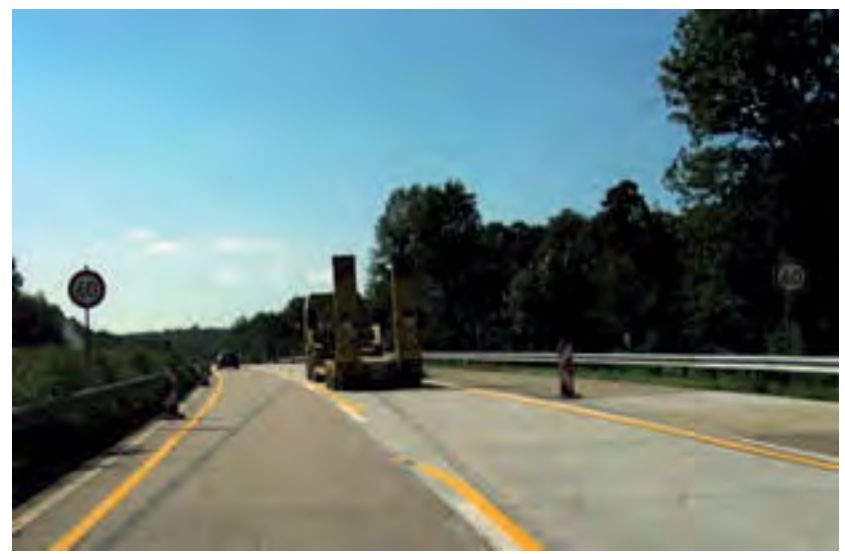

größerer Geschwindigkeitsrückgang an der ersten Fahrstreifenverschwenkung (Abbildung 4-5). Die Verringerung der anderen Geschwindigkeitskenngrößen war in dieser Fahrtrichtung weniger deutlich und betrug lediglich $3 \%$ der Geschwindigkeit im Zustand Weiß (Tabelle 4-1).

In Fahrtrichtung 2 ist der Geschwindigkeitsrückgang am ersten Messquerschnitt gegenüber der Ausgangsgeschwindigkeit sogar um sieben Prozent größer (Abbildung 4-6). Allerdings lag die $v_{85}$ der Ausgangsgeschwindigkeit im gelben Zustand etwa $5 \mathrm{~km} / \mathrm{h}$ über dem des weißen Zustands, so dass der höhere anteilige Geschwindigkeitsrückgang ebenfalls eine Differenz von $7 \mathrm{~km} / \mathrm{h}$ am ersten Messquerschnitt beschreibt. Die Verringerung insbesondere der $\mathrm{v}_{85}$ im Zustand Gelb weist auf eine geringere Bandbreite der Geschwindigkeiten zwischen den Fahrzeugen und damit eine Homogenisierung der Geschwindigkeiten hin.

Der Anstieg der v $_{85} \mathrm{im}$, weißen“ Zustand auf $154 \mathrm{~km} / \mathrm{h} \mathrm{im}$ Bereich der Verkehrsbeeinflussungsanlage (VBA, Station $1+030)$ ist auf zufällige Einzelereignisse zurückzuführen (siehe Abbildung 1). In der gelben Phase steigt die $v_{85}$ in diesem Abschnitt nicht an. Da von dieser Station aus jedoch die erste Verschwenkungstafel (Z 505-12-600) noch nicht zu sehen ist, kann der Unterschied von „weiß" zu "gelb“ nicht der modifizierten Beschilderung zugerechnet werden.

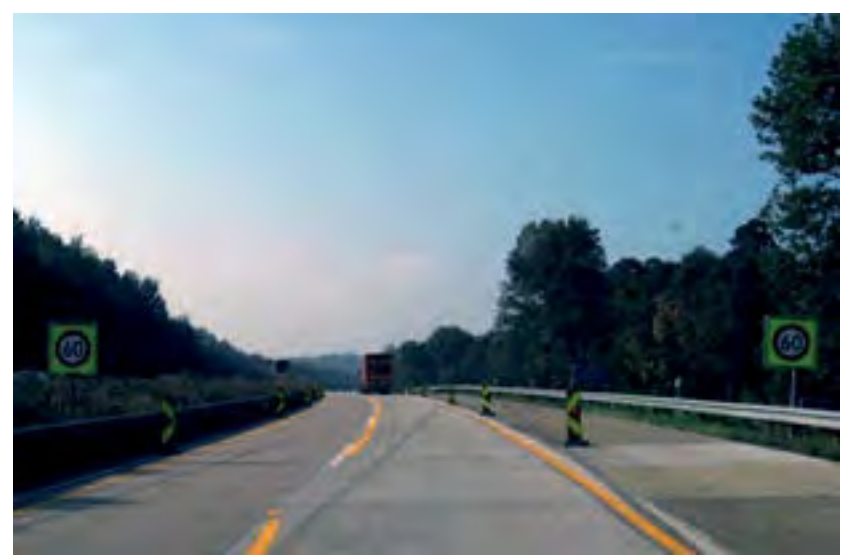

Abbildung 4-3:

Fahrtrichtung 1 Querschnitt 100 m vor der Fahrstreifenüberleitung nach RSA 95 (links) und mit modifizierter Beschilderung (rechts) 


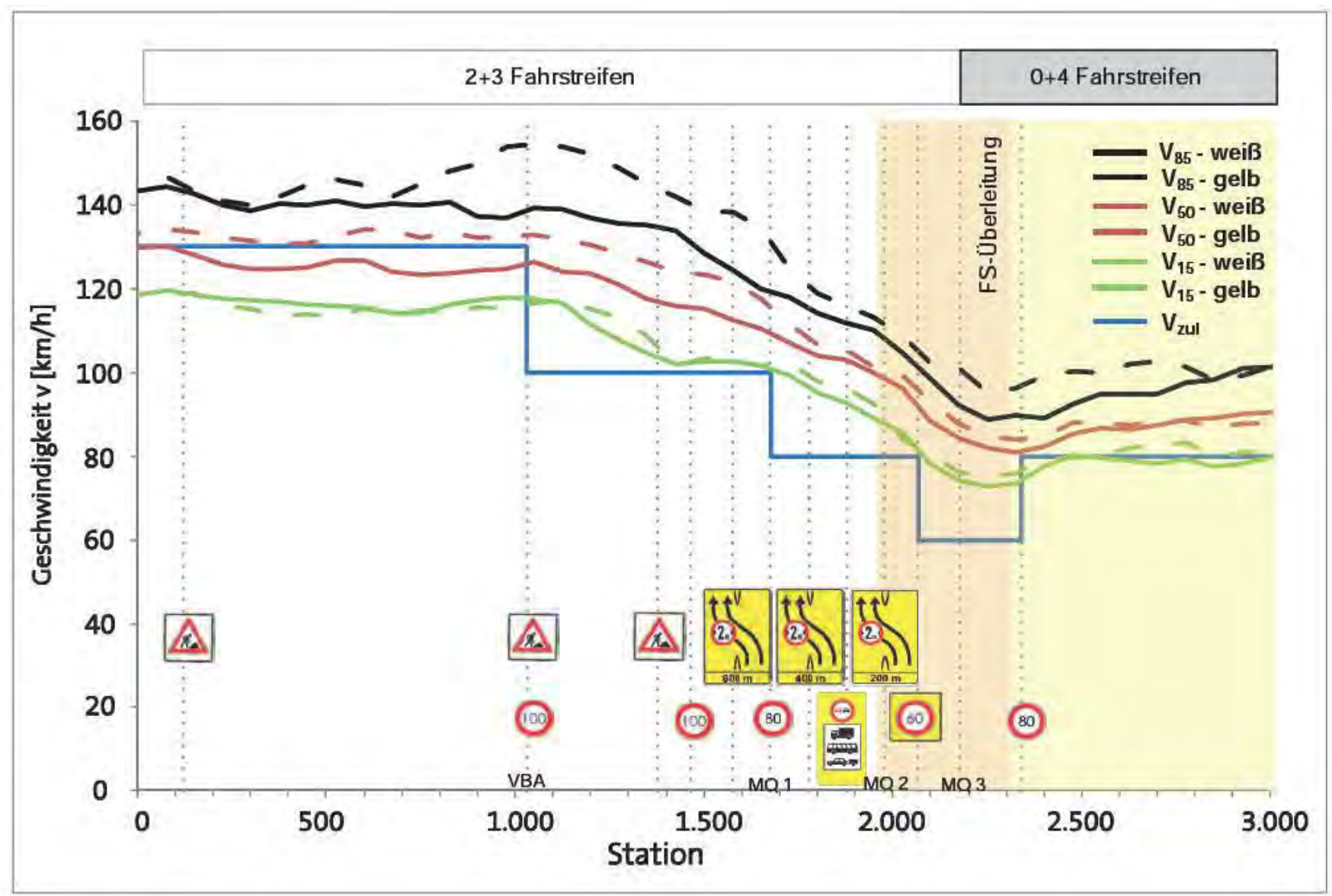

Abbildung 4-4:

Verläufe der Geschwindigkeitskenngrößen v15, v50 und v85 im Annäherungsbereich der Fahrtrichtung 1 nach Beschilderungsart bei geringen Verkehrsstärken

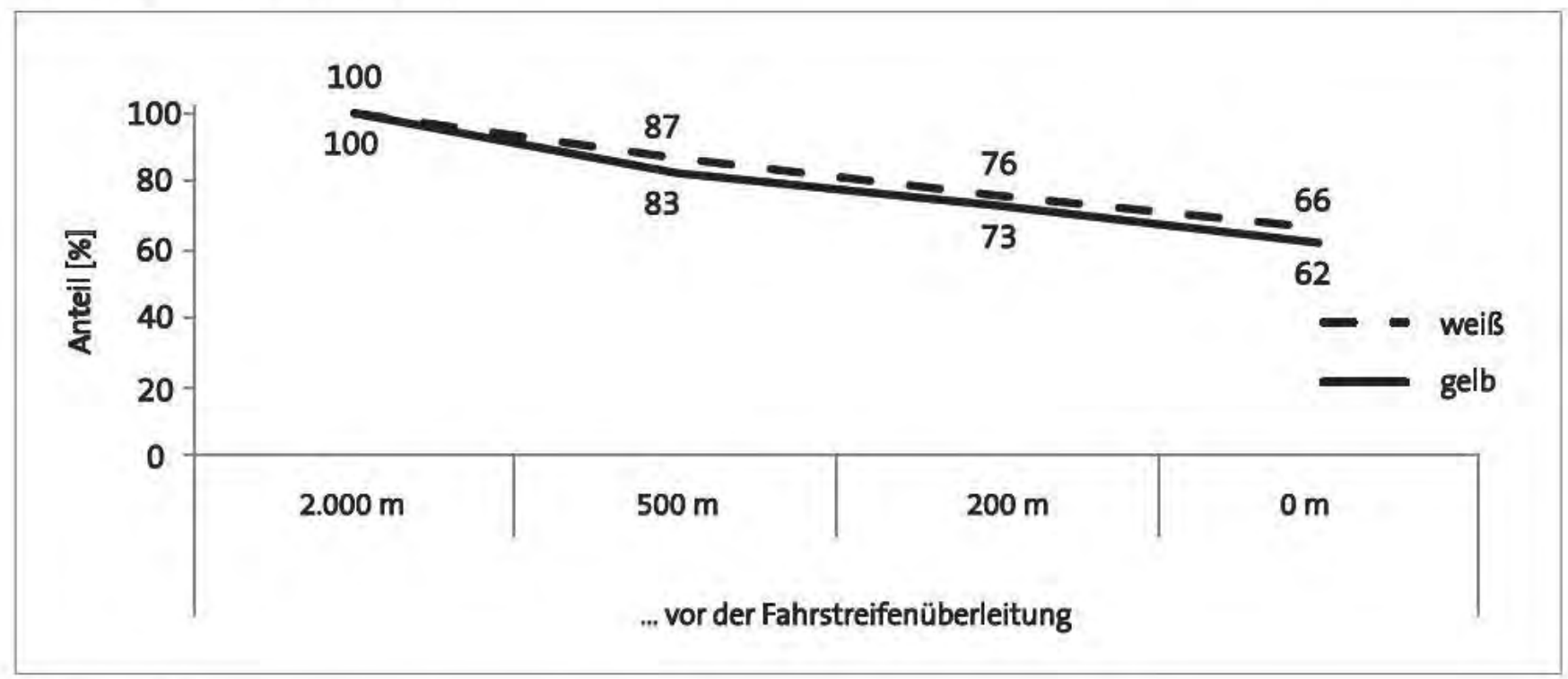

Abbildung 4-5:

Anteil des Rückgangs der v85 im Annäherungsbereich der Fahrtrichtung 1, verkehrsschwach 


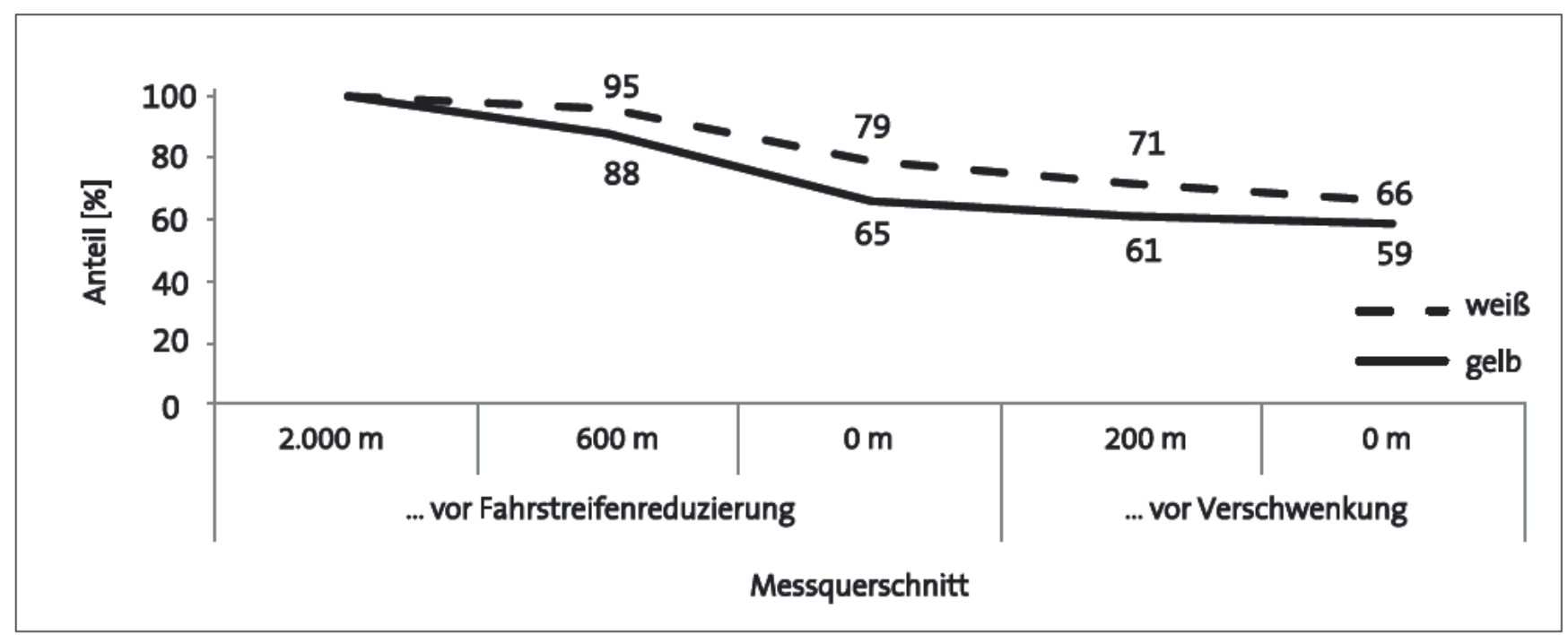

Abbildung 4-6:

Anteil des Rückgangs der v85 im Annäherungsbereich der Fahrtrichtung 2, verkehrsschwach

Die Differenzen der $\mathrm{v}_{85}-$ und $\mathrm{v}_{15}$, welche die Bandbreite der gefahrenen Geschwindigkeiten darstellen, sind in den Annäherungsbereichen gegenüber dem weißen Beschilderungszustand bei gelb geringer. Demnach führte die gelbe Beschilderung zu einem Rückgang der Geschwindigkeiten insbesondere bei den schneller fahrenden Verkehrsteilnehmern. Dies erfolgte bereits zu Beginn der gelben Beschilderung. Im weiteren Verlauf der Annäherungsbereiche nahmen die Geschwindigkeiten bei weißer und gelber Beschilderung in vergleichbarem Maß ab.

Im Verlauf der Annäherungsbereiche verringerte sich die $v_{85}$ zwischen den ersten Zeichen 123 (Baustelle) und dem Beginn der Fahrstreifenverschwenkung im weißen Zustand in jeder Fahrtrichtung um $34 \%$. Der Rückgang der anderen zwei Geschwindigkeitskenngrößen betrug zwischen $36 \%$ und $38 \%$ und war demnach etwas stärker ausgeprägt. Schnelle Fahrer verringerten bei weißer Beschilderung ihre Geschwindigkeiten im Annäherungsbereich weniger stark als langsamere. Dennoch wurden die zulässigen Höchstgeschwindigkeiten von $60 \mathrm{~km} / \mathrm{h}$ am Baustellenbeginn von den meisten Fahrern überschritten. So fuhren in beiden Fahrtrichtungen $85 \%$ aller Fahrzeuge mit mehr als 75 km/h in die Bereiche der Fahrstreifenverschwenkungen ein. $15 \%$ überschritten die zulässige Höchstgeschwindigkeit sogar um mindestens 35 km/h (Tabelle 4-1).
Trotz der Reduzierung der Geschwindigkeiten durch die gelbe Beschilderung fuhren weiterhin $85 \%$ der Fahrzeuge in Fahrtrichtung 1 mindestens $12 \mathrm{~km} / \mathrm{h}$ und in Fahrtrichtung $210 \mathrm{~km} / \mathrm{h}$ zu schnell. Die Hälfte der Fahrer fuhr mindestens $18 \mathrm{~km} / \mathrm{h}$ zu schnell.

\section{Querschnittsmessungen}

Die Ergebnisse der lokalen Messungen an den in Kapitel 3.4.2 angegebenen Querschnitten bestätigen die Erkenntnisse aus den Geschwindigkeitsprofilen. Im Gegensatz zu den Ergebnissen der Nachfolgefahrten erfolgte bei den Querschnittsmessungen eine nach Fahrstreifen differenzierte Betrachtung der Geschwindigkeiten. Die bisher betrachteten Kenngrößen wurden dabei um die Angaben der minimalen und maximalen beobachteten Geschwindigkeiten ergänzt. Die Darstellungen der verschiedenen Werte entspricht derjenigen in Abbildung 4-7. Während die $v_{50}$ unter Angabe des Wertes an der Basis als Balken dargestellt ist, werden die Größen $v_{15}$ und $v_{85}$ aus der unteren und oberen Begrenzung der hellgrauen Box ersichtlich. Die minimalen und maximalen Geschwindigkeiten werden durch die Linien am unteren bzw. oberen Ende markiert.

Die Ergebnisse der lokalen Geschwindigkeitsmessungen sind in Abbildung 4-8 beispielhaft für die Fahrtrichtung 
Tabelle 4-1:

Kenngrößen der Geschwindigkeitsprofile vor der Fahrstreifenverschwenkung am Baustellenbeginn im weißen und gelben Beschilderungszustand

\begin{tabular}{|c|c|c|c|c|}
\hline & \multicolumn{2}{|c|}{ Beschilderung } & \multicolumn{2}{|c|}{$\begin{array}{l}\text { Geschwindigkeitsreduktion } \\
\text { im Zustand Gelb }\end{array}$} \\
\hline & $\begin{array}{l}\text { weiß } \\
{[\mathrm{km} / \mathrm{h}]}\end{array}$ & $\begin{array}{c}\text { gelb } \\
{[\mathrm{km} / \mathrm{h}]}\end{array}$ & $\begin{array}{c}\mathrm{v} \\
{[\mathrm{km} / \mathrm{h}]}\end{array}$ & $\begin{array}{c}\text { Anteil } \\
\text { [\%] }\end{array}$ \\
\hline \multicolumn{5}{|c|}{ Fahrtrichtung 1} \\
\hline $\mathrm{v}_{15}$ & 75,1 & 72,8 & 2,3 & 3 \\
\hline$v_{50}$ & 84,5 & 81,9 & 2,7 & 3 \\
\hline$v_{85}$ & 95,3 & 88,5 & 6,8 & 7 \\
\hline \multicolumn{5}{|c|}{ Fahrtrichtung 2} \\
\hline$v_{15}$ & 76,5 & 70,3 & 6,2 & 8 \\
\hline$v_{50}$ & 85,7 & 78,0 & 7,7 & 9 \\
\hline$v_{85}$ & 97,9 & 91,1 & 6,9 & 7 \\
\hline
\end{tabular}

1 dargestellt. Daraus geht hervor, dass sich im Gegensatz zu den Nachfolgefahrten auch die $v_{50}$ zwischen den beiden betrachteten Zeiträumen unterschieden. Im Zeitraum Gelb lagen diese im Bereich der ersten gelben Fahrstreifentafel um $10 \%$ unter den Werten bei weißer Beschilderung. Allerdings nähern sich die Werte zur Baustelle hin wieder an, so dass die $v_{50}$ auf dem rechten Fahrstreifen dort nur noch etwa $5 \%$ und auf dem linken Fahrstreifen $3 \%$ unter dem Wert des Zustands weiß lagen. Dem entgegen stehen die Ergebnisse der Gegenrichtung. Dort liegen die v50 an der ersten Fahrstreifentafel im Zustand Gelb über den Ergebnissen bei weißer Beschilderung. Am Beginn der Baustelle liegen die $v_{50}$ dieser Fahrtrichtung allerdings mit Differenzen von bis zu 14 km/h (linker Fahrstreifen) etwa 15 \% unter dem bei weißer Beschilderung gemessenen Wert. Dieser Rückgang ist bei den Geschwindigkeitsprofilen weniger stark ausgeprägt. Die Ergebnisse der lokalen Geschwindigkeitsmessungen in Fahrtrichtung 2 sind in Anhang D.2 enthalten.

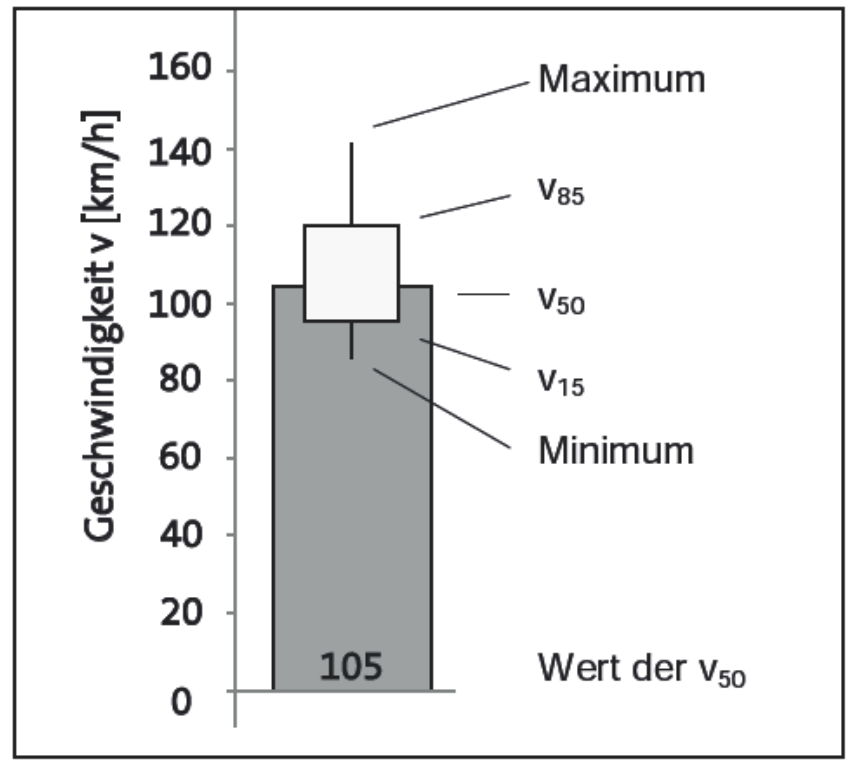

Abbildung 4-7:

in den Diagrammdarstellungen angegebene Geschwindigkeitskenngrößen der Querschnittsmessungen 


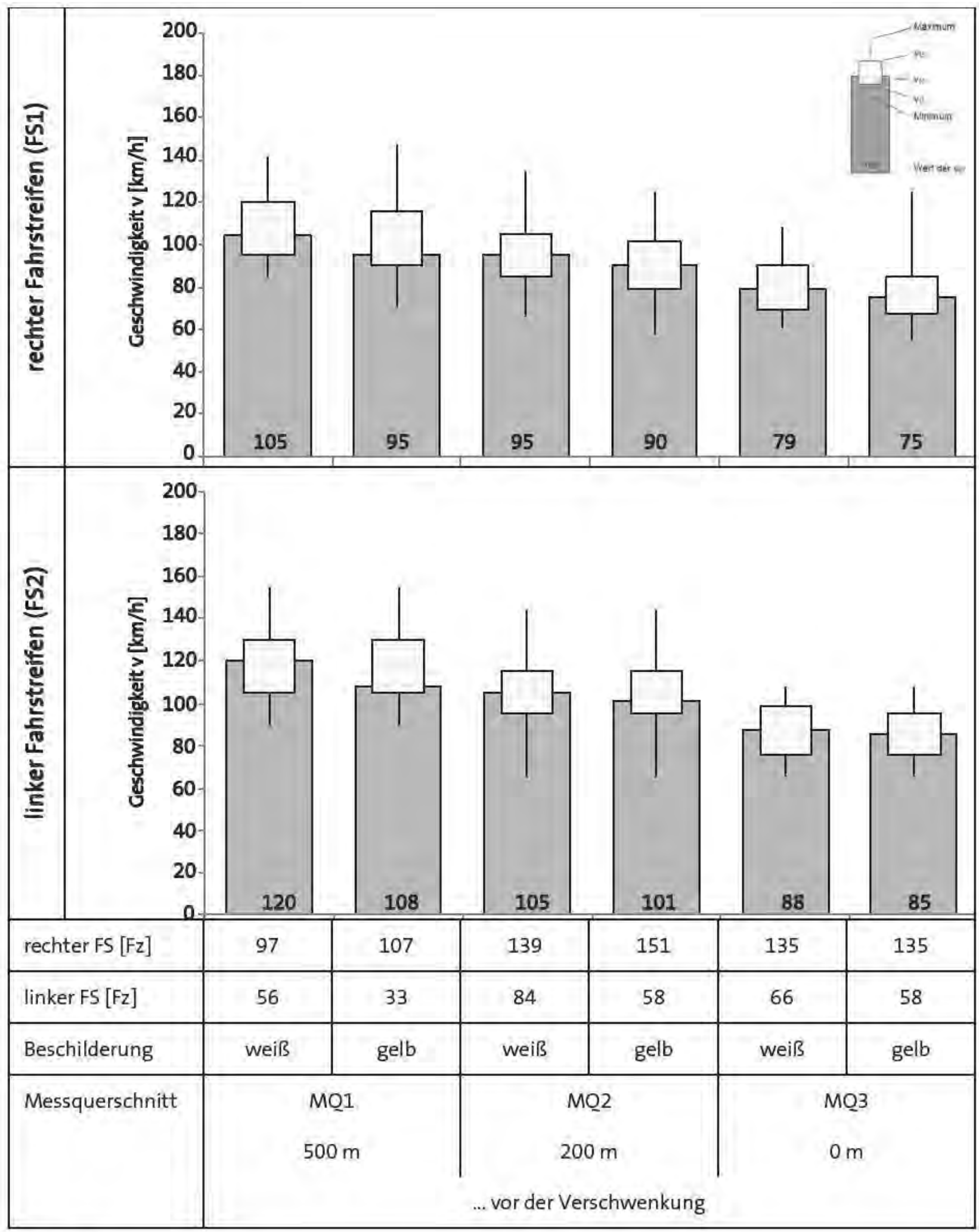

Abbildung 4-8:

Kenngrößen der Geschwindigkeitsverteilung an den Messquerschnitten MQ im Annäherungsbereich der Fahrtrichtung 1 [km/h] 
Neben den geringeren Geschwindigkeiten am Baustellenbeginn nahm ab 200 m vor der ersten Fahrstreifenverschwenkung der Fahrtrichtung 2 auch der Anteil der Linksfahrer ab (Anhang F). Aus diesem Rückgang kann insbesondere im Hinblick auf den hohen Anteil von Unfällen durch Zusammenstöße mit seitlich in gleicher Richtung fahrenden Fahrzeugen, welche in Autobahnbaustellen und vor allem in Bereichen von Fahrstreifenverschwenkungen auftreten, eine Erhöhung der Verkehrssicherheit in diesen Bereichen abgeleitet werden.

\subsection{Baustellenende}

\section{Geschwindigkeitsprofile}

Aus den Geschwindigkeitsprofilen am Baustellenende lässt sich für den Bereich der Fahrstreifenverschwenkungen ebenfalls ein positiver Effekt der modifizierten
Beschilderung ableiten. In diesen Bereichen betrug die zulässige Höchstgeschwindigkeit jeweils 60 km/h. Während die $v_{15}$ und $v_{50}$ beider Zustände vergleichbar waren, konnten die $v_{85}$ in beiden Fahrtrichtungen um etwa $4 \mathrm{~km} / \mathrm{h}$ reduziert werden. Die Werte lagen am Beginn der Fahrstreifenverschwenkung (Fahrtrichtung 2) etwa $3 \%$ und vor der Überleitung (Fahrtrichtung 1) $4 \%$ unter dem Wert bei weißer Beschilderung (Tabelle 4-2).

Während die Geschwindigkeitskenngrößen $v_{15}$ und $v_{50}$ gegenüber dem davor liegenden Baustellenbereich vor der Fahrstreifenüberleitung am Baustellenende im weißen und im gelben Beschilderungszustand in gleichem Maß um etwa $6 \%$ zurückgingen, blieb der Wert der $v_{85}$ im Zustand Weiß unverändert. Im Zustand Gelb dagegen nahm auch der Wert der $v_{85}$ um $6 \%$ ab. Demnach konnte durch die Ausstattung des Bereichs vor der Überleitung der Fahrstreifen mit gelben Überleitungstafeln und der

\section{Tabelle 4-2:}

Kenngrößen der Geschwindigkeitsprofile vor der Fahrstreifenverschwenkung am Baustellenende im weißen und gelben Beschilderungszustand

\begin{tabular}{|c|c|c|c|c|}
\hline & \multicolumn{2}{|c|}{ Beschilderung } & \multicolumn{2}{|c|}{$\begin{array}{l}\text { Geschwindigkeitsreduktion } \\
\text { im Zustand Gelb }\end{array}$} \\
\hline & $\begin{array}{c}\text { weiß } \\
{[\mathrm{km} / \mathrm{h}]}\end{array}$ & $\begin{array}{c}\text { gelb } \\
{[\mathrm{km} / \mathrm{h}]}\end{array}$ & $\begin{array}{c}\mathrm{v} \\
{[\mathrm{km} / \mathrm{h}]}\end{array}$ & $\begin{array}{c}\text { Anteil } \\
{[\%]}\end{array}$ \\
\hline \multicolumn{5}{|c|}{ Fahrtrichtung 1} \\
\hline$v_{15}$ & 78,1 & 76,8 & 1,2 & 2 \\
\hline$v_{50}$ & 86,5 & 88,3 & $-1,8$ & -2 \\
\hline$v_{85}$ & 102,1 & 97,6 & 4,5 & 4 \\
\hline \multicolumn{5}{|c|}{ Fahrtrichtung 2} \\
\hline$v_{15}$ & 84,0 & 83,1 & 0,9 & 1 \\
\hline$v_{50}$ & 93,7 & 94,0 & $-0,3$ & 0 \\
\hline$v_{85}$ & 106,0 & 102,3 & 3,7 & 3 \\
\hline
\end{tabular}




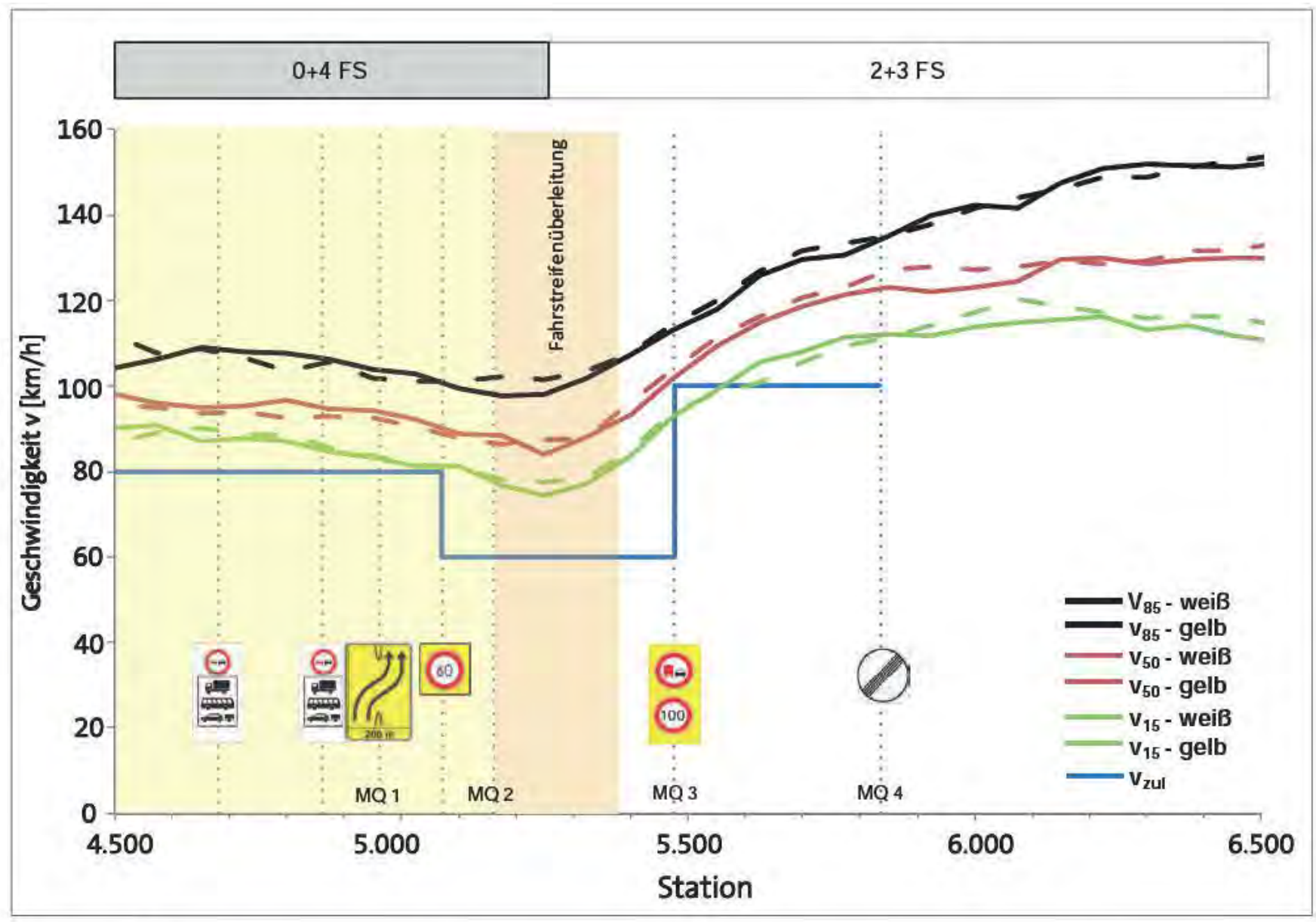

Abbildung 4-9:

Verläufe der Geschwindigkeitskenngrößen v15, v50 und v85 am Baustellenende der Fahrtrichtung 1 nach Beschilderungsart

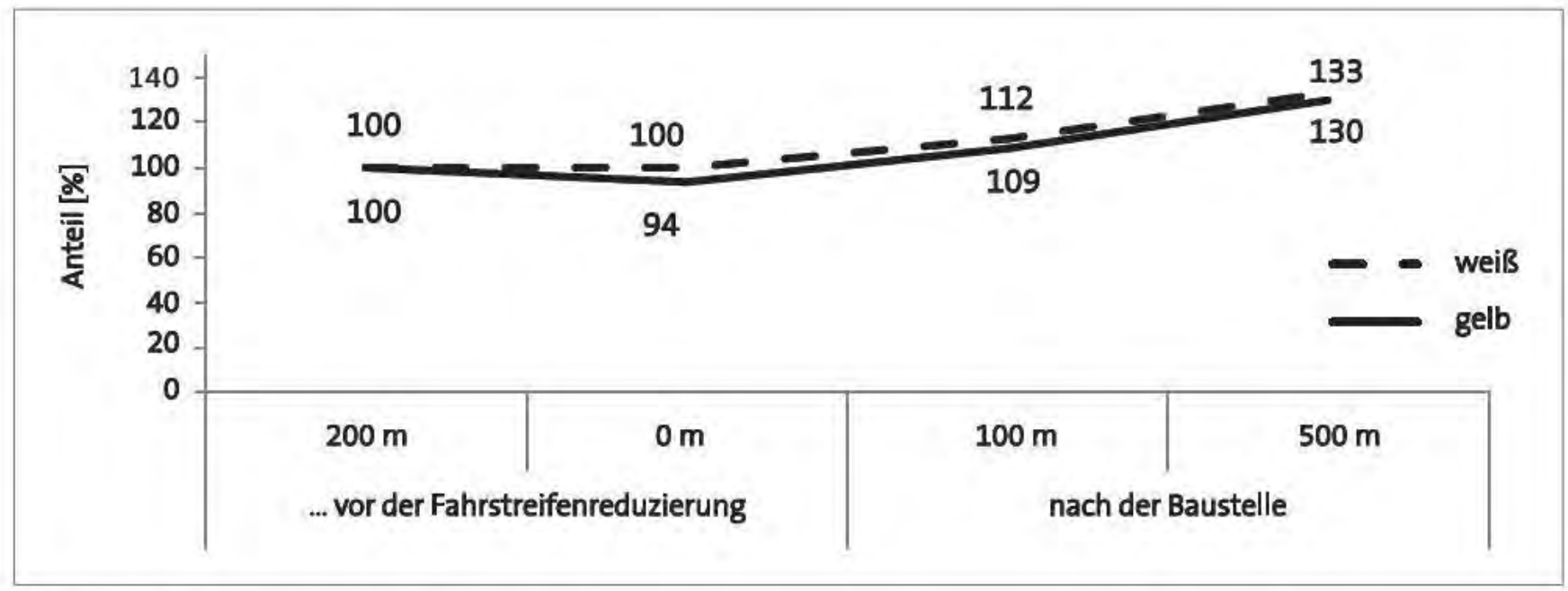

Abbildung 4-10:

Anstieg der v85 im Annäherungsbereich der Fahrtrichtung 1 
Hervorhebung des Zeichens 274-56 $\left(\mathrm{v}_{\mathrm{zul}}=60 \mathrm{~km} / \mathrm{h}\right)$ durch eine gelbe Hintergrundtafel eine Reduzierung der Geschwindigkeiten bei schnellen Fahrern erreicht werden.

Anders verhielt es sich am Baustellenende der Fahrtrichtung 2. Hier wurden die Fahrstreifen lediglich auf der eigenen Fahrbahn mit einer zulässigen Höchstgeschwindigkeit von $80 \mathrm{~km} / \mathrm{h}$ verschwenkt. Zudem erfolgte nach Baustellenende eine Addition von zwei auf drei Fahrstreifen. Der Verlauf der $v_{15}$ und $v_{85}$ deutet auf ein späteres Einsetzen der Beschleunigung bei gelber Beschilderung hin. Während im Zustand Weiß die Geschwindigkeiten bereits am Beginn der Fahrstreifenverschwenkung zunahmen, setzt im Zustand Gelb der Anstieg der beiden genannten Kenngrößen erst auf Höhe des gelb hinterlegten Zeichens 274-60 ( $\left.v_{z u l}=100 \mathrm{~km} / \mathrm{h}\right)$ ein. Demnach bewirkte die Hervorhebung dieses Verkehrszeichens vor allem bei langsamen (Schwerverkehr) und schnellen Fahrzeugen eine Verschiebung der Beschleunigung hinter dem kritischen Bereich der Fahrstreifenverschwenkung.

Einen Einfluss auf die Bandbreite der gefahrenen Geschwindigkeiten am Baustellenende kann der gelben Beschilderung nicht nachgewiesen werden. Zwar ist die Differenz der drei betrachteten Geschwindigkeitskenngrößen in Fahrtrichtung 2 im Zustand Gelb geringer, jedoch stellt sich diese Verringerung in der Gegenrichtung nicht ein.

\section{Querschnittsmessungen}

In den Ergebnissen der Querschnittsmessungen stellten sich vor der Fahrstreifenüberleitung in Fahrtrichtung 1 zwischen den beiden untersuchten Beschilderungszuständen keine Unterschiede in den Geschwindigkeitskenngrößen ein. Dagegen lagen insbesondere die $v_{85}$ an den beiden Messquerschnitten vor der Fahrstreifenverschwenkung der Fahrtrichtung 2 im Zustand Gelb z. T. deutlich unter den Werten des Zustands Weiß.

Insbesondere im Bereich der Überleitung der Fahrstreifen am Baustellenende (Fahrtrichtung 1) fuhren im Zustand Gelb anteilig weniger Fahrzeuge auf dem Überholfahrstreifen. Erst im Bereich der Aufhebung aller Streckenver- bote unterschieden sich die Anteilswerte der Linksfahrer an der Gesamtverkehrsstärke nicht von denen des weiBen Zustands (Anhang F).

\subsection{Anschlussstelle innerhalb der Baustelle}

Die in Fahrtrichtung 2 auch während des Bauzeitraums geöffnete Anschlussstelle wies keine verkürzten Verzögerungs- und Beschleunigungsstreifen, wie sie in Autobahnbaustellen häufig anzutreffen sind, auf. Lediglich die Breite dieser Fahrstreifen wurde reduziert.

Die im Bereich der Einfahrt angeordnete und im Zustand "gelb“ modifizierte Beschilderung sollte auf die besondere Situation einfahrender Fahrzeuge hinweisen und durch die Verringerung der zulässigen Höchstgeschwindigkeit auf $60 \mathrm{~km} / \mathrm{h}$ den zufahrenden Fahrzeugen ein besseres Einfädeln auch bei hohen Verkehrsstärken ermöglichen. Insgesamt sollte so ein Verkehrsablauf mit weniger Störungen gewährleistet werden.

Neben der Modifizierung der Beschilderung war im Zustand Gelb das Zeichen 274-56 $\left(\mathrm{v}_{\text {zul }}=60 \mathrm{~km} / \mathrm{h}\right)$ erst nach dem Verzögerungsstreifen der Ausfahrt angeordnet. Zuvor galt diese zulässige Höchstgeschwindigkeit für den gesamten Bereich der Anschlussstelle. Deshalb waren die Geschwindigkeitskenngrößen der Querschnittsmessungen nicht miteinander vergleichbar. Die Bewertung des Geschwindigkeitsverhaltens erfolgt deshalb auf Grundlage der Geschwindigkeitsprofile der Nachfolgefahrten. Aus deren Kenngrößen ließ sich ein Einfluss der Beschilderung feststellen.

Bei weißer Beschilderung konnte vor allem ein Rückgang der Geschwindigkeit bei den schnellen Fahrzeugen $\left(v_{85}\right)$ festgestellt werden. Allerdings erfolgte diese Reduzierung auf einem für Baustellenbereiche hohen Geschwindigkeitsniveau von $105 \mathrm{~km} / \mathrm{h}$ auf $100 \mathrm{~km} / \mathrm{h}$. Demnach fuhren im Bereich der Anschlussstelle noch immer $15 \%$ aller Fahrer mindestens $40 \mathrm{~km} / \mathrm{h}$ zu schnell. Die Kenngrößen $v_{15}$ und $v_{50}$ zeigten keine veränderungen. Die zulässige Höchstgeschwindigkeit von $60 \mathrm{~km} / \mathrm{h}$ wurde von $85 \%$ aller beobachteten Fahrzeuge um mindestens 


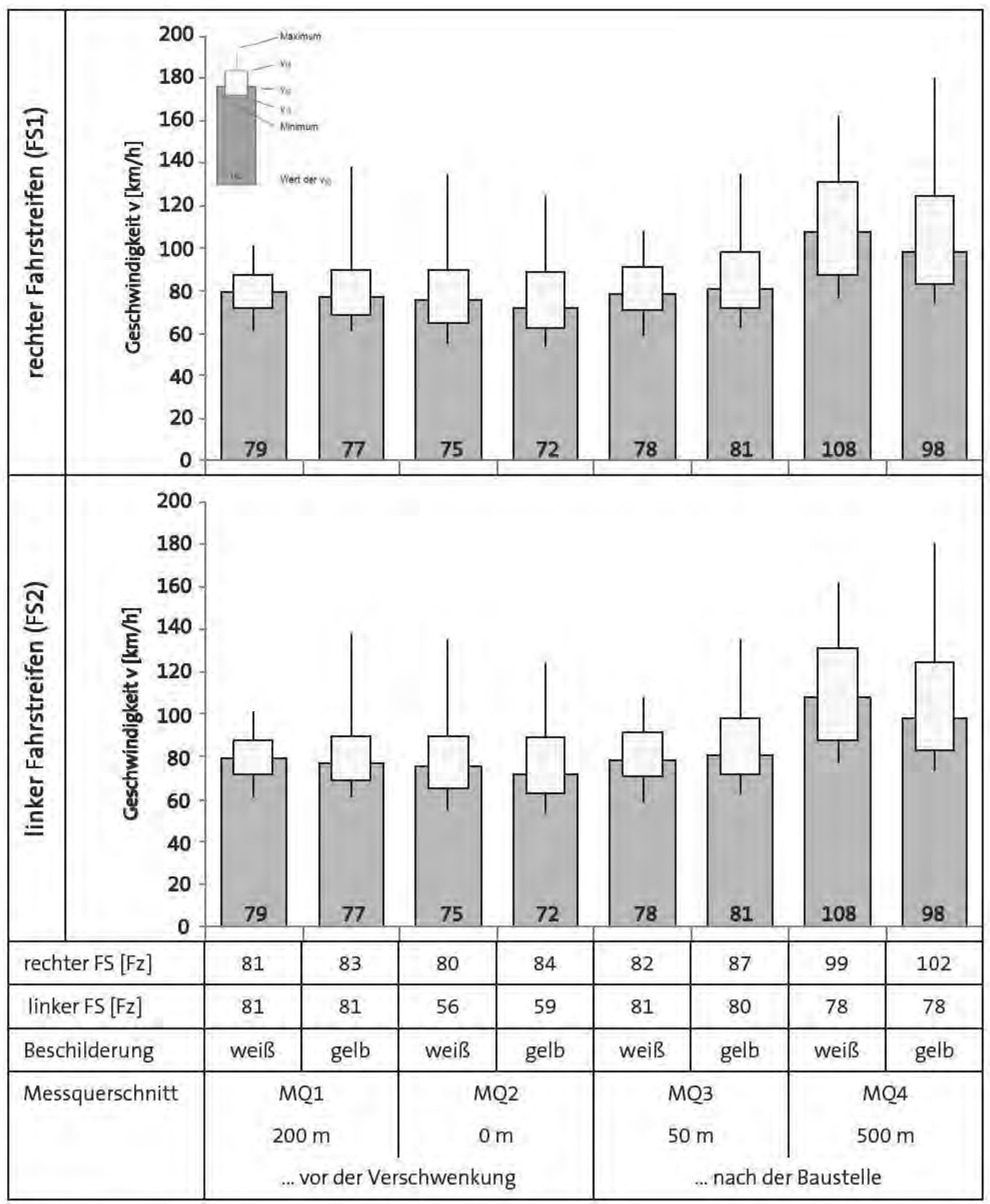

Abbildung 4 11:

Kenngrößen der Geschwindigkeitsverteilung an den Messquerschnitten am Baustellenende der Fahrtrichtung 1 [km/h] 
$23 \mathrm{~km} / \mathrm{h}$ überschritten. Die Hälfte aller Fahrer fuhr hier mindestens $30 \mathrm{~km} / \mathrm{h}$ zu schnell.

Im Zustand Gelb gingen die Geschwindigkeiten Infolge der Reduzierung der zulässigen Höchstgeschwindigkeit auf $60 \mathrm{~km} / \mathrm{h}$ etwas stärker zurück. Dieser Rückgang konnte vor allem in den $v_{15}$ und $v_{85}$ nachgewiesen werden. Die $v_{85}$ verringerte sich um fast $9 \%$ von $104 \mathrm{~km} / \mathrm{h}$ auf etwa $95 \mathrm{~km} / \mathrm{h}$. Bei der $\mathrm{v}_{15}$ war eine Reduzierung um ca. $6 \mathrm{~km} / \mathrm{h}$ zu verzeichnen. Wie bereits bei der weißen Beschilderung spiegelten sich auch hier die Geschwindigkeitsreduzierungen nicht in der $v_{50}$ wider. Die mit einer gelben Hintergrundtafel hervorgehobene Geschwindigkeitsre- duzierung wirkt sich demnach vor allem auf schnelle und langsame Fahrzeuge aus, führt aber ebenfalls nicht zu einer verbesserten Akzeptanz der zulässigen Höchstgeschwindigkeit von $60 \mathrm{~km} / \mathrm{h}$ in diesem Bereich. $85 \%$ der Fahrer fahren schneller als $80 \mathrm{~km} / \mathrm{h}$.

\subsection{Wirkung der stufenweisen Anhe- bung der zulässigen Höchstge- schwindigkeit nach der Baustelle}

Bei der Untersuchung der Wirkung einer selektivgelben Beschilderung auf das Fahrverhalten, wurde zusätzlich noch in beiden Phasen (weiß und gelb) eine stufenwei-

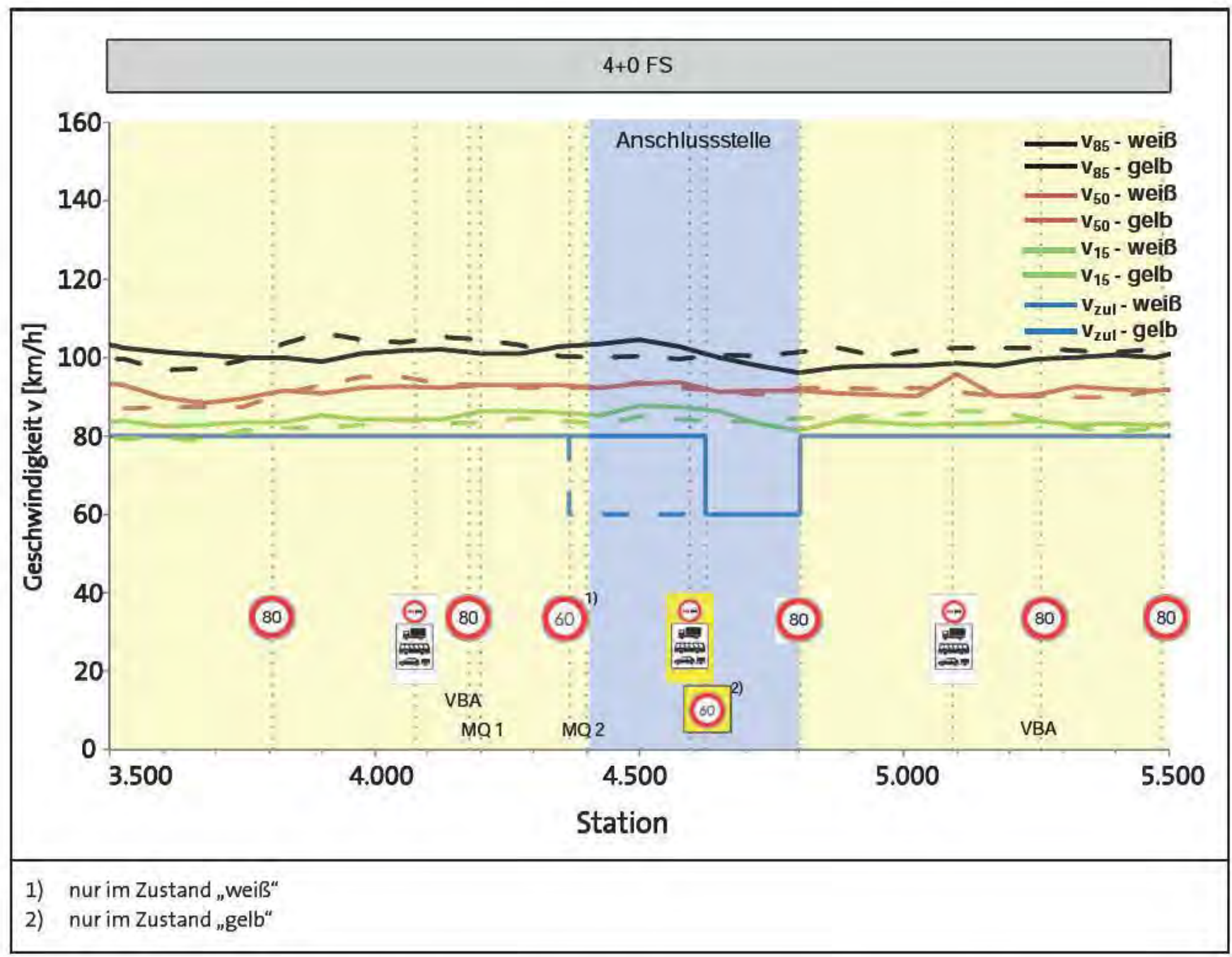

Abbildung 4-12:

Verläufe der Geschwindigkeitskenngrößen $\mathbf{v}_{15}, \mathbf{v}_{50}$ und $\mathbf{v}_{85}$ vor der Behelfsausfahrt der Fahrtrichtung 2 nach Beschilderungsart 
se Anhebung der Geschwindigkeit aus folgendem Grund geprüft: Kurz nach dem Ende einer Baustelle finden zahlreiche Verflechtungsvorgänge statt. Da hier jedoch die Begrenzung der zulässigen Höchstgeschwindigkeit infolge der Baustelle bereits aufgehoben ist, wird in diesem Bereich meist stark beschleunigt. Ziel der abgestuften Geschwindigkeitserhöhung ist es deshalb, das Geschwindigkeitsniveau unmittelbar hinter Baustellenende weiter niedrig zu halten und für einen homogeneren Geschwindigkeitsverlauf zu sorgen.

Damit die maßgebenden Geschwindigkeitskenngrößen der einzelnen Baustellen miteinander verglichen werden können, müssen gleiche Ausgangssituationen zum Baustellenende vorhanden sein. Somit wurde das Kollektiv in zwei Gruppen geteilt (siehe Tabelle 4-3):

- $\mathrm{v}_{\mathrm{zul}}=60 \mathrm{~km} / \mathrm{h}$ zum Baustellenende und

- $\mathrm{v}_{\mathrm{zul}}=80 \mathrm{~km} / \mathrm{h}$ zum Baustellenende.

Die Autobahnbaustellen bei Duisburg und Jena (Richtung Weimar) sind aufgrund des allgemein niedrigen Geschwindigkeitsniveaus (mit Überwachung) von dieser Betrachtung ausgeschlossen worden.

Da im weiteren Verlauf unterschiedliche Geschwindigkeitsbeschränkungen angeordnet waren, ließen sich die

\section{Tabelle 4-3:}

Baustellenenden - Übersicht der Charakteristika (grau hinterlegt: Untersuchungsbaustelle „Gelbe Baustelle“)

\begin{tabular}{|c|c|c|c|c|}
\hline $\begin{array}{l}\text { Autobahn } \\
\text { BAB }\end{array}$ & $\begin{array}{c}\text { Nächstgelegene } \\
\text { Stadt }\end{array}$ & Fahrtrichtung & $\begin{array}{c}\text { Führung nach } \\
\text { Baustellen- } \\
\text { ende }\end{array}$ & $\begin{array}{c}\text { Tempolimit zum } \\
\text { Baustellenende } \\
{[\mathrm{km} / \mathrm{h}]}\end{array}$ \\
\hline \multicolumn{5}{|c|}{$\mathrm{v}_{\mathrm{zul}}=60 \mathrm{~km} / \mathrm{h}$} \\
\hline A 6 & Kaiserslautern & Saarbrücken (1) & 2-streifig & $60->100->$ unbegrenzt \\
\hline \multirow{2}{*}{ A 4} & \multirow{2}{*}{ Aachen } & Köln (1) & 3-streifig & $60->$ unbegrenzt \\
\hline & & Aachen (2) & 3-streifig & $60->120$ \\
\hline A 4 & Chemnitz & Frankenberg (1) & 3-streifig & $60->130$ \\
\hline \multirow{2}{*}{ A 14} & \multirow{2}{*}{ Halle } & Leipzig (1) & 3-streifig & $60->$ unbegrenzt \\
\hline & & Magdeburg (2) & 2-streifig & $60->120->$ unbegrenzt \\
\hline \multicolumn{5}{|c|}{$\mathrm{v}_{\mathrm{zul}}=80 \mathrm{~km} / \mathrm{h}$} \\
\hline A 6 & Kaiserslautern & Mannheim (2) & 3-streifig & $80->100->130$ \\
\hline A 4 & Chemnitz & Crimmitschau (2) & 3-streifig & $80->$ unbegrenzt \\
\hline A 4 & Jena & Gera (1) & 3-streifig & $80->$ unbegrenzt \\
\hline \multicolumn{5}{|l|}{ Sonstige } \\
\hline A 4 & Jena & Weimar (2) & 2-streifig & $60->130$ \\
\hline \multirow{2}{*}{ A 59} & \multirow{2}{*}{ Duisburg } & Düsseldorf (1) & 3-streifig & $60->100$ \\
\hline & & Dinslaken (2) & 2-streifig & $60->80$ \\
\hline
\end{tabular}


Baustellen mit $v_{\text {zul }}=80 \mathrm{~km} / \mathrm{h}$ am Baustellenende nur bedingt gegenüberstellen. Deshalb werden in dieser Auswertung nur Baustellen mit einer Ausgangsgeschwindigkeit von $v_{\text {zul }}=60 \mathrm{~km} / \mathrm{h}$ am Baustellenende betrachtet.

Abbildung 4-13 zeigt hierzu die $v_{85}$ am Baustellenende im Vergleich. Auch bei der Baustelle BAB A14 Halle (Fahrtrichtung Magdeburg) wurde die zulässige Höchstgeschwindigkeit schrittweise angehoben. Es ist zu erkennen, dass das Geschwindigkeitsniveau im Bereich von $\mathrm{v}_{\mathrm{zul}}=60 \mathrm{~km} / \mathrm{h}$ recht unterschiedlich ist. Lediglich die Baustelle bei Aachen weist ähnlich hohe Geschwindigkeiten auf. Im weiteren Verlauf nimmt die $v_{85}$ bei fast allen Baustellen im gleichen Maße zu. Nur bei der Baustelle auf der BAB A4 (Chemnitz, Richtung Frankenberg) ist ein stärkerer Geschwindigkeitsanstieg zu sehen. Dies könnte jedoch auch an der vergleichsweise niedrigen Ausgangsgeschwindigkeit von $v_{85}=75 \mathrm{~km} / \mathrm{h}$ liegen. Daher kann hieraus keine Tendenz abgeleitet werden.

Im nächsten Schritt wird die Homogenität der Geschwindigkeiten, abgeleitet aus der Differenz der Kenngrößen $\mathrm{v}_{85}$ und $\mathrm{v}_{50}$, in diesem Bereich betrachtet (siehe Abbildung 4-14). Hierbei kann ein positiver Effekt festgestellt werden. Die Baustellen mit einer stufenweisen Anhebung der Geschwindigkeit liegen im „kritischen“ Bereich (Station $0+000$ bis $0+500$ ) im Mittel deutlich unter den restlichen Baustellen. Demzufolge war in dem Abschnitt, zu dem generell die meisten Verflechtungsvorgängen gehören, bei schrittweiser Anhebung der Geschwindigkeit ein homogenerer Geschwindigkeitsverlauf festzustellen. Aus diesem Grund wird eine abgestufte Erhöhung der zulässigen Geschwindigkeit empfohlen.

\subsection{Zusammenfassung}

Aus den Untersuchungsergebnissen ließen sich keine Wirkungen auf das Abstandsverhalten der Fahrer zum vorausfahrenden Fahrzeug ableiten. Jedoch konnte ein Einfluss der modifizierten Beschilderung auf das Fahrverhalten festgestellt werden. Neben Unterschieden in den Belegungsanteilen des Überholfahrstreifens an einigen Querschnitten, ließ sich ein Einfluss der Gelben Beschilderung vor allem bei den Geschwindigkeiten nachweisen.
- Im Annäherungsbereich wurden durch die Anordnung der gelben Beschilderung die Geschwindigkeiten frühzeitig reduziert. Am Baustellenbeginn vor der Fahrstreifenverschwenkung konnten die $v_{85}$ in beiden Fahrtrichtungen um etwa $7 \mathrm{~km} / \mathrm{h}$ reduziert und die Ergebnisse aus BAIER et al. (2006) bestätigt werden.

- Im Annäherungsbereich der Fahrtrichtung 2, die aufgrund der Fahrstreifenreduzierung eine frühere Reduzierung der zulässigen Höchstgeschwindigkeit aufwies, konnte neben der $\mathrm{v}_{85}$ auch eine Reduzierung der Kenngrößen $v_{15}$ und $v_{50}$ nachgewiesen werden. Demnach konnten in dieser Fahrtrichtung die Geschwindigkeiten aller Fahrzeuge um ca. $7 \mathrm{~km} / \mathrm{h}$ reduziert werden. Bereits in BAIER er al. (2006) wurde im Sinne einer größeren Geschwindigkeitsreduzierung am Baustellenbeginn ein gegenüber den RSA 95 früherer Beginn der Geschwindigkeitsreduzierung empfohlen.

- Am Baustellenende lässt sich insbesondere aus Kenngrößen der Geschwindigkeitsprofile eine Tendenz zum späteren Beschleunigen ableiten. So nahmen die Geschwindigkeiten in diesen Bereichen im Gegensatz zur weißen Beschilderung erst nach der Fahrstreifenverschwenkung zu.

- Im Bereich von Geschwindigkeitsbeschränkungen auf $v_{\text {zul }}=60 \mathrm{~km} / \mathrm{h}$ konnten sowohl am Baustellenende, als auch im Bereich der Anschlussstelle im Vergleich zum Zustand Weiß die gefahrenen Geschwindigkeiten erkennbar verringert werden.

- Die größte Auswirkung hatte die gelbe Beschilderung auf die schneller Fahrenden. Gegenüber der weißen Beschilderung ging die $v_{85}$ meist deutlicher zurück als die $v_{50}$.

- Die gelbe Beschilderung führt aber nicht zu einer wesentlich besseren Akzeptanz der zulässigen Höchstgeschwindigkeiten. Die Hälfte aller beobachteten Fahrzeuge überschritt die in den Überleitungen gültigen Geschwindigkeitsbeschränkungen von $60 \mathrm{~km} / \mathrm{h}$ um mindestens $20 \mathrm{~km} / \mathrm{h}$.

- Die stufenweise Anhebung der zulässigen Höchstgeschwindigkeit nach dem Ende der Baustelle verringerte die Bandbreite der Geschwindigkeiten. Ein Effekt auf die Höhe der gefahrenen Geschwindigkeiten und insbesondere auf die Beschleunigung ließ sich nicht nachweisen. 


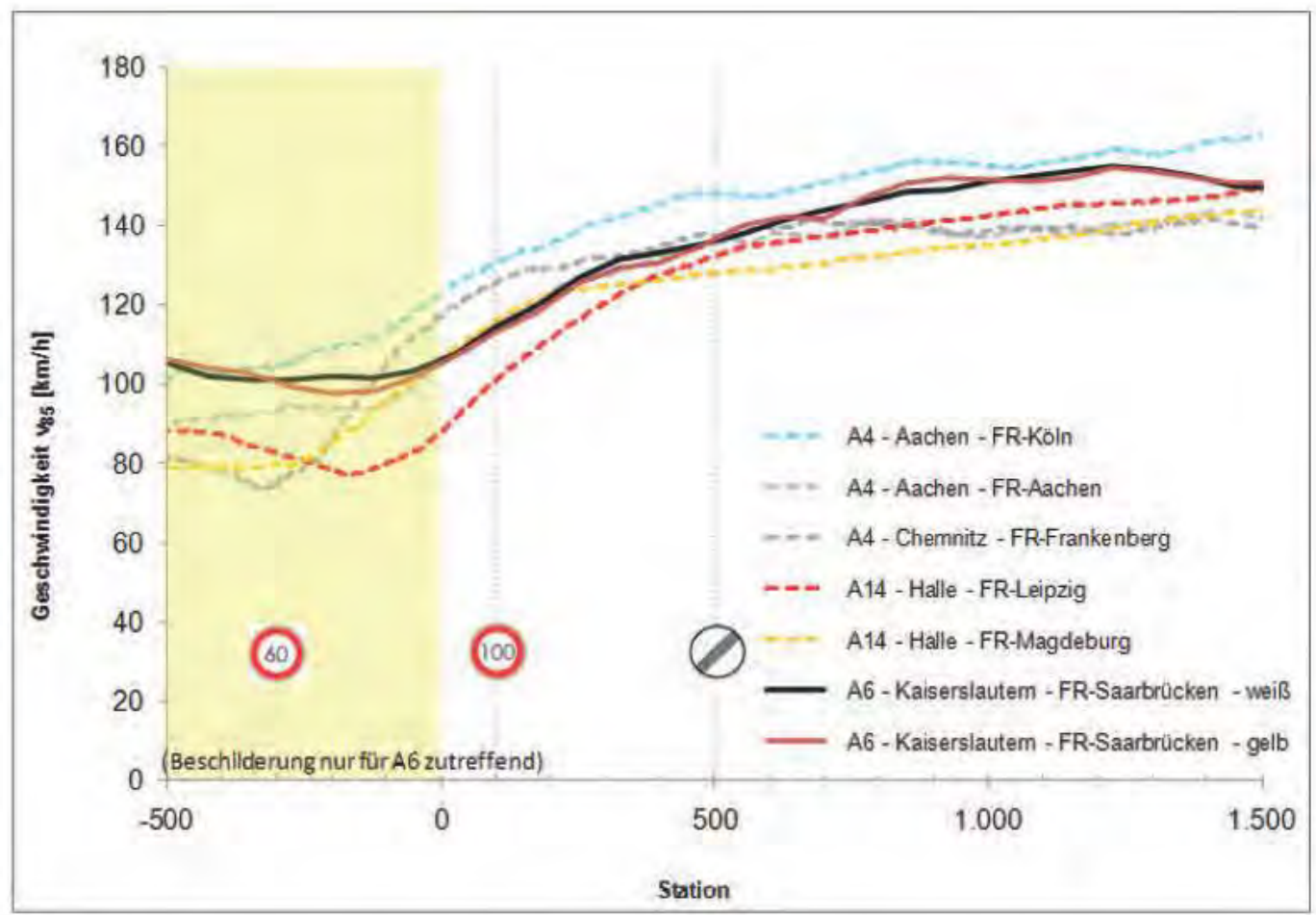

Abbildung 4-13:

Verlauf der $\mathrm{v}_{85}$ am Baustellenende der Untersuchungsbaustelle und von Baustellen der abgeschlossenen Untersuchung (QSV, 2010)

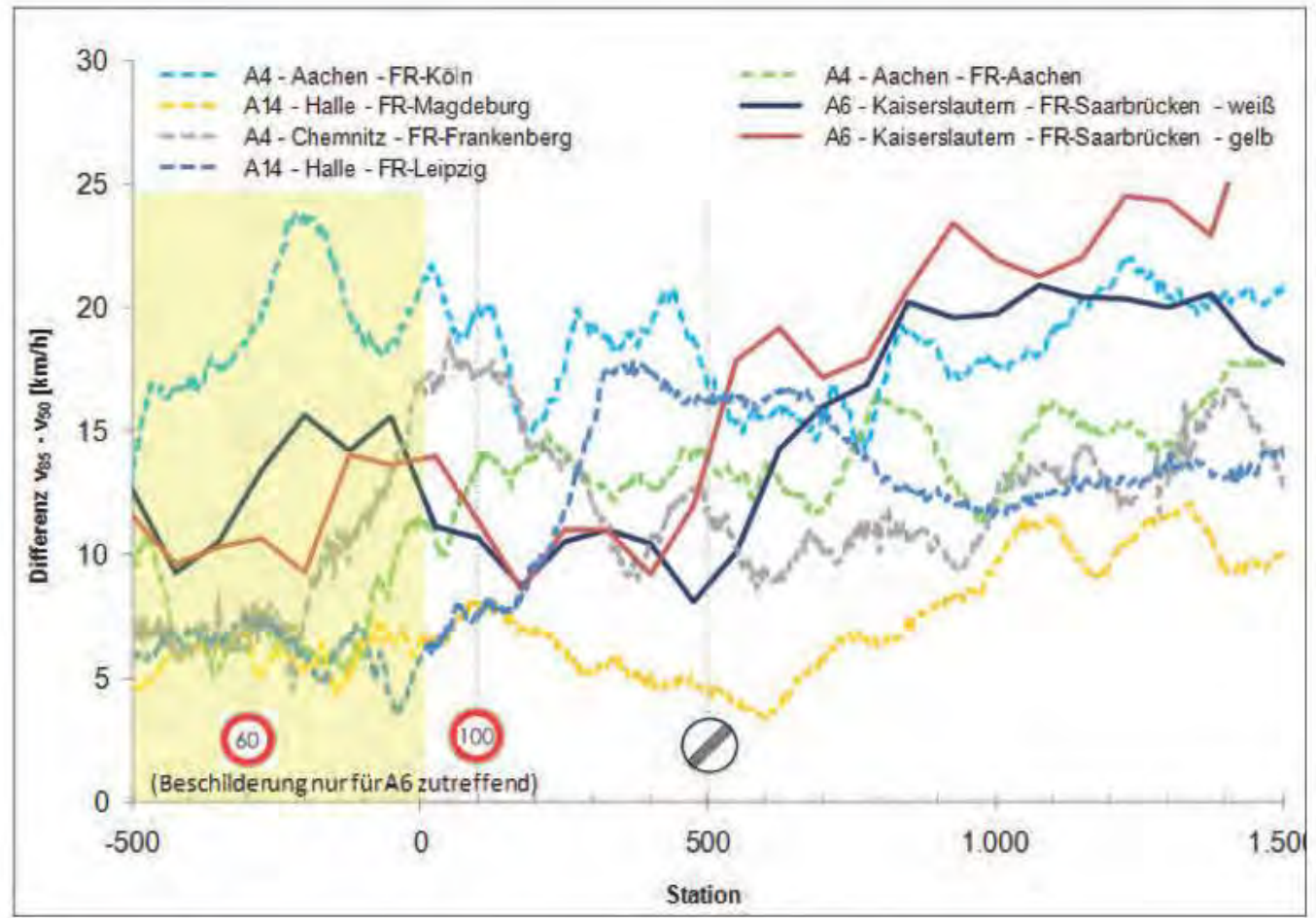

Abbildung 4-14:

Homogenität der Geschwindigkeit am Baustellenende 


\section{Unfallgeschehen}

Im Bereich der Autobahnbaustelle ${ }^{3}$ wurden im Untersuchungszeitraum 23 Unfälle gemeldet. 10 dieser Unfälle ereigneten sich in Zeiten der Einrichtung oder des Abbaus der Baustelle und 13 Unfälle während des Bauzeitraums.

Alle Unfälle hatten Sachschaden zur Folge, zwei davon ereigneten sich mit schwerem Sachschaden. Die Unfallrate, die aufgrund des geringen Unfallkollektivs keine statistische Aussagekraft besitzt, liegt im Bereich des in OSV (2010) ermittelten Wertes für Baustellen auf Autobahnen $\left(0,9 \mathrm{U} / 106 \mathrm{Kfz}^{*} \mathrm{~km}\right)$. Die Unfallkostenraten lagen deutlich unter den Werten der früheren Untersuchung.

Insgesamt ereigneten sich 9 der 23 Unfälle auf nasser bzw. winterglatter Fahrbahn bei Dunkelheit. Diese Zahlen sind vor dem Hintergrund des Bauzeitraums, welcher sich von September bis Dezember erstreckte, zu bewerten. Alle Unfälle bei Nässe und Dunkelheit ereigneten sich in den Monaten November und Dezember, die durch häufige Regenfälle und Wintereinbruch gekennzeichnet waren.

Werden ausschließlich die Zeitbereiche betrachtet, in denen die Baustelle unverändert mit der gleichen Beschilderung und Verkehrsführung bestand, ereigneten sich bei der Gestaltung nach RSA 95 in den Monaten September/Oktober drei Unfälle. Im Zeitraum der folgenden gelben Beschilderung wurden 10 Unfälle registriert. Davon stehen sieben im Zusammenhang mit nassen oder winterglatten Straßen.

Zusammenhänge zwischen dem Unfallgeschehen und der Ausführung der Beschilderung lassen sich nicht ableiten.
Tabelle 5-1:

Ausprägung der Unfallumstände in der untersuchten Baustelle im Vergleich zu OSV (2010)

\begin{tabular}{|l|c|c|c|}
\hline & $\begin{array}{c}\text { Auto- } \\
\text { bahn- } \\
\text { baustelle }\end{array}$ & \multicolumn{2}{|c|}{ Fahrtrichtung } \\
\hline Unfallanzahl & & 13 & 10 \\
\hline Wochenende & $17 \%$ & $23 \%$ & $0 \%$ \\
\hline $\begin{array}{l}\text { Spitzenstunde } \\
\text { Dämmerung / } \\
\text { Dunkelheit }\end{array}$ & $39 \%$ & $31 \%$ & $20 \%$ \\
\hdashline \begin{tabular}{l} 
Nässe / Glätte \\
\hline
\end{tabular} & $19 \%$ & $54 \%$ & $40 \%$ \\
\hline
\end{tabular}

\section{Tabelle 5-2:}

Unfälle nach Abschnitten der untersuchten Baustelle im baulich unveränderten Zeitraum

\begin{tabular}{|c|c|c|c|c|c|}
\hline \multirow{2}{*}{\multicolumn{2}{|c|}{$\begin{array}{l}\text { Beschilderung } \\
\text { Fahrtrichtung }\end{array}$}} & \multicolumn{2}{|c|}{ weiß } & \multicolumn{2}{|c|}{ gelb } \\
\hline & & 1 & 2 & 1 & 2 \\
\hline \multicolumn{2}{|c|}{ Unfallanzahl } & 2 & 1 & 5 & 5 \\
\hline \multirow{5}{*}{ 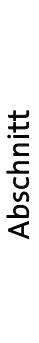 } & Annäherung & & 1 & 3 & \\
\hline & $\begin{array}{l}\text { Fahrstreifenverschwenkung } \\
\text { am Baustellenbeginn }\end{array}$ & & & 1 & 1 \\
\hline & Strecke & 2 & & 1 & 2 \\
\hline & $\begin{array}{l}\text { Fahrstreifenverschwenkung } \\
\text { am Baustellenende }\end{array}$ & & & & \\
\hline & Nachlauf (500 m) & & & & 2 \\
\hline
\end{tabular}




\section{Zusammenfassung und Empfehlungen}

In den Untersuchungen von QSV (2010) wurden Bereiche von Autobahnbaustellen mit Besonderheiten in Verkehrsführung (Fahrstreifenverschwenkungen) oder -ablauf (Anschlussstellen) als besonders unsicher erkannt. Häufig unterschätzen die Fahrzeugführer die spezifischen Gefahren solcher Bereiche. In der Vergangenheit gab es bereits verschiedene Untersuchungen, die sich mit dem optischen Hervorheben von Beschilderung vor allem in Auto $\neg$ bahnbaustellen befassten. Durch das Hervorheben soll beim Fahrzeugführer die Aufmerksamkeit für die besondere Situation der Baustelle oder des jeweiligen Baustellenbereichs erhöht werden. Dabei hat sich die Verwendung selektivgelber Typ-3-Reflexfolie als besonders geeignet erwiesen (BAIER et al. 2006).

Ziel der vorliegenden Untersuchung war die Überprüfung der Wirkung des Einsatzes selektivgelber Beschilderung vor und innerhalb von Baustellenbereichen mit geringer Verkehrssicherheit. Die Untersuchung wurde in zwei Untersuchungsabschnitte unterteilt. Zunächst erfolgte die Einrichtung der Baustelle nach RSA 95 mit Verkehrszeichen, Fahrstreifentafeln und Baken auf weißem Grund. Dabei wurde Typ-2-Reflexfolie verwendet. Bei unveränderter Gestaltung der Baustellengeometrie wurden im zweiten Untersuchungsabschnitt die mit den Untersuchungsbereichen im Zusammenhang stehenden Verkehrszeichen, Fahrstreifentafeln und Baken modifiziert. Während bei Fahrstreifentafeln und Baken die sonst weißen Flächen in Gelb ausgeführt wurden, wurden die Verkehrszeichen mit Geschwindigkeitsbeschränkungen oder Überholverboten mit einer gelben Hintergrundtafel versehen.

Insgesamt ließen sich positive Auswirkungen der gelben Beschilderung auf die Geschwindigkeiten am Baustellenbeginn erkennen. Bei Einfahrt in die Verschwenkung bzw. Überleitung fuhren die Fahrzeuge im Zustand Gelb im Mittel $7 \mathrm{~km} / \mathrm{h}$ langsamer. Gleichzeitige nahm insbesondere in Fahrtrichtung 2 der Anteil von Fahrzeugen auf dem Überholfahrstreifen ab. Auch am Baustellenende konnte vor allem vor der Fahrstreifenüberleitung ein Rückgang der Linksfahrer beobachtet werden. Außerdem setzte bei gelber Beschilderung die Beschleunigung im Gegensatz zur weißen Beschilderung erst nach den Verschwenkungen ein.

Da sich in Bereichen von Fahrstreifenverschwenkungen und -überleitungen häufig Unfälle durch seitliches Streifen von in gleicher Richtung fahrenden Fahrzeugen ereignen, kann aus den Ergebnissen eine positive Wirkung der untersuchten Beschilderung auf die Verkehrssicherheit erwartet werden.

Insbesondere die $\mathrm{v}_{85}$ konnte durch die gelbe Beschilderung gemindert werden. Dies führte u.a. zu einer geringeren Bandbreite der Geschwindigkeiten (Homogenität). Sowohl die schnelleren Fahrzeuge, als auch die Bandbreite der Geschwindigkeiten sind aufgrund des gleichgerichteten Verkehrs, wie er auf Autobahnen und deren Baustellen vorliegt, von hoher Sicherheitsrelevanz.

Aufgrund des kurzen Beobachtungszeitraums ließen sich keine Aussagen zum Unfallgeschehen treffen. Hier sind weitere Untersuchungen über längere Zeiträume erforderlich.

Die Wirkung der eingesetzten Pfeilbaken auf die Erkennbarkeit und Leitwirkung in den Bereichen der Fahrstreifenverschwenkung war nicht Gegenstand der Untersuchung. Deren Einsatz erfolgte aufgrund der positiven Ergebnisse der Untersuchungen von BAIER et al. (2006) und SCHULZE et al. (2010). Darin wird für die Bereiche von Fahrstreifenverschwenkungen und -überleitungen die Verwendung von Pfeilbaken empfohlen. Beide Untersuchungen haben gezeigt, dass diese die Erkennbarkeit und Begreifbarkeit von Verschwenkungsbereichen erhöhen können.

Eine Untersuchung der Langzeitwirkung der gelben Beschilderung auf das Fahrverhalten in Baustellenbereichen mit geringer Verkehrssicherheit konnte aufgrund des kurzen Untersuchungszeitraums nicht vorgenommen werden. Ebenso konnte nicht geklärt werden, ob sich die erzielten Effekte bei einem standardmäßigen Einsatz aufrechterhalten lassen. BAIER et al. (2006) vermuten bei regelmäßigem Einsatz selektivgelber Verkehrszeichen eine Verringerung der Wirkung. Außerdem besteht weiterer Forschungsbedarf zur Auswirkung der gelben Beschilderung auf das Unfallgeschehen. 
Aus diesen Ergebnissen lässt sich für den praktischen Einsatz Folgendes ableiten:

Der Einsatz gelber Beschilderung vor allem am Baustellenbeginn ist zu empfehlen. Sowohl im Bereich der Fahrstreifenreduzierung, als auch am Beginn der Fahrstreifenverschwenkung konnten durch das Hervorheben der Verkehrszeichen und -einrichtungen (Baken) Geschwindigkeitsreduzierungen erreicht werden. Es sollten aber nur die Verkehrszeichen hervorgehoben werden, die im direkten Zusammenhang mit der bevorstehenden Situation, wie der Fahrstreifenverschwenkung oder -reduzierung stehen. Darüber hinaus sind, wie schon bei BAIER et. al. (2006) beschrieben, inkonsistente Gestaltungen (Vermischung gelber und weißer Verkehrszeichen) bei der Verwendung gelber Beschilderung zu vermeiden. Ebenso sollte die gelbe Beschilderung nur an wichtigen Stellen zum Einsatz kommen, da ein Verschleiß der Wirkung zu vermuten ist. Dies sind solche Baustellenbereiche, die sich zum Beispiel in QSV (2010) als besonders verkehrsunsicher herausgestellt haben.

Am Baustellenbeginn sollten in den Annäherungsbereichen alle Fahrstreifen- bzw. Überleitungstafeln und Baken mit selektivgelber Hintergrundfolie gestaltet werden, wenn:

- am Baustellenbeginn eine Fahrstreifenverschwenkung erfolgt.

- am Baustellenbeginn die Fahrstreifen in einer Überleitung mit einem Verschwenkmaß über 1:25ㄴ verschwenkt werden.

- im Zuge der Fahrstreifenüberleitung ein starker Querneigungswechsel aufgrund einer ausgeprägten gegenläufigen Querneigung im Mittelstreifen (RSA, 95) stattfindet.

Zudem sollten die mit der Fahrstreifenverschwenkung bzw. -überleitung im Zusammenhang stehenden Geschwindigkeitsbegrenzungen durch die Verwendung einer selektivgelben Hintergrundtafel hervorgehoben werden.

Vor Verschwenkungen oder Fahrstreifenüberleitungen am Baustellenende sollten gelbe Fahrstreifen- und Überleitungstafeln vorgesehen werden, wenn:

- Fahrstreifenüberleitungen ein Verschwenkmaß über 1:25 aufweisen

- Fahrstreifenverschwenkungen erfolgen.
Auf Bereiche von Fahrstreifenverschwenkungen am Baustellenende wird meist nicht mit Beschilderungen hingewiesen. Gleichzeitig wurde in QSV (2010) für diese Bereiche die höchste Unfallraten für Fahrstreifenverschwenkungen und Überleitungen bestimmt. Aus diesem Grund und auf Basis der Ergebnisse für Überleitungen am Baustellenende, sollten auch $200 \mathrm{~m}$ vor Verschwenkungen am Baustellende gelbe Fahrstreifentafeln angeordnet werden.

Neben den Bereichen am Beginn und Ende der Baustelle sollten die Fahrer auch in den Zufahrten von Anschlussstellen in Baustellenbereichen auf die bevorstehende Situation hingewiesen werden, wenn nur ein verkürzter Beschleunigungsstreifen zur Verfügung steht. Dazu sollte das Zeichen 123 in der Zufahrtsrampe durch eine gelbe Hintergrundtafel hervorgehoben werden.

Grundsätzlich ist bei der Anordnung gelber Beschilderung eine Vermischung gelber und weißer Verkehrszeichen zu vermeiden.

Die vorgenommene stufenweise Anhebung nach der Baustelle führte zu einer geringeren Bandbreite und damit zu einer größeren Homogenität der Geschwindigkeiten. Dies ist insbesondere auf Autobahnen ein wichtiges Kriterium der Verkehrssicherheit. Eine stufenweise Anhebung der zulässigen Höchstgeschwindigkeit in Schritten von $30 \mathrm{~km} / \mathrm{h}$ und Abständen von mindestens $500 \mathrm{~m}$ nach Baustellenende ist demnach zu empfehlen. Dabei sollte die erste Beschränkung nach der Baustelle $100 \mathrm{~km} / \mathrm{h}$ betragen.

Zudem ist unabhängig von der Verwendung gelber Beschilderung der Einsatz von Pfeilbaken, die in verschiedenen Untersuchungen gegenüber Schraffenbaken eine bessere Leitwirkung aufwiesen, zu empfehlen.

Die praktische Umsetzung dieser Empfehlungen in breitem Umfang setzt allerdings die Schaffung entsprechender Voraussetzungen in VwV-StVO und RSA voraus.

4 Nach RSA (RSA, 1995) wird für die Führung der Verschwenkungen ein Verhältnis von 1:20 empfohlen. Das bedeutet, dass auf 20 Meter Länge die Fahrstreifen um einen Meter seitwärts verschwenkt werden. Das hier angegebene Maß für die Überleitungen von 1:25 hat sich in ENKE et. al (2010) als unsicherste Gestaltungsform für Überleitungen herausgestellt. Dabei sind bei diesem Maß geringere Kurvenradien erforderlich. 


\section{Literaturverzeichnis}

Baier, M.; Kemper, D.; Baur, O.; Steinauer, B.; Frank, H.: Sicherheitswirkung von fluoreszierenden Materialien bei Leiteinrichtungen in Arbeitsstellen. Bundesministerium für Verkehr, Bonn 2006

Becker, Hubert und Schmuck: Verkehrsablauf an Autobahnbaustellen. Hochschule der Bundeswehr, München 1983.

Brühning, E.; Ernst, R.: Untersuchungen über den Verkehrsablauf und die zulässige Geschwindigkeit auf den Behelfsfahrstreifen im Bereich der Reparaturbaustellen der Bundesautobahn (Ergänzender Bericht). Bundesanstalt für Straßenwesen (BASt), Köln 1971

Brühning, E. Untersuchung der Unfälle mit Personenschaden auf Autobahnen. Forschung Straßenbau und Straßenverkehrstechnik; 223. 1977

Emde, W.; Hamester, H.: Unfallgeschehen an Autobahnbaustellen. Hochschule der Bundeswehr, München 1983.

Enke, M.; Lippold, C.; Maier, R.; Schulz, R.: Untersuchung der Verkehrssicherheit im Bereich von Baustellen auf Bundesautobahnen. Untersuchung im Auftrag Unfallforschung der Versicherer des GDV. Unveröffentlichter Bericht; Dresden, 2010

Merkblatt für Rahmenbedingungen für erforderliche Fachkenntnisse zur Verkehrssicherung von Arbeitsstellen an Straßen (MVAS 99). Forschungsgesellschaft für Straßen- und Verkehrswesen, Köln 1999

Merkblatt zum Auswerten von Straßenverkehrsunfällen - Teil 1: Führen und Auswerten von Unfalltypensteckkarten. Forschungsgesellschaft für Straßen- und Verkehrswesen, Köln 2003 (a)

Merkblatt zum Auswerten von Straßenverkehrsunfällen - Teil 2: Maßnahmen gegen Unfallhäufungen. Forschungsgesellschaft für Straßen- und Verkehrswesen, Köln 2003 (b)

Heller, F.; Lapierre, R.: Maßnahmen zur Aufrechterhaltung des Verkehrsflusses bei Bauarbeiten auf Bundesautobahnen. Zeitschrift für Verkehrssicherheit Nr. 11. 1965,

Kayser H..; Heß, M.; Feldges, M.; Struif, R.: Forschung Straßenbau und Straßenverkehrstechnik Entwicklung eines Instrumentariums zur Erstellung von Verkehrszeichenplänen und deren Umsetzung bei der Sicherung von Arbeitsstellen an Straßen. Bundesministerium für Verkehr, Bonn 1991.

Laube, M.: Verkehrsverhalten und Unfallgeschehen im Bereich von Autobahnbaustellen. ETH Zürich, Zürich 2001.

Lenz, K.-H.; Ernst, R.; Steinhoff, H.: Untersuchungen über den Verkehrsablauf und die zulässige Geschwindigkeit auf den Behelfsfahrstreifen im Bereich der Reparaturbaustellen der Bundesautobahn (Vorläufiger Schlussbericht) Bundesanstalt für Straßenwesen (BASt), Köln 1970.

Müller, F.; Seliger, R.: Untersuchung zur Wirkung unterschiedlicher Leiteinrichtungen als Fahrbahnverengung auf das Fahrverhalten vor BAB-Arbeitsstellen. In: Straße + Autobahn 41 (1990), Heft 10, S.452 - 458 
Pöppel-Decker, M.: Grundlagen streckenbezogener Unfallanalysen auf Bundesautobahnen. Bundesanstalt für StraBenwesen (BASt), M 153. Bergisch Gladbach 2003

FGSV.: Richtlinien für die Anlage von Straßen (RAS), Teil: Wirtschaftlichkeitsuntersuchungen (RAS - W). Forschungsgesellschaft für Straßen- und Verkehrswesen, Köln 1986.

Richtlinien für die Sicherung von Arbeitsstellen an Straßen (RSA-95). Bundesministerium für Verkehr, Bonn 1995.

Schüller, H.: Geschwindigkeitsverhalten in Stadtstraßen. Dissertation, Lehrstuhl Straßenverkehrstechnik mit Fachbereich Theorie der Verkehrsplanung, Fakultät Verkehrswissenschaften „Friedrich List“, Institut für Verkehrsplanung und Straßenverkehr, Dresden 2010

Straßenverkehrsordnung (StVO). Bundesministerium für Verkehr, Bonn 2007.

Allgemeine Verwaltungsvorschrift zur Straßenverkehrs-Ordnung (VwV-StVO). Bundesministerium für Verkehr, Bonn 2001. 


\section{Anhang}

\section{A Baustellenfreier Autobahnabschnitt}

\section{A.1 Freie Geschwindigkeiten auf dem baustellen- freien Autobahnabschnitt}

\section{Definition der frei-fahrenden Fahrzeuge}

Fahrzeuge die auf ein und demselben Fahrstreifen in die gleiche Richtung fahren können sich gegenseitig in ihrem Fahrverhalten beeinflussen. Insbesondere wenn die Längsabstände ein bestimmtes Maß unterschreiten wird das jeweilige Folgefahrzeug seine Geschwindigkeit dem Vorausfahrenden anpassen. In Schüller (2010) ${ }^{1}$ wurde das freie Fahren von Fahrzeugen über den zeitlichen Abstand zum jeweils vorausfahrenden Fahrzeug bestimmt. Unter Verwendung von Signifikanztests wurde der Zusammenhang von Abstand und Geschwindigkeit untersucht. Im Ergebnis zeigte sich, dass ab einer Lücke von mindestens 5 Sekunden nicht mehr von einer Beeinflussung des betrachteten Fahrzeugs durch den Vordermann zu rechnen ist. Da diese Untersuchung aber Innerortsstraßen zum Gegenstand hatte, wurde eine entsprechende Überprüfung auch für den Verkehrsablauf auf dem Untersuchungsabschnitt vorgenommen.

\section{Stichprobenhafte Überprüfung}

Grundlage für die Abschätzung der Kriterien für frei fahrende Fahrzeuge waren die Daten aus einer vierstündigen Videobeobachtung. Dabei wurden alle Fahrzeuge dieses Zeitraums erfasst und anschließend mit dem Videodetektionssystem RackVision ausgewertet. Die Untersuchung wurde für die Fahrzeuge, welche den rechten Fahrstreifenbefuhren, durchgeführt. Insgesamt wurden 5.059 Fahrzeuge erfasst und in Abhängigkeit ihrer Abstände zu vorausfahrenden und folgenden Fahrzeugen ausgewertet. Folgende Klassen wurden betrachtet:

\begin{tabular}{cc|c} 
& \multicolumn{2}{c}{ Abstand zum } \\
& $\begin{array}{c}\text { vorausfahrenden } \\
\text { Fahrzeug }\end{array}$ & $\begin{array}{c}\text { folgenden } \\
\text { Fahrzeug }\end{array}$ \\
\hline Weniger als & $5 \mathrm{~s}$ & $1 \mathrm{~s}$ \\
\hline mindestens & $5 \mathrm{~s}$ & $1 \mathrm{~s}$ \\
& $6 \mathrm{~s}$ & $2 \mathrm{~s}$ \\
& $7 \mathrm{~s}$ & $3 \mathrm{~s}$ \\
& $8 \mathrm{~s}$ & $4 \mathrm{~s}$ \\
& $9 \mathrm{~s}$ & $5 \mathrm{~s}$
\end{tabular}

Aus den Kombinationen der Zeitlücken vor und nach dem betrachteten Fahrzeug ergaben sich die in Tabelle A-1 dargestellten Fahrzeugzahlen, die Grundlage für die Auswertung mittlerer Geschwindigkeiten (Tabelle A-2) und im Weiteren für den Vergleich der Mittelwerte durch einen t-Test waren.

Für die Überprüfung, ab welchen Abständen andere Fahrzeuge die Geschwindigkeitswahl nicht mehr beeinflussen, wurden alle Zeitlückenkombinationen durch t-Tests miteinander verglichen. Beispielhaft sind in Tabelle A-3 und Tabelle A-4 die ermittelten t-Werte dargestellt. Dabei enthält Tabelle A-3 ausschließlich die Kombinationen, welche kurze Zeitlücken entweder zum vorausfahrenden (unter $5 \mathrm{~s}$ ) oder folgenden Fahrzeug (unter $1 \mathrm{~s}$ ) beinhalten. Aus den grau eingefärbten Werten geht hervor, welche Kollektive sich signifikant voneinander unterscheiden. Mehr als die Hälfte der gegenübergestellten Kollektive weist nachweisbar unterschiedliche Geschwindigkeiten auf. Vergleicht man dagegen Zeitlückenkombinationen, die zum vorausfahrenden Fahrzeug mindestens $5 \mathrm{~s}$ und zum Folgefahrzeug mindestens $1 \mathrm{~s}$ Abstand einhielten, können kaum noch bzw. nur geringe Unterschiede zwi-

1 Schüller, H.: Modelle zur Beschreibung des Geschwindigkeitsverhaltens auf Stadtstraßen und dessen Auswirkungen auf die Verkehrssicherheit auf Grundlage der Straßengestaltung. Dissertation. Fakultät Verkehrswissenschaften „Friedrich List“; Institut für Verkehrsplanung und Straßenverkehr; Professur für Straßenverkehrstechnik, Technische Universität Dresden; Dresden, 2010 
Tabelle A-1:

Anzahlen frei fahrender Fahrzeuge in Abhängigkeit der Zeitlücken zum vorausfahrenden und folgenden Fahrzeug auf dem rechten Fahrstreifen

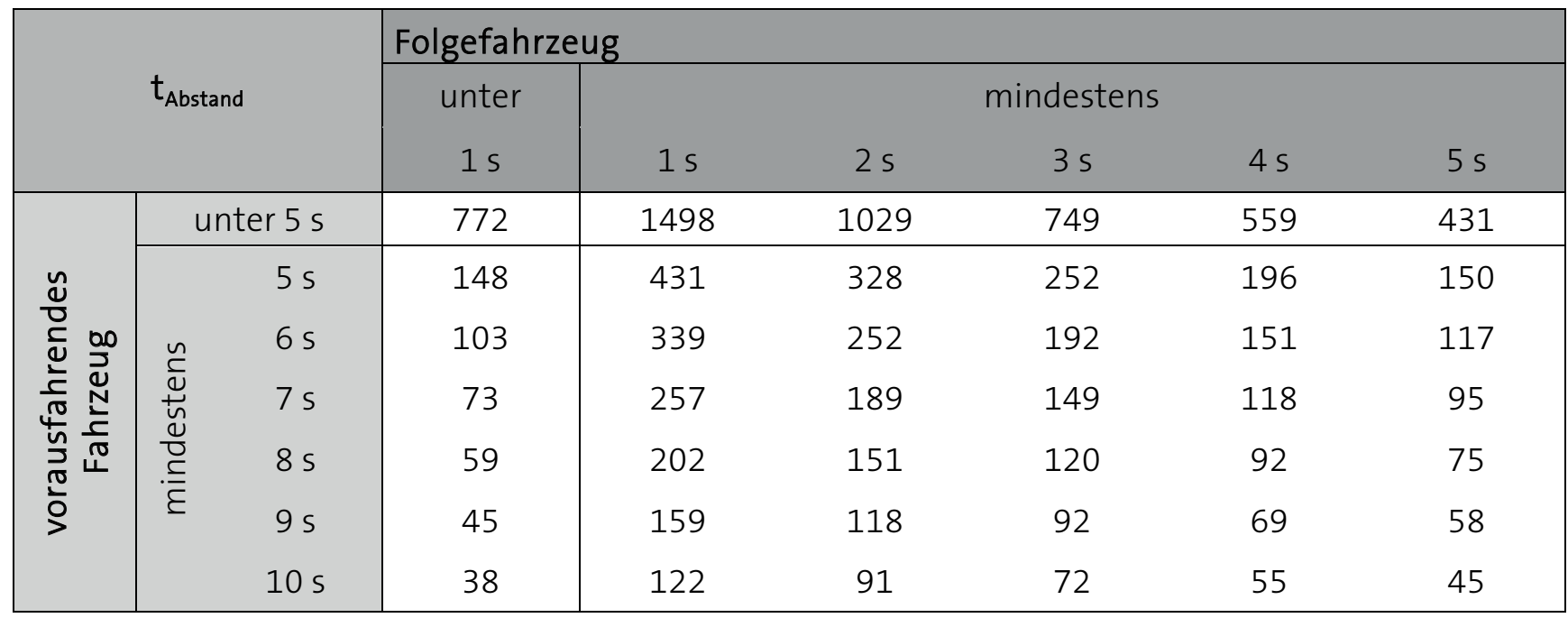

Tabelle A-2:

mittlere Geschwindigkeiten von Fahrzeugkollektiven in Abhängigkeit der Zeitlücken zum vorausfahrenden und folgenden Fahrzeug auf dem rechten Fahrstreifen

\begin{tabular}{|c|c|c|c|c|c|c|c|c|}
\hline \multirow{2}{*}{\multicolumn{3}{|c|}{$t_{\text {Abstand }}$}} & \multicolumn{6}{|c|}{ Folgefahrzeug } \\
\hline & & & \multirow{2}{*}{$\begin{array}{l}\text { unter } \\
1 \mathrm{~s}\end{array}$} & \multicolumn{5}{|c|}{ mindestens } \\
\hline & & & & $1 \mathrm{~s}$ & $2 \mathrm{~s}$ & $3 s$ & $4 s$ & $5 \mathrm{~s}$ \\
\hline \multirow{7}{*}{ 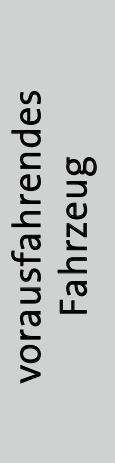 } & & nter $5 \mathrm{~s}$ & 108,2 & 114,5 & 115,9 & 116,3 & 117,4 & 117,0 \\
\hline & \multirow{6}{*}{ 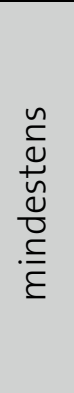 } & $5 \mathrm{~s}$ & 112,2 & 124,9 & 126,1 & 127,1 & 127,7 & 128,0 \\
\hline & & $6 s$ & 111,0 & 124,6 & 125,3 & 126,5 & 126,7 & 128,1 \\
\hline & & $7 s$ & 110,4 & 125,6 & 126,3 & 127,6 & 127,1 & 128,2 \\
\hline & & $8 s$ & 111,0 & 128,4 & 129,1 & 130,2 & 130,3 & 130,7 \\
\hline & & $9 \mathrm{~s}$ & 111,7 & 127,9 & 128,3 & 129,0 & 129,7 & 130,2 \\
\hline & & $10 \mathrm{~s}$ & 110,8 & 125,8 & 124,9 & 123,9 & 125,1 & 123,9 \\
\hline
\end{tabular}

schen den Geschwindigkeiten der Kollektive nachgewiesen werden. Das wird u.a. aus Tabelle A-4 deutlich. Alle Gegenüberstellungen bei denen die Abstände mindestens 5 s zum Vordermann und 1 s zum Folgefahrzeug aufwiesen weisen keine signifikanten Unterschiede zwischen den gemessenen Geschwindigkeiten auf. Einzige Ausnahme ist die Gegenüberstellung der Kollektive (Zeitlücke voraus / Zeitlücke zurück) $5 \mathrm{~s} / 4 \mathrm{~s}$ und $6 \mathrm{~s} / 1 \mathrm{~s}$.
Auf Basis der Ergebnisse werden Fahrzeuge als durch andere Verkehrsteilnehmer unbeeinflusst fahrend angenommen, wenn sie zum vorausfahrenden Fahrzeug mindestens $5 \mathrm{~s}$ und zum folgenden Fahrzeug $2 \mathrm{~s}$ Abstand aufweisen. Das entspricht bei einer Geschwindigkeit von $120 \mathrm{~km} / \mathrm{h}$ einer Nettoraumlücke von fast $170 \mathrm{~m}$ zum Vordermann und fast $70 \mathrm{~m}$ zum Folgefahrzeug. 
Tabelle A-3:

t-Werte für Vergleich der mittleren Geschwindigkeiten von Fahrzeugkollektiven unterschiedlicher Abstände zu vorausfahrenden und folgenden Fahrzeugen

\begin{tabular}{|c|c|c|c|c|c|c|c|c|}
\hline \multirow{3}{*}{\multicolumn{2}{|c|}{$\begin{array}{l}\text { voraus- } \\
\text { fahrendes } \\
\text { Fahrzeug }\end{array}$}} & & \multicolumn{6}{|c|}{ Abstand voraus unter $5 \mathrm{~s}$} \\
\hline & & \multirow{2}{*}{$\begin{array}{c}\text { Folge- } \\
\text { fahrzeug }\end{array}$} & \multirow{2}{*}{$\begin{array}{l}\text { unter } \\
1 \mathrm{~s}\end{array}$} & \multicolumn{5}{|c|}{ mindestens } \\
\hline & & & & $1 \mathrm{~s}$ & $2 \mathrm{~s}$ & $3 s$ & $4 s$ & $5 \mathrm{~s}$ \\
\hline \multicolumn{2}{|c|}{ unter $5 \mathrm{~s}$} & \multirow{7}{*}{ 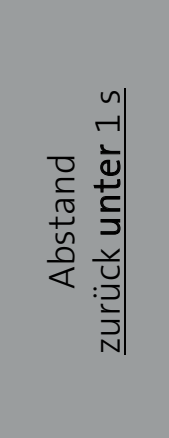 } & - & $-6,8$ & $-7,4$ & $-7,5$ & $-7,7$ & $-6,5$ \\
\hline \multirow{6}{*}{ 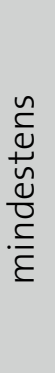 } & $5 s$ & & 2,0 & $-1,3$ & $-1,9$ & $-2,1$ & $-2,6$ & $-2,1$ \\
\hline & $6 s$ & & 1,2 & $-1,6$ & $-2,1$ & $-2,4$ & $-2,8$ & $-2,3$ \\
\hline & $7 \mathrm{~s}$ & & 0,8 & $-1,6$ & $-2,0$ & $-2,3$ & $-2,6$ & $-2,2$ \\
\hline & $8 s$ & & 1,0 & $-1,3$ & $-1,6$ & $-1,9$ & $-2,2$ & $-1,8$ \\
\hline & $9 \mathrm{~s}$ & & 1,1 & $-0,9$ & $-1,2$ & $-1,4$ & $-1,7$ & $-1,4$ \\
\hline & $10 \mathrm{~s}$ & & 0,7 & $-1,1$ & $-1,4$ & $-1,6$ & $-1,8$ & $-1,5$ \\
\hline
\end{tabular}

Tabelle A-4:

t-Werte für Vergleich der mittleren Geschwindigkeiten von Fahrzeugkollektiven unterschiedlicher Abstände zu vorausfahrenden und folgenden Fahrzeugen

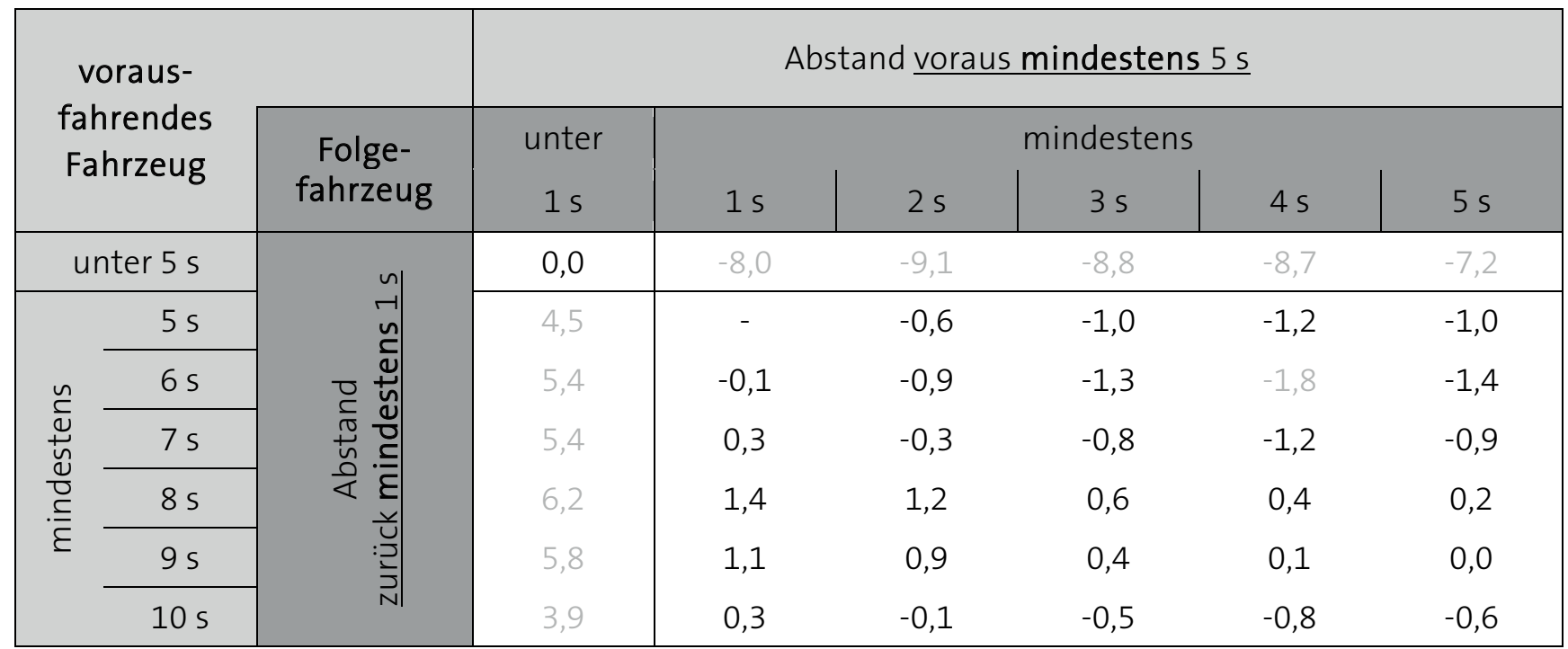




\section{A.2 Verkehrsablauf}

Außerhalb der Baustelle stehen den insgesamt 37.663 Fahrzeugen (Schwerverkehrsanteil 14,5 \%) in Fahrtrichtung 1 zwei und in Fahrtrichtung 2 drei Fahrstreifen zur Verfügung.

Im Rahmen einer Querschnittsmessung wurde an einem Wochentag in der drei-streifigen Fahrtrichtung 2 über einen Be-obachtungszeitraum von vier Stunden insgesamt 3.308 Pkw und 512 Lkw erfasst. In der zweistreifigen Gegenrichtung wurden 4.242 Pkw und 817 Lkw gezählt. Eine Auswertung der Fahrstreifenbelegung in Abhängigkeit von der Verkehrsstärke in dieser Fahrtrichtung ergab bereits bei geringen Verkehrsstärken einen hohen Belegungsanteil des Überholfahrstreifens. Bei ca. $1.600 \mathrm{Fz} / \mathrm{h}$ fahren ca. 50 \% aller Fahrzeuge auf dem linken Fahrstreifen (Abbildung A-1). Bei einer Auswertung des gesamten Untersuchungszeitraums in 5 min-Intervallen ergab sich für den rechten Fahrstreifen eine maximale Verkehrsstärke von etwa $900 \mathrm{Fz} / \mathrm{h}$ und für den Überholfahrstreifen von fast $1.050 \mathrm{Fz} / \mathrm{h}$. Dies kann u.a. durch den geringeren Platzbedarf von Pkw, die überwiegend den linken Fahrstreifen nutzten, gegenüber dem Schwerverkehr auf dem rechten Fahrstreifen erklärt werden.

Die Zeitlücken zwischen den aufeinanderfolgenden Fahrzeugen stehen in engen Zusammenhang mit den Verkehrsstärken der jeweiligen Fahrstreifen. Für eine $A b$ schätzung des Abstandsverhaltens außerhalb der zu untersuchenden Baustelle wurden die Längsabstände zum jeweils vorausfahrenden Fahrzeug für beide Fahrtrichtungen in 5 min-Intervallen und unterteilt in vier Klassen betrachtet:

- weniger als $1 \mathrm{~s}$

- zwischen $1 \mathrm{~s}$ und $2 \mathrm{~s}$

- zwischen $2 \mathrm{~s}$ und $3 \mathrm{~s}$

- über $3 \mathrm{~s}$.
Mit der Zunahme der Verkehrsstärken nimmt die Zahl der Zeitlücken über $3 \mathrm{~s}$ kontinuierlich ab. Gleichzeitig steigt der Anteil kürzerer und insbesondere der sehr kurzen (weniger als $1 \mathrm{~s}$ ) Abstände. Bei Verkehrsstärken zwischen $800 \mathrm{Fz} / \mathrm{h}$ und $900 \mathrm{Fz} / \mathrm{h}$ wiesen bis zu $50 \%$ aller Zeitlücken eine Dauer von weniger als $1 \mathrm{~s}$ auf. Eine ähnliche Tendenz ist auf dem Überholfahrstreifen zu beobachten (Abbildung A-2). Der Anteil kurzer Zeitlücken ist jedoch auf dem rechten Fahrstreifen bei vergleichbaren Verkehrsstärken höher als auf dem Überholfahrstreifen.

Für die Geschwindigkeitsanalyse auf dem untersuchten Autobahnabschnitt außerhalb der Baustelle wurden frei fahrende Fahrzeuge betrachtet. Zur Abgrenzung freier Fahrzeuge wurden die in Anhang A.1 beschriebenen Kriterien zu Grunde gelegt. In Anhang C sind die zugehörigen Analysen zu den Längsabständen sowohl zum vorausfahrenden, als auch zum folgenden Fahrzeug enthalten. Die mittlere Geschwindigkeit von Pkw betrug auf dem rechten Fahrstreifen $130 \mathrm{~km} / \mathrm{h}$ und auf dem linken Fahrstreifen etwa $150 \mathrm{~km} / \mathrm{h}$. Die v85 lagen in Fahrtrichtung 2 etwa $15 \mathrm{~km} / \mathrm{h}$ bis $20 \mathrm{~km} / \mathrm{h}$ und in Richtung 1 etwa $30 \mathrm{~km} / \mathrm{h}$ über den mittleren Geschwindigkeiten (Tabelle A-5). Die mittleren Geschwindigkeiten für Lkw betrugen zwischen $86 \mathrm{~km} / \mathrm{h}$ und $101 \mathrm{~km} / \mathrm{h}$.

Die für Lkw vergleichsweise ermittelten hohen v85 können nur ansatzweise mit dem Verkehrsablauf erklärt werden. Durch die automatisierte Auswertung mit dem System RackVision werden aber auch Transporter der Gruppe der Lkw zugeordnet. Außerdem können aufgrund der Kameraposition Verdeckungen von zwei dicht aufeinanderfolgenden Pkw zu Fehldetektionen führen. Aus diesem Grund werden die Ergebnisse in weiteren Arbeitsschritten mit den Daten von automatischen Zählstellen verglichen und bewertet. 


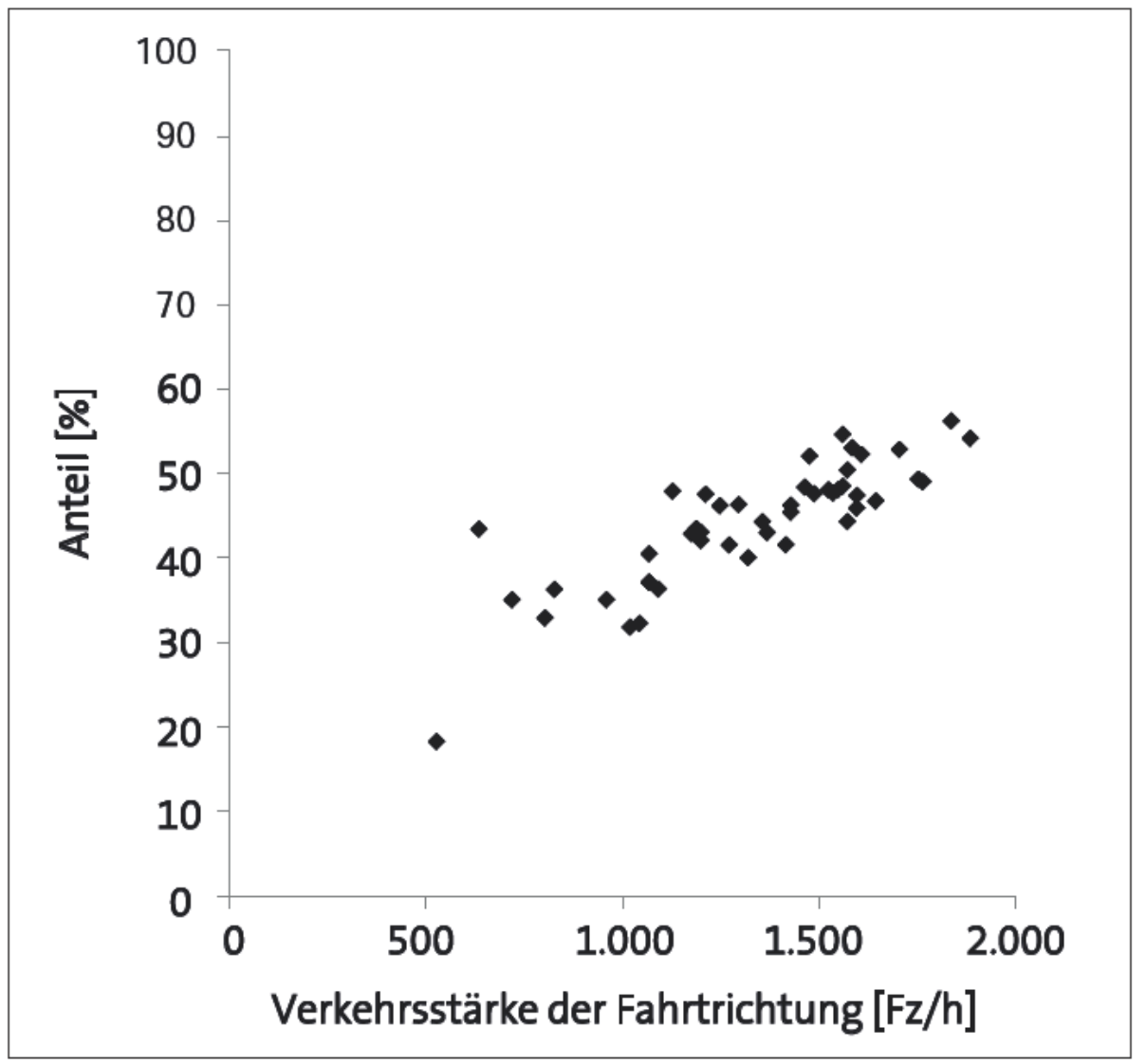

Abbildung A-1:

Belegung des linken Fahrstreifens (FS2) in Abhängigkeit von der Gesamtverkehrsstärke der Fahrtrichtung

Tabelle A-5:

Geschwindigkeitskenngrößen frei fahrender Fahrzeuge

\begin{tabular}{|l|c|c|c|c|c|c|c|c|c|c|}
\hline \multirow{2}{*}{} & \multicolumn{4}{|c|}{ Fahrtrichtung 1 } & \multicolumn{5}{c|}{ Fahrtrichtung 2 } \\
\cline { 2 - 11 } & \multicolumn{2}{|c|}{ Fahrstreifen 1 } & \multicolumn{2}{|c|}{ Fahrstreifen 2 } & \multicolumn{2}{|c|}{ Fahrstreifen 1 } & \multicolumn{2}{|c|}{ Fahrstreifen 2 } & \multicolumn{2}{c|}{ Fahrstreifen 3 } \\
& Pkw & Lkw & Pkw & Lkw & Pkw & Lkw & Pkw & Lkw & Pkw & Lkw \\
\hline Anzahl & 335 & 80 & 548 & 16 & 266 & 78 & 758 & 28 & 142 & 0 \\
\hline $\mathbf{V}_{\mathbf{1 5}}$ & 108 & 87 & 127 & 92 & 106 & 75 & 125 & 83 & 125 & - \\
$\mathbf{V}_{\mathbf{5 0}}$ & 130 & 92 & 146 & 101 & 128 & 86 & 140 & 91 & 145 & \\
$\mathbf{V}_{\mathbf{8 5}}$ & 159 & 99 & 174 & 112 & 144 & 101 & 156 & 114 & 160 & \\
\hline
\end{tabular}




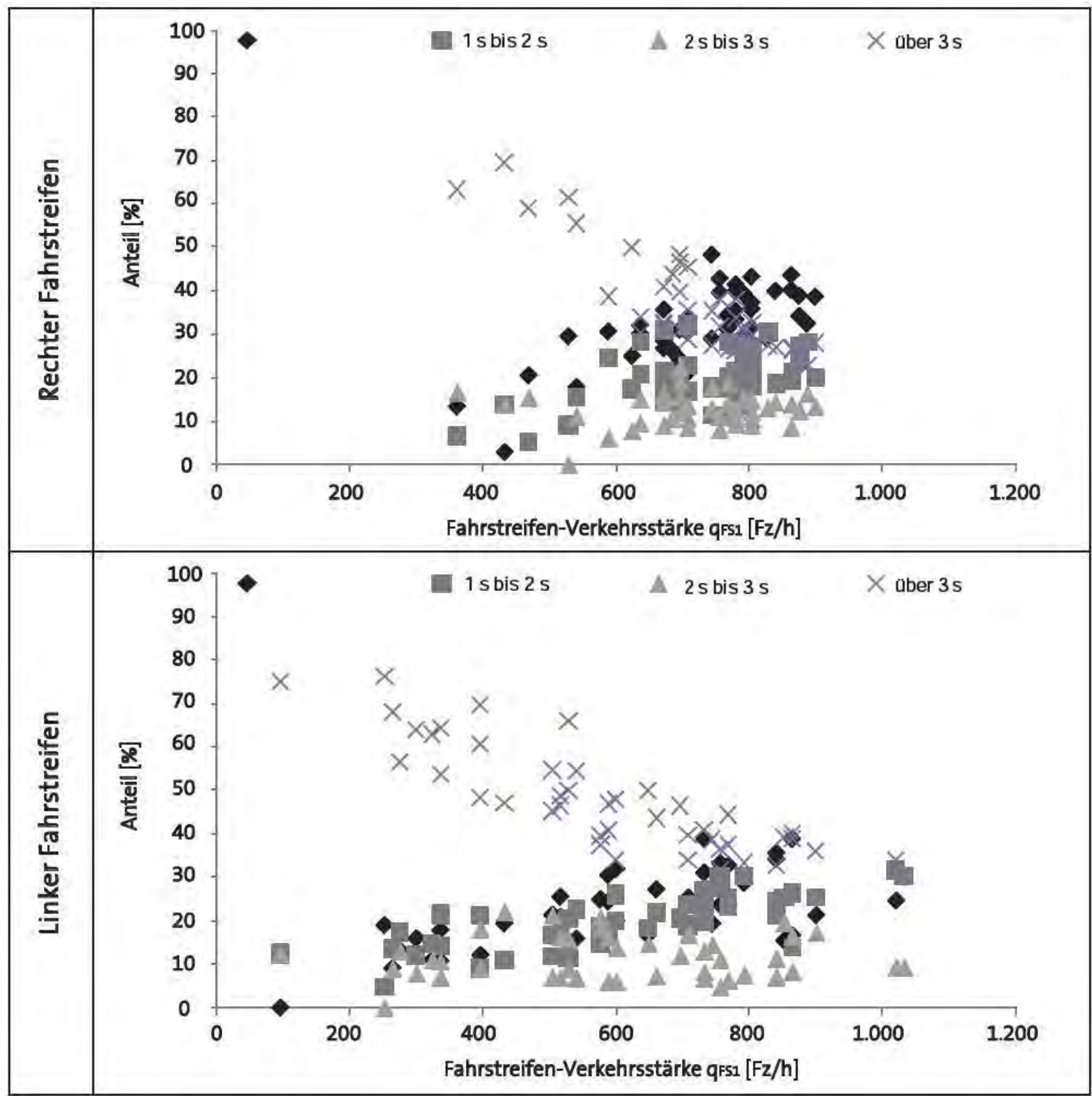

Abbildung A-2:

zeitlicher Längsabstand in Abhängigkeit der Fahrstreifen-Verkehrsstärke für Fahrtrichtung 1 


\section{B Untersuchungsbaustelle}

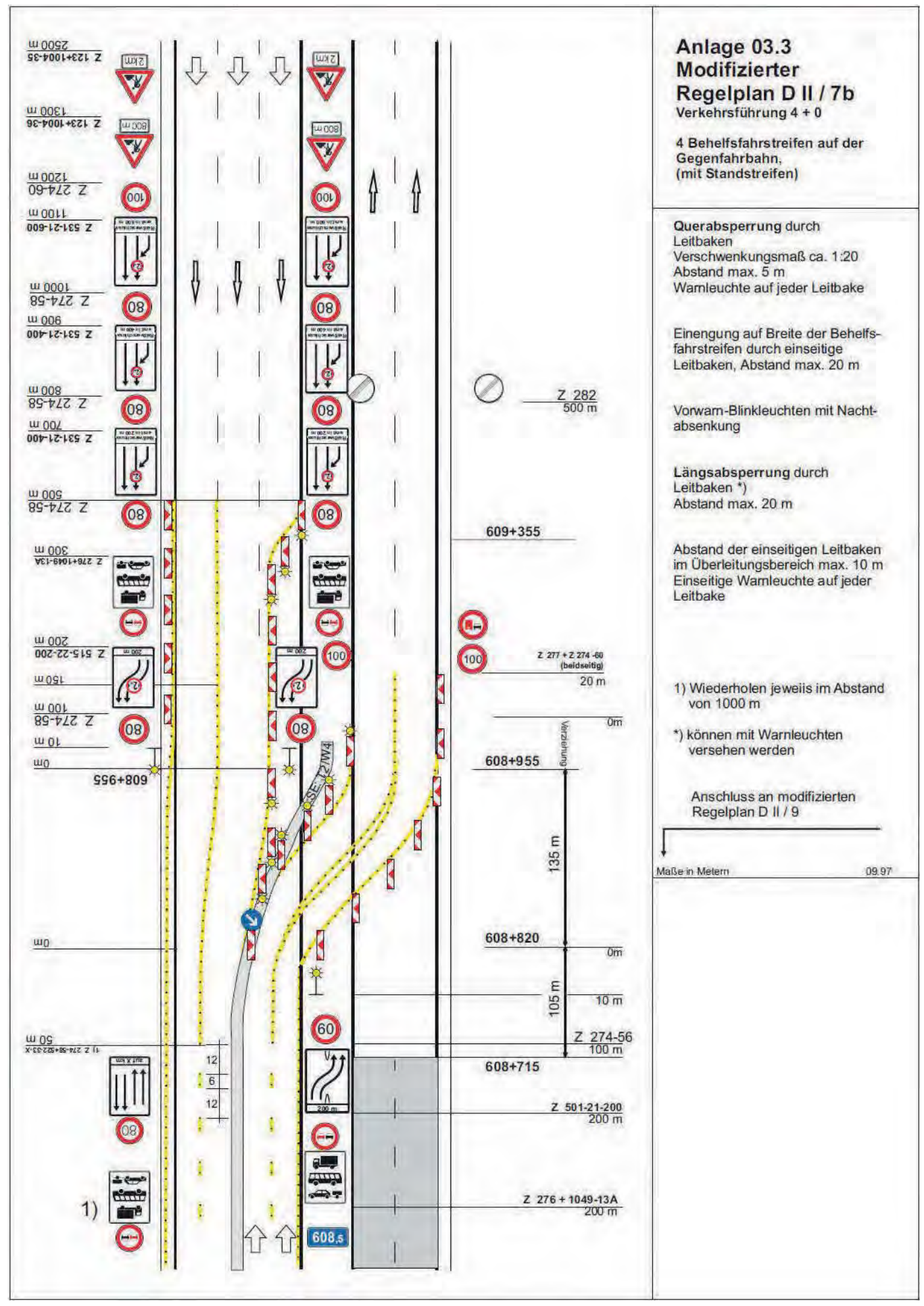

Abbildung B-1:

Regelplan Untersuchungsbaustelle mit Beschilderung nach RSA 95 - weiß - Teil 1 


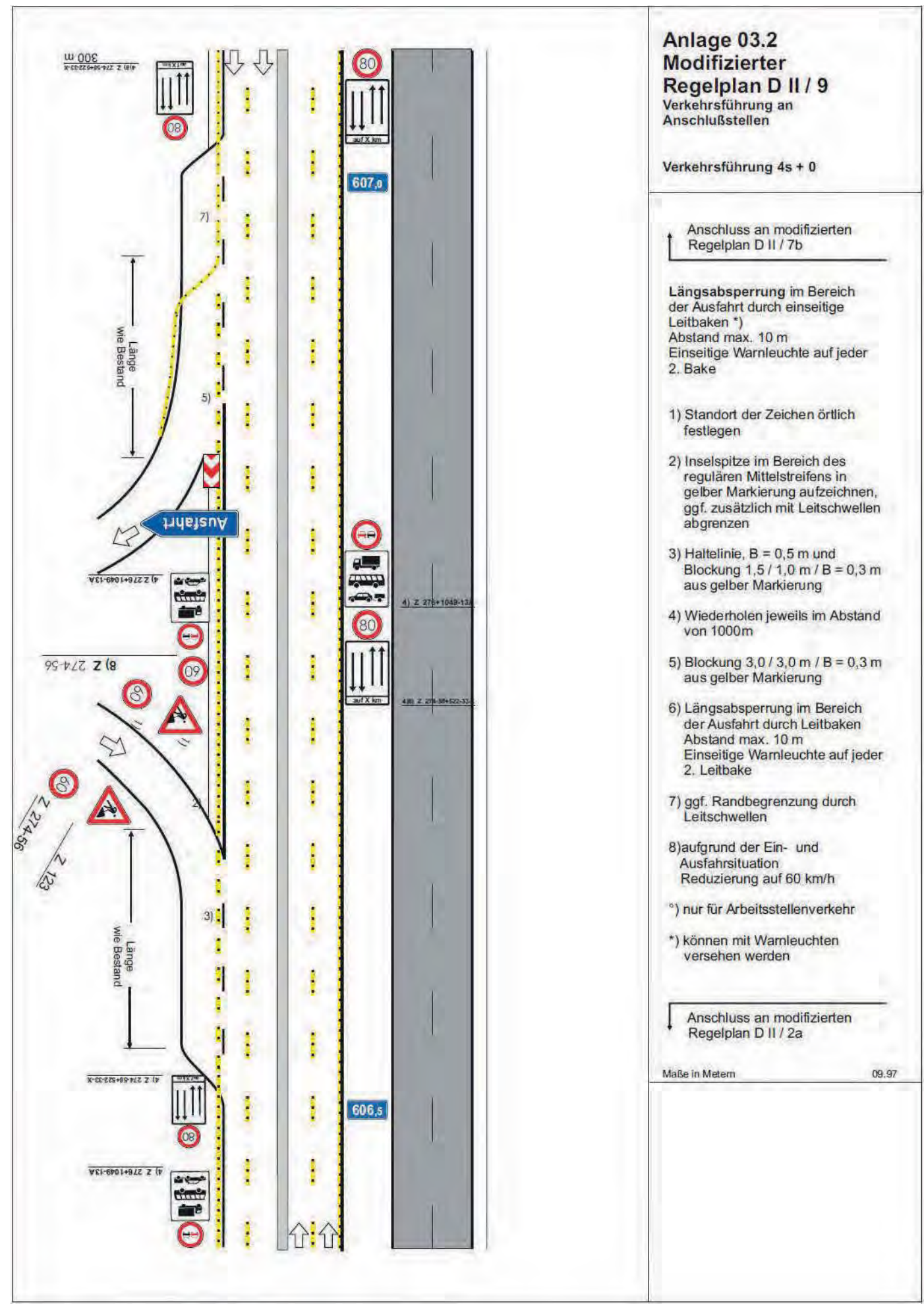

Abbildung B-2:

Regelplan Untersuchungsbaustelle mit Beschilderung nach RSA 95 - weiß - Teil 2 


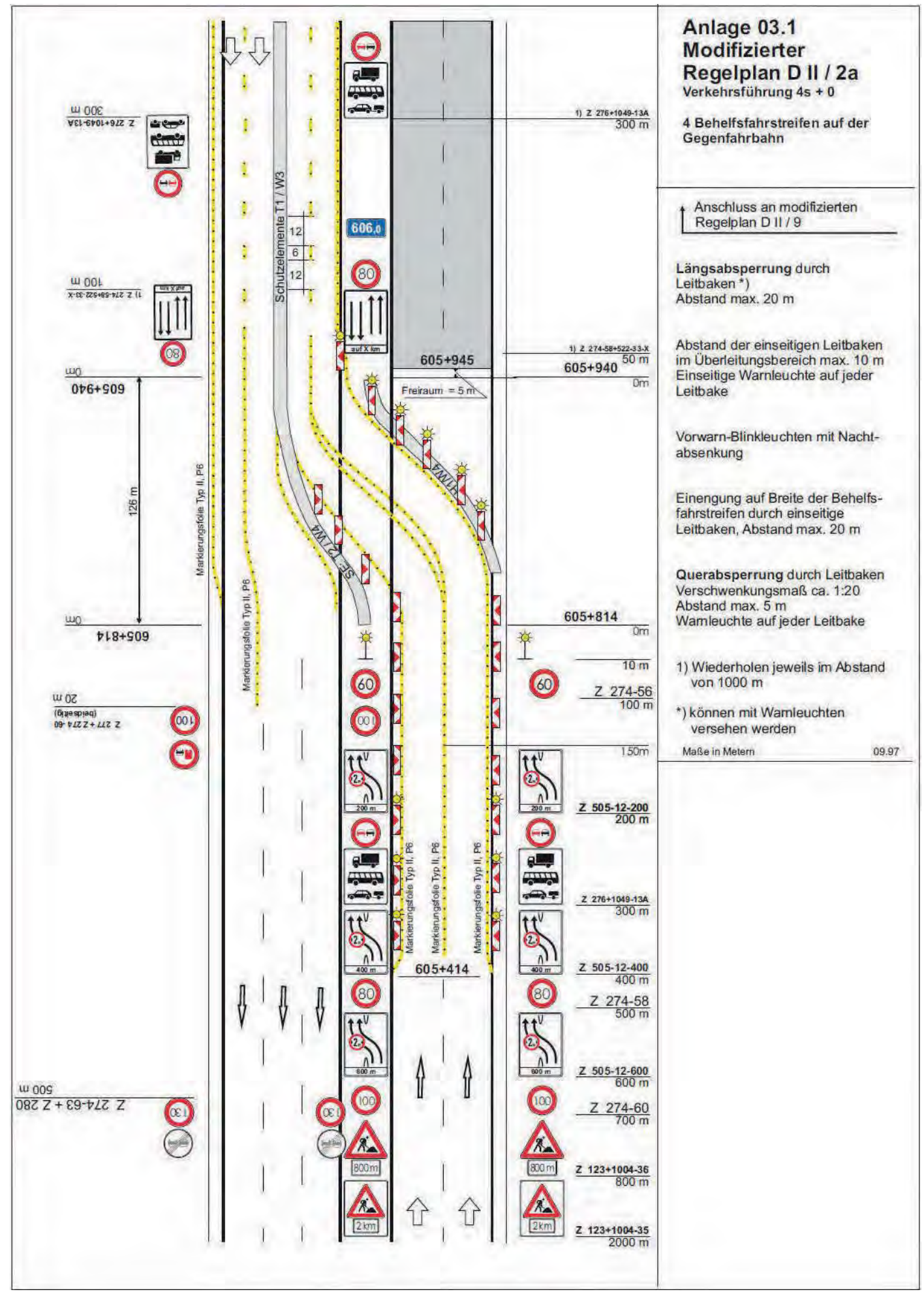

Abbildung B-3:

Regelplan Untersuchungsbaustelle mit Beschilderung nach RSA 95 - weiß - Teil 3 


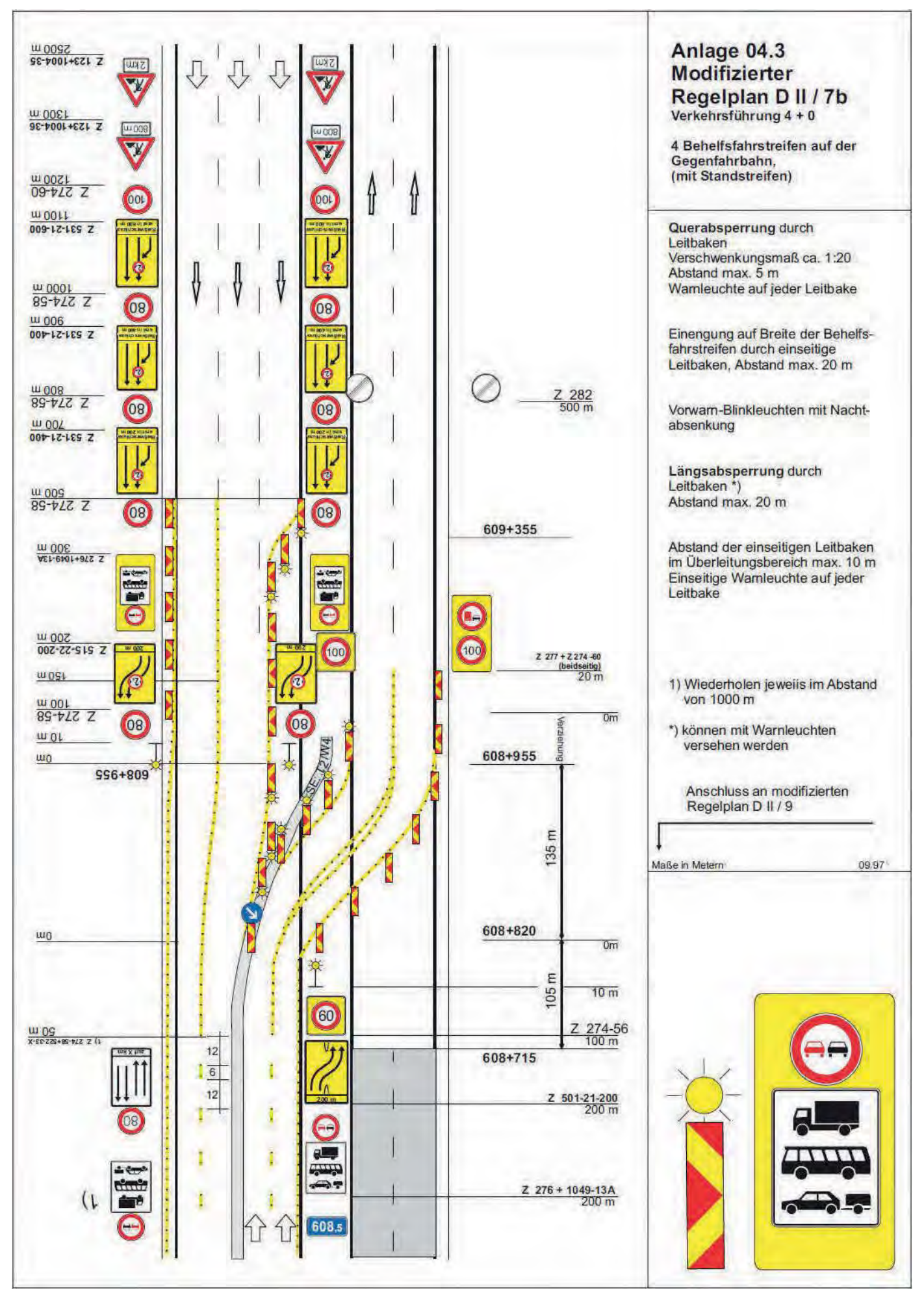

Abbildung B-4:

Regelplan Untersuchungsbaustelle mit Beschilderung nach RSA 95 - gelb - Teil 1 


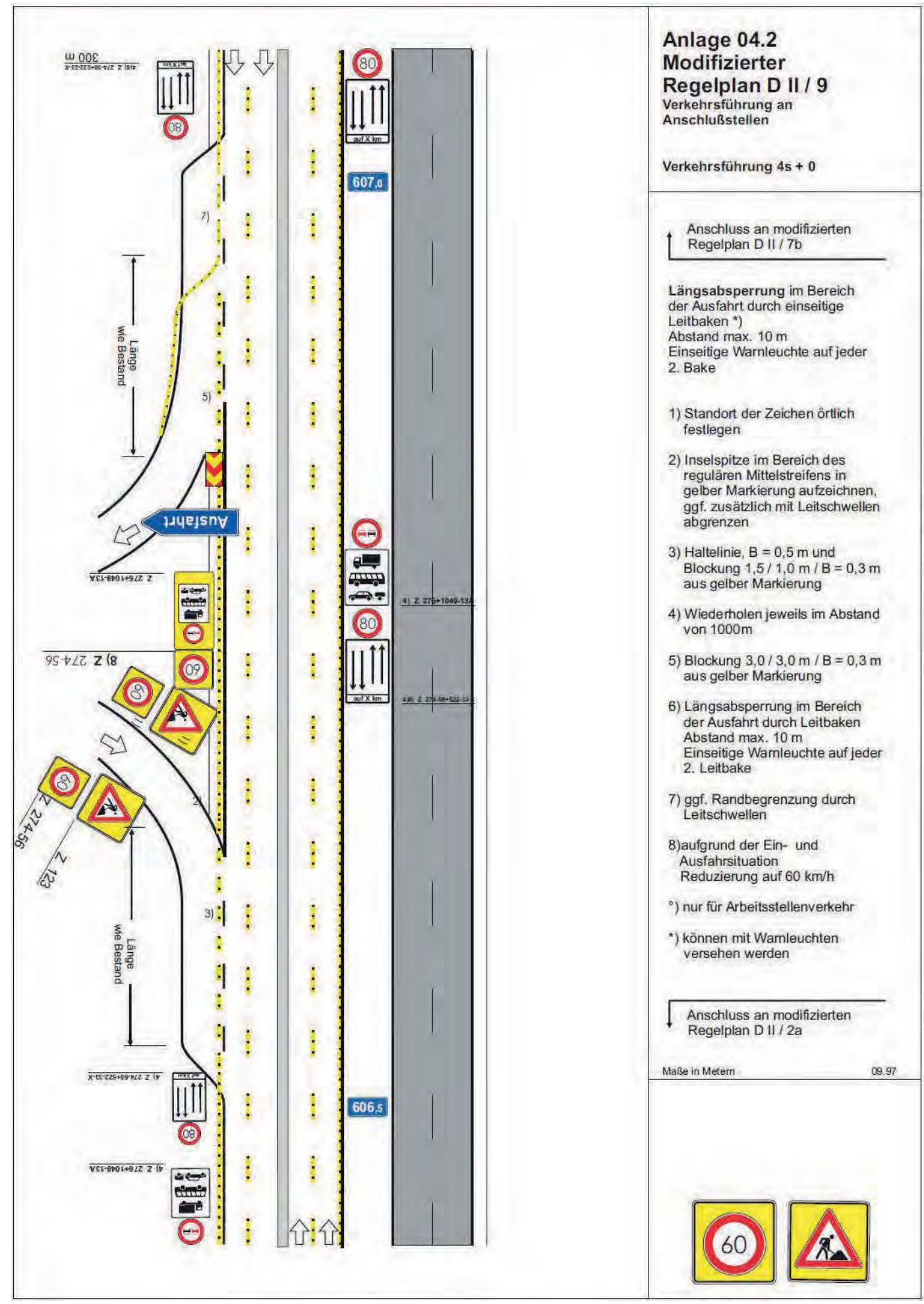

Abbildung B-5:

Regelplan Untersuchungsbaustelle mit Beschilderung nach RSA 95 - gelb - Teil 2 


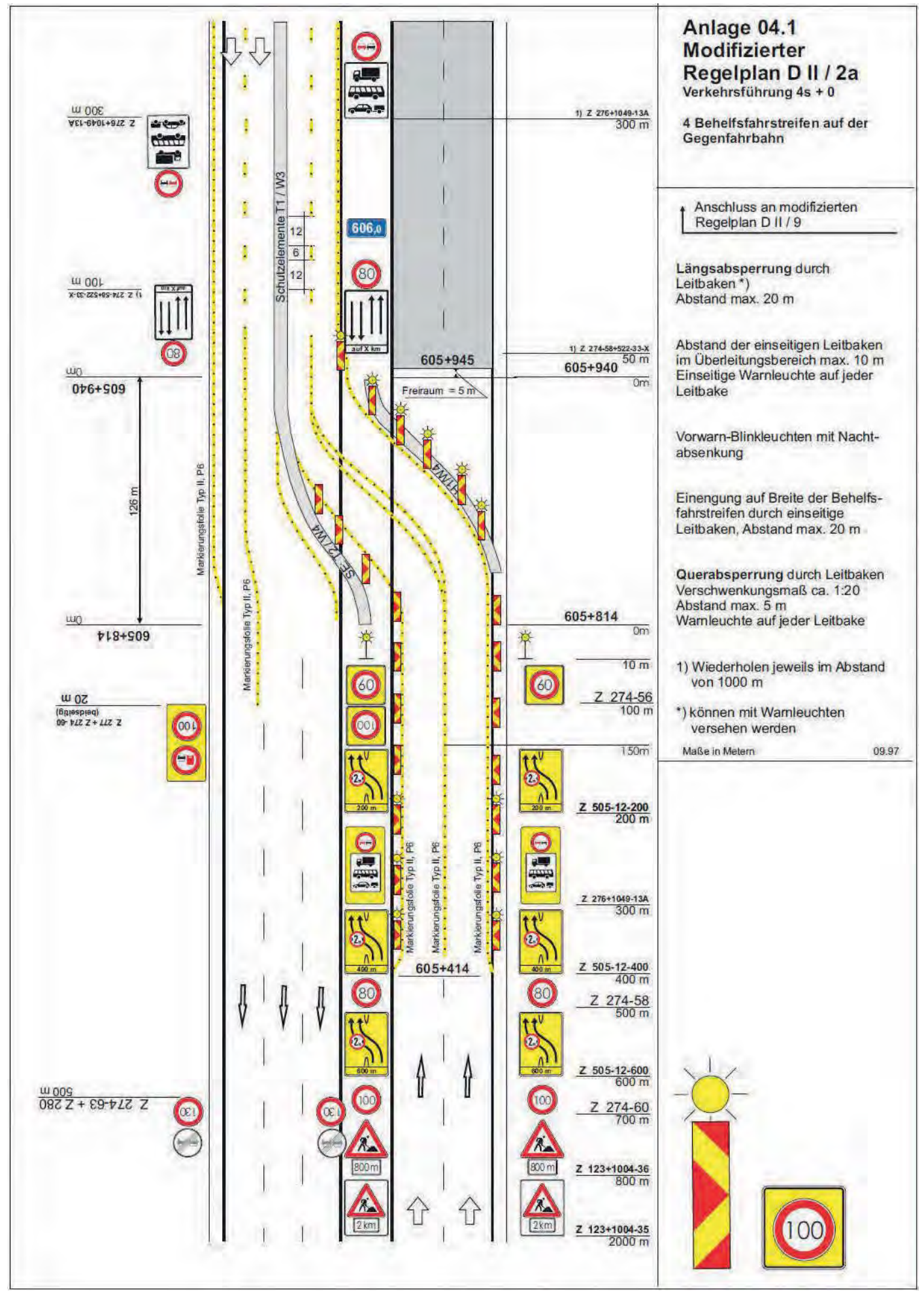

Abbildung B-6:

Regelplan Untersuchungsbaustelle mit Beschilderung nach RSA 95 - gelb - Teil 3 


\section{Liste der 3-stelligen Unfalltypen}

\section{C.1 Baustellenbereich}

Zunächst wurde für die Baustelle durch Nachfolgefahrten jeweils 60 Geschwindigkeitsprofiele im Vorherzeitraum und im Nachherzeitraum für jede Fahrtrichtung erstellt. Diese repräsentieren jeweils zur Hälfte verkehrsstarke und verkehrsschwache Zeiten.

In den Darstellungen der Verläufe sind die sich daraus ergebenden mittleren Geschwindigkeiten $v_{50}$ und die $v_{85}$ als maßgebliche Perzentilgeschwindigkeiten hervorgehoben. Die $v_{50}$ eignet sich für die allgemeine Beschreibung des Geschwindigkeitsverlaufs über die gesamte Baustelle. Für die Betrachtung der Einhaltung der zulässigen Höchstgeschwindigkeiten wird die $v_{85}$ verwendet. Die Differenz beider Werte gibt Auskunft über die Homogenität der gefahrenen Geschwindigkeiten.

Der Unterschied zwischen verkehrsstarken und verkehrsschwachen Zeiten zeigt sich deutlich in den Geschwindigkeitsprofilen, die beispielhaft in der Abbildung C-1 und Abbildung C-2 im Vorherzeitraum, in Richtung 1 dargestellt sind. Die Streuung der Geschwindigkeiten zu verkehrsschwachen Zeiten fällt deutlich größer aus als zu verkehrsstarken, die einen gleichmäßigen Verlauf aufweisen. In den Annäherungs- sowie in den Nachlaufbereichen traten gegenüber den Baustelleninnenbereichen größere Abweichungen zwischen $v_{85}$ und $v_{50}$ auf. $Z u$ verkehrsschwachen Zeiten lag die $v_{85}$ im Schnitt $20 \mathrm{~km} / \mathrm{h}$ über der zulässigen Höchstgeschwindigkeit. Diese wurde um bis zu 43 km/h im Baustelleninnebereich überschritten. Im Verschwenkungsbereich am Baustellenende traten bei einer zulässigen Höchstgeschwindigkeit von $60 \mathrm{~km} / \mathrm{h}$ Spitzenwerte von $118 \mathrm{~km} / \mathrm{h}$ auf.

Aus den Geschwindigkeitsprofilen der Verfolgungsfahrten konnten außerdem Aussagen zur Homogenität der Geschwindigkeit im Baustelleninnenbereich und zum allgemeinen Geschwindigkeitsniveau getroffen werden.
Dafür wurden die maßgebenden Perzentilgeschwindigkeiten $v_{85}$ und $v_{50}$ an verschiedenen Querschnitten des Innenbereichs der Baustelle herangezogen:

- Baustellenanfang (Verschwenkung/Überleitung)

- Anschlussstelle innerhalb der Baustelle

- Baustellenende (Rückverschwenkung)

Die Abbildung C-3 bis Abbildung C- 6 zeigen das Maß der Überschreitung der zulässigen Höchstgeschwindigkeit durch die $\mathrm{v}_{50}$ bzw. die $\mathrm{v}_{85}$. In diesen Abbildungen sind sowohl verkehrsschwache Zeiten und verkehrsstarke Zeiten als auch der Vorher-/Nachher-Vergleich dargestellt. Es wird deutlich, dass Überschreitungen der zulässigen Höchstgeschwindigkeit sowohl vor als auch nach der Einrichtung der selektivgelben Beschilderung der Regelfall sind. Dennoch ist eine Wirkung dieser Beschilderung vor allem in den Bereichen Baustellenanfang und Baustellenende zu verzeichnen. In verkehrsschwachen Zeiten sinkt die 50 in den Verschwenkungsbereichen um $3 \mathrm{~km} / \mathrm{h}$, die $\mathrm{v}_{85}$ sogar um 4-6 km/h. In verkehrsstarken Zeiten blieben die Perzentilgeschwindigkeiten zu Baustellenanfang und -ende unverändert.

Im Bereich der Anschlussstelle kann keine Tendenz zum Einfluss der selektivgelben Beschilderung abgelesen werden. Die $v_{50}$ steigt in verkehrsschwachen Zeiten im Vergleich zu den Vorhermessungen um $2 \mathrm{~km} / \mathrm{h}$ (Abbildung C-5) und die $v_{85}$ um 3 km/h (Abbildung C-6). Bei verkehrsstarken Zeiten ist hier jedoch ein anderes Bild zu erkennen. Während die $v_{50}$ nahezu unverändert bleibt (Differenz $1 \mathrm{~km} / \mathrm{h}$ ), geht die $v_{85}$ um $4 \mathrm{~km} / \mathrm{h}$ zurück.

Die Bewertung der Homogenität der Geschwindigkeiten erfolgte anhand der Differenz der $v_{85} z_{u r} v_{50}$. Die Differenzen werden in Abbildung C-7 und Abbildung C-8 gezeigt. Es ist zu erkennen, dass sowohl in Richtung 1 als auch in Richtung 2 zum Baustellenanfang die Geschwindigkeitsdifferenz abnimmt. Somit ist bei selektivgelber Beschilderung der Geschwindigkeitsverlauf in diesem Bereich homogener. In den anderen kritischen Bereichen kann kein signifikanter Unterschied festgestellt werden. 


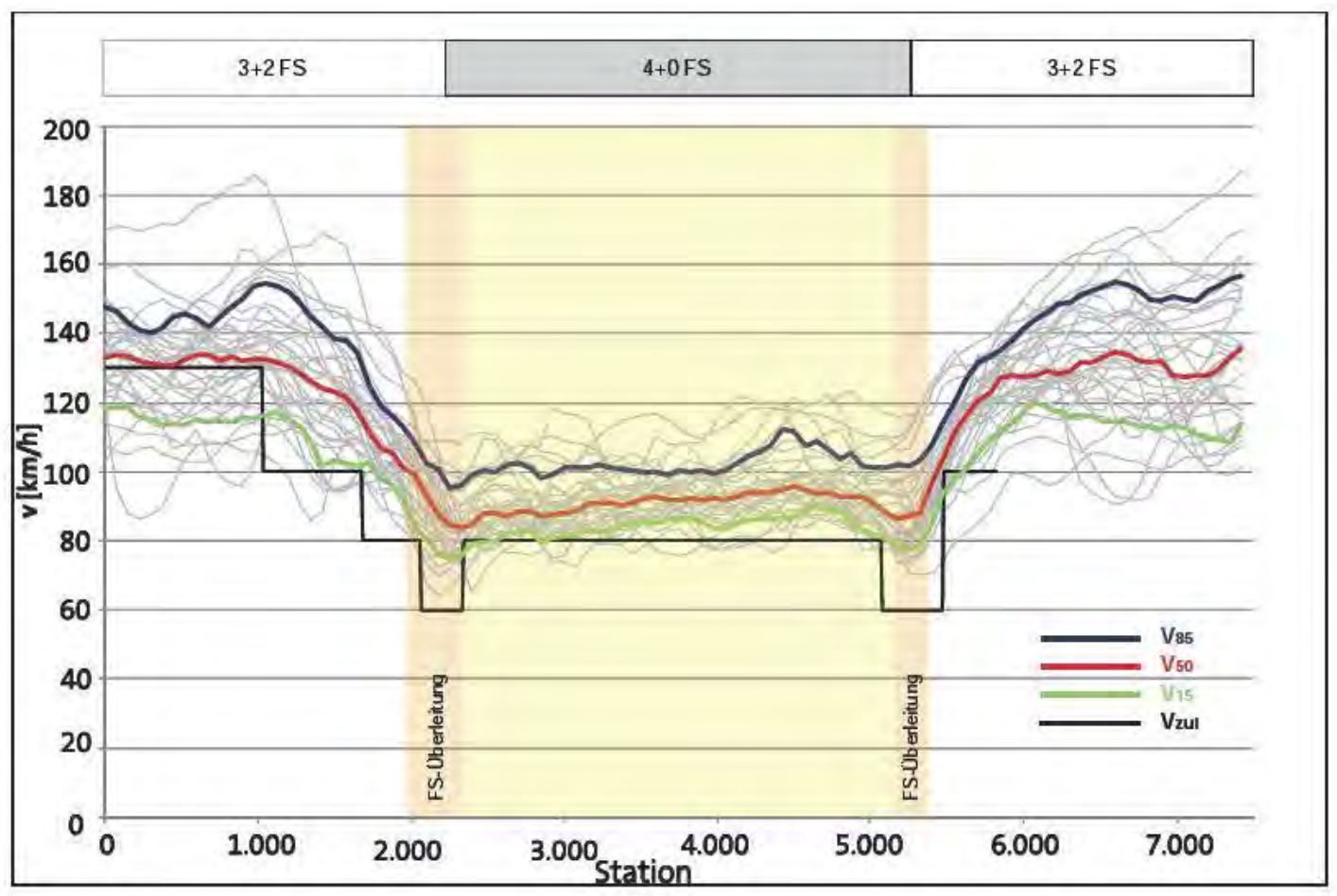

Abbildung C-1:

Geschwindigkeitsprofile in Fahrtrichtung 1, Zustand weiß, verkehrsschwach

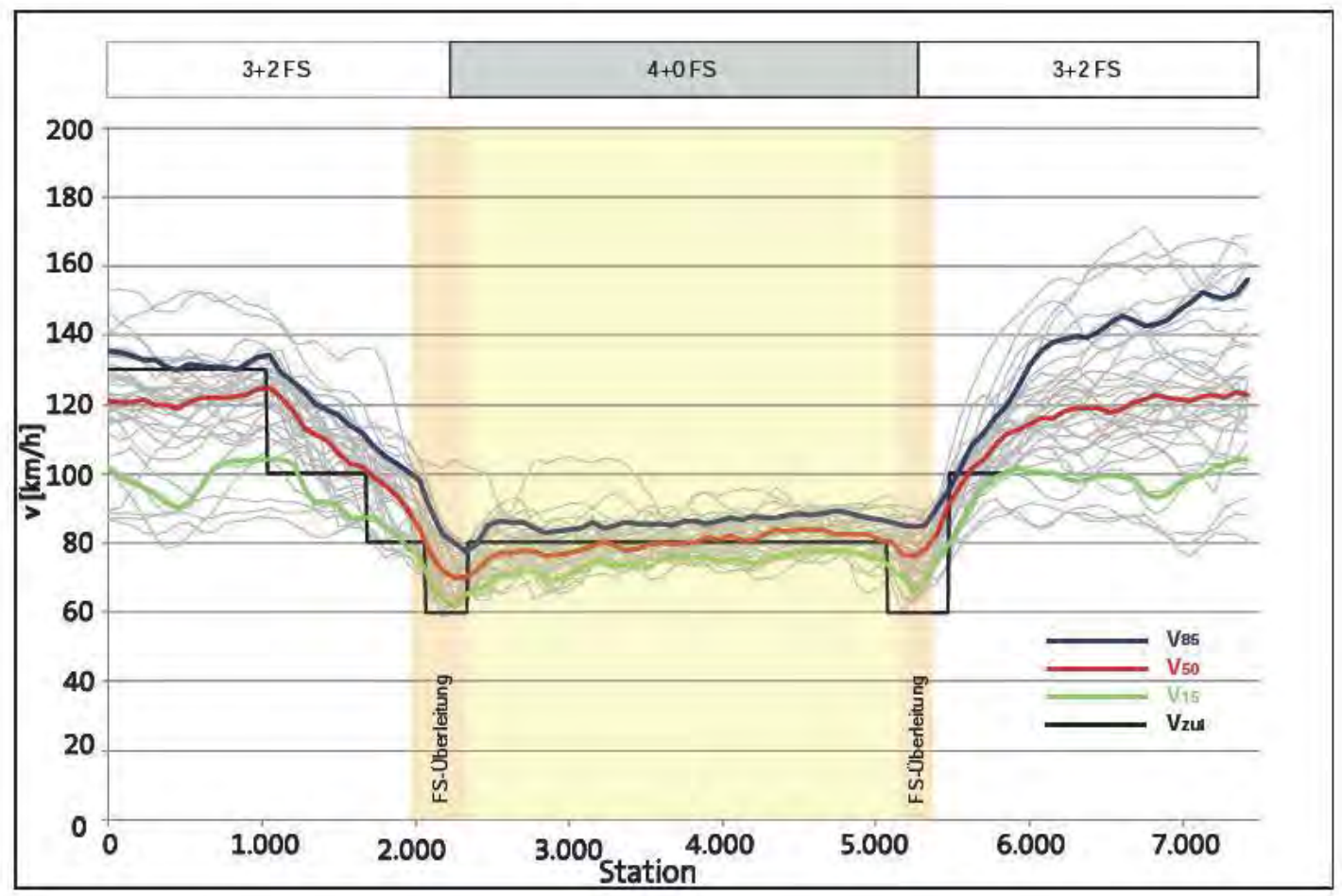

Abbildung C-2:

Geschwindigkeitsprofile in Fahrtrichtung 1, Zustand weiß, verkehrsstark 


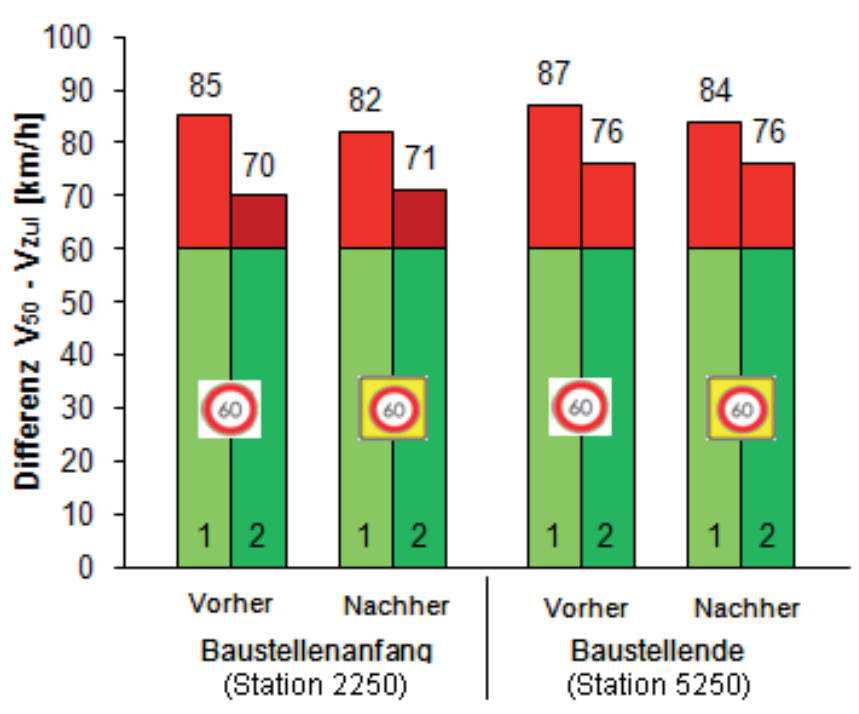

口zulässige Höchstgeschwindigkeit

1 verkehrsschwach

$\square$ durchschnittliche Überschreitung 2 verkehrsstark

Abbildung C-3:

Differenz der $v_{50} \mathbf{z u ~ v}_{\mathrm{zul}}$ in Fahrtrichtung 1

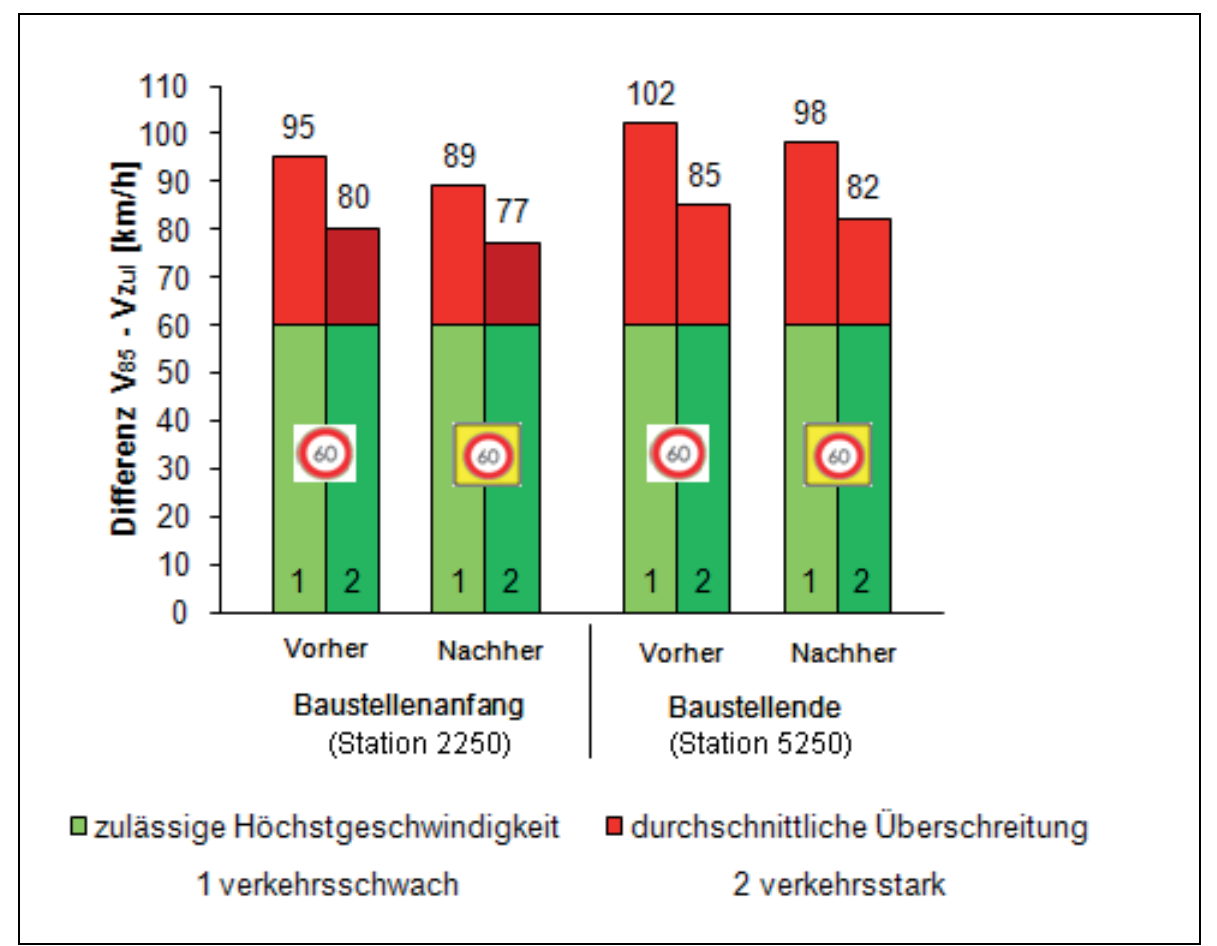

Abbildung C-4:

Differenz der $v_{85} z_{u} v_{z u l}$ in Fahrtrichtung 1 


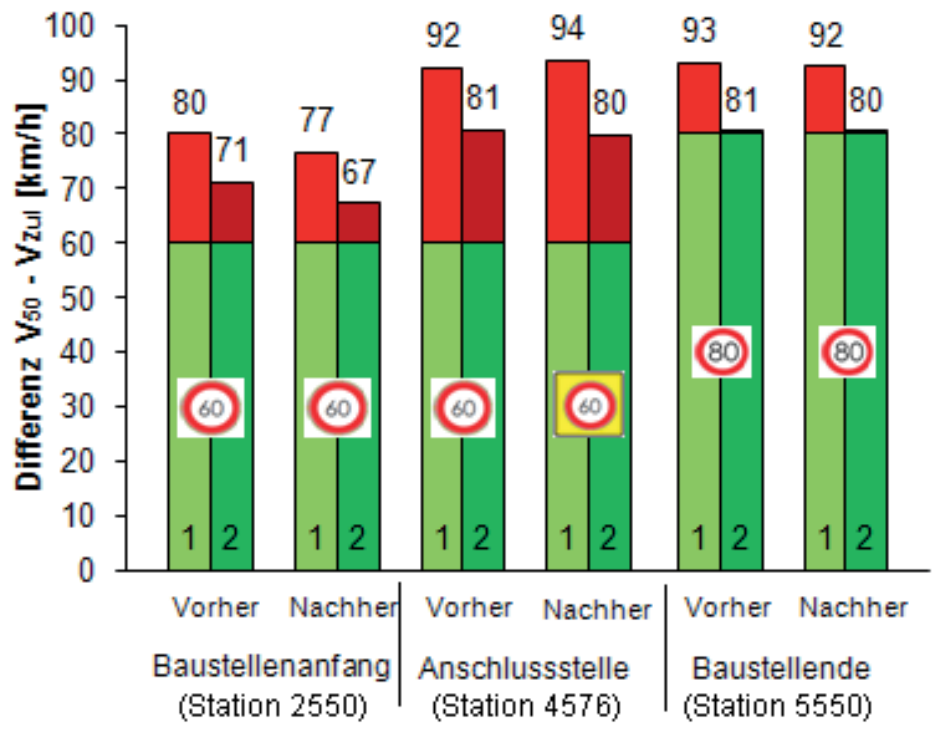

口zulässige Höchstgeschwindigkeit $\square$ durchschnittliche Überschreitung 1 verkehrsschwach 2 verkehrsstark

Abbildung C-5:

Differenz der $v_{50} z_{u} v_{z u l}$ in Fahrtrichtung 2

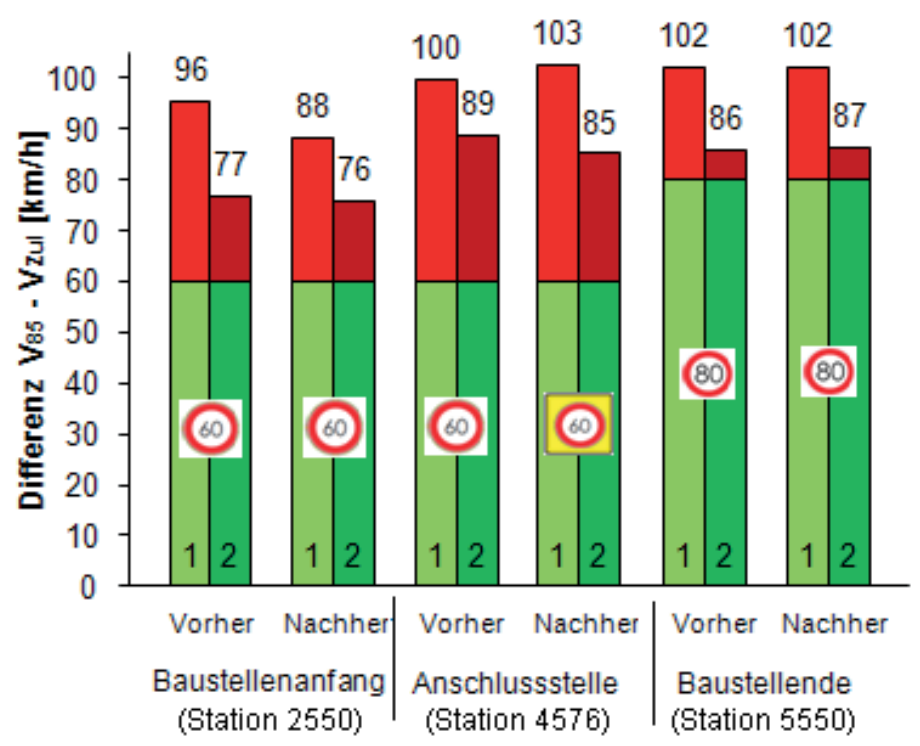

$\square$ zulässige Höchstgeschwindigkeit $\square$ durchschnittliche Überschreitung 1 verkehrsschwach 2 verkehrsstark 


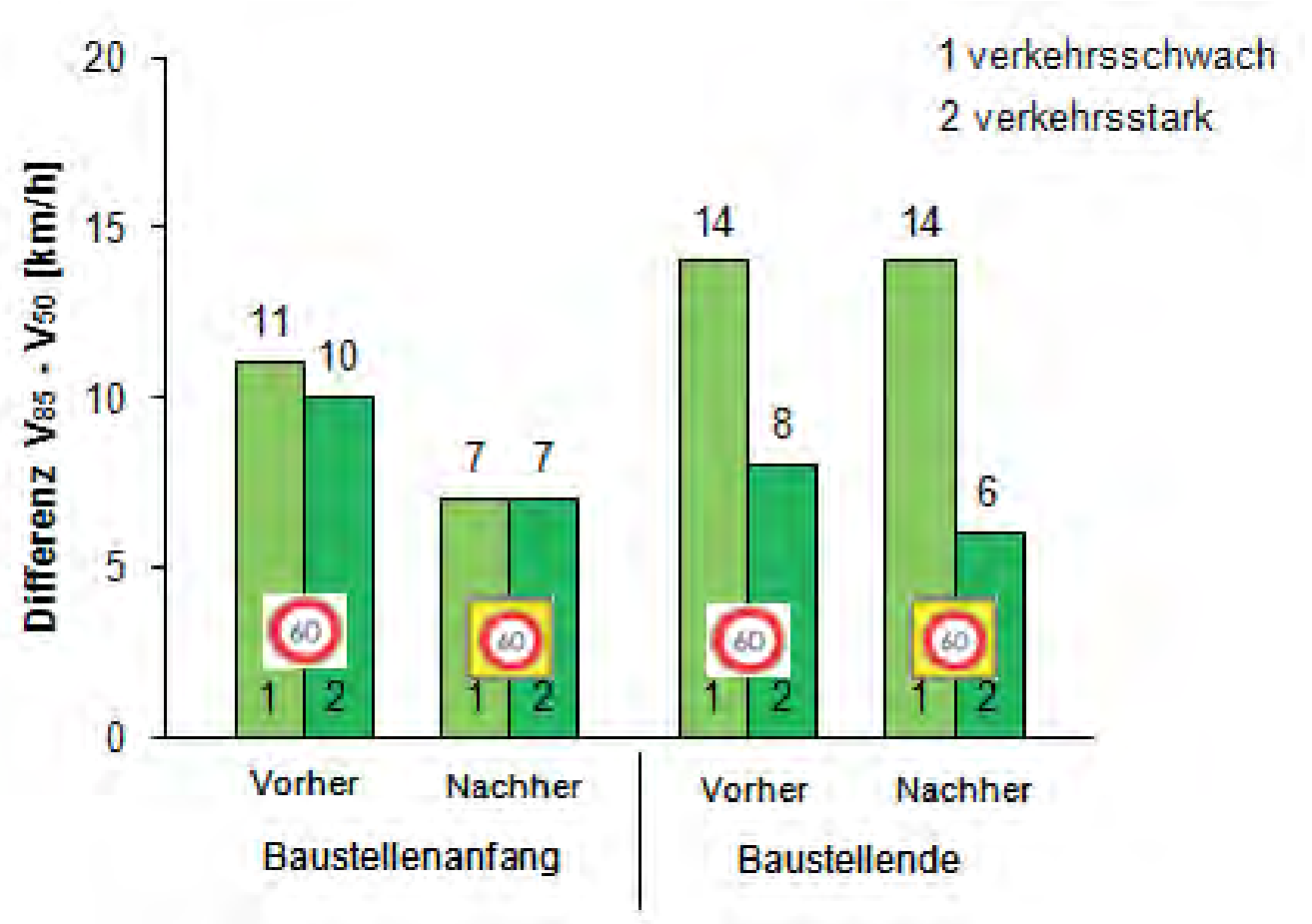

Abbildung C-7:

Homogenität der Geschwindigkeit in Fahrtrichtung 1

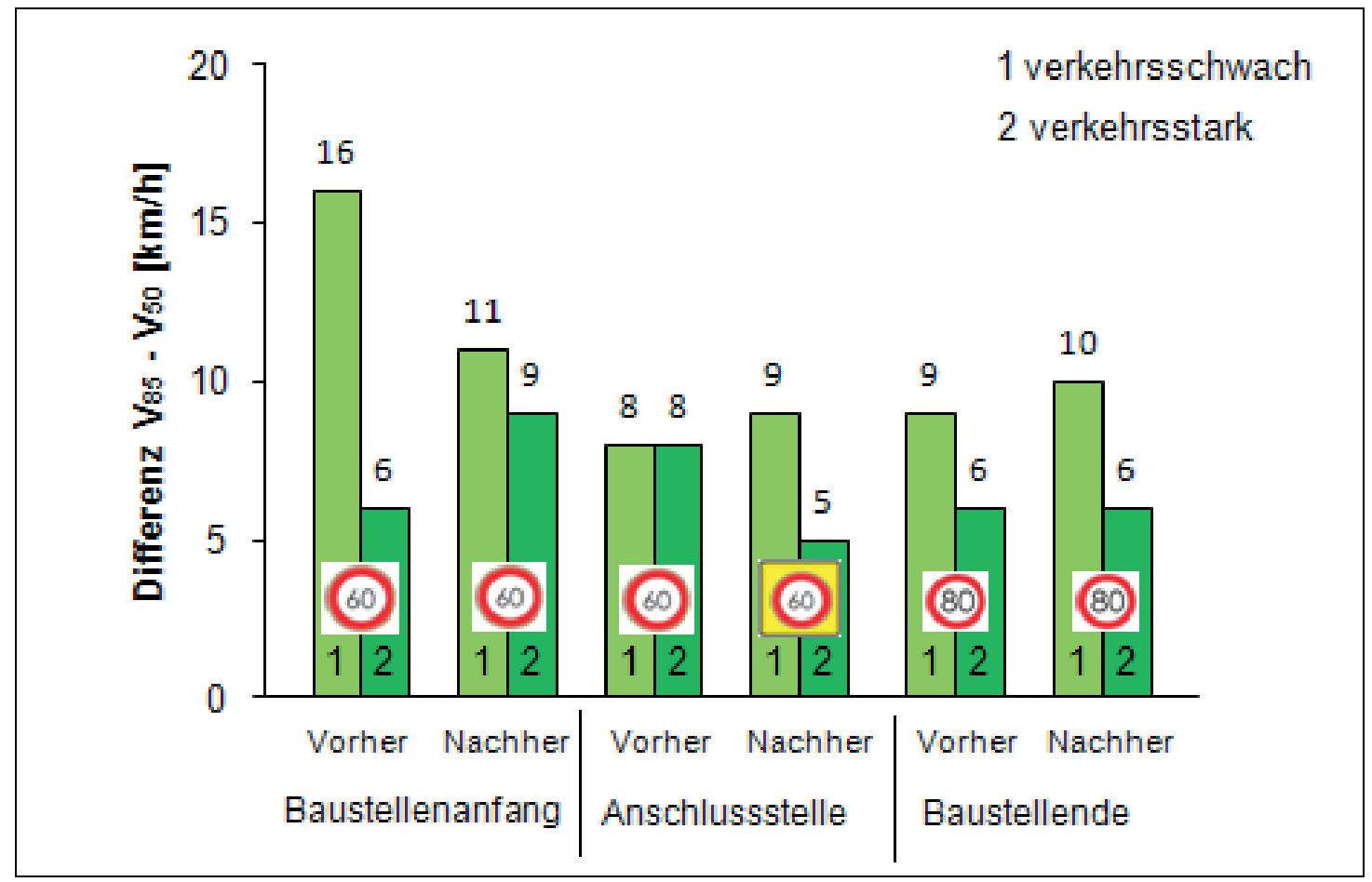

Abbildung C-8:

Homogenität der Geschwindigkeit in Fahrtrichtung 2 


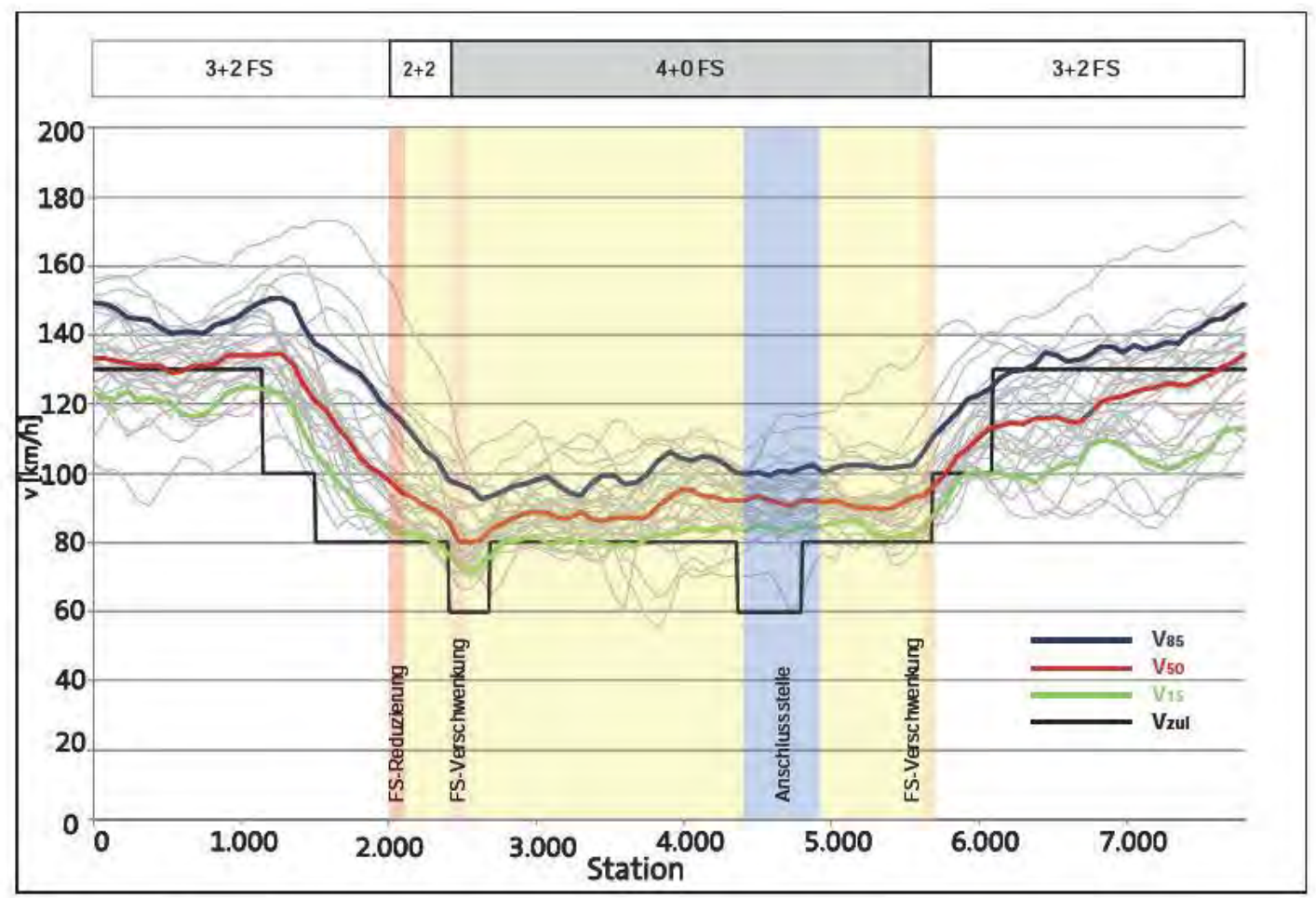

Abbildung C-9:

Geschwindigkeitsprofile in Fahrtrichtung 2, Zustand weiß, verkehrsschwach

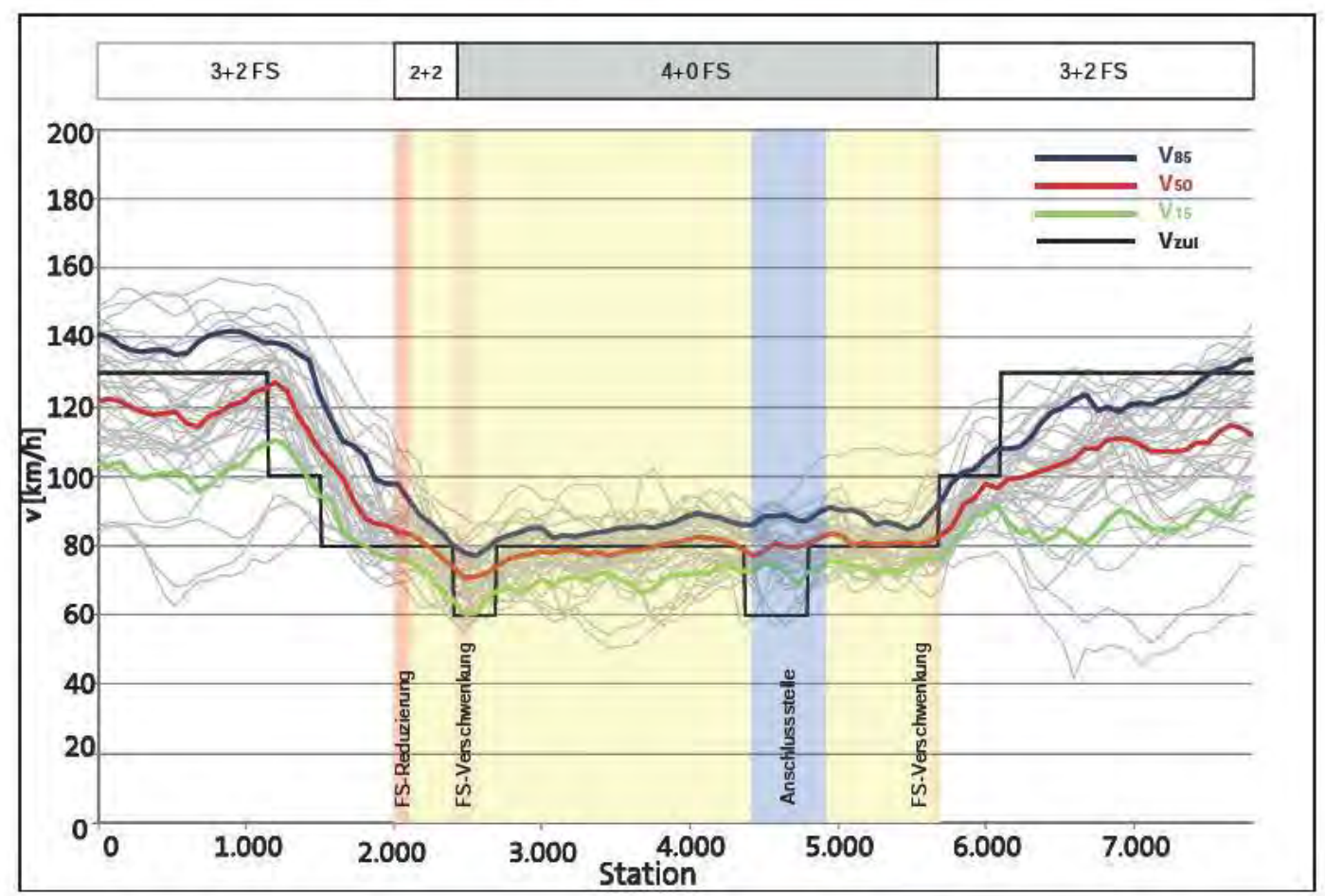

Abbildung C-10:

Geschwindigkeitsprofile in Fahrtrichtung 2, Zustand weiß, verkehrsstark 


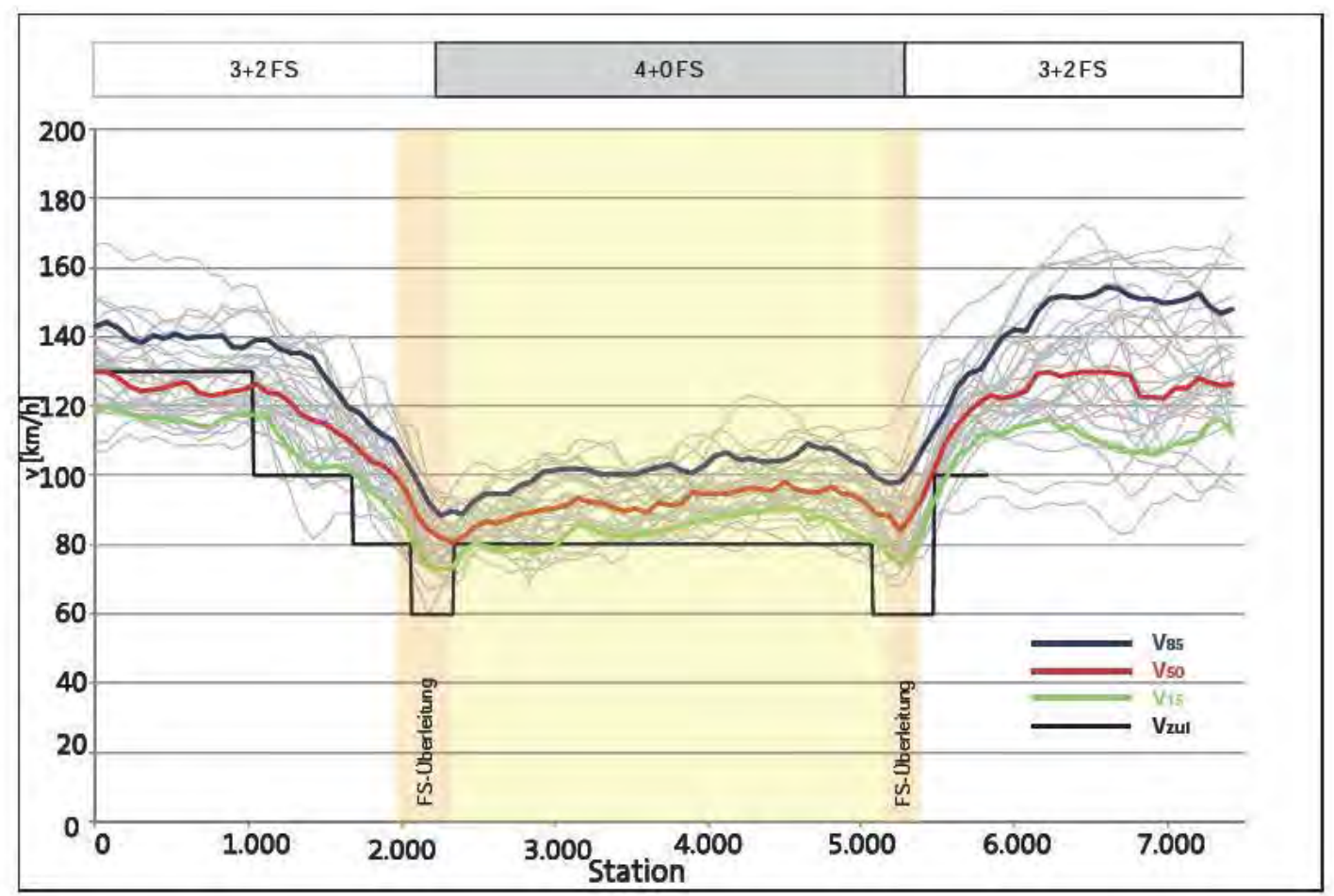

Abbildung C-11:

Geschwindigkeitsprofile in Fahrtrichtung 1, Zustand gelb, verkehrsschwach

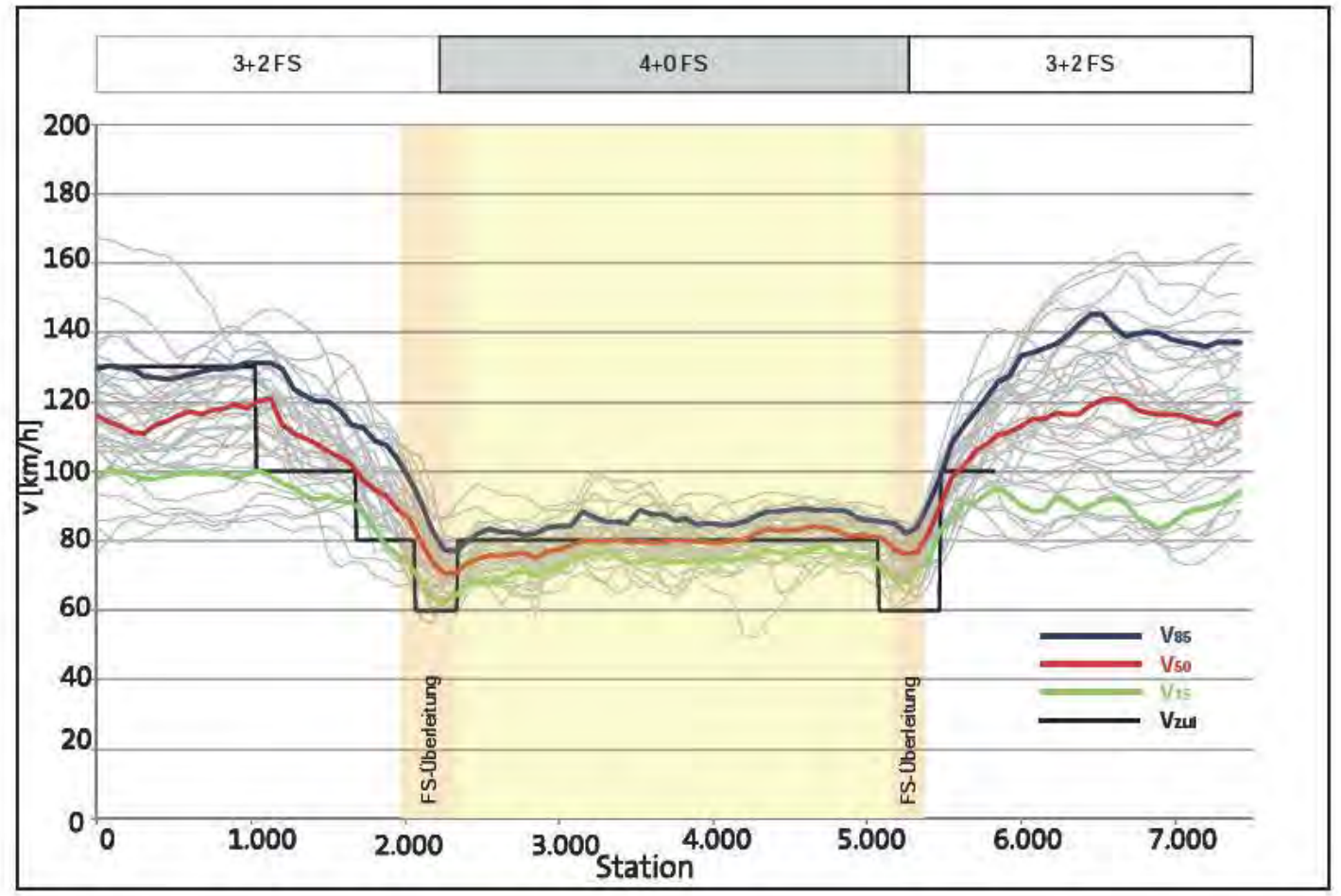

Abbildung C-12:

Geschwindigkeitsprofile in Fahrtrichtung 1, Zustand gelb, verkehrsstark 


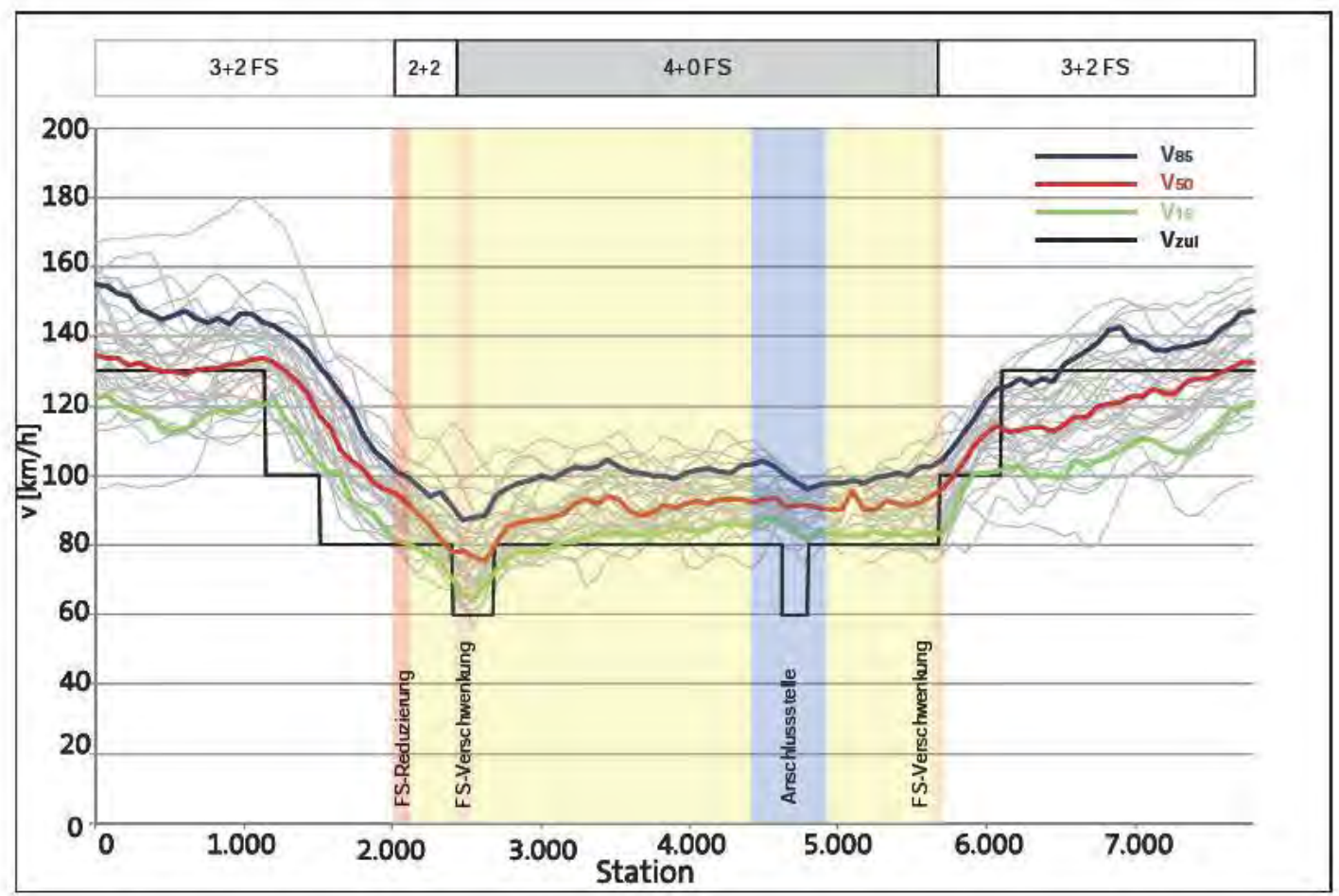

Abbildung $\mathrm{C}-13$

Geschwindigkeitsprofile in Fahrtrichtung 2, Zustand gelb, verkehrsschwach

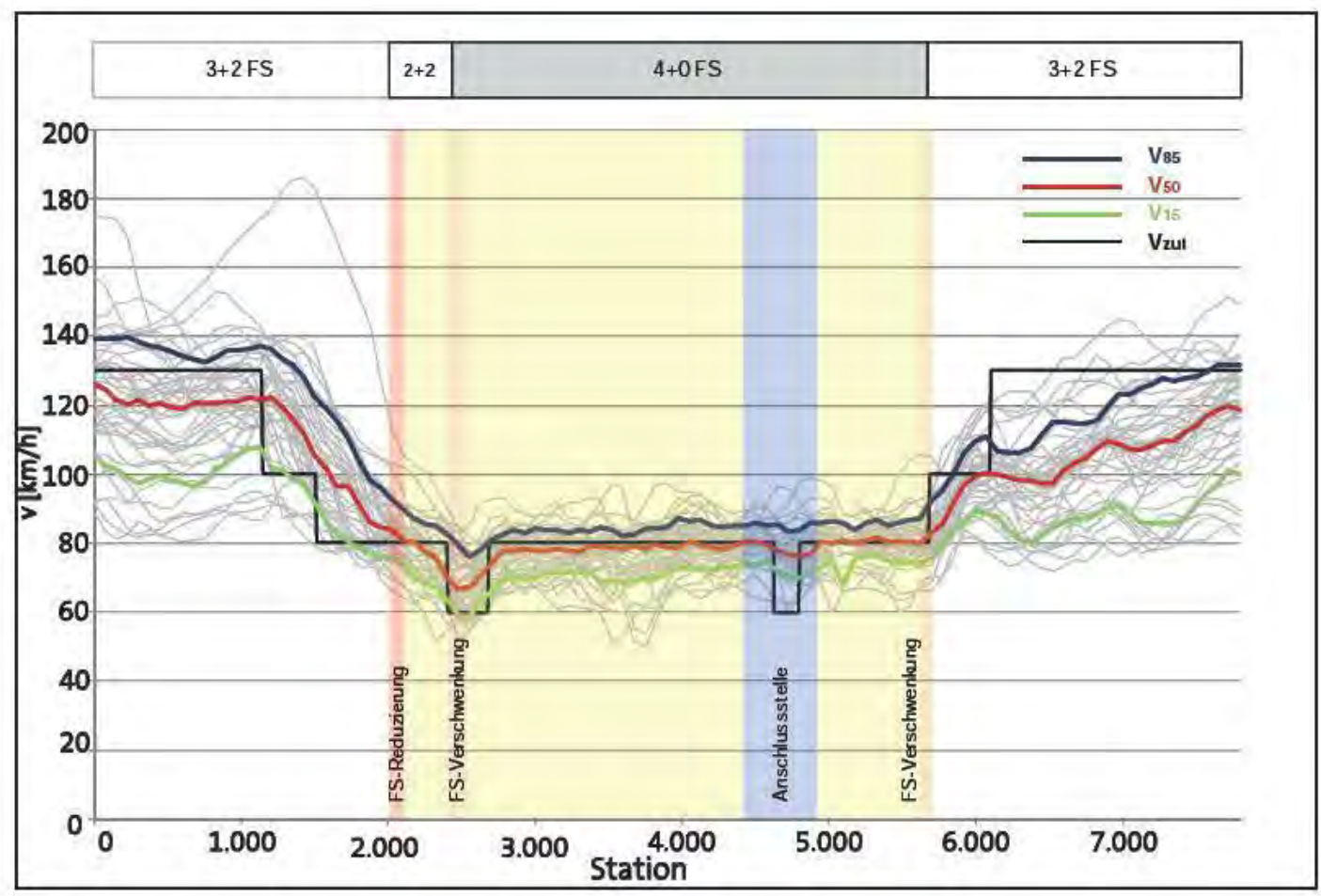

Abbildung C-14:

Geschwindigkeitsprofile in Fahrtrichtung 2, Zustand gelb, verkehrsstark 


\section{C.2 Annäherungsbereich}

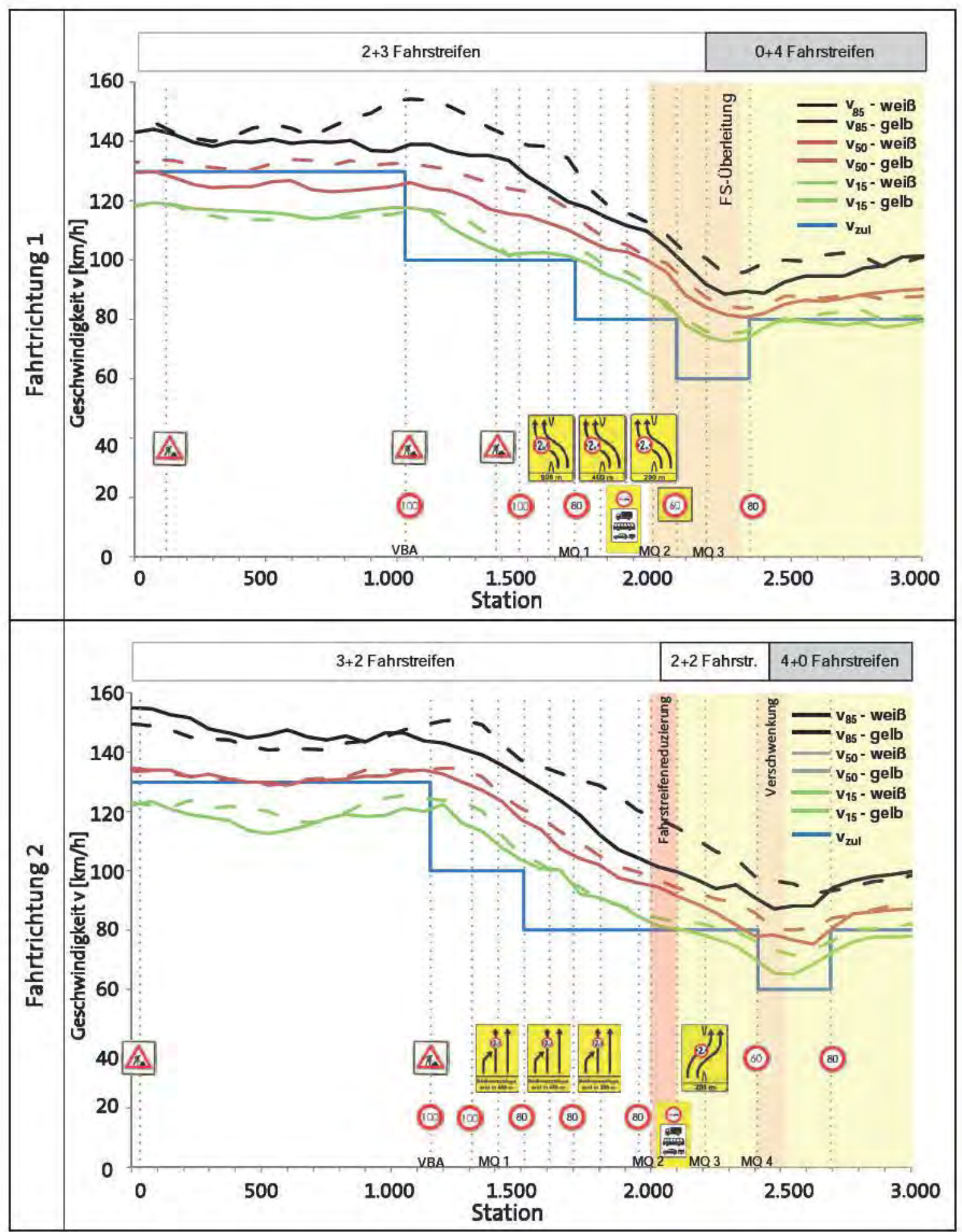

Abbildung C-15:

Verläufe der Geschwindigkeitskenngrößen $\mathbf{v}_{50}$ und $\mathbf{v}_{85}$ in den Annäherungsbereichen nach Beschilderungsart 


\section{C.3 Baustellenende}

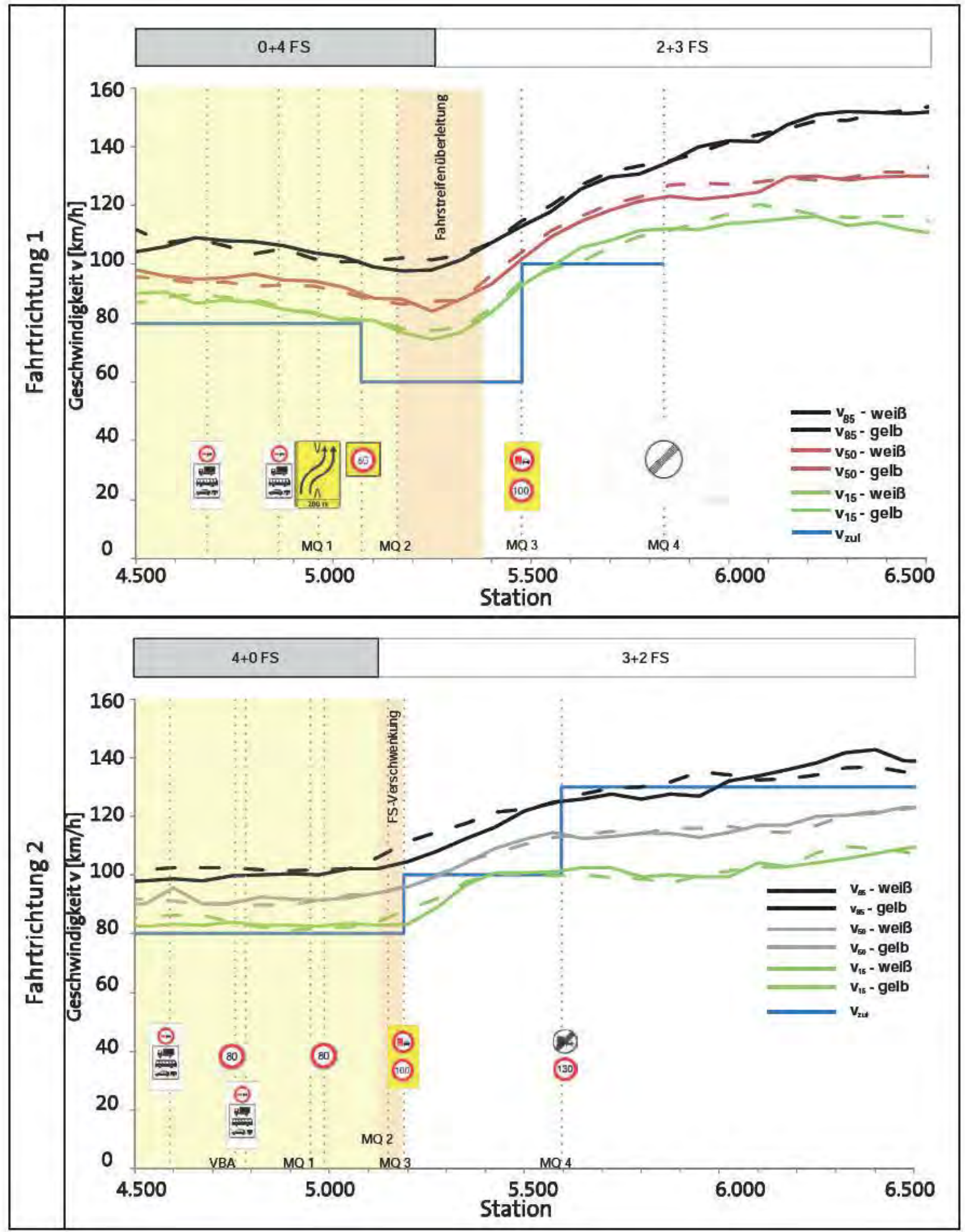

Abbildung C-16:

Verläufe der Geschwindigkeitskenngrößen $\mathbf{v}_{50}$ und $\mathbf{v}_{85}$ am Baustellenende nach Beschilderungsart 


\section{Freie Geschwindigkeiten im Baustellenbereich}

\section{D.1 Methodik}

\section{Definition der frei-fahrenden Fahrzeuge}

Fahrzeuge die auf demselben Fahrstreifen in gleicher Richtung fahren, können sich gegenseitig in ihrem Fahrverhalten beeinflussen. Insbesondere wenn die Längsabstände ein bestimmtes Maß unterschreiten wird das jeweilige Folgefahrzeug seine Geschwindigkeit dem Vorausfahrenden anpassen. In Schüller (2010) ${ }^{2}$ wurde das freie Fahren von Fahrzeugen über den zeitlichen Abstand zum jeweils vorausfahrenden Fahrzeug bestimmt. Unter Verwendung von Signifikanztests wurde der Zusammenhang von Abstand und Geschwindigkeit untersucht. Im Ergebnis zeigte sich, dass ab einer Lücke von mindestens 5 Sekunden nicht mehr von einer Beeinflussung des betrachteten Fahrzeugs durch den Vordermann zu rechnen ist. Da diese Untersuchung aber Innerortsstraßen zum Gegenstand hatte, wurde eine entsprechende Überprüfung auch für den Verkehrsablauf auf der A6 vorgenommen.

\section{Abstand zum vorausfahrenden Fahrzeug}

Zunächst wurde ein 30-Minuten-Intervall betrachtet (Tabelle D 2). Ein erster Vergleich zwischen den Fahrzeugen mit Abständen von mindestens 5 Sekunden und solchen mit mindestens 6 Sekunden ergab keinen signifikanten Unterschied zwischen den mittleren Geschwindigkeiten. Auch der Vergleich der mittleren Geschwindigkeiten von Fahrzeugen, welche 5 Sekunden mit solchen, die 10 Sekunden hinter einem vorausfahrenden Fahrzeug fuhren ergab keinen nachweisbaren Einfluss. Demnach müssen Fahrzeuge mit 5 Sekunden Längsabstand ebenso als frei fahrend angesehen werden, wie solche mit 10 Sekunden Abstand.

Bei zulässigen $80 \mathrm{~km} / \mathrm{h}$ im Baustellenbereich entspricht ein Zeitabstand von 5 Sekunden einer Raumlücke von ca. $110 \mathrm{~m}$.
Derzeit wurden an 28 Querschnitten jeweils 30 min Videomaterial ausgewertet ( $=14 \mathrm{~h}$ Videobeobachtungen. Unter Verwendung der 5 Sekunden-Grenze für die freien Geschwindigkeiten lassen sich die im Angebot angegebenen Kollektivgrößen (mindestens 80 frei fahrende Fahrzeuge) für jeden Fahrstreifen erreichen. Da zwischen Geschwindigkeiten bei unterschiedlichen Abständen (zwischen 5 und 10 Sekunden) kein signifikanter Unterschied feststellbar war, wird die Verwendung der 5 Sekunden-Grenze empfohlen. Insbesondere für den rechten Fahrstreifen ergeben sich bei hohen Verkehrsstärken aus der Wahl größerer Zeitlücken (10 s) sehr geringe Untersuchungskollektive.

Im Verlauf der Auswertungen wird der Einfluss des Längsabstands auf die gemessenen Geschwindigkeiten weiter überprüft. Sollten sich dabei neue Erkenntnisse ergeben, wird der gewählte zeitliche Abstand zur Definition der freien Geschwindigkeiten angepasst.

\section{$\underline{\text { Abstand zum Folgefahrzeug }}$}

Da auch folgende Fahrzeuge einen Einfluss auf die Geschwindigkeitswahl haben können wurde auch dieser Zusammenhang überprüft. Aus der vorangegangenen Überprüfung der Abstände zum Vordermann hat sich gezeigt, dass sich zwischen den Zeitabständen zwischen 5 und 10 Sekunden kein Einfluss auf die Wahl der gefahrenen Geschwindigkeiten nachweisen lässt. Um der weiteren Betrachtung ein möglichst großes Kollektiv zu Grunde legen zu können, wurde die Gruppe der Beobachtungsfälle mit mindestens 5 Sekunden Abstand zum Vorderfahrzeug betrachtet.

Den untersuchten Klassen, welche auf einer Mindestzeitlücke basieren ( $F z$, die mindestens 1 Sekunde vor dem Folgefahrzeug fuhren) wurde die Klasse der Fahrzeuge mit weniger als 1 Sekunde Abstand gegenübergestellt.

Ein Einfluss der (zeitlichen) Längsabstände zum Folgefahrzeug auf die gefahrene Geschwindigkeit kann nicht

2 Schüller, H.: Modelle zur Beschreibung des Geschwindigkeitsverhaltens auf Stadtstraßen und dessen Auswirkungen auf die Verkehrssicherheit auf Grundlage der Straßengestaltung. Dissertation. Fakultät Verkehrswissenschaften „Friedrich List“; Institut für Verkehrsplanung und Straßenverkehr; Professur für Straßenverkehrstechnik, Technische Universität Dresden; Dresden, 2010 
Tabelle D-1:

Einfluss Längsabstand auf die gefahrene Geschwindigkeit auf dem rechten Fahrstreifen im Baustellenbereich A6 Enkenbach-Alsenborn

\begin{tabular}{|c|c|c|c|c|c|c|c|}
\hline $\begin{array}{c}\text { Abstand } \\
\text { [s] }\end{array}$ & Anzahl Fz & $\begin{array}{c}\text { Mittlere } \\
\text { Geschwindigkeit } \\
\mathrm{v}_{\mathrm{m}} \\
{[\mathrm{km} / \mathrm{h}]}\end{array}$ & $\begin{array}{c}\text { Standard- } \\
\text { abweichung } \\
\mathrm{s}^{2}\end{array}$ & Freiheitsgrade & Prüf & jße & signifikant? \\
\hline 5 & 116 & 80,7 & 71,89 & \multirow{2}{*}{210} & \multirow{2}{*}{0,11} & \multirow{2}{*}{1,65} & \multirow{2}{*}{ nein } \\
\hline 6 & 96 & 80,6 & 66,29 & & & & \\
\hline 5 & 116 & 80,7 & 71,89 & \multirow{2}{*}{147} & \multirow{2}{*}{0,39} & \multirow{2}{*}{1,66} & \multirow{2}{*}{ nein } \\
\hline 10 & 33 & 81,4 & 52,14 & & & & \\
\hline
\end{tabular}

Tabelle D-2:

Einfluss Längsabstand auf die gefahrene Geschwindigkeit auf dem Überholfahrstreifen im Baustellenbereich A6 Enkenbach-Alsenborn

\begin{tabular}{|c|c|c|c|c|c|c|c|}
\hline Abstand & Anzahl Fz & Mittlere & Standard- & Freiheitsgrade & \multicolumn{2}{|c|}{ Prüfgröße } & Signifikant? \\
\hline$[\mathrm{s}]$ & & $\begin{array}{c}\mathrm{v}_{\mathrm{m}} \\
{[\mathrm{km} / \mathrm{h}]}\end{array}$ & $S^{2}$ & $\mathrm{~m}$ & $t^{*}$ & $\mathrm{t}$ & \\
\hline 5 & 73 & 94,4 & 143,40 & \multirow{2}{*}{135} & \multirow{2}{*}{0,22} & \multirow{2}{*}{1,65} & \multirow{2}{*}{ nein } \\
\hline 6 & 64 & 94,0 & 138,75 & & & & \\
\hline 5 & 73 & 94,4 & 143,40 & \multirow{2}{*}{119} & \multirow{2}{*}{0,98} & \multirow{2}{*}{1,66} & \multirow{2}{*}{ nein } \\
\hline 10 & 48 & 92,3 & 130,53 & & & & \\
\hline
\end{tabular}

Tabelle D-3:

Einfluss Längsabstand zum Folgefahrzeug auf die gefahrene Geschwindigkeit auf dem rechten Fahrstreifen im Baustellenbereich A6 Enkenbach-Alsenborn

\begin{tabular}{|c|c|c|c|c|c|c|c|c|c|}
\hline $\mathbf{t}_{\text {Vordermann }}$ & $t_{\text {folgefahrzeug }}$ & $\begin{array}{c}\text { Abstand } \\
{[\mathrm{m}]}\end{array}$ & Anzahl & $\mathbf{v}_{\mathbf{m}}$ & $\mathbf{s}^{2}$ & $\mathbf{m}$ & $t^{*}$ & $\mathbf{t}$ & Signifikant? \\
\hline \multirow[t]{4}{*}{5} & $<1 s$ & $<23$ & 16 & 82,7 & 66,73 & \multirow{2}{*}{113} & \multirow{2}{*}{1,01} & \multirow{2}{*}{1,66} & \multirow{2}{*}{ nein } \\
\hline & 1 & 22 & 99 & 80,4 & 72,67 & & & & \\
\hline & 1 & 22 & 99 & 80,4 & 72,67 & \multirow{2}{*}{133} & \multirow{2}{*}{0,21} & \multirow{2}{*}{1,66} & \multirow{2}{*}{ nein } \\
\hline & 5 & 112 & 36 & 80,7 & 77,73 & & & & \\
\hline
\end{tabular}

Tabelle D-4:

Einfluss Längsabstand zum Folgefahrzeug auf die gefahrene Geschwindigkeit auf dem linken Fahrstreifen im Baustellenbereich A6 Enkenbach-Alsenborn

\begin{tabular}{|c|c|c|c|c|c|c|c|c|c|}
\hline$t_{\text {Vordermann }}$ & $t_{\text {Folgefahrzeug }}$ & $\begin{array}{c}\text { Abstand } \\
{[\mathrm{m}]}\end{array}$ & Anzahl & $\mathbf{v}_{\mathrm{m}}$ & $\mathbf{s}^{2}$ & $\mathbf{m}$ & $t^{*}$ & $\mathbf{t}$ & Signifikant? \\
\hline \multirow[t]{4}{*}{5} & $<1 s$ & $<25$ & 9 & 89,9 & 215,96 & \multirow{2}{*}{69} & \multirow{2}{*}{$-1,22$} & \multirow{2}{*}{1,68} & \multirow{2}{*}{ nein } \\
\hline & 1 & 26 & 62 & 95,1 & 131,42 & & & & \\
\hline & 1 & 26 & 62 & 95,1 & 131,42 & \multirow{2}{*}{98} & \multirow{2}{*}{0,06} & \multirow{2}{*}{1,66} & \multirow{2}{*}{ nein } \\
\hline & 5 & 132 & 38 & 95,3 & 127,26 & & & & \\
\hline
\end{tabular}


nachgewiesen werden. Alle Unterschiede der Geschwindigkeitsmittelwerte sind nicht signifikant. Da aber die Werte der Klasse „„ 1 Sekunde“ deutlich von den weiteren Geschwindigkeiten abweichen, werden diese nicht in die Betrachtung der frei fahrenden Fahrzeuge einbezogen. Die Differenz der Geschwindigkeiten zwischen den fünf weiteren Kollektiven beträgt aber lediglich $1 \mathrm{~km} / \mathrm{h}$ (rechter Fahrstreifen) bzw. 0,6 km/h (linker Fahrstreifen).

Dass sich, im Gegensatz zu anderen Untersuchungen, hier kein Einfluss der rückwärtigen Abstands (Drängeln) auf die gefahrenen Geschwindigkeiten nachweisen lässt, ist sicherlich den vergleichsweise homogenen Geschwindigkeiten geschuldet.

\section{Fazit}

Aus der Betrachtung des Einflusses der Zeitlücken zwischen 5 und 10 Sekunden Länge zum vorausfahrenden Fahrzeug ergab sich kein nachweisbarer Einfluss auf die gemessenen mittleren Geschwindigkeiten. Im Gegensatz zu anderen Untersuchungen lässt sich auch für die Abstände zum Folgefahrzeug, welche etwa den Effekt des „Drängelns" beschreiben, kein Einfluss auf die Wahl der Geschwindigkeit nachweisen. Sicherlich besitzen diese Ergebnisse lediglich Gültigkeit für den hier untersuchten Baustellenbereich und sind im Kontext mit dem (gegenüber anderen Straßen) vergleichsweise homogenen Geschwindigkeitsverhalten zu interpretieren.

Auf Basis der Ergebnisse werden Fahrzeuge mit einem Abstand von mindestens 5 Sekunden zum Vordermann und über 1s zum Folgefahrzeug als frei-fahrend angesehen. Um aber die Unabhängigkeit der Geschwindigkeiten zu gewährleisten, erfolgen auch für die weiteren Querschnittsauswertungen entsprechende Signifikanztests.

\section{D.2 Ergebnisse}

\section{D.2.1 Allgemeines}

Den im Weiteren dargestellten Ergebnissen der Signifikanztests liegen die Geschwindigkeiten frei fahrender Fahrzeuge an den jeweiligen Messquerschnitten zu
Grunde. Diese wurden auf Basis der Längsabstände zu den vorausfahrenden und folgenden Fahrzeugen abgegrenzt. Dabei galten solche Fahrzeuge als frei fahrend, die zum Vordermann einen Abstand von mindestens $5 \mathrm{~s}$ und zum Folgefahrzeug mindestens $1 \mathrm{~s}$ einhielten. Die Abstände sind Ergebnis der in Anhang D durchgeführten Untersuchungen.

Gegenstand der Vergleiche, für die jeweils ein Signifikanzniveau von $\alpha=0,05$ angenommen wurde, sind die $v_{50}$.

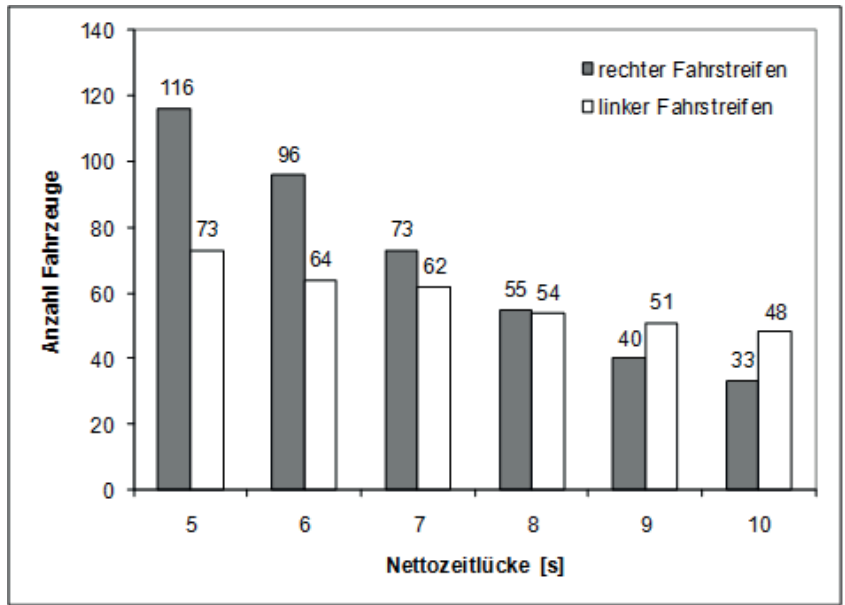

\section{Abbildung D-1:}

Anzahl frei-fahrender Fahrzeuge in Abhängigkeit der gewählten Größe der Nettozeitlücke - Auswertung eines 30 min-Intervalls

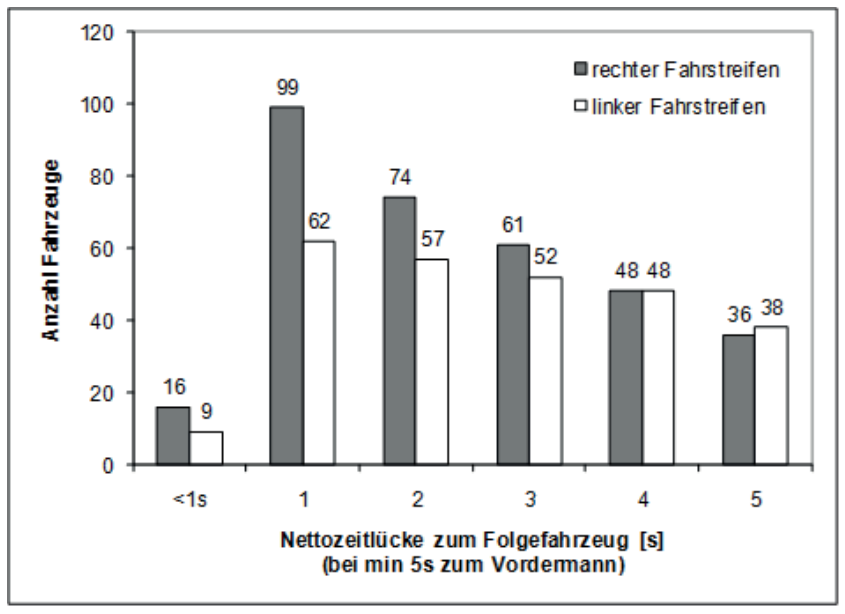

Abbildung D-2:

Anzahl frei-fahrender Fahrzeuge in Abhängigkeit der gewählten Größe der Nettozeitlücke zum Folgefahrzeug - Auswertung eines $\mathbf{3 0}$ min-Intervalls 
D.2.2 Annäherungsbereich

Fahrtrichtung 1

Tabelle D-5:

Geschwindigkeitskenngrößen im Annäherungsbereich Fahrtrichtung 1 Querschnitt 1

\begin{tabular}{|c|c|c|c|c|}
\hline & \multicolumn{2}{|c|}{ Fahrstreifen 1} & \multicolumn{2}{|c|}{ Fahrstreifen 2} \\
\hline & weiß & gelb & weiß & gelb \\
\hline Verkehrsstärke q & 406 & 384 & 184 & 186 \\
\hline$v_{m}$ & 107 & 101 & 115 & 113 \\
\hline $\mathrm{V}_{85}$ & 120 & 116 & 130 & 125 \\
\hline Anzahl freier Fz & 97 & 107 & 83 & 67 \\
\hline$s^{2}$ & 142,6 & 214,8 & 206,5 & 213,8 \\
\hline $\mathrm{m}$ & \multicolumn{2}{|c|}{202} & \multicolumn{2}{|c|}{148} \\
\hline$\hat{t}\left(\right.$ für $\left.v_{50}\right)$ & \multicolumn{2}{|c|}{3,13} & \multicolumn{2}{|c|}{0,88} \\
\hline$t($ für $\otimes=0,05)$ & \multicolumn{2}{|c|}{1,65} & \multicolumn{2}{|c|}{1,66} \\
\hline $\begin{array}{l}\text { Unterschied } \\
\text { signifikant? }\end{array}$ & \multicolumn{2}{|c|}{ ja } & \multicolumn{2}{|c|}{ nein } \\
\hline
\end{tabular}

Tabelle D-6:

Geschwindigkeitskenngrößen im Annäherungsbereich Fahrtrichtung 1 Querschnitt 2

\begin{tabular}{|c|c|c|c|c|}
\hline & \multicolumn{2}{|c|}{ Fahrstreifen 1} & \multicolumn{2}{|c|}{ Fahrstreifen 2} \\
\hline & weiß & gelb & weiß & gelb \\
\hline Verkehrsstärke q & 599 & 578 & 211 & 226 \\
\hline$v_{m}$ & 95 & 91 & 106 & 104 \\
\hline $\mathrm{V}_{85}$ & 108 & 101 & 116 & 116 \\
\hline Anzahl freier Fz & 139 & 151 & 84 & 58 \\
\hline$s^{2}$ & 135,9 & 180,1 & 165,6 & 160,5 \\
\hline $\mathrm{m}$ & \multicolumn{2}{|c|}{288} & \multicolumn{2}{|c|}{140} \\
\hline$\hat{t}\left(\right.$ für $v_{50}$ ) & \multicolumn{2}{|c|}{2,50} & \multicolumn{2}{|c|}{0,69} \\
\hline$t($ für $\otimes=0,05)$ & \multicolumn{2}{|c|}{1,65} & \multicolumn{2}{|c|}{1,66} \\
\hline $\begin{array}{l}\text { Unterschied } \\
\text { signifikant? }\end{array}$ & \multicolumn{2}{|c|}{ ja } & \multicolumn{2}{|c|}{ nein } \\
\hline
\end{tabular}


Tabelle D-7:

Geschwindigkeitskenngrößen im Annäherungsbereich Fahrtrichtung 1 Querschnitt 3

\begin{tabular}{|c|c|c|c|c|}
\hline \multicolumn{5}{|c|}{0 m vor der Fahrstreifenüberleitung } \\
\hline & \multicolumn{2}{|c|}{ Fahrstreifen 1} & \multicolumn{2}{|c|}{ Fahrstreifen 2} \\
\hline & weiß & gelb & weiß & gelb \\
\hline Verkehrsstärke q & 609 & 592 & 220 & 241 \\
\hline $\mathrm{v}_{\mathrm{m}}$ & 79 & 76 & 87 & 86 \\
\hline $\mathrm{V}_{85}$ & 90 & 85 & 101 & 101 \\
\hline Anzahl freier Fz & 135 & 135 & 66 & 58 \\
\hline$S^{2}$ & 102,4 & 108,0 & 101,6 & 162,7 \\
\hline $\mathrm{m}$ & \multicolumn{2}{|c|}{268} & \multicolumn{2}{|c|}{122} \\
\hline$\hat{t}\left(\right.$ für $\left.v_{50}\right)$ & \multicolumn{2}{|c|}{2,56} & \multicolumn{2}{|c|}{0,10} \\
\hline$t$ (für $\otimes=0,05$ ) & \multicolumn{2}{|c|}{1,65} & \multicolumn{2}{|c|}{1,66} \\
\hline $\begin{array}{l}\text { Unterschied } \\
\text { signifikant? }\end{array}$ & \multicolumn{2}{|c|}{ ja } & \multicolumn{2}{|c|}{ nein } \\
\hline
\end{tabular}




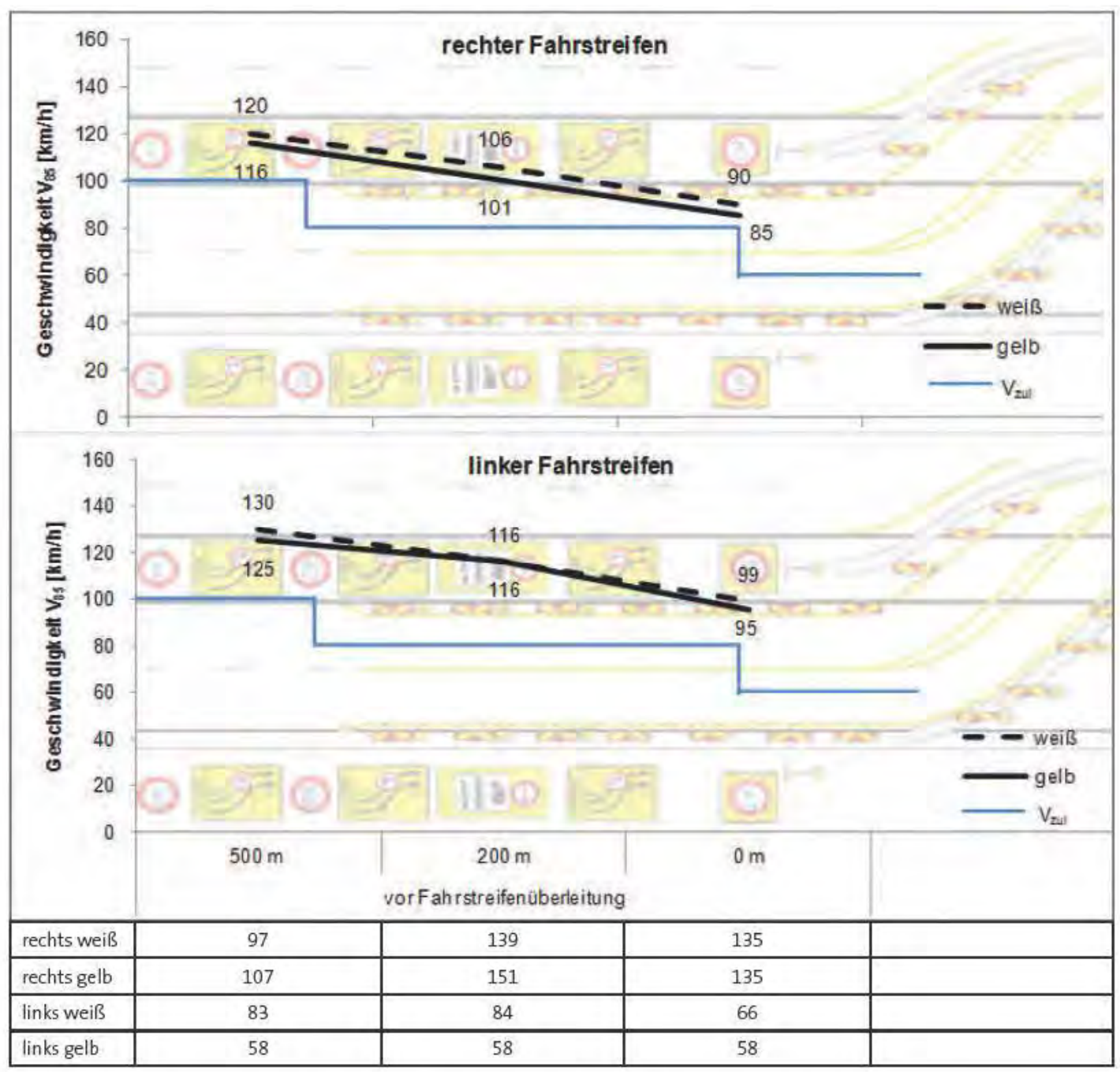

Abbildung D-3:

Geschwindigkeiten $\mathrm{V}_{85}$ im Annäherungsbereich der Fahrtrichtung 1 nach Fahrstreifen mit dem zu Grunde liegenden Untersuchungskollektiv (Tabelle)

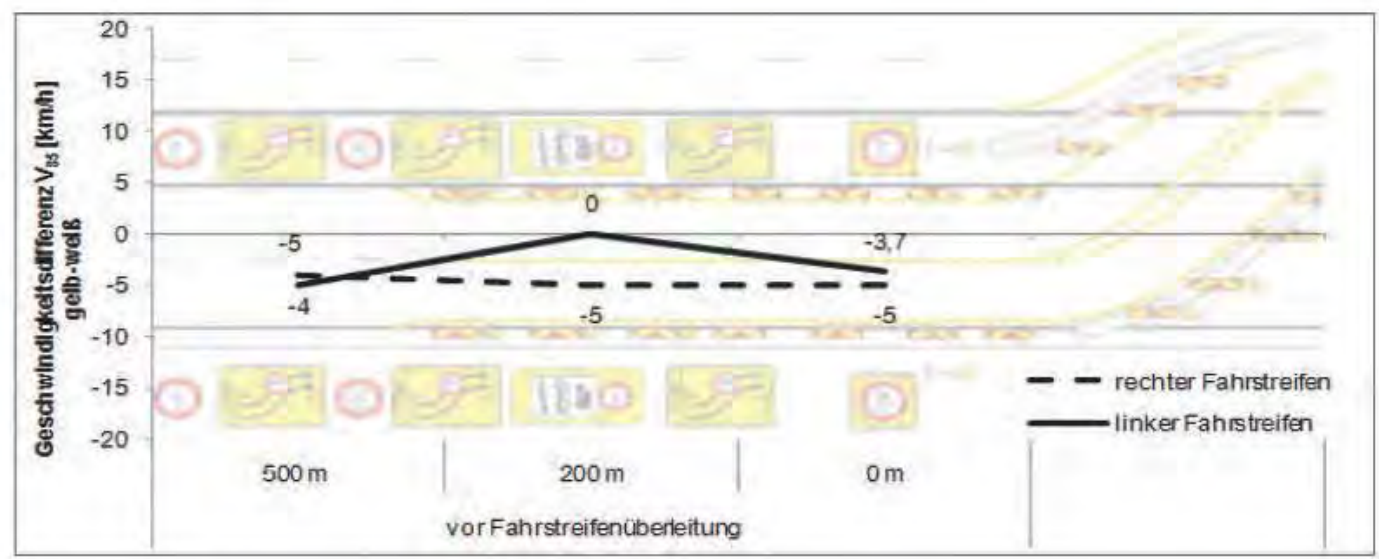

Abbildung D-4:

Differenz der Geschwindigkeiten $\mathrm{V}_{\mathbf{8 5}}$ der Untersuchungsphasen im Annäherungsbereich der Fahrtrichtung 1 nach Fahrstreifen 


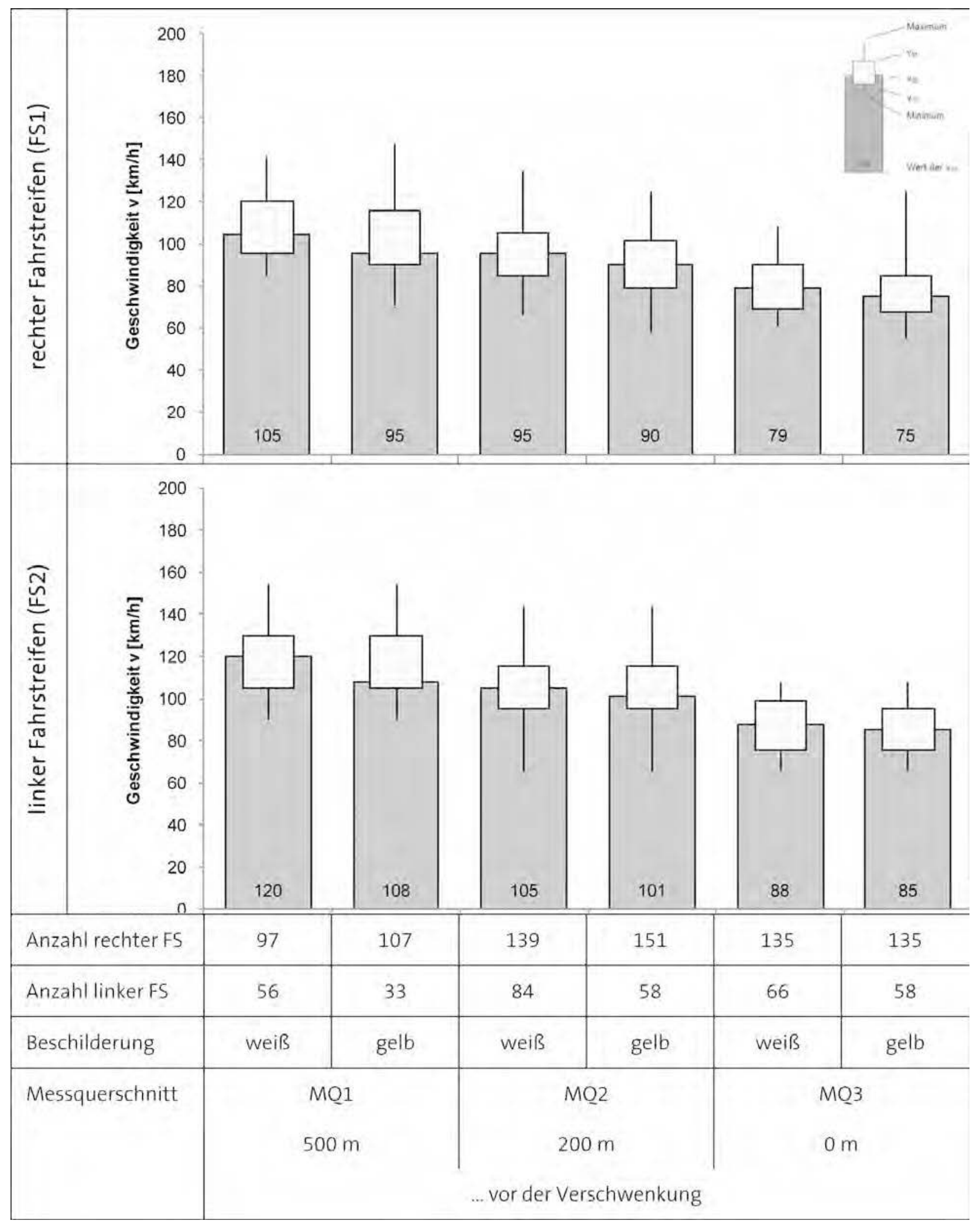

Abbildung D-5:

Kenngrößen der Geschwindigkeitsverteilung an den Messquerschnitten im Annäherungsbereich der Fahrtrichtung 1 


\section{Fahrtrichtung 2}

Tabelle D-8:

Geschwindigkeitskenngrößen im Annäherungsbereich Fahrtrichtung 2 Querschnitt 1

\section{$600 \mathrm{~m}$ vor der Reduktion der Fahrstreifen}

\begin{tabular}{|c|c|c|c|c|}
\hline & \multicolumn{2}{|c|}{ Fahrstreifen 1} & \multicolumn{2}{|c|}{ Fahrstreifen 2} \\
\hline & weiß & gelb & weiß & gelb \\
\hline Verkehrsstärke q & 309 & 303 & 190 & 215 \\
\hline$v_{m}$ & 99 & 102 & 123 & 130 \\
\hline $\mathrm{V}_{85}$ & 117 & 116 & 142 & 147 \\
\hline Anzahl freier Fz & 120 & 124 & 93 & 68 \\
\hline$S^{2}$ & 231,5 & 201,8 & 308,8 & 365,0 \\
\hline $\mathrm{m}$ & \multicolumn{2}{|c|}{242} & \multicolumn{2}{|c|}{159} \\
\hline$\hat{t}\left(\right.$ für $\left.v_{50}\right)$ & \multicolumn{2}{|c|}{1,70} & \multicolumn{2}{|c|}{2,30} \\
\hline$t($ für $\otimes=0,05)$ & \multicolumn{2}{|c|}{1,65} & \multicolumn{2}{|c|}{1,66} \\
\hline $\begin{array}{l}\text { Unterschied } \\
\text { signifikant? }\end{array}$ & \multicolumn{2}{|c|}{ ja } & \multicolumn{2}{|c|}{ ja } \\
\hline
\end{tabular}

Tabelle D-9:

Geschwindigkeitskenngrößen im Annäherungsbereich Fahrtrichtung 2 Querschnitt 2

\section{0 m vor der Reduktion der Fahrstreifen}

\begin{tabular}{|c|c|c|c|c|}
\hline & \multicolumn{2}{|c|}{ Fahrstreifen 1} & \multicolumn{2}{|c|}{ Fahrstreifen 2} \\
\hline & weiß & gelb & weiß & gelb \\
\hline Verkehrsstärke q & 359 & 400 & 182 & 172 \\
\hline$v_{m}$ & 83 & 83 & 94 & 101 \\
\hline $\mathrm{V}_{85}$ & 92 & 90 & 101 & 116 \\
\hline Anzahl freier Fz & 99 & 112 & 82 & 83 \\
\hline$s^{2}$ & 82,2 & 60,4 & 162,8 & 243,1 \\
\hline$m$ & \multicolumn{2}{|c|}{209} & \multicolumn{2}{|c|}{163} \\
\hline$\hat{t}\left(\right.$ für $\left.\mathrm{v}_{50}\right)$ & \multicolumn{2}{|c|}{0,35} & \multicolumn{2}{|c|}{3,11} \\
\hline $\mathrm{t}($ für $\otimes=0,05)$ & \multicolumn{2}{|c|}{1,65} & \multicolumn{2}{|c|}{1,66} \\
\hline $\begin{array}{l}\text { Unterschied } \\
\text { signifikant? }\end{array}$ & \multicolumn{2}{|c|}{ nein } & \multicolumn{2}{|c|}{ ja } \\
\hline
\end{tabular}


Tabelle D-10:

Geschwindigkeitskenngrößen im Annäherungsbereich Fahrtrichtung 2 Querschnitt 3

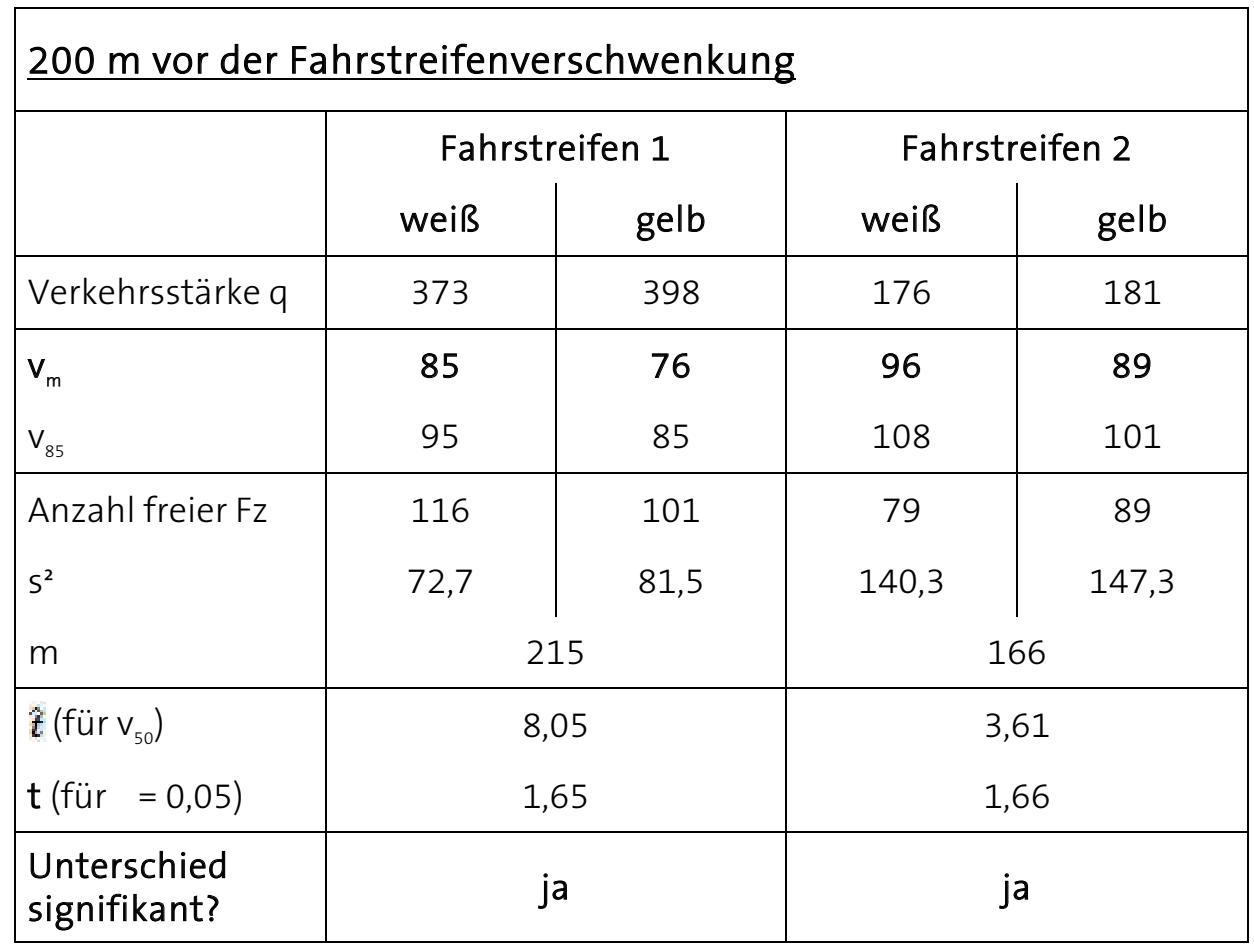

Tabelle D-11:

Geschwindigkeitskenngrößen im Annäherungsbereich Fahrtrichtung 2 Querschnitt 4

\begin{tabular}{|c|c|c|c|c|}
\hline & \multicolumn{2}{|c|}{ Fahrstreifen 1} & \multicolumn{2}{|c|}{ Fahrstreifen 2} \\
\hline & weiß & gelb & weiß & gelb \\
\hline Verkehrsstärke q & 451 & 382 & 244 & 177 \\
\hline$v_{m}$ & 80 & 71 & 90 & 80 \\
\hline $\mathrm{V}_{85}$ & 90 & 79 & 101 & 90 \\
\hline Anzahl freier Fz & 89 & 104 & 72 & 82 \\
\hline $\mathrm{s}^{2}$ & 81,9 & 106,0 & 99,8 & 193,9 \\
\hline $\mathrm{m}$ & \multicolumn{2}{|c|}{191} & \multicolumn{2}{|c|}{152} \\
\hline$\hat{t}$ (für $\mathrm{v}_{50}$ ) & \multicolumn{2}{|c|}{6,68} & \multicolumn{2}{|c|}{5,01} \\
\hline 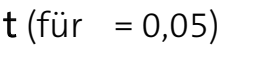 & \multicolumn{2}{|c|}{1,66} & \multicolumn{2}{|c|}{1,66} \\
\hline $\begin{array}{l}\text { Unterschied } \\
\text { signifikant? }\end{array}$ & \multicolumn{2}{|c|}{ ja } & \multicolumn{2}{|c|}{ ja } \\
\hline
\end{tabular}




\begin{tabular}{|cr|r|r|r|r|}
\hline & 150 & \multicolumn{5}{|c|}{ rechter Fahrstreifen } \\
\hline & 125 & & & & \\
\hline \\
\hline
\end{tabular}

Abbildung D-6:

Geschwindigkeiten $\mathbf{v}_{85}$ im Annäherungsbereich der Fahrtrichtung 2 nach Fahrstreifen mit dem zu Grunde liegenden Untersuchungskollektiv (Tabelle)

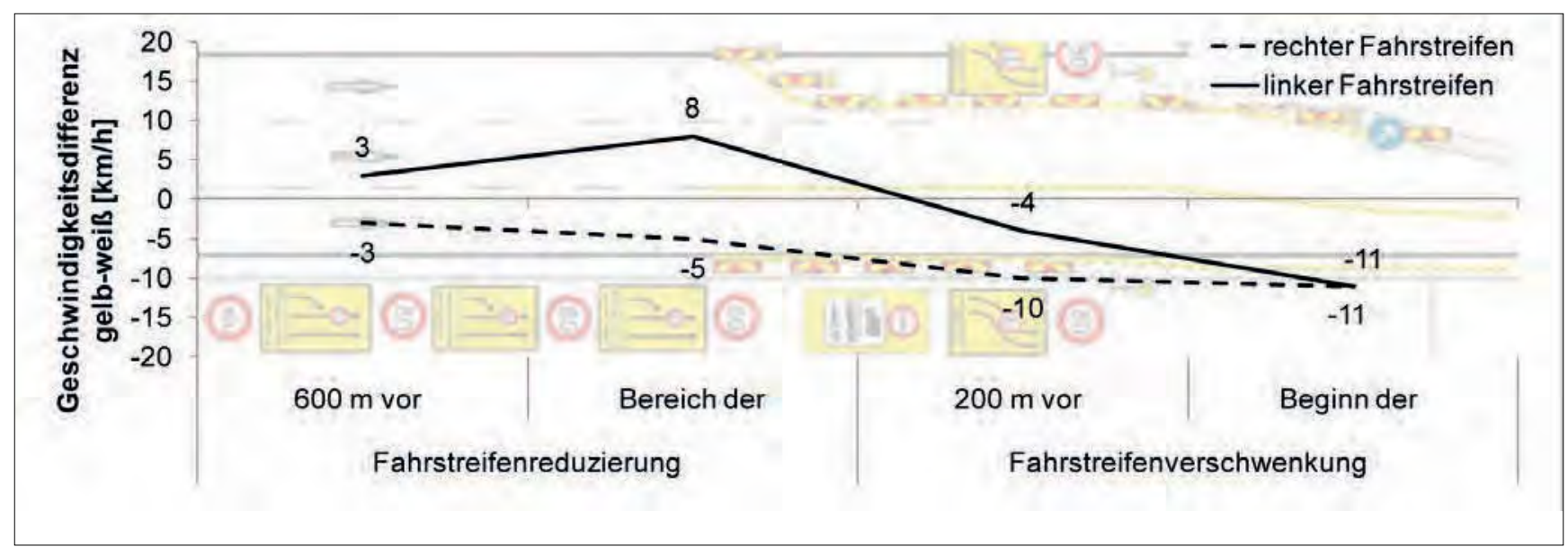

Abbildung D-7:

Differenz der Geschwindigkeiten $\mathbf{v}_{85}$ der Untersuchungsphasen im Annäherungsbereich der Fahrtrichtung 2 nach Fahrstreifen 


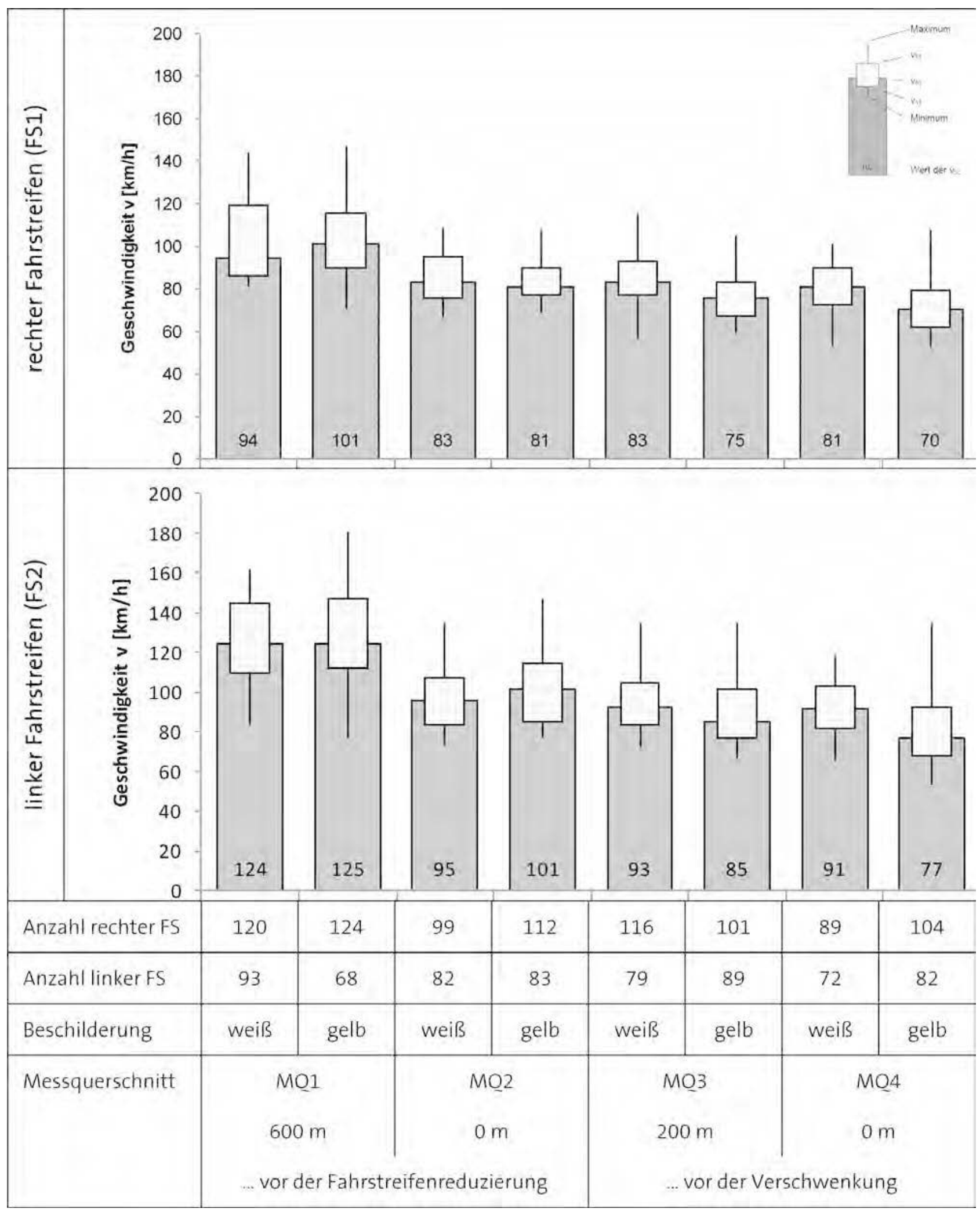

Abbildung D-8:

Kenngrößen der Geschwindigkeitsverteilung an den Messquerschnitten im Annäherungsbereich der Fahrtrichtung 2 
D.2.3 Baustellenende

Fahrtrichtung 1

Tabelle D-12:

Geschwindigkeitskenngrößen am Baustellende Fahrtrichtung 1 Querschnitt 1

\begin{tabular}{|c|c|c|c|c|}
\hline & \multicolumn{2}{|c|}{ Fahrstreifen 1} & \multicolumn{2}{|c|}{ Fahrstreifen 2} \\
\hline & weiß & gelb & weiß & gelb \\
\hline Verkehrsstärke q & 382 & 401 & 145 & 157 \\
\hline $\mathrm{v}_{\mathrm{m}}$ & 79 & 78 & 92 & 90 \\
\hline $\mathrm{V}_{85}$ & 85 & 89 & 108 & 95 \\
\hline Anzahl freier Fz & 81 & 83 & 81 & 81 \\
\hline$S^{2}$ & 71,5 & 115,6 & 156,6 & 112,3 \\
\hline $\mathrm{m}$ & \multicolumn{2}{|c|}{162} & \multicolumn{2}{|c|}{160} \\
\hline$\hat{t}\left(\right.$ für $\left.v_{50}\right)$ & \multicolumn{2}{|c|}{1,06} & \multicolumn{2}{|c|}{1,04} \\
\hline 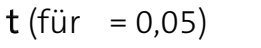 & \multicolumn{2}{|c|}{1,66} & \multicolumn{2}{|c|}{1,66} \\
\hline $\begin{array}{l}\text { Unterschied } \\
\text { signifikant? }\end{array}$ & \multicolumn{2}{|c|}{ nein } & \multicolumn{2}{|c|}{ nein } \\
\hline
\end{tabular}

Tabelle D-13:

Geschwindigkeitskenngrößen am Baustellende Fahrtrichtung 1 Querschnitt 2

\begin{tabular}{|c|c|c|c|c|}
\hline & \multicolumn{2}{|c|}{ Fahrstreifen 1} & \multicolumn{2}{|c|}{ Fahrstreifen 2} \\
\hline & weiß & gelb & weiß & gelb \\
\hline Verkehrsstärke q & 450 & 396 & 181 & 152 \\
\hline $\mathrm{v}_{\mathrm{m}}$ & 77 & 75 & 88 & 92 \\
\hline $\mathrm{V}_{85}$ & 86 & 90 & 101 & 101 \\
\hline Anzahl freier Fz & 80 & 84 & 56 & 59 \\
\hline$S^{2}$ & 164,9 & 173,8 & 180,7 & 132,8 \\
\hline $\mathrm{m}$ & \multicolumn{2}{|c|}{162} & \multicolumn{2}{|c|}{113} \\
\hline$\hat{t}\left(\right.$ für $\left.v_{50}\right)$ & \multicolumn{2}{|c|}{0,74} & \multicolumn{2}{|c|}{1,42} \\
\hline $\mathrm{t}($ für $\mathbb{B}=0,05)$ & \multicolumn{2}{|c|}{1,66} & \multicolumn{2}{|c|}{1,66} \\
\hline $\begin{array}{l}\text { Unterschied } \\
\text { signifikant? }\end{array}$ & \multicolumn{2}{|c|}{ nein } & \multicolumn{2}{|c|}{ nein } \\
\hline
\end{tabular}


Tabelle D-14:

Geschwindigkeitskenngrößen am Baustellende Fahrtrichtung 1 Querschnitt 3

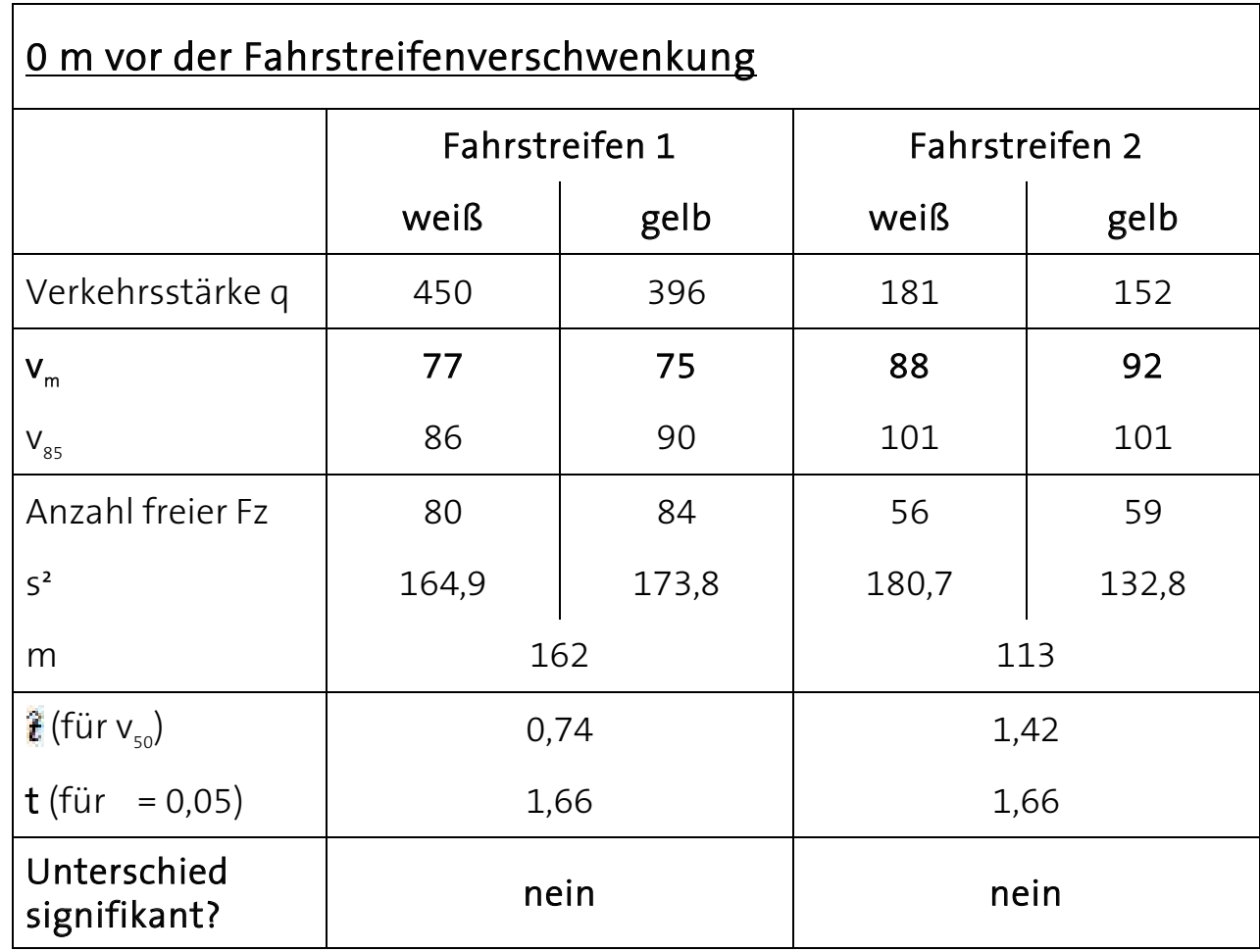

Tabelle D-15:

Geschwindigkeitskenngrößen am Baustellende Fahrtrichtung 1 Querschnitt 4

Zeichen 282 (Ende sämtlicher Streckenverbote)

\begin{tabular}{|c|c|c|c|c|}
\hline & \multicolumn{2}{|c|}{ Fahrstreifen 1} & \multicolumn{2}{|c|}{ Fahrstreifen 2} \\
\hline & weiß & gelb & weiß & gelb \\
\hline Verkehrsstärke q & 299 & 310 & 175 & 195 \\
\hline $\mathrm{v}_{\mathrm{m}}$ & 108 & 102 & 134 & 133 \\
\hline $\mathrm{V}_{85}$ & 135 & 125 & 147 & 147 \\
\hline Anzahl freier Fz & 99 & 102 & 78 & 78 \\
\hline$s^{2}$ & 381,6 & 417,0 & 226,3 & 186,4 \\
\hline$m$ & \multicolumn{2}{|c|}{199} & \multicolumn{2}{|c|}{154} \\
\hline$\hat{t}\left(\right.$ für $\left.\mathrm{v}_{50}\right)$ & \multicolumn{2}{|c|}{2,34} & \multicolumn{2}{|c|}{0,30} \\
\hline$t($ für $\otimes=0,05)$ & \multicolumn{2}{|c|}{1,66} & \multicolumn{2}{|c|}{1,66} \\
\hline $\begin{array}{l}\text { Unterschied } \\
\text { signifikant? }\end{array}$ & \multicolumn{2}{|c|}{ ja } & \multicolumn{2}{|c|}{ nein } \\
\hline
\end{tabular}




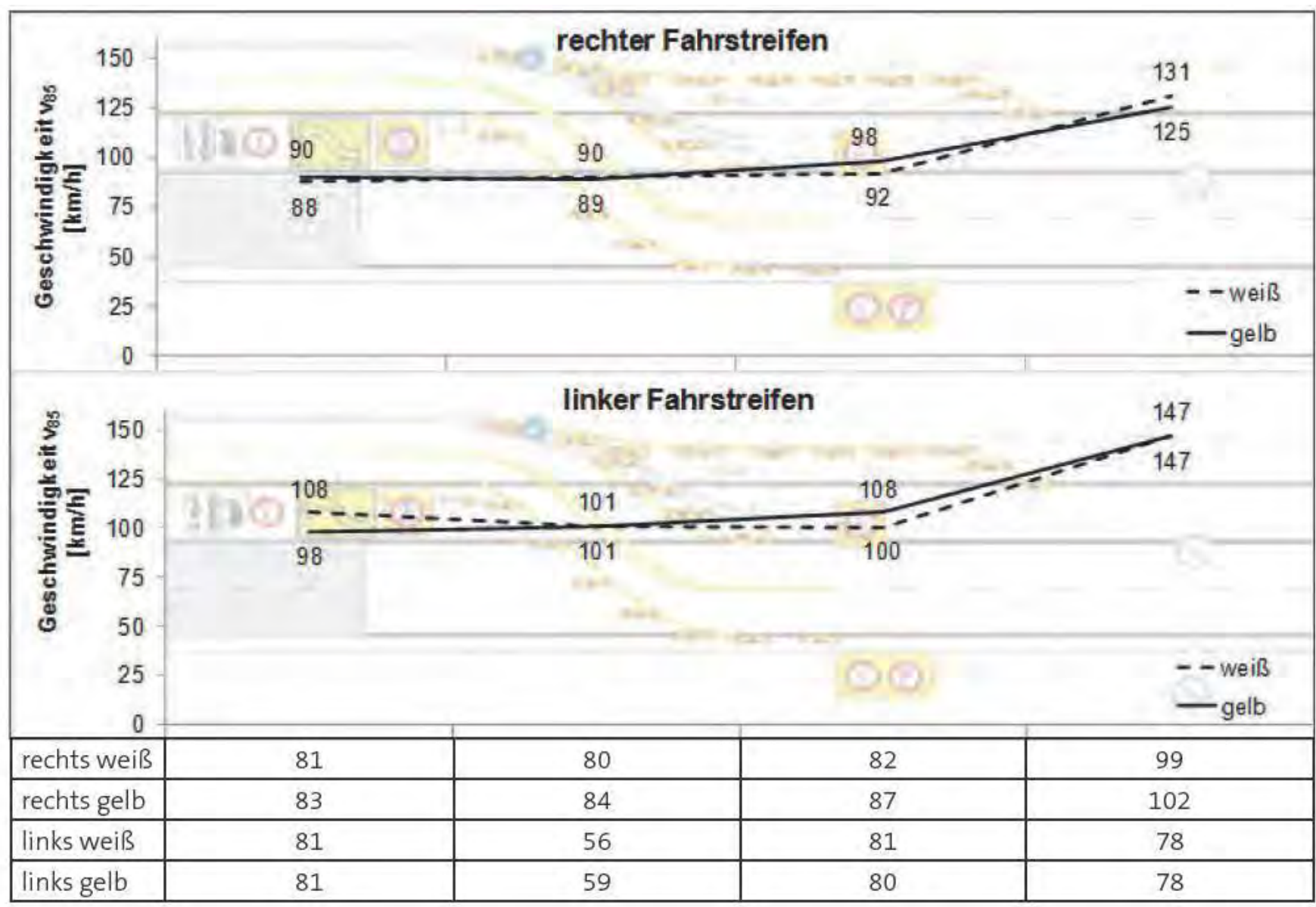

Abbildung D-9:

Geschwindigkeiten $\mathbf{v}_{85}$ am Baustellenende der Fahrtrichtung 1 nach Fahrstreifen mit dem zu Grunde liegenden Untersuchungskollektiv (Tabelle)

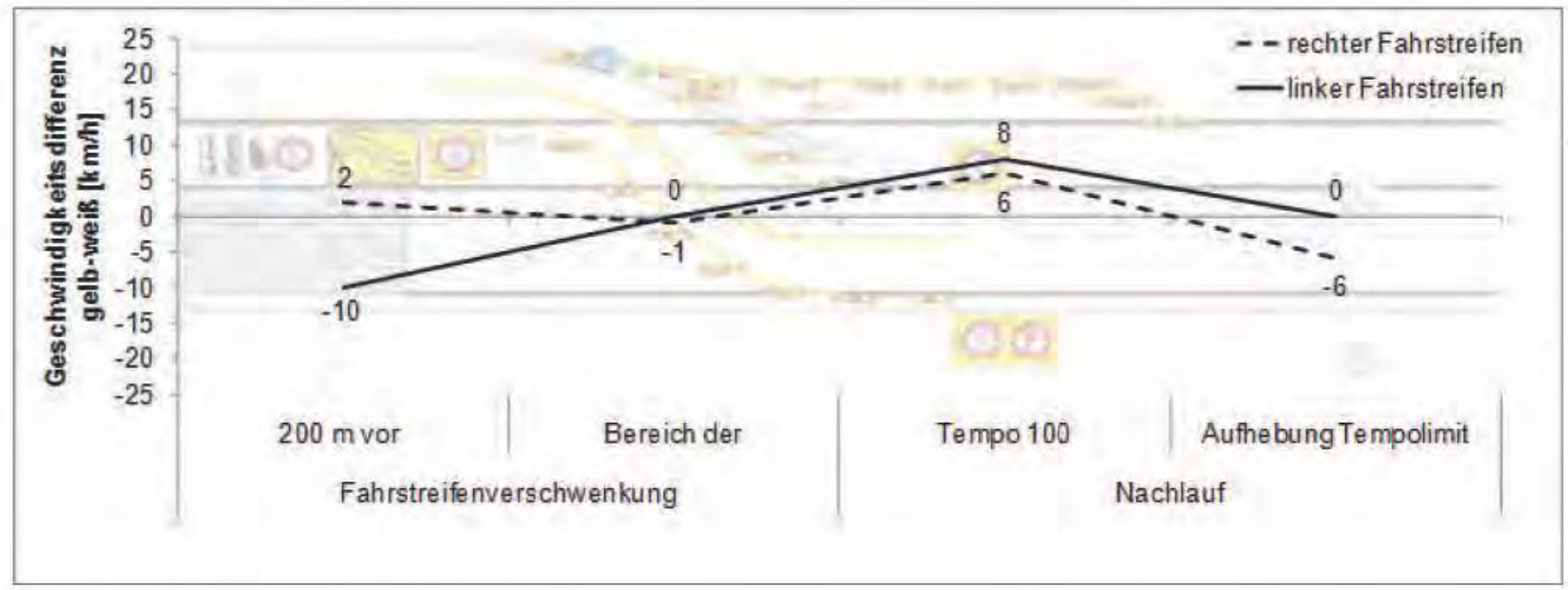

Abbildung D-10:

Differenz der Geschwindigkeiten $\mathbf{v}_{85}$ der Untersuchungsphasen am Baustellende der Fahrtrichtung 1 nach Fahrstreifen 


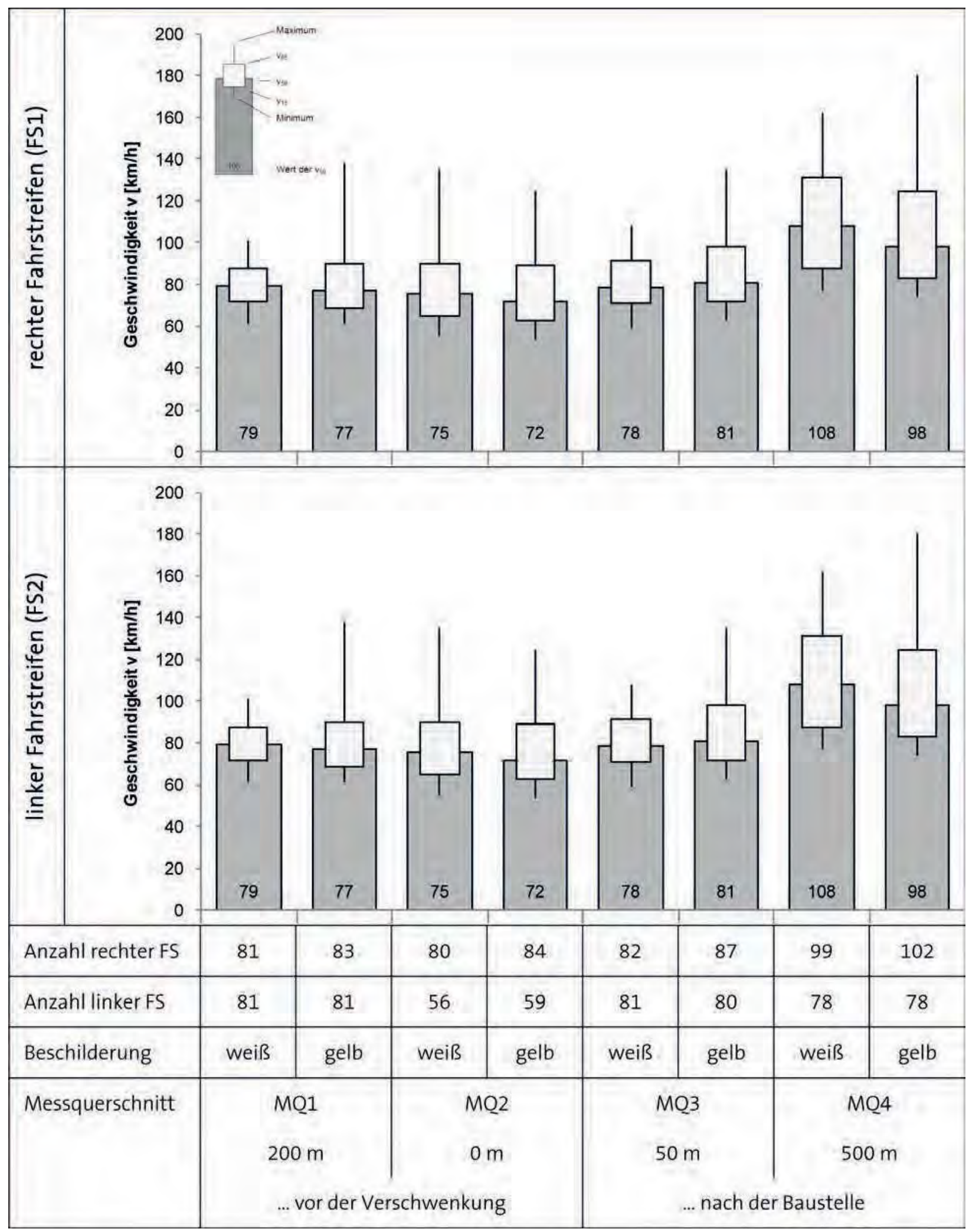

Abbildung D 11:

Kenngrößen der Geschwindigkeitsverteilung an den Messquerschnitten am Baustellenende der Fahrtrichtung 1 
Fahrtrichtung 2

Tabelle D-16:

Geschwindigkeitskenngrößen am Baustellende Fahrtrichtung 2 Querschnitt 1

\begin{tabular}{|c|c|c|c|c|}
\hline \multicolumn{5}{|c|}{$200 \mathrm{~m}$ vor der Fahrstreifenüberleitung } \\
\hline & \multicolumn{2}{|c|}{ Fahrstreifen 1} & \multicolumn{2}{|c|}{ Fahrstreifen 2} \\
\hline & weiß & gelb & weiß & gelb \\
\hline Verkehrsstärke q & 402 & 432 & 158 & 161 \\
\hline$v_{m}$ & 82 & 79 & 96 & 92 \\
\hline $\mathrm{V}_{85}$ & 92 & 85 & 108 & 101 \\
\hline Anzahl freier Fz & 78 & 75 & 79 & 74 \\
\hline$S^{2}$ & 89,87 & 55,58 & 155,48 & 79,37 \\
\hline $\mathrm{m}$ & \multicolumn{2}{|c|}{151} & \multicolumn{2}{|c|}{151} \\
\hline$\hat{t}\left(\right.$ für $\left.v_{50}\right)$ & \multicolumn{2}{|c|}{1,95} & \multicolumn{2}{|c|}{2,38} \\
\hline$t(f u ̈ r \otimes=0,05)$ & \multicolumn{2}{|c|}{1,66} & \multicolumn{2}{|c|}{1,66} \\
\hline $\begin{array}{l}\text { Unterschied } \\
\text { signifikant? }\end{array}$ & \multicolumn{2}{|c|}{ ja } & \multicolumn{2}{|c|}{ ja } \\
\hline
\end{tabular}

Tabelle D-17:

Geschwindigkeitskenngrößen am Baustellende Fahrtrichtung 2 Querschnitt 2

\begin{tabular}{|c|c|c|c|c|}
\hline \multicolumn{5}{|c|}{$0 \mathrm{~m}$ vor der Fahrstreifenüberleitung } \\
\hline & \multicolumn{2}{|c|}{ Fahrstreifen 1} & \multicolumn{2}{|c|}{ Fahrstreifen 2} \\
\hline & weiß & gelb & weiß & gelb \\
\hline Verkehrsstärke q & 404 & 483 & 191 & 157 \\
\hline$v_{m}$ & 84 & 74 & 95 & 84 \\
\hline $\mathrm{V}_{85}$ & 95 & 81 & 108 & 93 \\
\hline Anzahl freier Fz & 89 & 150 & 84 & 73 \\
\hline$S^{2}$ & 107,3 & 61,8 & 115,4 & 93,9 \\
\hline$m$ & \multicolumn{2}{|c|}{237} & \multicolumn{2}{|c|}{155} \\
\hline$\hat{t}\left(\right.$ für $\left.\mathrm{v}_{50}\right)$ & \multicolumn{2}{|c|}{8,85} & \multicolumn{2}{|c|}{6,51} \\
\hline$t($ für $\otimes=0,05)$ & \multicolumn{2}{|c|}{1,65} & \multicolumn{2}{|c|}{1,66} \\
\hline $\begin{array}{l}\text { Unterschied } \\
\text { signifikant? }\end{array}$ & \multicolumn{2}{|c|}{ ja } & \multicolumn{2}{|c|}{ ja } \\
\hline
\end{tabular}


Tabelle D-18:

Geschwindigkeitskenngrößen am Baustellende Fahrtrichtung 2 Querschnitt 3

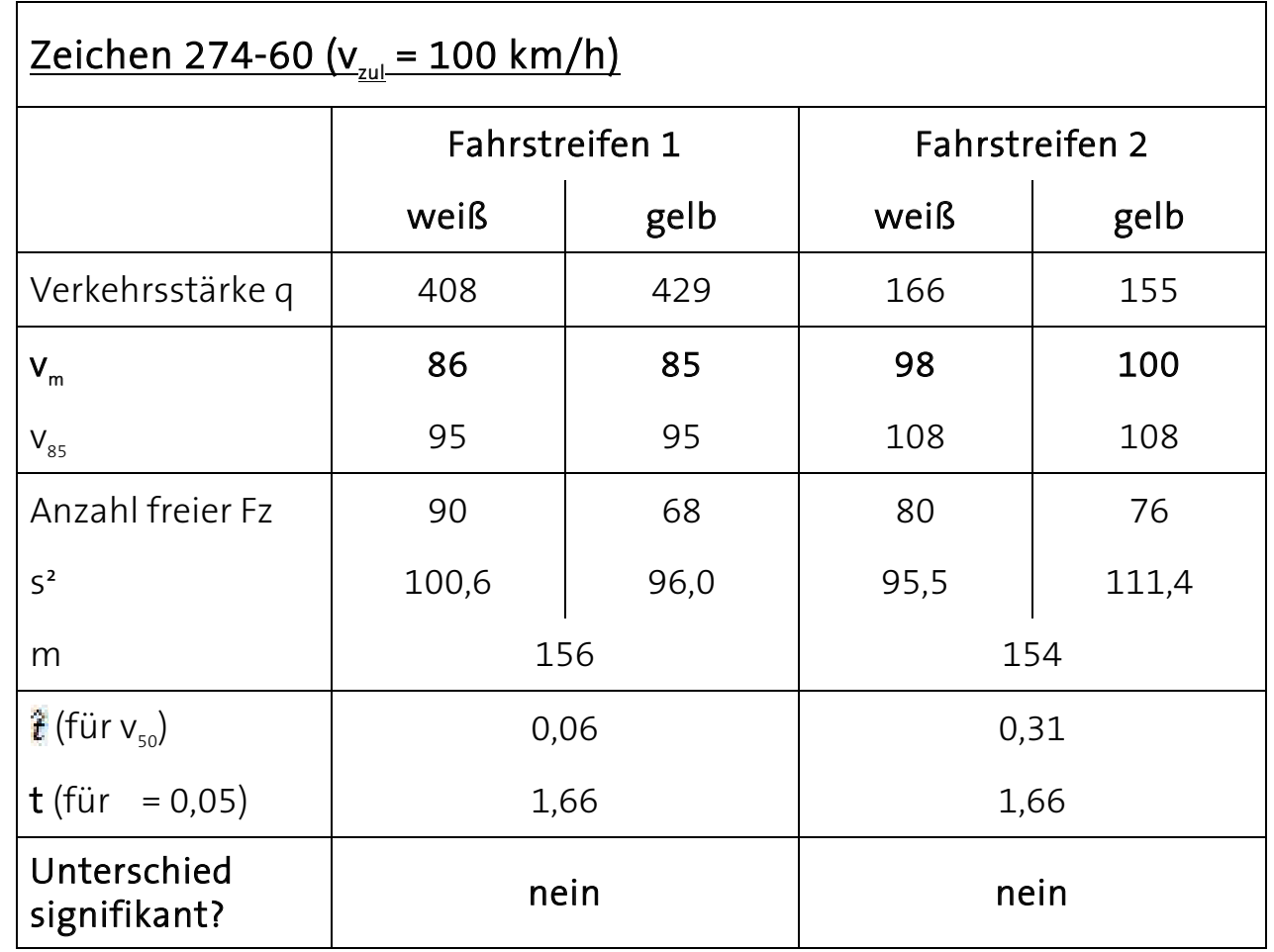

Tabelle D-19:

Geschwindigkeitskenngrößen am Baustellende Fahrtrichtung 2 Querschnitt 4

Zeichen 274-63 ( $\underline{\text { zul }}=130 \mathrm{~km} / \mathrm{h})$

\begin{tabular}{|c|c|c|c|c|}
\hline & \multicolumn{2}{|c|}{ Fahrstreifen 1} & \multicolumn{2}{|c|}{ Fahrstreifen 2} \\
\hline & weiß & gelb & weiß & gelb \\
\hline Verkehrsstärke q & 327 & 292 & 182 & 195 \\
\hline$v_{m}$ & 96 & 101 & 113 & 125 \\
\hline $\mathrm{v}_{85}$ & 108 & 116 & 125 & 135 \\
\hline Anzahl freier Fz & 99 & 101 & 66 & 75 \\
\hline$s^{2}$ & 193,13 & 249,49 & 145,89 & 188,71 \\
\hline$m$ & \multicolumn{2}{|c|}{198} & \multicolumn{2}{|c|}{139} \\
\hline$\hat{t}$ (für $v_{50}$ ) & \multicolumn{2}{|c|}{2,18} & \multicolumn{2}{|c|}{5,43} \\
\hline $\mathbf{t}$ (für $\alpha=0,05$ ) & \multicolumn{2}{|c|}{1,66} & \multicolumn{2}{|c|}{1,66} \\
\hline $\begin{array}{l}\text { Unterschied } \\
\text { signifikant? }\end{array}$ & \multicolumn{2}{|c|}{ ja } & \multicolumn{2}{|c|}{ ja } \\
\hline
\end{tabular}




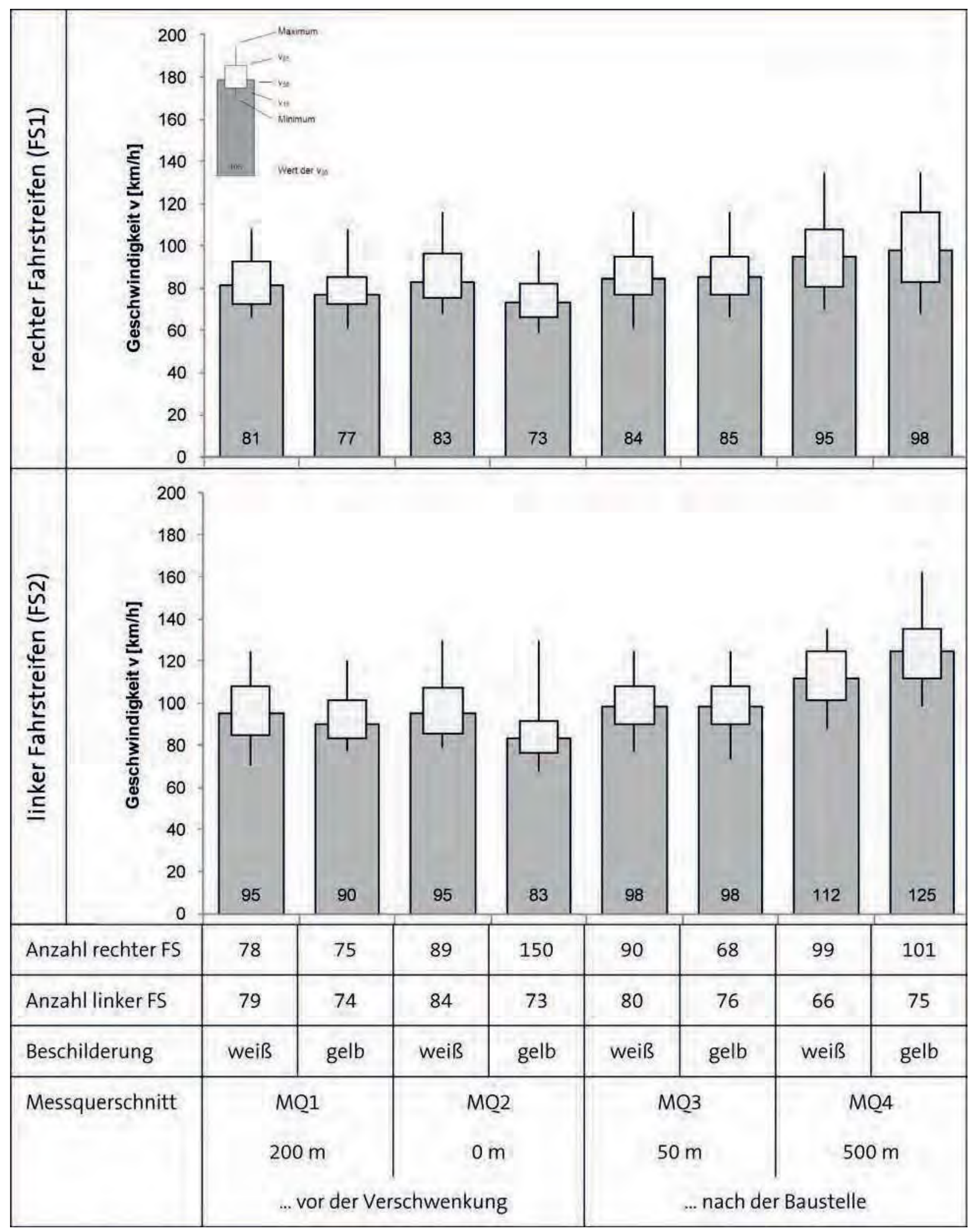

Abbildung D-12:

Kenngrößen der Geschwindigkeitsverteilung an den Messquerschnitten am Baustellenende der Fahrtrichtung 2 
D.2.4 Anschlussstelle

Tabelle D-20:

Geschwindigkeitskenngrößen vor Anschlussstelle Fahrtrichtung 2 Querschnitt 1

\section{$200 \mathrm{~m}$ vor Verzögerungsstreifen}

\begin{tabular}{|c|c|c|c|c|}
\hline & \multicolumn{2}{|c|}{ Fahrstreifen 1} & \multicolumn{2}{|c|}{ Fahrstreifen 2} \\
\hline & weiß & gelb & weiß & gelb \\
\hline Verkehrsstärke q & 602 & 374 & 161 & 101 \\
\hline $\mathrm{v}_{\mathrm{m}}$ & 84 & 90 & 97 & 96 \\
\hline $\mathrm{V}_{85}$ & 90 & 100 & 108 & 101 \\
\hline Anzahl freier Fz & 126 & 63 & 46 & 35 \\
\hline$s^{2}$ & 96,7 & 93,9 & 161,4 & 94,4 \\
\hline $\mathrm{m}$ & \multicolumn{2}{|c|}{187} & \multicolumn{2}{|c|}{79} \\
\hline$\hat{t}\left(\right.$ für $\mathrm{v}_{50}$ ) & \multicolumn{2}{|c|}{4,44} & \multicolumn{2}{|c|}{0,54} \\
\hline$t(f u ̈ r \otimes=0,05)$ & \multicolumn{2}{|c|}{1,66} & \multicolumn{2}{|c|}{1,68} \\
\hline $\begin{array}{l}\text { Unterschied } \\
\text { signifikant? }\end{array}$ & \multicolumn{2}{|c|}{ ja } & \multicolumn{2}{|c|}{ nein } \\
\hline
\end{tabular}

Tabelle D-21:

Geschwindigkeitskenngrößen vor Anschlussstelle Fahrtrichtung 2 Querschnitt 2

\begin{tabular}{|c|c|c|c|c|}
\hline \multicolumn{5}{|c|}{$0 \mathrm{~m}$ vor Verzögerungsstreifen } \\
\hline & \multicolumn{2}{|c|}{ Fahrstreifen 1} & \multicolumn{2}{|c|}{ Fahrstreifen 2} \\
\hline & weiß & gelb & weiß & gelb \\
\hline Verkehrsstärke q & 594 & 440 & 154 & 156 \\
\hline$v_{m}$ & 81 & 87 & 96 & 100 \\
\hline $\mathrm{V}_{85}$ & 90 & 95 & 108 & 108 \\
\hline Anzahl freier Fz & 119 & 86 & 49 & 51 \\
\hline$s^{2}$ & 81,8 & 89,3 & 107,5 & 110,6 \\
\hline $\mathrm{m}$ & \multicolumn{2}{|c|}{203} & \multicolumn{2}{|c|}{98} \\
\hline$\hat{t}\left(\right.$ für $\left.v_{50}\right)$ & \multicolumn{2}{|c|}{4,75} & \multicolumn{2}{|c|}{1,77} \\
\hline$t($ für $\otimes=0,05)$ & \multicolumn{2}{|c|}{1,65} & \multicolumn{2}{|c|}{1,68} \\
\hline $\begin{array}{l}\text { Unterschied } \\
\text { signifikant? }\end{array}$ & \multicolumn{2}{|c|}{ ja } & \multicolumn{2}{|c|}{ ja } \\
\hline
\end{tabular}




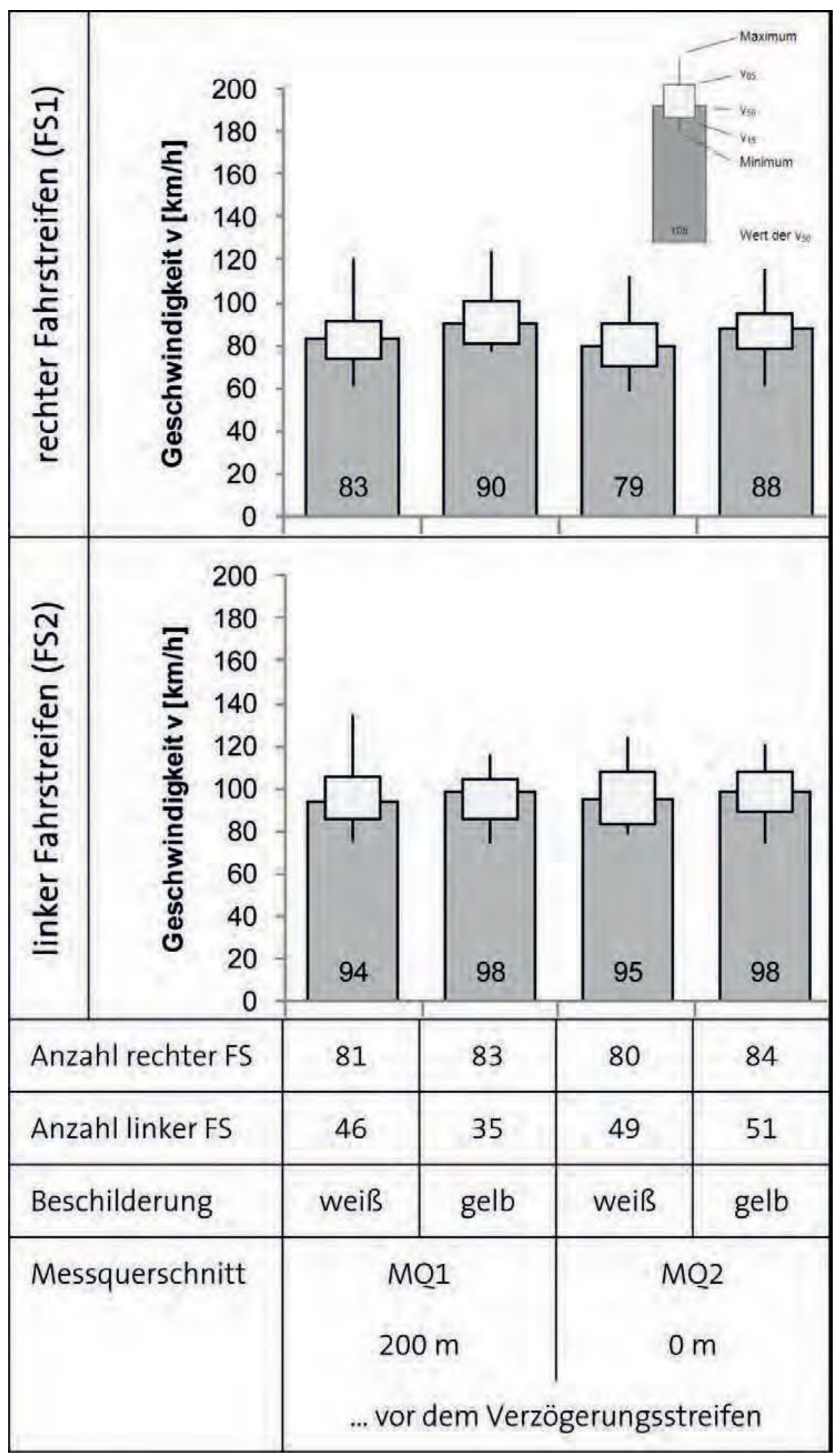




\section{E Längsabstände}

\section{E.1 Methodik}

Die Bewertung der Längsabstände zwischen hintereinander fahrenden Fahrzeugen basiert auf den Zeitlücken. Als kritisch werden die Zeitlücken angesehen, die eine Unterschreitung des Sicherheitsabstands darstellen. Dieser Sicherheitsabstand wird vereinfacht über die gebräuchliche Faustregel „Sicherheitsabstand = halber Tacho“ bestimmt. Demnach müssten Fahrzeuge bei 80 km/h einen Abstand von $40 \mathrm{~m}$ zum vorausfahrenden Fahrzeug einhalten. Der Sicherheitsabstand entspricht stets einer Zeitlücke von 1,8 s.

Die Verteilung der Größe der Längsabstände wird durch die Verkehrsdichte bestimmt und steht damit im Zusammenhang mit der Verkehrsstärke.

$$
\mathrm{D}=\frac{\mathrm{N}}{\Delta \mathrm{S}}=\frac{\mathrm{q}}{\mathrm{v}_{\mathrm{R}}}
$$

D Verkehrsdichte $[\mathrm{Fz} / \mathrm{km}]$

$\mathrm{N} \quad$ Anzahl der Verkehrselemente [Fz] auf einer Strecke

As Länge des Streckenabschnitts [km]

q Verkehrsstärke [Fz/h]

$v_{R} \quad$ Reisegeschwindigkeit $(\mathrm{km} / \mathrm{h}]$

Mit zunehmender Verkehrsstärke und -dichte steigt der Anteil kürzerer Zeitlücken zwischen den Fahrzeugen.
Für die Bewertung der Längsabstände wurde vereinfacht eine Betrachtung der Über- und Unterschreitung des Sicherheitsabstands (1,8 s) vorgenommen. Die Unterschreitung wurde zusätzlich in eine mittlere (oder einfache) Unterschreitung und eine starke Unterschreitung unterteilt (Tabelle E 1). Die mittlere Unterschreitung beschreibt dabei Längsabstände zwischen einer knappen Unterschreitung (0,01 s) und der Hälfte des erforderlichen Sicherheitsabstands (0,9 s = $50 \%)$. Eine starke Unterschreitung liegt dann vor, wenn das betrachtete Fahrzeug mit einer Zeitlücke unter 0,9 s seinem Vordermann folgt.

\section{Beispiel}

Bei einer Geschwindigkeit, im Bereich der zulässigen Höchstgeschwindigkeit in der Baustelle von $80 \mathrm{~km} / \mathrm{h}$ würde eine „mittlere Unterschreitung des Sicherheitsabstands" einer Nettoraumlücke zwischen 20 m und 39,9 $\mathrm{m}$ und eine „starke Unterschreitung" einem Abstand von weniger als $20 \mathrm{~m}$ entsprechen (Abbildung E-1).

Für die Analyse der Einhaltung der Sicherheitsabstände wurden die fahrstreifenbezogenen Nettozeitlücken zwischen den Fahrzeugen ausgewertet. Es zeigt sich, dass mit der Zunahme der Verkehrsstärken auch der Anteil der Unterschreitungen des Sicherheitsabstands zunimmt (Abbildung E-2). Dieser Effekt wirkt sich insbesondere auf die Abstände der Fahrzeuge auf dem meist höher belasteten rechten Fahrstreifen aus. In Baustellen fahren i. d. R. weniger als 35 \% auf dem linken Fahrstreifen (Abbildung E-3).

Tabelle E-1:

Unterteilung der Längsabstände

\begin{tabular}{|l|c|c|}
\hline Bezeichnung & $\begin{array}{c}\text { Anteil am } \\
\text { Sicherheitsabstand } \\
1,8 \mathrm{~s} \mathrm{zu} \mathrm{...}\end{array}$ & Längsabstand \\
\hline Einhaltung des Sicherheitsabstands & $\geq 100 \%$ & $\geq 1,8 \mathrm{~s}$ \\
\hline Mittlere Unterschreitung & $50 \% \ldots 99 \%$ & $0,9 \mathrm{~s} \ldots 1,79 \mathrm{~s}$ \\
\hline \begin{tabular}{l} 
Starke Unterschreitung \\
\hline
\end{tabular} & $<50 \%$ & $<0,9 \mathrm{~s}$ \\
\hline
\end{tabular}




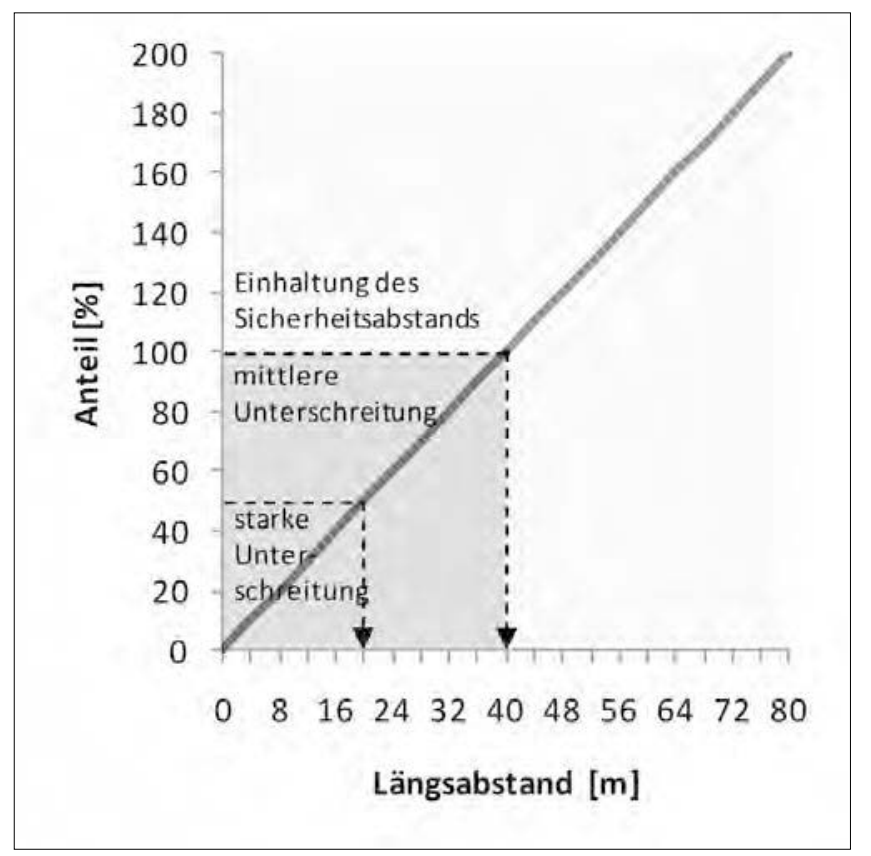

Im Folgenden sind die Häufigkeitsverteilungen der Einhaltungsgrade des Sicherheitsabstands für die verschiedenen Messquerschnitte dargestellt. Diese vergleichen die Ergebnisse für die rechten und linken Fahrstreifen des weißen und gelben Zustands.

Da insbesondere die Anteilswerte der Unterschreitung des Sicherheitsabstands bewertet werden sollen, ist lediglich der Bereich $0 \%$ bis $200 \%$ (x-Achse) dargestellt. Insbesondere für die Überholfahrstreifen würde sich bei Darstellung der gesamten Summenhäufigkeitslinie ein sehr großer Betrachtungsbereich ergeben. Hier treten häufiger Nettozeitlücken auf, die ein Vielfaches des Sicherheitsabstandes von $1,8 \mathrm{~s}$ bis hin zu einer Minute (>3.000\% des Sicherheitsabstandes) lang sein können.

Abbildung E-1:

Differenzierung der Einhaltung bzw. Unterschreitung des

Sicherheitsabstands am Beispiel $v=80 \mathrm{~km} / \mathrm{h}$

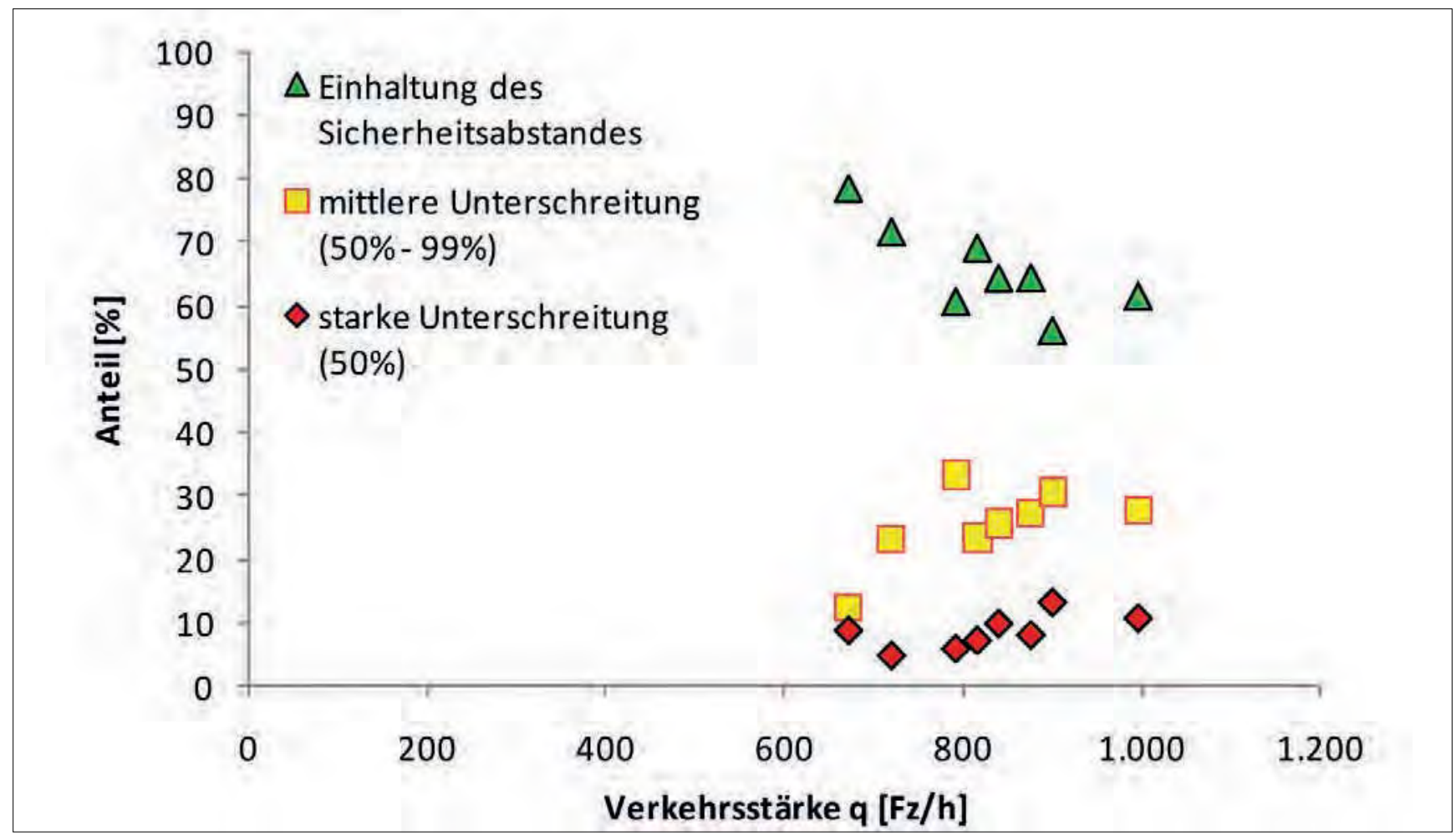

Abbildung E-2:

durchschnittliche Einhaltung der Sicherheitsabstände in Abhängigkeit der Verkehrsstärken vor der Verschwenkung am Baustellenbeginn (rechter Fahrstreifen, Beschilderung nach RSA - weiß) 


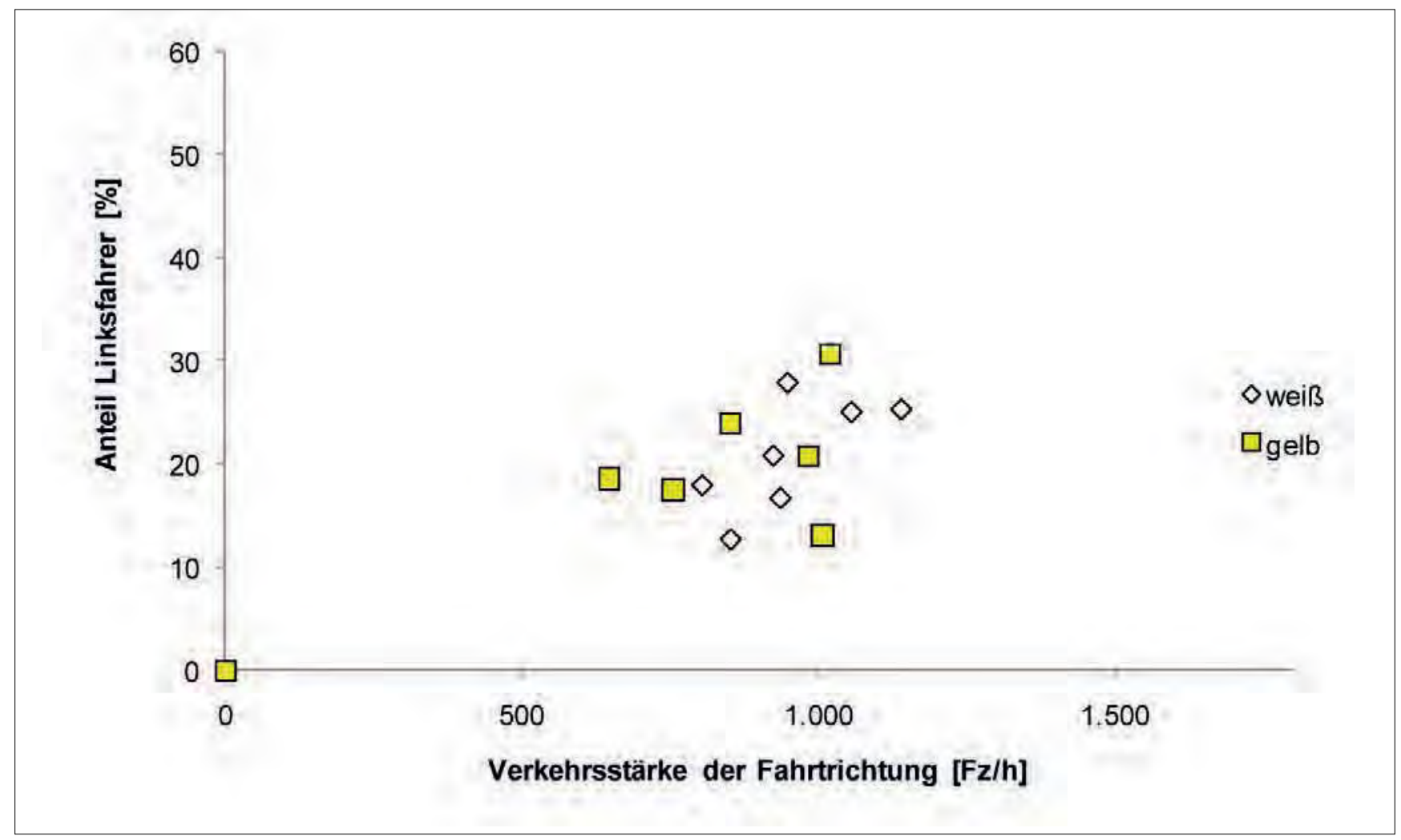

Abbildung E-3:

Anteil Linksfahrer in Abhängigkeit der Verkehrsstärken im Baustellenbereich 


\section{E.2 Auswertung}

Die Auswertung der Längsabstände erfolgte querschnittsbezogen für jeden Fahrstreifen separat. Dabei wurden die Ergebnisse der beiden betrachteten Beschilderungszustände (weiß und gelb) gegenübergestellt.

Gegenstand der Gegenüberstellung ist der in Abbildung E-1 beschriebene Anteil am Sicherheitsabstand. Damit bedeuten Werte von 99,9 (\%) und darunter, dass der Sicherheitsabstand unterschritten wurde.

Die sehr großen Zahlen, welche vorrangig auf dem linken (Überhol-)Fahrstreifen auftraten, resultieren aus großen Abständen zwischen den Fahrzeugen. Ein Wert von 1.000 [\%] beschreibt einen Längsabstand von etwa 18,2 s. Das entspricht bei einer Geschwindigkeit von $80 \mathrm{~km} / \mathrm{h}$ einem Längsabstand über $400 \mathrm{~m}$. Dieser ist $10 \mathrm{mal}$ so groß, wie der erforderliche Sicherheitsabstand bei dieser Geschwindigkeit $(40 \mathrm{~m})$.

Ziel dieser Betrachtung war die Identifizierung des Einflusses der modifizierten Beschilderung auf die von den Fahrern gewählten Abstände zum vorausfahrenden Fahrzeug. Neben den minimalen und den maximalen beobachteten Längsabständen wurden deshalb auch die Mittelwerte zunächst für das jeweilige Gesamtkollektiv des betrachteten Fahrstreifens und die Beschilderungsform dargestellt. Die Unterschiede zwischen den Ergebnissen eines Fahrstreifens für den weißen und den gelben Zustand wurden mit Hilfe von t-Tests auf Signifikanz überprüft.

Für die Betrachtung der als kritisch anzusehenden Längsabstände unterhalb des Sicherheitsabstandes wurde für diese eine gesonderte Betrachtung durchgeführt. Dabei wurde zunächst der Anteil der Abstände < 100(\%) an allen Zeitlücken sowie der mittlere Grad der Unterschreitung (Mittelwert [\%]) bestimmt. Der mittlere Grad der Unterschreitung der Sicherheitsabstände wurde wieder durch Parametertests (t-Tests) hinsichtlich seiner Signifikanz überprüft.

Alle Signifikanztests wurden für ein Signifikanzniveau von $\alpha=0,05$ durchgeführt.
Zuletzt wurden die Werte der mittleren Unterschreitung des Sicherheitsabstands, welche als Anteilswert vorlagen, in eine überschlägige Nettoraumlücke umgerechnet. Grundlage war eine angenommene Geschwindigkeit der Fahrzeuge von 80 km/h.

\section{$\underline{\text { Beispiel: }}$}

mittlere Unterschreitung des Sicherheitsabstands: $66(\%)$

angenommene Geschwindigkeit v: $\quad 80 \mathrm{~km} / \mathrm{h}$ erforderlicher Sicherheitsabstand: $\quad 40 \mathrm{~m}$

Die Unterschreitung des Sicherheitsabstands beträgt

$$
\mathrm{S}_{\mathrm{RL}}=\frac{40 \mathrm{~m} \cdot 66 \%}{100 \%}=\underline{\underline{26,40 \mathrm{~m}}}
$$

durchschnittlich 13,60 m. Der mittlere Abstand zum vorausfahrenden Fahrzeug beträgt in Fällen von zu geringem Sicherheitsabstand im Mittel 26,40 m.

Für die folgenden Tabellen und Abbildungen gelten die Abkürzungen:

Min Minimaler Anteil des Sicherheitsabstandes [\%]

Max Maximaler Anteil des Sicherheitsabstandes [\%]

$\mathrm{s}^{2} \quad$ Streuung

$m \quad$ Freiheitsgrade $\quad m=\left(n_{1}-1\right)+\left(n_{2}-1\right)$

$t^{*} \quad$ kritischer $t$-Wert (berechnet)

t Tabellenwert der Verteilung (Vergleichsgröße)

$\mathrm{S}_{\mathrm{RL}} \quad$ Nettoraumlücke [m]

FR Fahrtrichtung 


\section{E.3 Baustellenbeginn}

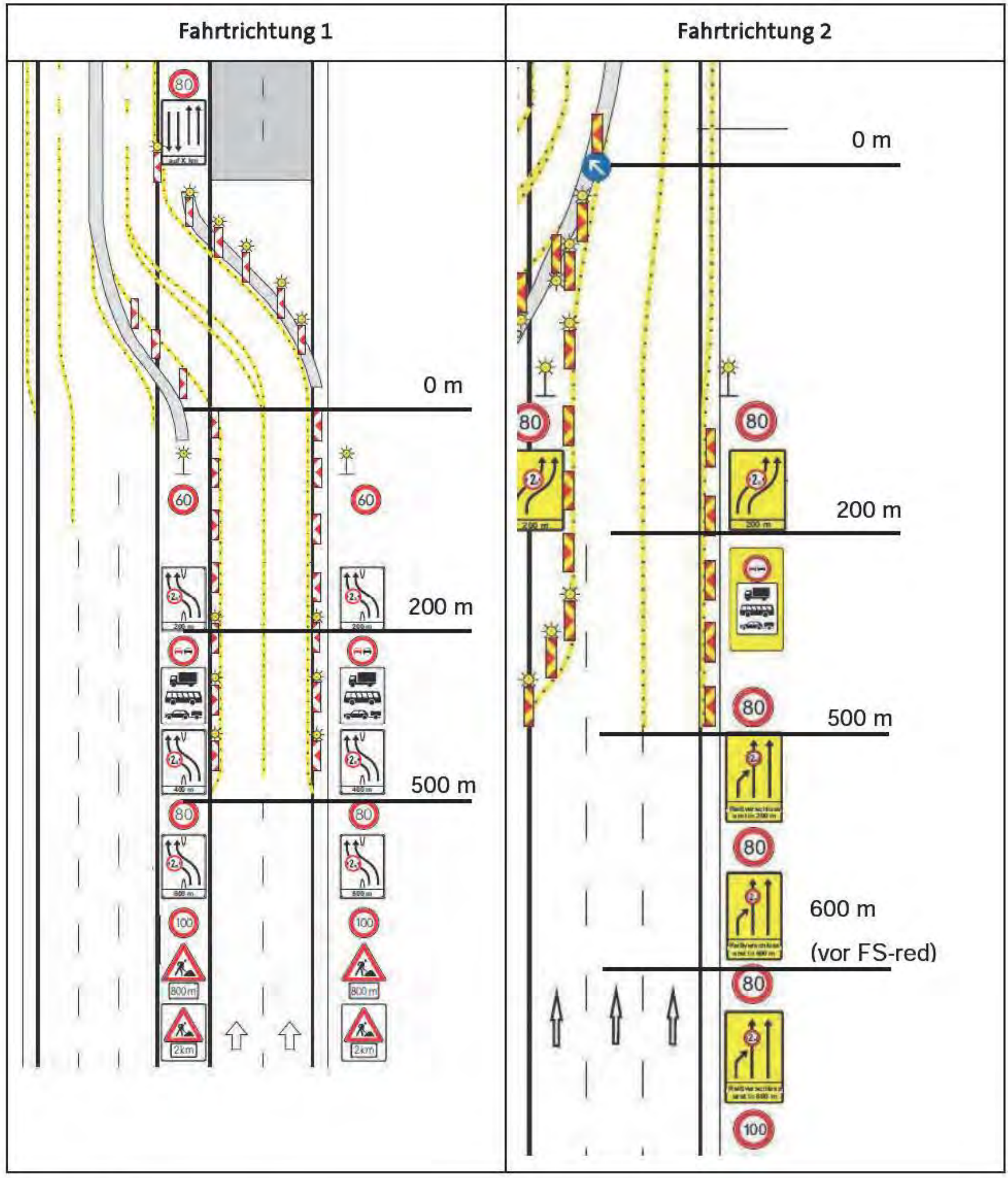

Abbildung E4:

Messquerschnitte Baustellenbeginn 


\section{Baustellenbeginn -}

$600 \mathrm{~m}$ vor der Fahrstreifenreduzierung (nur Fahrtrichtung 2)

\begin{tabular}{|c|c|c|c|c|c|c|}
\hline & \multicolumn{2}{|c|}{ Fahrtrichtung 1} & \multicolumn{4}{|c|}{ Fahrtrichtung 2} \\
\hline & FS1 & FS2 & \multicolumn{2}{|c|}{ FS1 } & \multicolumn{2}{|c|}{ FS2 } \\
\hline & weiß & weiß & weiß & gelb & weiß & gelb \\
\hline \multicolumn{7}{|c|}{ alle Zeitlücken (in \% des Sicherheitsabstandes I = 50\% der gefahrenen Geschwindigkeit) } \\
\hline $\operatorname{Min}[\%]$ & & & 26 & 11 & 17 & 27 \\
\hline $\operatorname{Max}[\%]$ & & & 1.710 & 1.373 & 3.192 & 2.631 \\
\hline $\begin{array}{l}\text { Mittelwert } \\
{[\%]}\end{array}$ & & & 298,6 & 316,2 & 495,0 & 488,3 \\
\hline Anzahl & & & 307 & 287 & 188 & 163 \\
\hline$s^{2}$ & & & 63.529 & 53.178 & 269.007 & 270.643 \\
\hline $\mathrm{m}$ & & & \multicolumn{2}{|c|}{592} & \multicolumn{2}{|c|}{349} \\
\hline$t^{*}$ & & & \multicolumn{2}{|c|}{0,88} & \multicolumn{2}{|c|}{0,12} \\
\hline t & & & \multicolumn{2}{|c|}{1,65} & \multicolumn{2}{|c|}{1,65} \\
\hline $\begin{array}{l}\text { Unterschied } \\
\text { signifikant? }\end{array}$ & & & \multicolumn{2}{|c|}{ nein } & \multicolumn{2}{|c|}{ nein } \\
\hline \multicolumn{7}{|c|}{ nur Unterschreitungen (in \% des Sicherheitsabstandes I = 50\% der gefahrenen Geschwindigkeit) } \\
\hline $\begin{array}{l}\text { Anteil an allen } \\
\text { Lücken [\%] }\end{array}$ & & & 23 & 14 & 17 & 20 \\
\hline $\begin{array}{l}\text { Mittelwert } \\
{[\%]}\end{array}$ & & & 65,8 & 71,5 & 62,7 & 68,1 \\
\hline Anzahl & & & 71 & 41 & 31 & 32 \\
\hline$s^{2}$ & & & 408,4 & 456,8 & 556,5 & 422,7 \\
\hline $\mathrm{m}$ & & & \multicolumn{2}{|c|}{110} & \multicolumn{2}{|c|}{61} \\
\hline$t^{*}$ & & & \multicolumn{2}{|c|}{1,41} & \multicolumn{2}{|c|}{0,96} \\
\hline t & & & \multicolumn{2}{|c|}{1,66} & \multicolumn{2}{|c|}{1,66} \\
\hline $\begin{array}{l}\text { Unterschied } \\
\text { signifikant? }\end{array}$ & & & \multicolumn{2}{|c|}{ nein } & \multicolumn{2}{|c|}{ nein } \\
\hline $\begin{array}{l}\mathrm{s}_{\mathrm{k}} \text { in }[\mathrm{m}] \\
\text { bei } 80 \mathrm{~km} / \mathrm{h}\end{array}$ & & & 26 & 29 & 25 & 27 \\
\hline
\end{tabular}

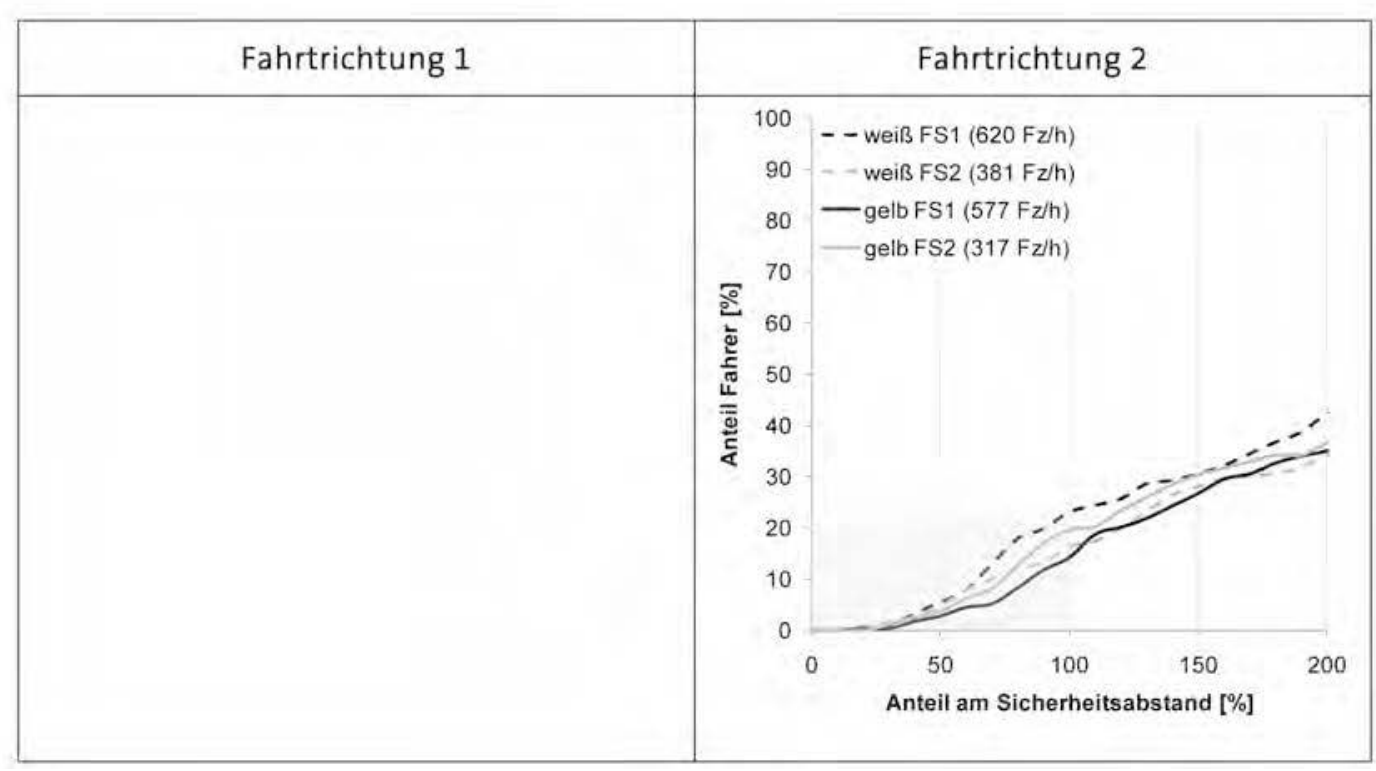




\section{Baustellenbeginn -}

$500 \mathrm{~m}$ vor der Fahrstreifenverschwenkung (Reduzierung der Fahrstreifen in FR 2)

\begin{tabular}{|c|c|c|c|c|c|c|c|c|}
\hline & \multicolumn{4}{|c|}{ Fahrtrichtung 1} & \multicolumn{4}{|c|}{ Fahrtrichtung 2} \\
\hline & \multicolumn{2}{|c|}{ FS1 } & \multicolumn{2}{|c|}{ FS2 } & \multicolumn{2}{|c|}{ FS1 } & \multicolumn{2}{|c|}{ F52 } \\
\hline & weiß & gelb & weiß & gelb & weiß & gelb & weiß & gelb \\
\hline \multicolumn{9}{|c|}{ alle Zeitlücken (in \% des Sicherheitsabstandes I = 50\% der gefahrenen Geschwindigkeit) } \\
\hline $\operatorname{Min}[\%]$ & 9 & 9 & 9 & 22 & 22 & 27 & 18 & 22 \\
\hline $\operatorname{Max}[\%]$ & 1,347 & 2.036 & 3.227 & 2.873 & 1.342 & 1.600 & 3.291 & 3.204 \\
\hline $\begin{array}{l}\text { Mittelwert } \\
{[\%]}\end{array}$ & 224,9 & 242,5 & 611,7 & 564,8 & 249,2 & 231,7 & 574,6 & 551,6 \\
\hline Anzahl & 404 & 382 & 131 & 105 & 357 & 398 & 178 & 170 \\
\hline$s^{2}$ & 41.665 & 50.961 & 482.431 & 487.472 & 54.893 & 38.793 & 425.981 & 388.640 \\
\hline $\mathrm{m}$ & \multicolumn{2}{|c|}{784} & \multicolumn{2}{|c|}{234} & \multicolumn{2}{|c|}{753} & \multicolumn{2}{|c|}{346} \\
\hline$t^{*}$ & \multicolumn{2}{|c|}{1,15} & \multicolumn{2}{|c|}{0,51} & \multicolumn{2}{|c|}{1,12} & \multicolumn{2}{|c|}{0,34} \\
\hline $\mathrm{t}$ & \multicolumn{2}{|c|}{1,65} & \multicolumn{2}{|c|}{1,65} & \multicolumn{2}{|c|}{1,65} & \multicolumn{2}{|c|}{1,65} \\
\hline $\begin{array}{l}\text { Unterschied } \\
\text { signifikant? }\end{array}$ & \multicolumn{2}{|c|}{ nein } & \multicolumn{2}{|c|}{ nein } & \multicolumn{2}{|c|}{ nein } & \multicolumn{2}{|c|}{ nein } \\
\hline \multicolumn{9}{|c|}{ nur Unterschreitungen (in \% des Sicherheitsabstandes $1=50 \%$ der gefahrenen Geschwindigkeit) } \\
\hline $\begin{array}{l}\text { Anteil an allen } \\
\text { Lücken [\%] }\end{array}$ & 30 & 29 & 25 & 27 & 31 & 28 & 20 & 21 \\
\hline $\begin{array}{l}\text { Mittelwert } \\
\text { [\%] }\end{array}$ & 60,2 & 66,0 & 53,1 & 57,0 & 64,1 & 67,4 & 50,7 & 60,4 \\
\hline Anzahl & 120 & 107 & 33 & 28 & 120 & 113 & 37 & 37 \\
\hline $5^{2}$ & 507,8 & 389,9 & 689,4 & 447,8 & 569,4 & 327,1 & 593,0 & 512,2 \\
\hline $\mathrm{m}$ & \multicolumn{2}{|c|}{225} & \multicolumn{2}{|c|}{59} & \multicolumn{2}{|c|}{231} & \multicolumn{2}{|c|}{72} \\
\hline$t^{*}$ & \multicolumn{2}{|c|}{2,19} & \multicolumn{2}{|c|}{0,63} & \multicolumn{2}{|c|}{1,20} & \multicolumn{2}{|c|}{1,77} \\
\hline t & \multicolumn{2}{|c|}{1,65} & & & & & & \\
\hline $\begin{array}{l}\text { Unterschied } \\
\text { signifikant? }\end{array}$ & & & & & & & & \\
\hline $\begin{array}{l}s_{\mathrm{g}} \text { in }[\mathrm{m}] \\
\text { bei } 80 \mathrm{~km} / \mathrm{h}\end{array}$ & 24 & 26 & 21 & 23 & 26 & 27 & 21 & 24 \\
\hline
\end{tabular}

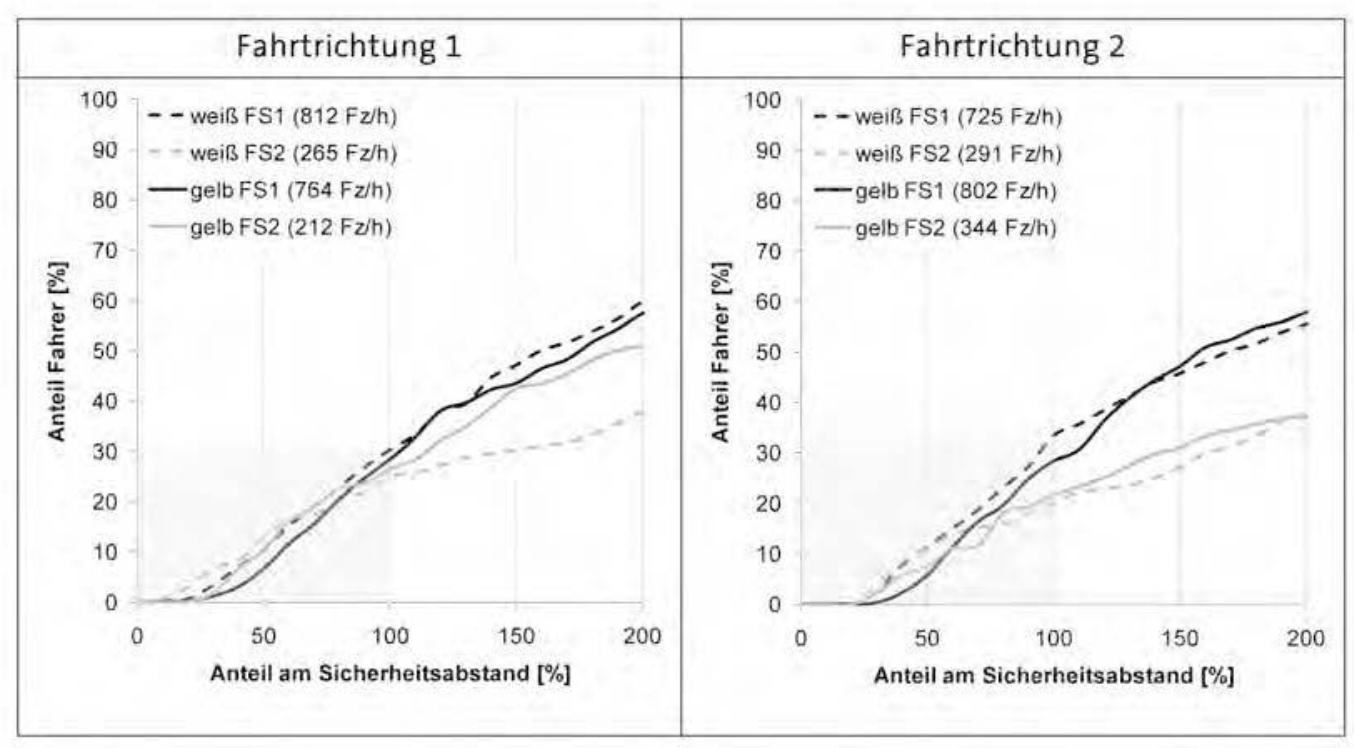




\section{Baustellenbeginn -}

$200 \mathrm{~m}$ vor der Fahrstreifenverschwenkung

\begin{tabular}{|c|c|c|c|c|c|c|c|c|}
\hline & \multicolumn{4}{|c|}{ Fahrtrichtung 1} & \multicolumn{4}{|c|}{ Fahrtrichtung 2} \\
\hline & \multicolumn{2}{|c|}{ FS1 } & \multicolumn{2}{|c|}{$\mathrm{F} 52$} & \multicolumn{2}{|c|}{ FS1 } & \multicolumn{2}{|c|}{ FS2 } \\
\hline & weiß & gelb & weiß & gelb & weiß & gelb & weiß & gelb \\
\hline \multicolumn{9}{|c|}{ alle Zeitlücken (in \% des Sicherheitsabstandes |= 50\% der gefahrenen Geschwindigkeit) } \\
\hline $\operatorname{Min}[\%]$ & 9 & 9 & 9 & 22 & 22 & 27 & 18 & 22 \\
\hline $\operatorname{Max}[\%]$ & 1.347 & 2.036 & 3.227 & 2.873 & 1.342 & 1.600 & 3.291 & 3.204 \\
\hline $\begin{array}{l}\text { Mittelwert } \\
{[\%]}\end{array}$ & 224,9 & 242,5 & 611,7 & 564,8 & 249,2 & 231,7 & 574,6 & 551,6 \\
\hline Anzahl & 404 & 382 & 131 & 105 & 357 & 398 & 178 & 170 \\
\hline$s^{2}$ & 41.665 & 50.961 & 482.431 & 487.472 & 54.893 & 38.793 & 425.981 & 388.640 \\
\hline $\mathrm{m}$ & \multicolumn{2}{|c|}{784} & \multicolumn{2}{|c|}{234} & \multicolumn{2}{|c|}{753} & \multicolumn{2}{|c|}{346} \\
\hline$t^{*}$ & \multicolumn{2}{|c|}{1,15} & \multicolumn{2}{|c|}{0,51} & \multicolumn{2}{|c|}{1.12} & \multicolumn{2}{|c|}{0,34} \\
\hline t & \multicolumn{2}{|c|}{1,65} & \multicolumn{2}{|c|}{1,65} & \multicolumn{2}{|c|}{1,65} & \multicolumn{2}{|c|}{1,65} \\
\hline $\begin{array}{l}\text { Unterschied } \\
\text { signifikant? }\end{array}$ & \multicolumn{2}{|c|}{ nein } & \multicolumn{2}{|c|}{ nein } & \multicolumn{2}{|c|}{ nein } & \multicolumn{2}{|c|}{ nein } \\
\hline \multicolumn{9}{|c|}{ nur Unterschreitungen (in \% des Sicherheitsabstandes I = 50\% der gefahrenen Geschwindigkeit) } \\
\hline $\begin{array}{l}\text { Anteil an allen } \\
\text { Lücken [\%] }\end{array}$ & 30 & 29 & 25 & 27 & 31 & 28 & 20 & 21 \\
\hline $\begin{array}{l}\text { Mittelwert } \\
{[\%]}\end{array}$ & 60,2 & 66,0 & 53,1 & 57,0 & 64,1 & 67,4 & 50,7 & 60,4 \\
\hline Anzahl & 120 & 107 & 33 & 28 & 120 & 113 & 37 & 37 \\
\hline$s^{2}$ & 507,8 & 389,9 & 689,4 & 447,8 & 569,4 & 327,1 & 593,0 & 512,2 \\
\hline$m$ & \multicolumn{2}{|c|}{225} & \multicolumn{2}{|c|}{59} & \multicolumn{2}{|c|}{231} & \multicolumn{2}{|c|}{72} \\
\hline$t^{*}$ & \multicolumn{2}{|c|}{2,19} & \multicolumn{2}{|c|}{0,63} & \multicolumn{2}{|c|}{1,20} & \multicolumn{2}{|c|}{1,77} \\
\hline t & \multicolumn{2}{|c|}{1,65} & & & & & & \\
\hline $\begin{array}{l}\text { Unterschied } \\
\text { signifikant? }\end{array}$ & & & & & & & & \\
\hline $\begin{array}{l}s_{s} \text { in }[\mathrm{m}] \\
\text { bei } 80 \mathrm{~km} / \mathrm{h}\end{array}$ & 24 & 26 & 21 & 23 & 26 & 27 & 21 & 24 \\
\hline
\end{tabular}

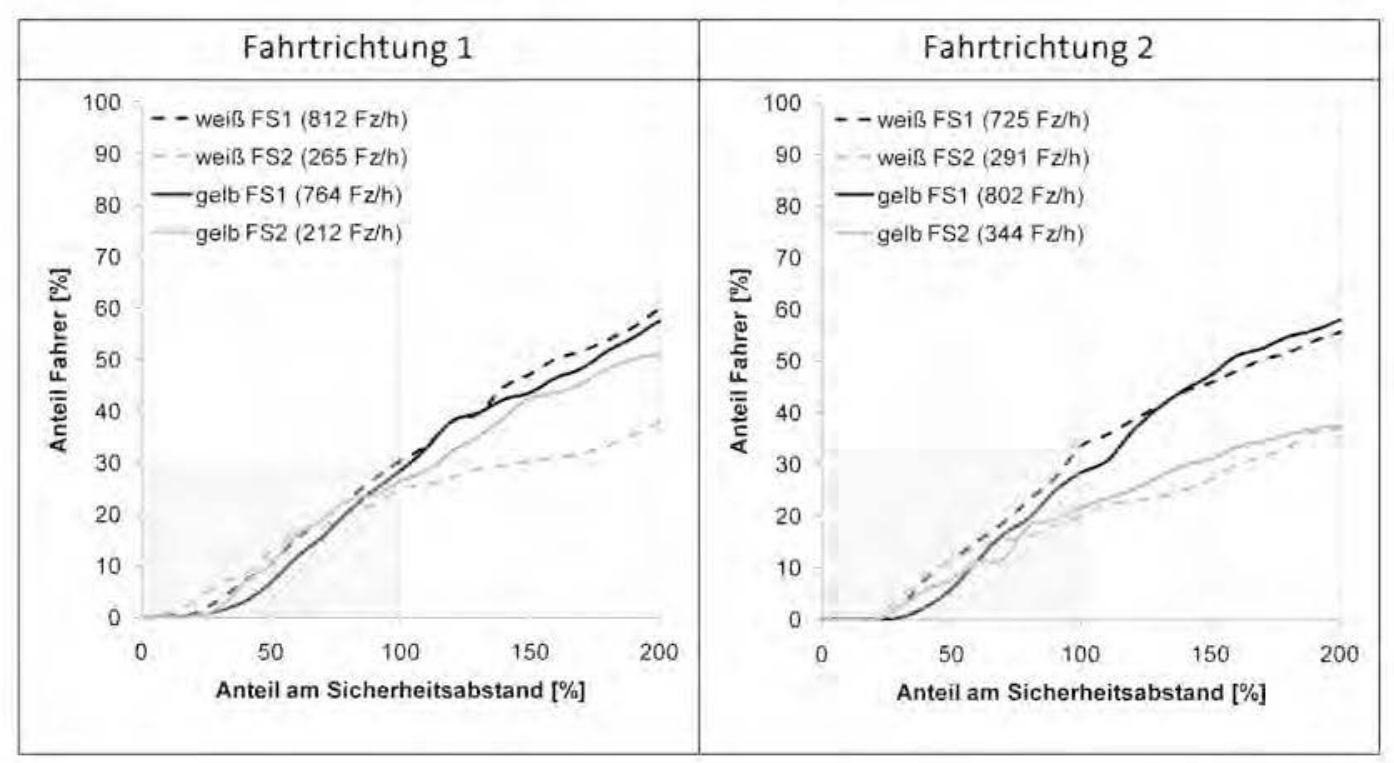




\section{Baustellenbeginn -}

Am Beginn der Fahrstreifenverschwenkung

\begin{tabular}{|c|c|c|c|c|c|c|c|c|}
\hline & \multicolumn{4}{|c|}{ Fahrtrichtung 1} & \multicolumn{4}{|c|}{ Fahrtrichtung 2} \\
\hline & \multicolumn{2}{|c|}{ FSI } & \multicolumn{2}{|c|}{ FS2 } & \multicolumn{2}{|c|}{ FS1 } & \multicolumn{2}{|c|}{ FS2 } \\
\hline & weiß & gelb & weiß & gelb & weiß & gelb & weiß & gelb \\
\hline \multicolumn{9}{|c|}{ alle Zeitlücken (in \% des Sicherheitsabstandes I = 50\% der gefahrenen Geschwindigkeit) } \\
\hline $\operatorname{Min}[\%]$ & 20 & 20 & 22 & 20 & 20 & 29 & 19 & 29 \\
\hline $\operatorname{Max}[\%]$ & 1.853 & 2.133 & 2.724 & 3.151 & $1.013,3$ & 1.373 & 2.683 & 2.967 \\
\hline $\begin{array}{l}\text { Mittelwert } \\
{[\%]}\end{array}$ & 223,6 & 227,9 & 577,3 & 632,7 & 207,8 & 239,6 & 389,3 & 535,4 \\
\hline Anzahl & 607 & 590 & 162 & 153 & 415 & 380 & 225 & 175 \\
\hline$s^{2}$ & 49.688 & 53.864 & 356.693 & 505.660 & 31.031 & 44.063 & 256.297 & 352.447 \\
\hline$m$ & \multicolumn{2}{|c|}{1.195} & \multicolumn{2}{|c|}{313} & \multicolumn{2}{|c|}{793} & \multicolumn{2}{|c|}{398} \\
\hline$t^{*}$ & \multicolumn{2}{|c|}{0,33} & \multicolumn{2}{|c|}{0.75} & \multicolumn{2}{|c|}{2,32} & \multicolumn{2}{|c|}{2,65} \\
\hline$t$ & \multicolumn{2}{|c|}{1,65} & \multicolumn{2}{|c|}{1,65} & \multicolumn{2}{|c|}{1,65} & \multicolumn{2}{|c|}{1,65} \\
\hline $\begin{array}{l}\text { Unterschied } \\
\text { signifikant? }\end{array}$ & \multicolumn{2}{|c|}{ nein } & \multicolumn{2}{|c|}{ nein } & \multicolumn{2}{|c|}{ ja } & \multicolumn{2}{|c|}{ ja } \\
\hline \multicolumn{9}{|c|}{ nur Unterschreitungen (in \% des Sicherheitsabstandes $1=50 \%$ der gefahrenen Geschwindigkeit) } \\
\hline $\begin{array}{l}\text { Anteil an allen } \\
\text { Lücken [\%] }\end{array}$ & 34 & 36 & 20 & 24 & 34 & 25 & 34 & 27 \\
\hline $\begin{array}{l}\text { Mittelwert } \\
{[\%]}\end{array}$ & 63,1 & 61,1 & 49,4 & 44,0 & 69,9 & 72,2 & 62,0 & 60,4 \\
\hline Anzahl & 218 & 223 & 33 & 36 & 142 & 95 & 77 & 48 \\
\hline$s^{2}$ & 362,1 & 400,9 & 323,5 & 306,8 & 371,5 & 311,1 & 375,0 & 400,7 \\
\hline$m$ & \multicolumn{2}{|c|}{439} & \multicolumn{2}{|c|}{67} & \multicolumn{2}{|c|}{235} & \multicolumn{2}{|c|}{123} \\
\hline$t^{*}$ & \multicolumn{2}{|c|}{1,09} & \multicolumn{2}{|c|}{1,28} & \multicolumn{2}{|c|}{0,94} & \multicolumn{2}{|c|}{0,43} \\
\hline $\mathrm{t}$ & \multicolumn{2}{|c|}{1,65} & & & & & & \\
\hline $\begin{array}{l}\text { Unterschied } \\
\text { signifikant? }\end{array}$ & & & & & & & & \\
\hline $\begin{array}{l}s_{n} \text { in }[\mathrm{m}] \\
\text { bei } 80 \mathrm{~km} / \mathrm{h}\end{array}$ & 25 & 24 & 20 & 18 & 28 & 29 & 25 & 24 \\
\hline
\end{tabular}

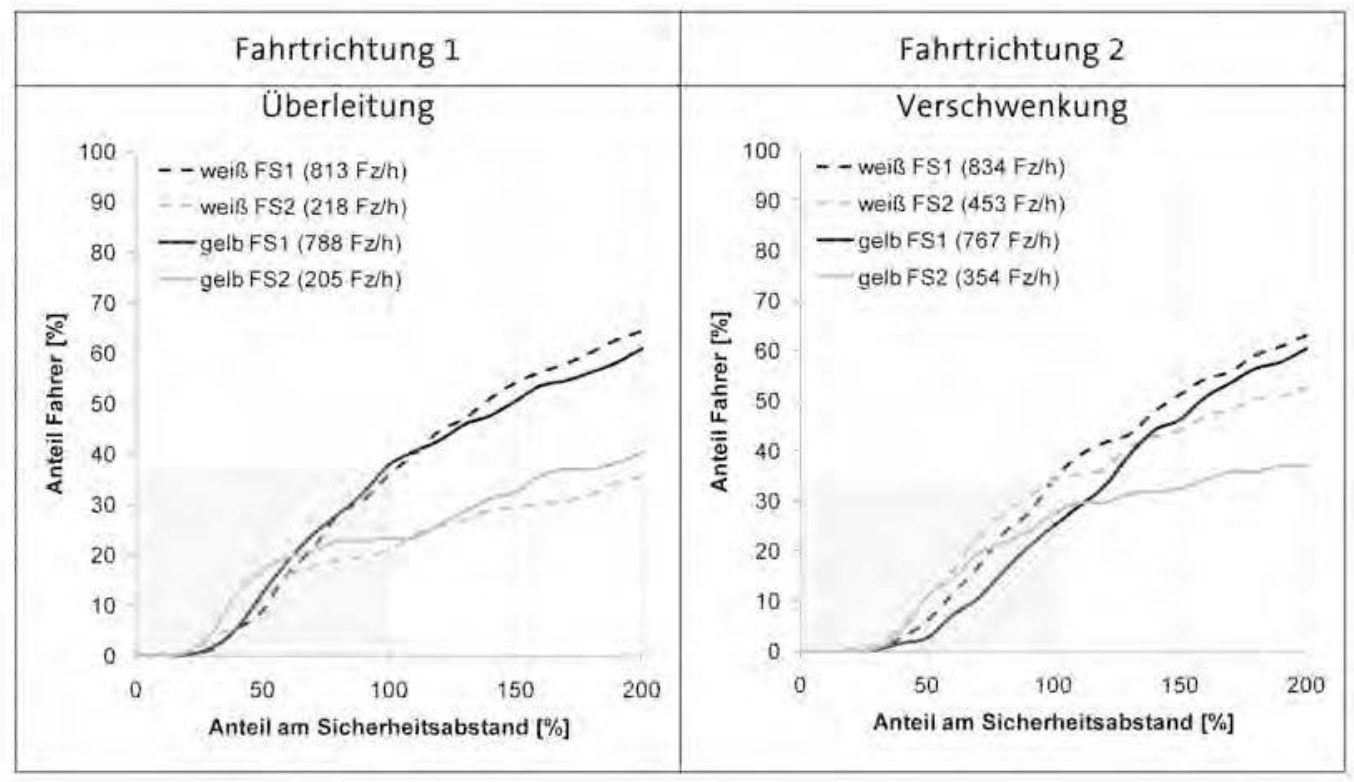


E.4 Baustellenende

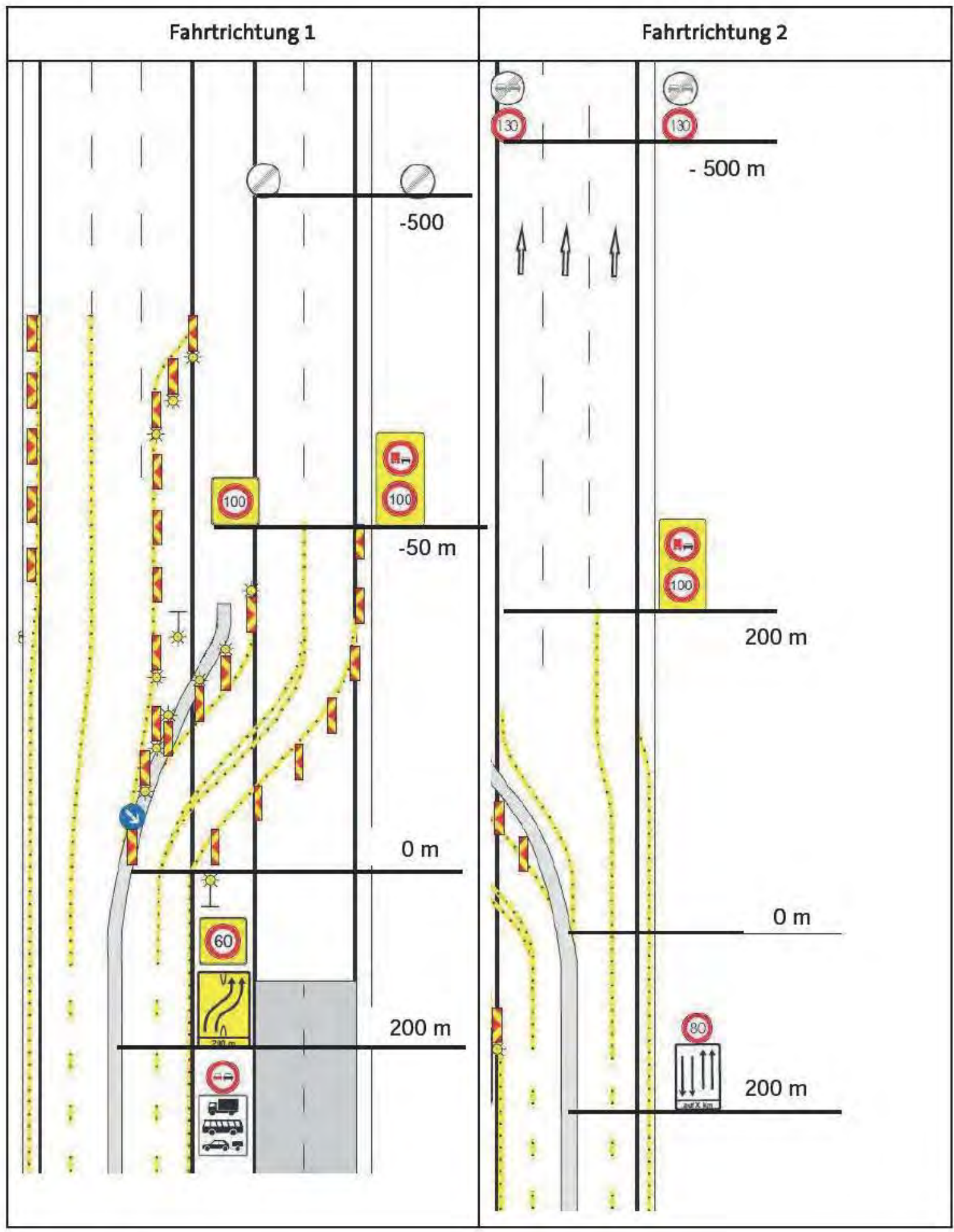

Abbildung E-5:

Messquerschnitte Baustellenbeginn 
Baustellenende -

$200 \mathrm{~m}$ vor der Fahrstreifenverschwenkung

\begin{tabular}{|c|c|c|c|c|c|c|c|c|}
\hline & \multicolumn{4}{|c|}{ Fahrtrichtung 1} & \multicolumn{4}{|c|}{ Fahrtrichtung 2} \\
\hline & \multicolumn{2}{|c|}{ FS1 } & \multicolumn{2}{|c|}{ FS2 } & \multicolumn{2}{|c|}{ FS1 } & \multicolumn{2}{|c|}{ FS2 } \\
\hline & weißs & gelb & weiß & gelb & weiß & gelb & weiß & gelb \\
\hline \multicolumn{9}{|c|}{ alle Zeitlücken (in \% des Sicherheitsabstandes $1=50 \%$ der gefahrenen Geschwindigkeit) } \\
\hline $\operatorname{Min}[\%]$ & 11 & 16 & 9 & 19 & 24 & 29 & 20 & 13 \\
\hline $\operatorname{Max}[\%]$ & 1.969 & 2.400 & 3.213 & 3.287 & 1,909 & 1.587 & 3.102 & 3.133 \\
\hline $\begin{array}{l}\text { Mittelwert } \\
{[\%]}\end{array}$ & 236,7 & 235,6 & 959,1 & 926,4 & 235,8 & 225,3 & 806,8 & 846,2 \\
\hline Anzahl & 380 & 393 & 143 & 141 & 386 & 370 & 152 & 145 \\
\hline$s^{2}$ & 95.088 & 83.015 & 865.869 & 857.434 & 74.165 & 52.859 & 738.030 & 687.328 \\
\hline$m$ & \multicolumn{2}{|c|}{771} & \multicolumn{2}{|c|}{282} & \multicolumn{2}{|c|}{754} & \multicolumn{2}{|c|}{295} \\
\hline $\mathrm{t}^{*}$ & \multicolumn{2}{|c|}{0,05} & \multicolumn{2}{|c|}{0,30} & \multicolumn{2}{|c|}{0,57} & \multicolumn{2}{|c|}{0,40} \\
\hline$t$ & \multicolumn{2}{|c|}{1,65} & \multicolumn{2}{|c|}{1,65} & \multicolumn{2}{|c|}{1,65} & \multicolumn{2}{|c|}{1,65} \\
\hline $\begin{array}{l}\text { Unterschied } \\
\text { signifikant? }\end{array}$ & \multicolumn{2}{|c|}{ nein } & \multicolumn{2}{|c|}{ nein } & \multicolumn{2}{|c|}{ nein } & \multicolumn{2}{|c|}{ nein } \\
\hline \multicolumn{9}{|c|}{ nur Unterschreitungen (in \% des Sicherheitsabstandes I = 50\% der gefahrenen Geschwindigkeit) } \\
\hline $\begin{array}{l}\text { Anteil an allen } \\
\text { Lücken [\%] }\end{array}$ & 47 & 47 & 20 & 22 & 40 & 38 & 25 & 23 \\
\hline $\begin{array}{l}\text { Mittelwert } \\
{[\%]}\end{array}$ & 57,0 & 61,9 & 46,8 & 54,1 & 61,9 & 63,7 & 51,1 & 50,2 \\
\hline Anzahl & 184 & 189 & 28 & 31 & 157 & 142 & 38 & 33 \\
\hline$s^{2}$ & 485 & 449 & 375 & 502 & 372 & 357 & 373 & 346 \\
\hline m & \multicolumn{2}{|c|}{371} & \multicolumn{2}{|c|}{57} & \multicolumn{2}{|c|}{297} & \multicolumn{2}{|c|}{69} \\
\hline$t^{*}$ & \multicolumn{2}{|c|}{2,19} & \multicolumn{2}{|c|}{1,33} & \multicolumn{2}{|c|}{0,82} & \multicolumn{2}{|c|}{0,18} \\
\hline $\mathrm{t}$ & \multicolumn{2}{|c|}{1,65} & & & & & & \\
\hline $\begin{array}{l}\text { Unterschied } \\
\text { signifikant? }\end{array}$ & & & & & & & & in \\
\hline $\begin{array}{l}s_{\mathrm{s}} \text { in }[\mathrm{m}] \\
\text { bei } 80 \mathrm{~km} / \mathrm{h}\end{array}$ & 23 & 25 & 19 & 22 & 25 & 25 & 20 & 20 \\
\hline
\end{tabular}

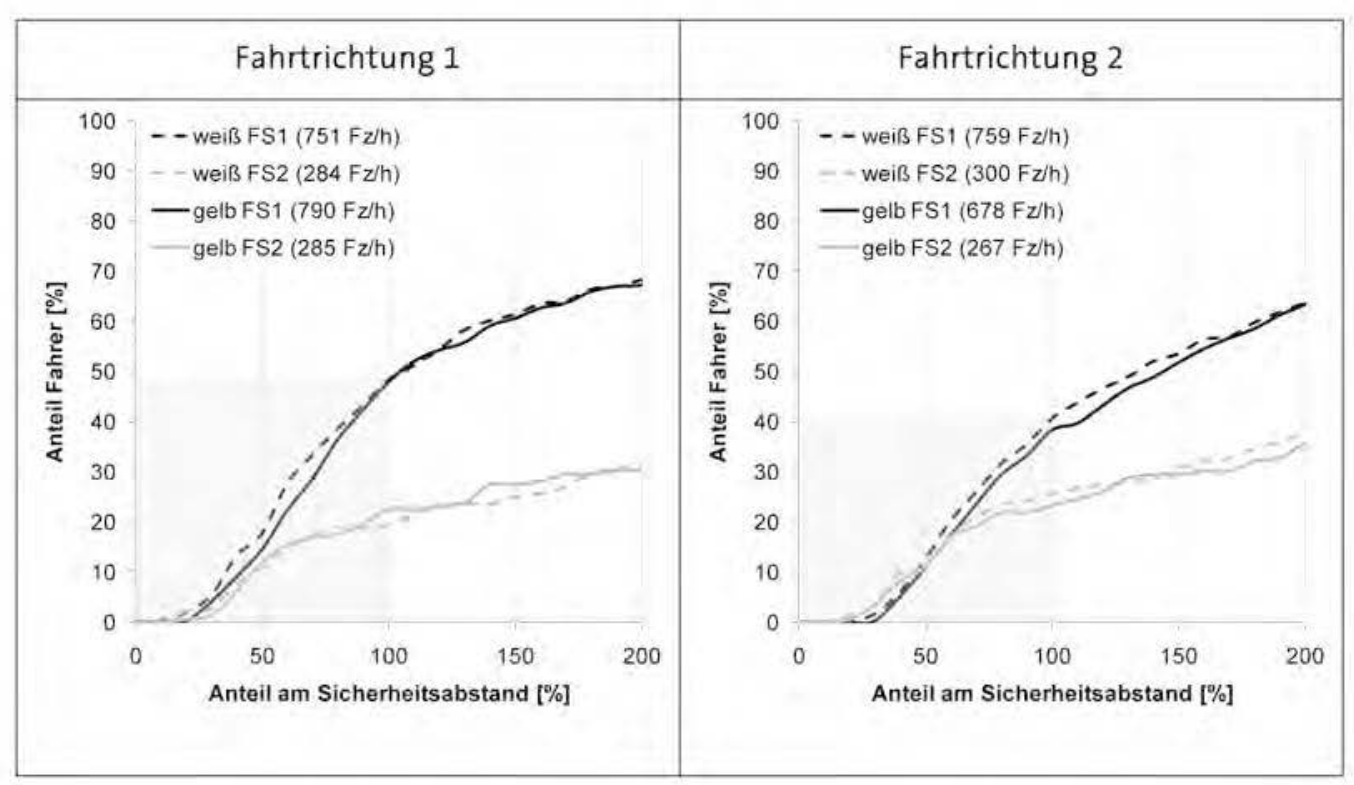




\section{Baustellenende -}

vor der Fahrstreifenverschwenkung

\begin{tabular}{|c|c|c|c|c|c|c|c|c|}
\hline & \multicolumn{4}{|c|}{ Fahrtrichtung 1} & \multicolumn{4}{|c|}{ Fahrtrichtung 2} \\
\hline & \multicolumn{2}{|c|}{ FS1 } & \multicolumn{2}{|c|}{ FS2 } & \multicolumn{2}{|c|}{ FS1 } & \multicolumn{2}{|c|}{ FS2 } \\
\hline & weiß & gelb & weiß & gelb & weiß & gelb & weiß & gelb \\
\hline \multicolumn{9}{|c|}{ alle Zeitlücken (in \% des Sicherheitsabstandes $1=50 \%$ der gefahrenen Geschwindigkeit) } \\
\hline $\operatorname{Min}[\%]$ & 16 & 24 & 20 & 24 & 24 & 24 & 16 & 22 \\
\hline $\operatorname{Max}[\%]$ & 1.193 & 2.524 & 2.689 & 3.071 & 1.893 & 1.403 & 3.233 & 3.296 \\
\hline $\begin{array}{l}\text { Mittelwert } \\
{[\%]}\end{array}$ & 202,4 & 233,7 & 523,5 & 755,9 & 243,7 & 252,6 & 906,9 & 788,2 \\
\hline Anzahl & 448 & 394 & 145 & 122 & 366 & 486 & 140 & 139 \\
\hline $5^{2}$ & 43.042 & 83.094 & 378.137 & 602.461 & 79.725 & 58.124 & 789.652 & 669.803 \\
\hline$m$ & \multicolumn{2}{|c|}{840} & \multicolumn{2}{|c|}{265} & \multicolumn{2}{|c|}{850} & \multicolumn{2}{|c|}{277} \\
\hline$t^{*}$ & \multicolumn{2}{|c|}{1,82} & \multicolumn{2}{|c|}{2,73} & \multicolumn{2}{|c|}{0,50} & \multicolumn{2}{|c|}{1,16} \\
\hline t & \multicolumn{2}{|c|}{1,65} & \multicolumn{2}{|c|}{1,65} & \multicolumn{2}{|c|}{1,65} & \multicolumn{2}{|c|}{1,65} \\
\hline $\begin{array}{l}\text { Unterschied } \\
\text { signifikant? }\end{array}$ & \multicolumn{2}{|c|}{ ja } & \multicolumn{2}{|c|}{ ja } & \multicolumn{2}{|c|}{ nein } & \multicolumn{2}{|c|}{ nein } \\
\hline \multicolumn{9}{|c|}{ nur Unterschreitungen (in \% des Sicherheitsabstandes $1=50 \%$ der gefahrenen Geschwindigkeit) } \\
\hline $\begin{array}{l}\text { Anteil an allen } \\
\text { Lücken [\%] }\end{array}$ & 44 & 49 & 31 & 23 & 37 & 35 & 21 & 23 \\
\hline $\begin{array}{l}\text { Mittelwert } \\
{[\%]}\end{array}$ & 61,7 & 62,0 & 54,3 & 55,6 & 60,6 & 65,9 & 45,6 & 53,1 \\
\hline Anzahl & 197 & 197 & 45 & 28 & 140 & 175 & 30 & 32 \\
\hline$s^{2}$ & 419 & 377 & 433 & 491 & 323 & 306 & 359 & 369 \\
\hline$m$ & \multicolumn{2}{|c|}{392} & \multicolumn{2}{|c|}{71} & \multicolumn{2}{|c|}{313} & \multicolumn{2}{|c|}{60} \\
\hline$t^{*}$ & \multicolumn{2}{|c|}{0.15} & \multicolumn{2}{|c|}{0,24} & \multicolumn{2}{|c|}{2,61} & \multicolumn{2}{|c|}{1,55} \\
\hline t & \multicolumn{2}{|c|}{1,65} & & & & & & \\
\hline $\begin{array}{l}\text { Unterschied } \\
\text { signifikant? }\end{array}$ & & & & & & & & \\
\hline $\begin{array}{l}s_{n} \text { in }[\mathrm{m}] \\
\text { bei } 80 \mathrm{~km} / \mathrm{h}\end{array}$ & 25 & 25 & 22 & 22 & 24 & 26 & 18 & 21 \\
\hline
\end{tabular}

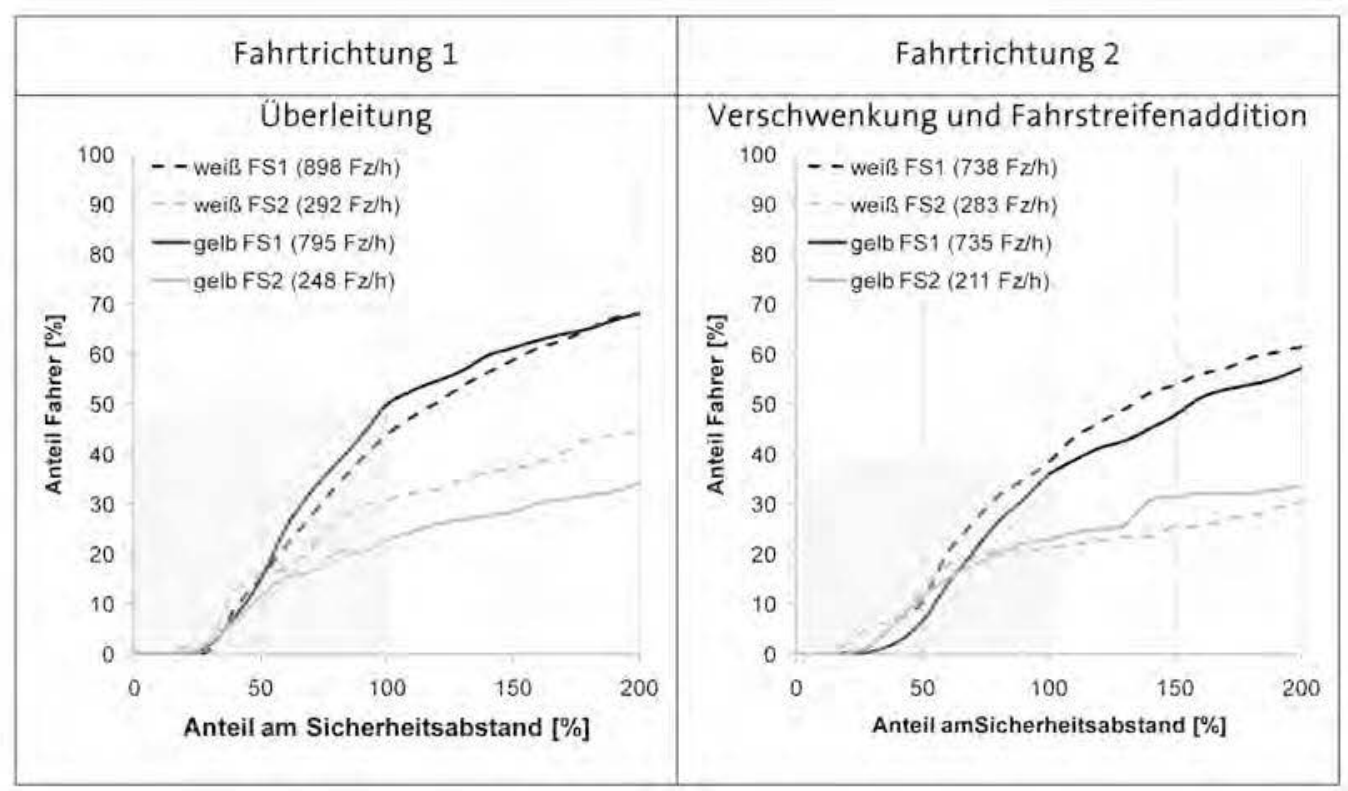


Baustellenende -

Nach der Fahrstreifenverschwenkung (100 km/h)

\begin{tabular}{|c|c|c|c|c|c|c|c|c|}
\hline & \multicolumn{4}{|c|}{ Fahrtrichtung 1} & \multicolumn{4}{|c|}{ Fahrtrichtung 2} \\
\hline & \multicolumn{2}{|c|}{ FS1 } & \multicolumn{2}{|c|}{ FS2 } & \multicolumn{2}{|c|}{ FS1 } & \multicolumn{2}{|c|}{ FS2 } \\
\hline & weiß & gelb & weißs & gelb & weiß & gelb & weiß & gelb \\
\hline \multicolumn{9}{|c|}{ alle Zeitlücken (in \% des Sicherheitsabstandes I = 50\% der gefahrenen Geschwindigkeit) } \\
\hline $\operatorname{Min}[\%]$ & 20 & 9 & 13 & 13 & 22 & 22 & 22 & 16 \\
\hline $\operatorname{Max}[\%]$ & 1.640 & 1.916 & 3.262 & 3.278 & 2.107 & 1,489 & 3.198 & 3.280 \\
\hline $\begin{array}{l}\text { Mittelwert } \\
{[\%]}\end{array}$ & 219,3 & 268,8 & 555,2 & 829,9 & 224,6 & 235,4 & 758,7 & 977,6 \\
\hline Anzahl & 404 & 338 & 194 & 132 & 406 & 363 & 157 & 140 \\
\hline $5^{2}$ & 55.444 & 87.967 & 487,570 & 654.335 & 65.856 & 66.763 & 718.231 & 895.903 \\
\hline$m$ & \multicolumn{2}{|c|}{740} & \multicolumn{2}{|c|}{324} & \multicolumn{2}{|c|}{767} & \multicolumn{2}{|c|}{295} \\
\hline$t^{*}$ & \multicolumn{2}{|c|}{2,53} & \multicolumn{2}{|c|}{3,27} & \multicolumn{2}{|c|}{0,58} & \multicolumn{2}{|c|}{2,10} \\
\hline t & \multicolumn{2}{|c|}{1,65} & \multicolumn{2}{|c|}{1,65} & \multicolumn{2}{|c|}{1.65} & \multicolumn{2}{|c|}{1,65} \\
\hline $\begin{array}{l}\text { Unterschied } \\
\text { signifikant? }\end{array}$ & \multicolumn{2}{|c|}{ ja } & \multicolumn{2}{|c|}{ ja } & \multicolumn{2}{|c|}{ nein } & \multicolumn{2}{|c|}{ ja } \\
\hline \multicolumn{9}{|c|}{ nur Unterschreitungen (in \% des Sicherheitsabstandes I = 50\% der gefahrenen Geschwindigkeit) } \\
\hline $\begin{array}{l}\text { Anteil an allen } \\
\text { Lücken [\%] }\end{array}$ & 39 & 36 & 30 & 14 & 41 & 39 & 25 & 19 \\
\hline $\begin{array}{l}\text { Mittelwert } \\
{[\%]}\end{array}$ & 62,6 & 62,5 & 48,9 & 56,2 & 60,4 & 61,9 & 54,0 & 48,4 \\
\hline Anzahl & 160 & 124 & 58 & 18 & 172 & 145 & 39 & 27 \\
\hline$s^{2}$ & 387 & 447 & 403 & 553 & 468 & 396 & 265 & 509 \\
\hline$m$ & \multicolumn{2}{|c|}{282} & \multicolumn{2}{|c|}{74} & \multicolumn{2}{|c|}{315} & \multicolumn{2}{|c|}{64} \\
\hline$t^{*}$ & \multicolumn{2}{|c|}{0,04} & \multicolumn{2}{|c|}{1,29} & \multicolumn{2}{|c|}{0,65} & \multicolumn{2}{|c|}{1,16} \\
\hline t & \multicolumn{2}{|c|}{1,65} & & & & & & \\
\hline $\begin{array}{l}\text { Unterschied } \\
\text { signifikant? }\end{array}$ & & & & & & & & \\
\hline $\begin{array}{l}\mathrm{s}_{\mathrm{e}} \text { in }[\mathrm{m}] \\
\text { bei } 80 \mathrm{~km} / \mathrm{h}\end{array}$ & 25 & 25 & 20 & 22 & 24 & 25 & 22 & 19 \\
\hline
\end{tabular}

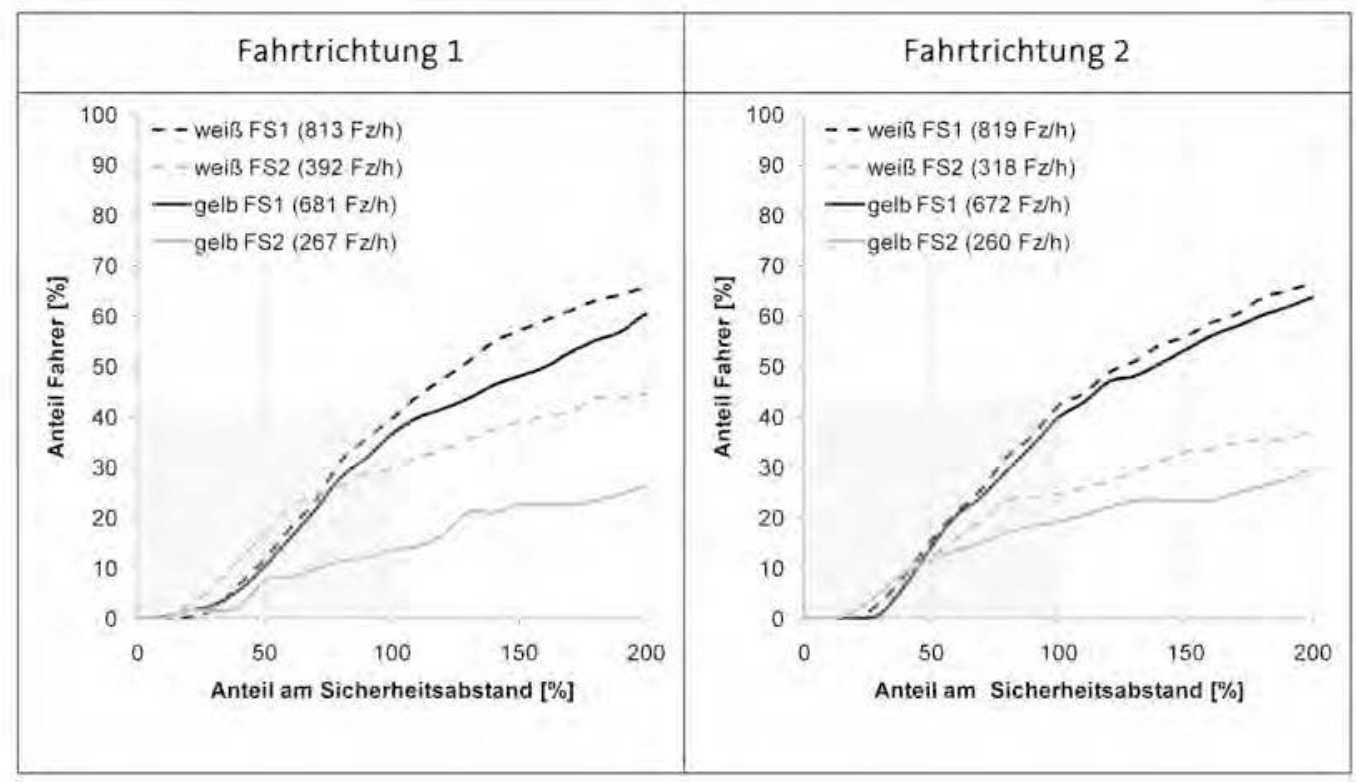




\section{Baustellenende -}

Aufhebung der baustellenbedingten Streckenverbote

\begin{tabular}{|c|c|c|c|c|c|c|c|c|}
\hline & \multicolumn{4}{|c|}{ Fahrtrichtung 1} & \multicolumn{4}{|c|}{ Fahrtrichtung 2} \\
\hline & \multicolumn{2}{|c|}{ FS1 } & \multicolumn{2}{|c|}{ FS2 } & \multicolumn{2}{|c|}{ FS1 } & \multicolumn{2}{|c|}{ FS2 } \\
\hline & weiß & gelb & weiß & gelb & weiß & gelb & weiß & gelb \\
\hline \multicolumn{9}{|c|}{ alle Zeitlücken (in \% des Sicherheitsabstandes I = 50\% der gefahrenen Geschwindigkeit) } \\
\hline $\operatorname{Min}[\%]$ & 20 & 29 & 27 & 16 & 22 & 20 & 16 & 24 \\
\hline $\operatorname{Max}[\%]$ & 3.002 & 1.602 & 3.147 & 3.284 & 1,524 & 2.111 & 3.069 & 2.969 \\
\hline $\begin{array}{l}\text { Mittelwert } \\
{[\%]}\end{array}$ & 316,9 & 301,5 & 591,5 & 609,6 & 292,7 & 321,4 & 533,6 & 457,3 \\
\hline Anzahl & 297 & 306 & 173 & 193 & 294 & 269 & 171 & 179 \\
\hline$s^{2}$ & 127.856 & 93.455 & 507.188 & 546,874 & 71.822 & 109.463 & 412.511 & 294.182 \\
\hline m & \multicolumn{2}{|c|}{601} & \multicolumn{2}{|c|}{364} & \multicolumn{2}{|c|}{561} & \multicolumn{2}{|c|}{348} \\
\hline$t^{*}$ & \multicolumn{2}{|c|}{0,57} & \multicolumn{2}{|c|}{0,24} & \multicolumn{2}{|c|}{1,14} & \multicolumn{2}{|c|}{1,20} \\
\hline $\mathrm{t}$ & \multicolumn{2}{|c|}{1,65} & \multicolumn{2}{|c|}{1,65} & \multicolumn{2}{|c|}{1,65} & \multicolumn{2}{|c|}{1,65} \\
\hline $\begin{array}{l}\text { Unterschied } \\
\text { signifikant? }\end{array}$ & \multicolumn{2}{|c|}{ nein } & \multicolumn{2}{|c|}{ nein } & \multicolumn{2}{|c|}{ nein } & \multicolumn{2}{|c|}{ nein } \\
\hline \multicolumn{9}{|c|}{ nur Unterschreitungen (in \% des Sicherheitsabstandes I = 50\% der gefahrenen Geschwindigkeit) } \\
\hline $\begin{array}{l}\text { Anteil an allen } \\
\text { Lücken [\%] }\end{array}$ & 26 & 29 & 25 & 32 & 25 & 27 & 29 & 31 \\
\hline $\begin{array}{l}\text { Mittelwert } \\
{[\%]}\end{array}$ & 61,0 & 67,9 & 63,8 & 52,4 & 65,4 & 64,6 & 57,7 & 65,4 \\
\hline Anzahl & 81 & 91 & 43 & 61 & 74 & 73 & 49 & 55 \\
\hline $5^{2}$ & 441 & 363 & 435 & 431 & 402 & 386 & 603 & 424 \\
\hline $\mathrm{m}$ & \multicolumn{2}{|c|}{170} & \multicolumn{2}{|c|}{102} & \multicolumn{2}{|c|}{145} & \multicolumn{2}{|c|}{102} \\
\hline$t^{*}$ & \multicolumn{2}{|c|}{2,24} & \multicolumn{2}{|c|}{2,74} & \multicolumn{2}{|c|}{0,26} & \multicolumn{2}{|c|}{1,74} \\
\hline t & \multicolumn{2}{|c|}{1,66} & & & & & & \\
\hline $\begin{array}{l}\text { Unterschied } \\
\text { signifikant? }\end{array}$ & & & & & & & & \\
\hline $\begin{array}{l}s_{\mathrm{x}} \text { in }[\mathrm{m}] \\
\text { bei } 80 \mathrm{~km} / \mathrm{h}\end{array}$ & 24 & 27 & 26 & 21 & 26 & 26 & 23 & 26 \\
\hline
\end{tabular}

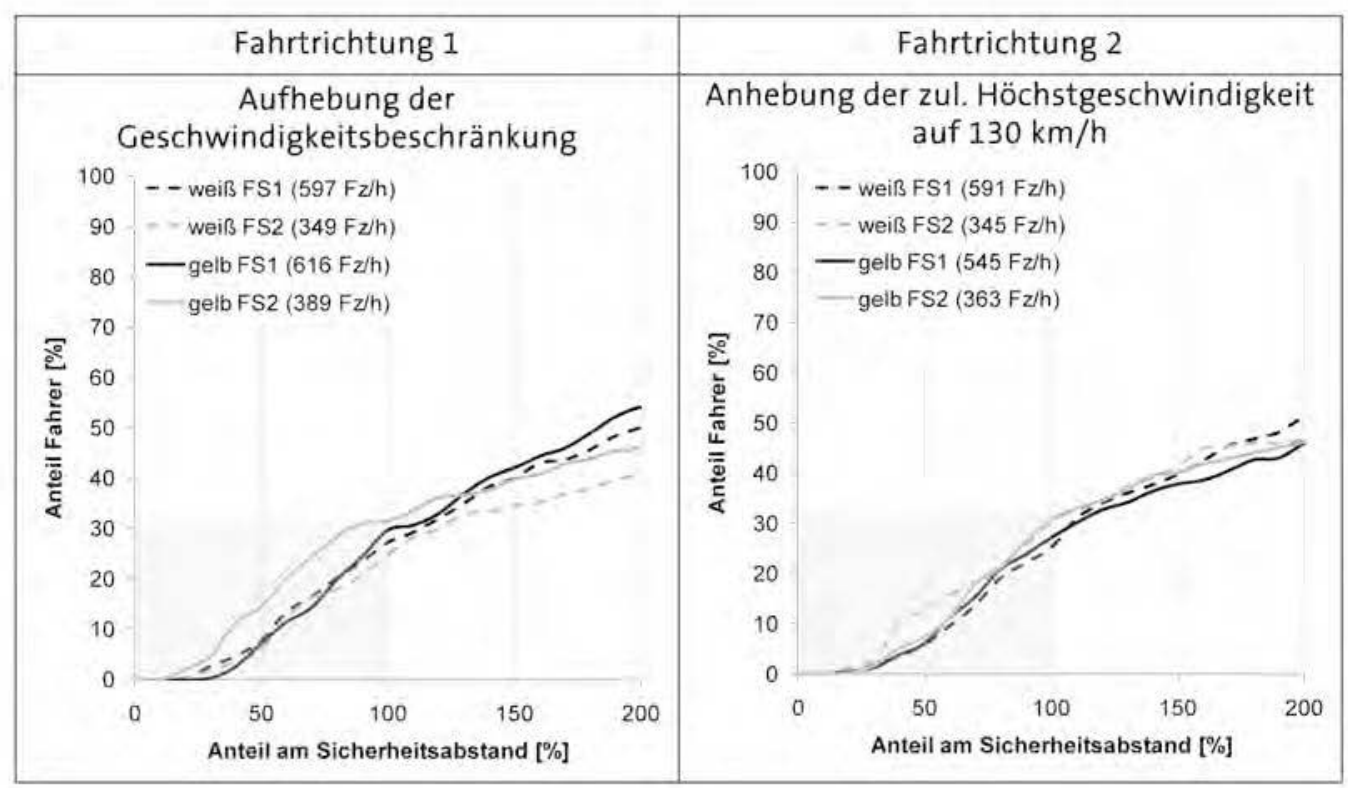




\section{E.5 Anschlussstelle}

\begin{tabular}{|c|c|c|c|c|c|c|c|c|}
\hline & \multicolumn{4}{|c|}{$200 \mathrm{~m}$ vor Anschlussstelle } & \multicolumn{4}{|c|}{ Beginn Verzögerungsstreifen } \\
\hline & \multicolumn{2}{|c|}{ FS1 } & \multicolumn{2}{|c|}{ FS2 } & \multicolumn{2}{|c|}{ FS1 } & \multicolumn{2}{|c|}{ FS2 } \\
\hline & weiß & gelb & weiß & gelb & weiß & gelb & weiß & gelb \\
\hline \multicolumn{9}{|c|}{ alle Zeitlücken (in \% des Sicherheitsabstandes I = 50\% der gefahrenen Geschwindigkeit) } \\
\hline $\operatorname{Min}[\%]$ & 29 & 20 & 9 & 31 & 22 & 27 & 20 & 24 \\
\hline $\operatorname{Max}[\%]$ & 1.564 & 2.011 & 3.327 & 3.113 & 1.698 & 1.713 & 3.016 & 3.224 \\
\hline $\begin{array}{l}\text { Mittelwert } \\
{[\%]}\end{array}$ & 234,3 & 257,6 & 669,0 & 702,2 & 238,2 & 242,1 & 599,0 & 682,1 \\
\hline Anzahl & 512 & 300 & 123 & 76 & 505 & 363 & 127 & 113 \\
\hline$s^{2}$ & 57.133 & 101.558 & 683.486 & 616.855 & 67.025 & 71.984 & 575.872 & 569.332 \\
\hline$m$ & \multicolumn{2}{|c|}{810} & \multicolumn{2}{|c|}{197} & \multicolumn{2}{|c|}{866} & \multicolumn{2}{|c|}{238} \\
\hline$t^{*}$ & \multicolumn{2}{|c|}{1,18} & \multicolumn{2}{|c|}{0,28} & \multicolumn{2}{|c|}{0,22} & \multicolumn{2}{|c|}{0,85} \\
\hline t & \multicolumn{2}{|c|}{1,65} & \multicolumn{2}{|c|}{1,66} & \multicolumn{2}{|c|}{1,65} & \multicolumn{2}{|c|}{1,65} \\
\hline $\begin{array}{l}\text { Unterschied } \\
\text { signifikant? }\end{array}$ & \multicolumn{2}{|c|}{ nein } & \multicolumn{2}{|c|}{ nein } & \multicolumn{2}{|c|}{ nein } & \multicolumn{2}{|c|}{ nein } \\
\hline \multicolumn{9}{|c|}{ nur Unterschreitungen (in \% des Sicherheitsabstandes I = 50\% der gefahrenen Geschwindigkeit) } \\
\hline $\begin{array}{l}\text { Anteil an allen } \\
\text { Lücken [\%] }\end{array}$ & 34 & 38 & 33 & 25 & 37 & 35 & 33 & 26 \\
\hline $\begin{array}{l}\text { Mittelwert } \\
{[\%]}\end{array}$ & 68,0 & 68,9 & 54,7 & 58,1 & 64,9 & 66,9 & 59,1 & 61,0 \\
\hline Anzahl & 182 & 119 & 40 & 19 & 188 & 130 & 42 & 29 \\
\hline$s^{2}$ & 324 & 342 & 418 & 272 & 356 & 339 & 432 & 350 \\
\hline$m$ & \multicolumn{2}{|c|}{299} & \multicolumn{2}{|c|}{57} & \multicolumn{2}{|c|}{316} & \multicolumn{2}{|c|}{69} \\
\hline$t^{*}$ & \multicolumn{2}{|c|}{0,42} & \multicolumn{2}{|c|}{0,64} & \multicolumn{2}{|c|}{0,94} & \multicolumn{2}{|c|}{0,39} \\
\hline t & \multicolumn{2}{|c|}{1,65} & & & & & & \\
\hline $\begin{array}{l}\text { Unterschied } \\
\text { signifikant? }\end{array}$ & & & & & & & & \\
\hline $\begin{array}{l}\mathrm{s}_{\mathrm{e}} \text { in }[\mathrm{m}] \\
\text { bei } 80 \mathrm{~km} / \mathrm{h}\end{array}$ & 27 & 28 & 22 & 23 & 26 & 27 & 24 & 24 \\
\hline
\end{tabular}

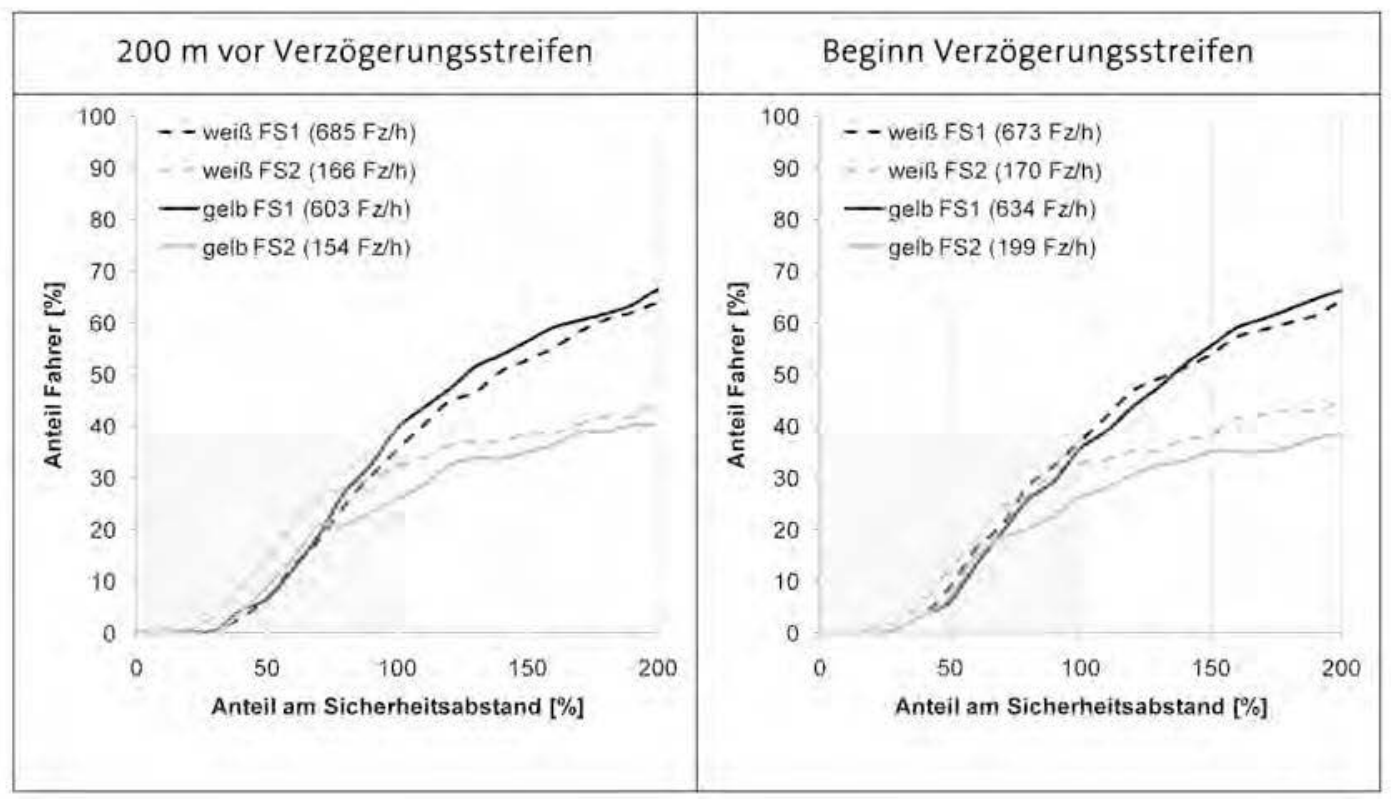




\section{F Fahrstreifenbelegung}

\section{F.1 Baustellenbeginn}

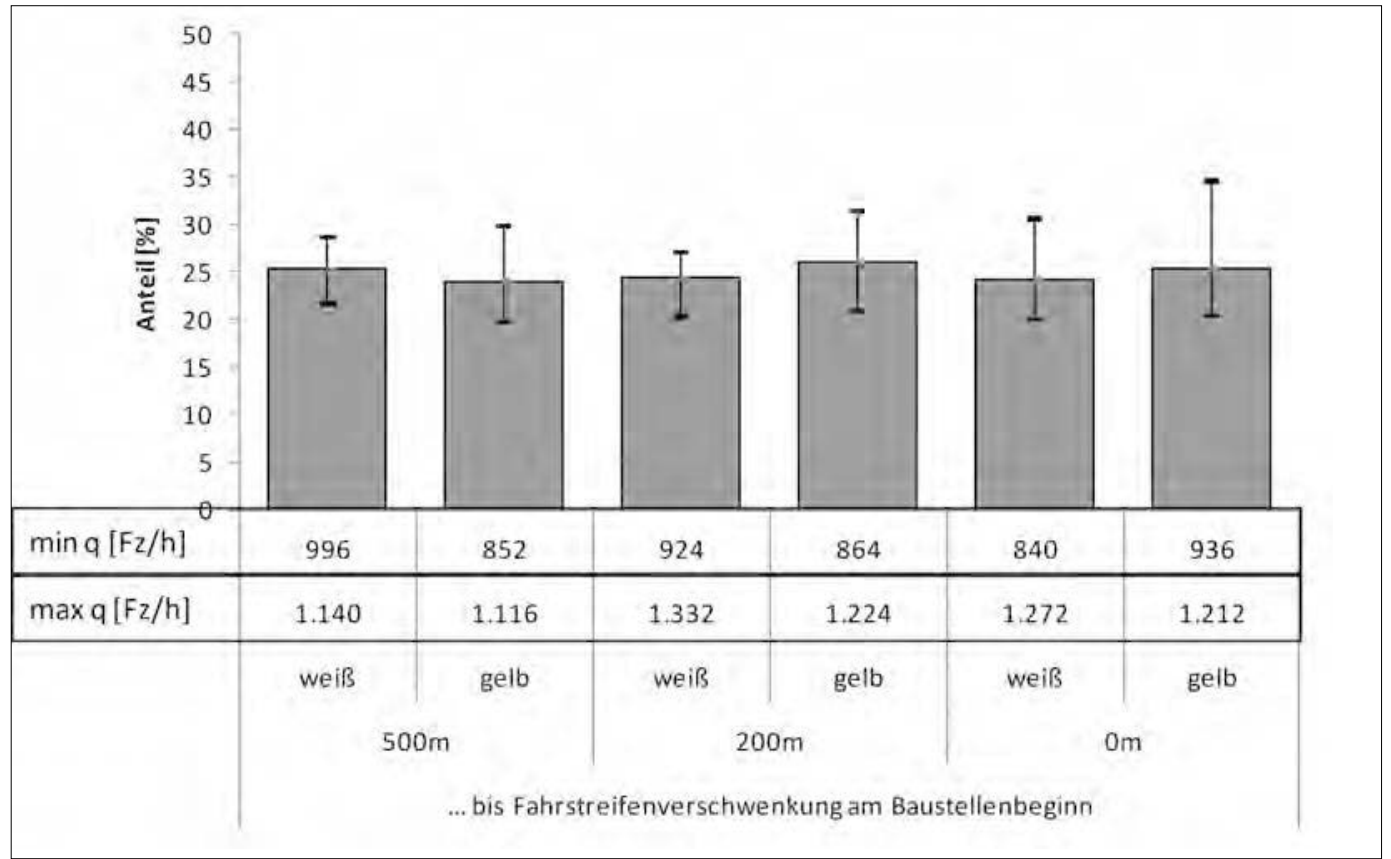

Abbildung F-1:

Belegung des Überholfahrstreifens an den Messquerschnitten im Annäherungsbereich im Beobachtungszeitraum - Fahrtrichtung 1

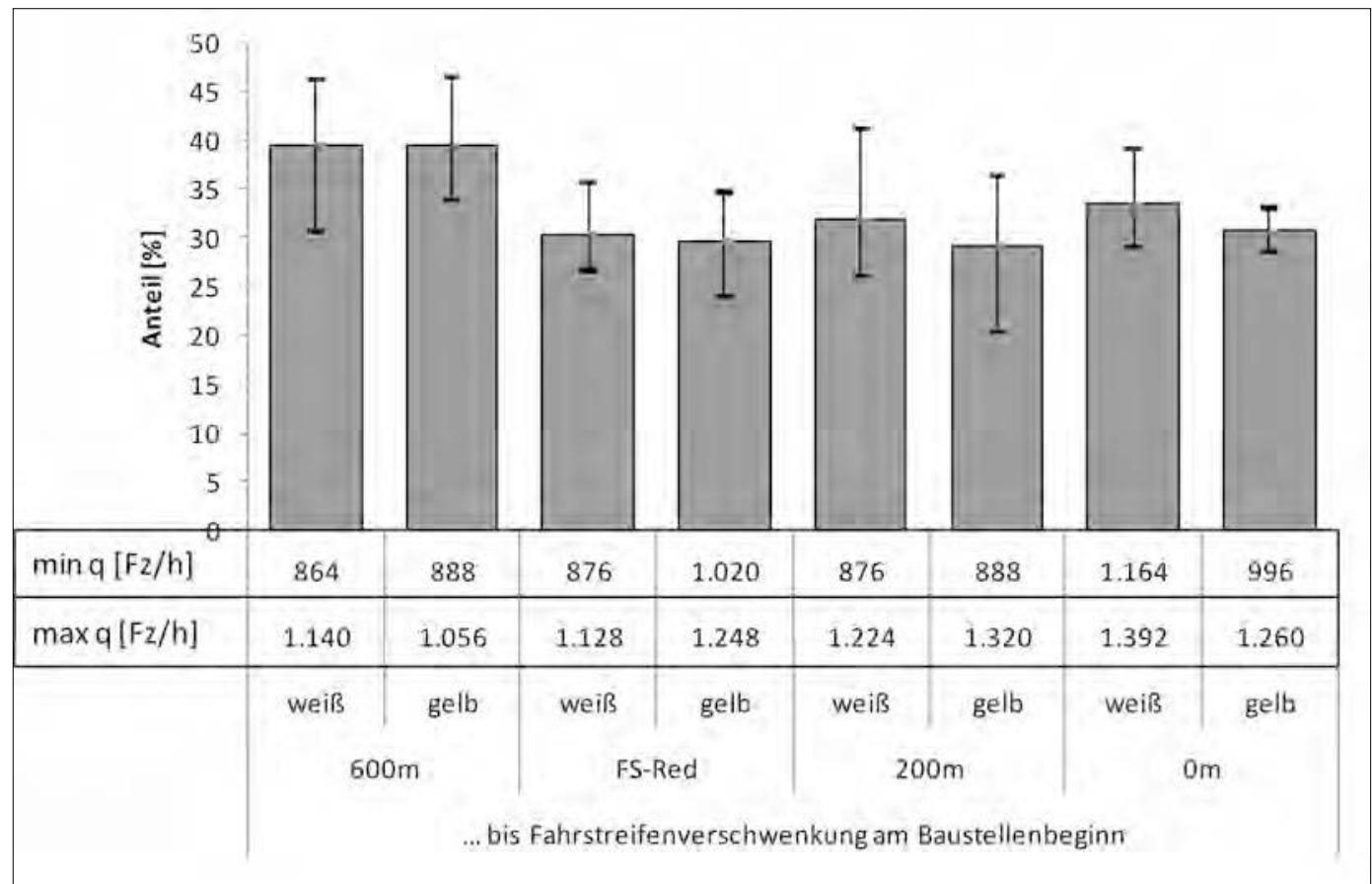

Abbildung F-2: Belegung des Überholfahrstreifens an den Messquerschnitten im Annäherungsbereich im Beobachtungszeitraum - Fahrtrichtung 2 


\section{F.2 Baustellenende}

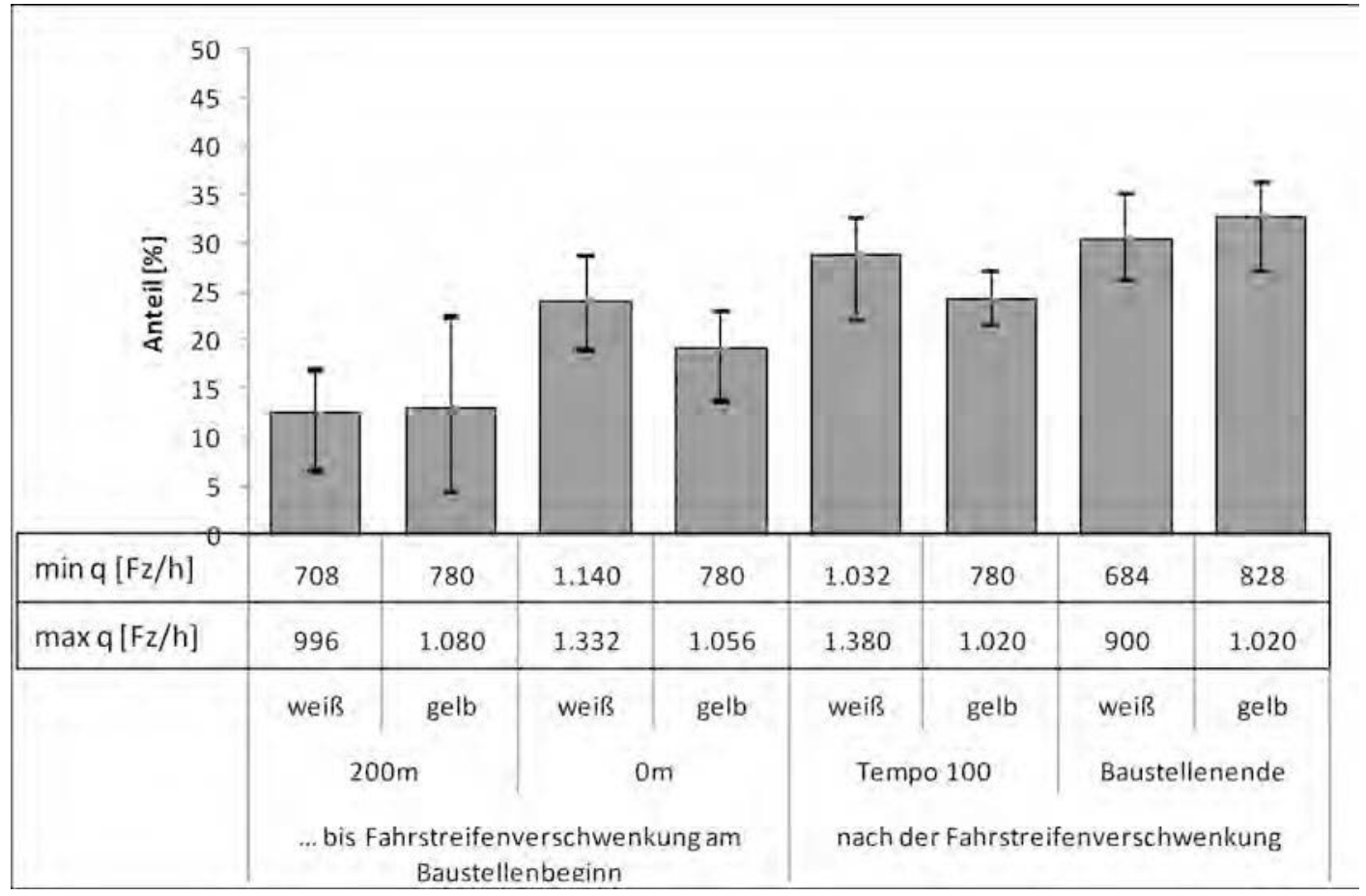

Abbildung F-3:

Belegung des Überholfahrstreifens an den Messquerschnitten am Baustellenende im Beobachtungszeitraum - Fahrtrichtung 1

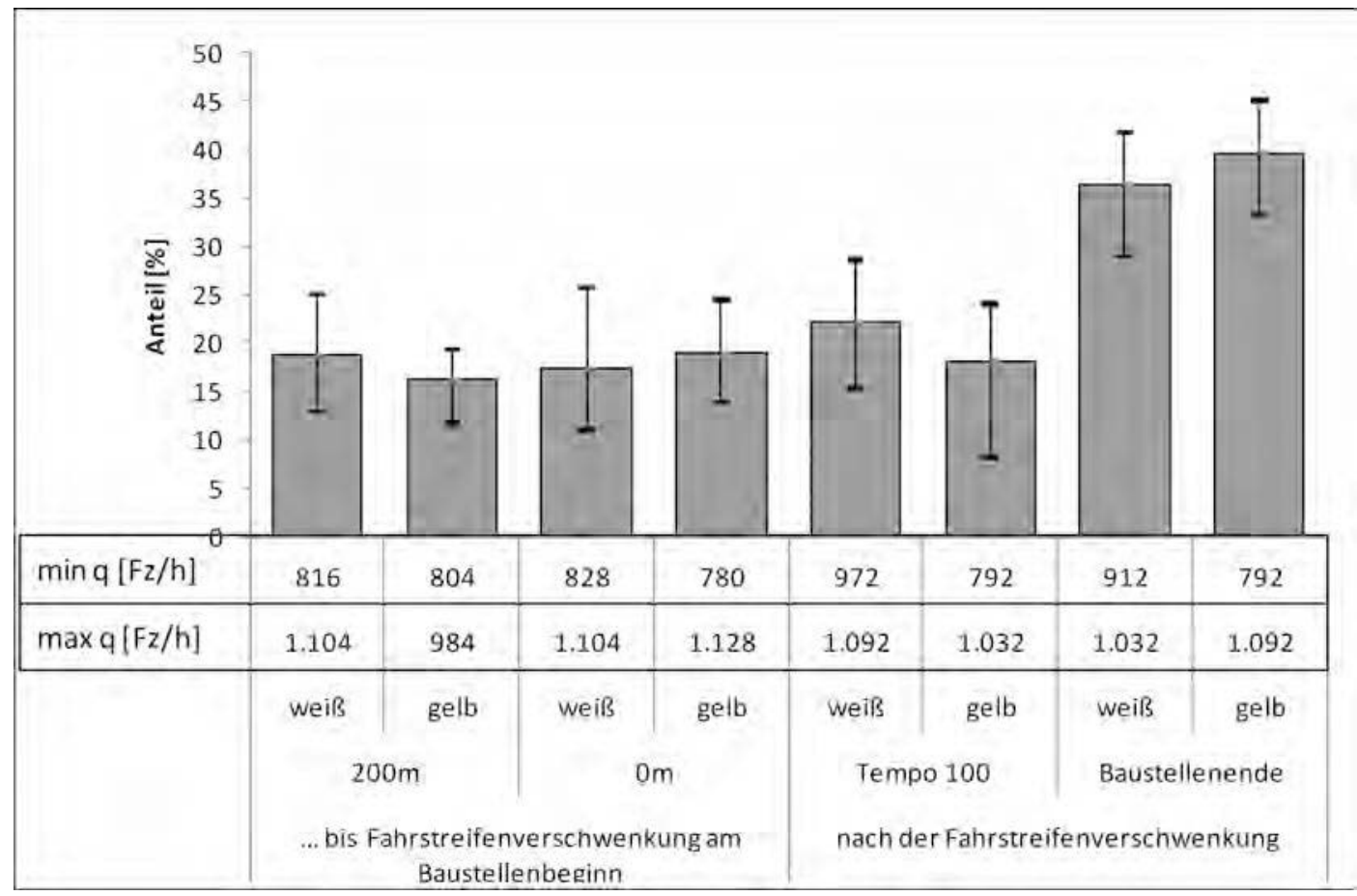

Abbildung F-4:

Belegung des Überholfahrstreifens an den Messquerschnitten am Baustellenende im Beobachtungszeitraum - Fahrtrichtung 2 


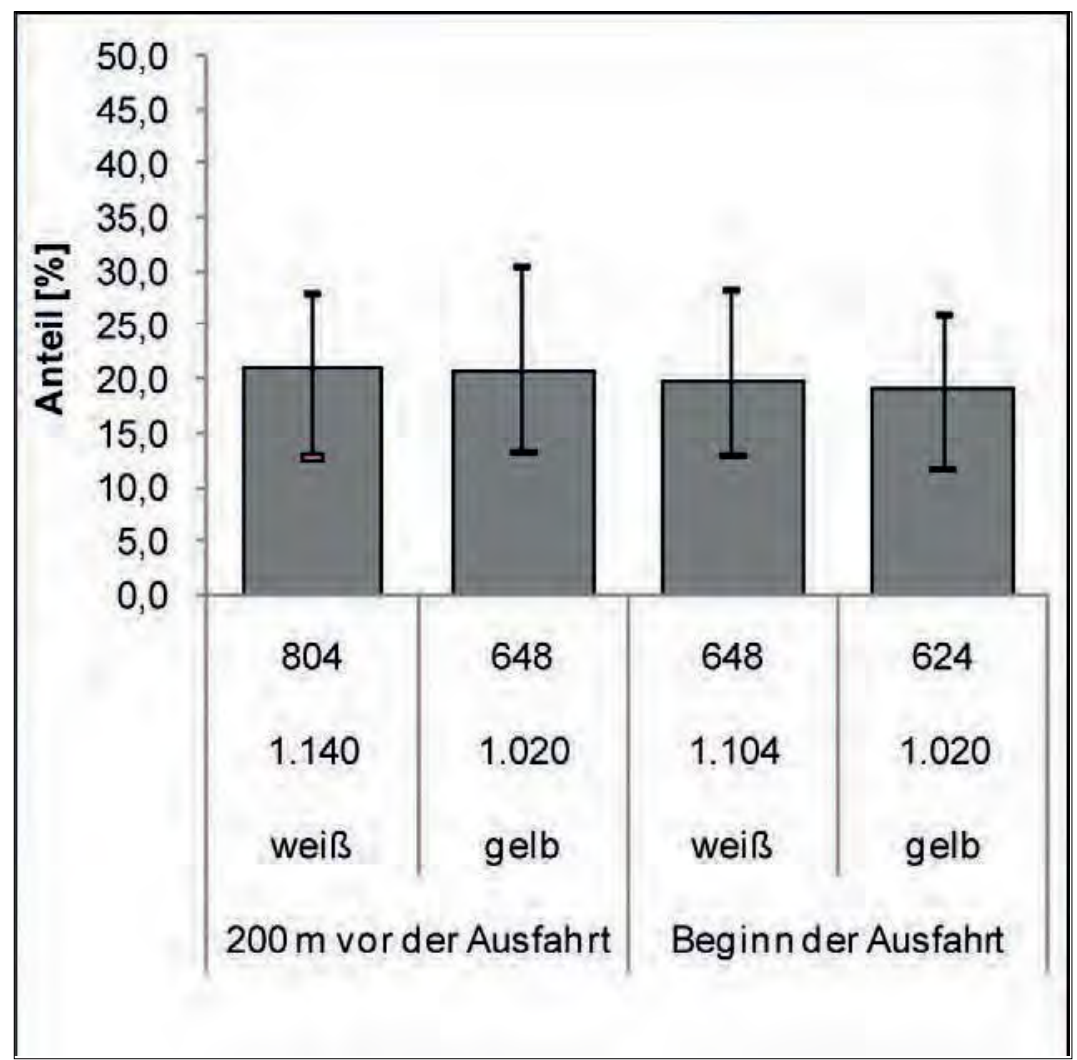

Abbildung F-5:

Belegung des Überholfahrstreifens an den Messquerschnitten vor der Behelfsausfahrt im Beobachtungszeitraum - Fahrtrichtung 2 


\section{G Unfallgeschehen}

Im Bereich der Autobahnbaustelle ${ }^{3}$ ereigneten sich im Untersuchungszeitraum 23 Unfälle. 10 dieser Unfälle ereigneten sich in Zeiten der Einrichtung oder des Abbaus der Baustelle und 13 Unfälle während des Bauzeitraums.

Kein Unfall hatte einen Personenschaden zur Folge, zwei ereigneten sich mit schwerem Sachschaden. Die Unfallrate, welche aufgrund des geringen zu Grunde liegenden Unfallkollektivs keine statistische Aussagekraft besitzt, liegt im Bereich des in ENKE et al. (2010) ermittelten Wertes für Baustellen auf Autobahnen (0,9 U/106 Kfz*km). Diese Unfallkostenraten lagen deutlich unter den Werten deutlich unter den Werten der früheren Untersuchung.

Insgesamt ereigneten sich 9 der 23 Unfälle auf nasser bzw. winterglatter Fahrbahn bei Dunkelheit. Allerdings sind diese Zahlen vor dem Hintergrund des Bauzeitraums, welcher sich von September bis Dezember erstreckte, zu bewerten. Die Unfälle bei Nässe und Dunkelheit treten erst im letzten Untersuchungsmonat, welcher durch häufige Regenfälle und Wintereinbruch gekennzeichnet war, auf. Insbesondere aus diesem Zeitraum resultiert die Verschlechterung der Verkehrssicherheit der modifi-

\section{Tabelle G-1:}

Ausprägung der Unfallumstände in der untersuchten Baustelle im Vergleich zu ENKE et al. (2010)

\begin{tabular}{|l|c|c|c|}
\hline & $\begin{array}{c}\text { Autobah } \\
\mathrm{n}- \\
\text { baustelle }\end{array}$ & \multicolumn{2}{|c|}{ Fahrtrichtung } \\
\hline Unfallanzahl & & 13 & 10 \\
\hline Wochenende & $17 \%$ & $23 \%$ & $0 \%$ \\
\hline Spitzenstunde & $39 \%$ & $31 \%$ & $20 \%$ \\
\hline $\begin{array}{l}\text { Dämmerung / } \\
\text { Dunkelheit }\end{array}$ & $33 \%$ & $54 \%$ & $40 \%$ \\
\hline Nässe / Glätte & $19 \%$ & $54 \%$ & $70 \%$ \\
\hline
\end{tabular}

zierten Baustelle gegenüber der Gestaltung nach RSA 95. Betrachtet man ausschließlich die Zeitbereiche, in denen die Baustelle unverändert mit der gleichen Beschilderung und Verkehrsführung bestand, traten bei der Gestaltung nach RSA 95 drei und im Zeitraum der gelben Beschilderung 10 Unfälle auf.

Fünf der 13 Unfälle im unveränderten Baustellenbereich auf den freien Strecken innerhalb der Baustelle ereigneten (Tabelle G 2), davon:

- zwei Fahrunfälle (Unfalltyp 1),

- zwei Unfälle im Längsverkehr (Unfalltyp 6)

und

- ein Sonstiger Unfall (Unfalltyp 7).

Die beiden Unfälle im Längsverkehr ereigneten sich beim Überholen und wurden jeweils durch den rechts fahrenden Lkw verursacht. Bei den Fahrunfällen lag in einem Fall ein technischer Defekt des Fahrzeugs vor, welcher zum Abkommen von der Fahrbahn führte.

Die vier Unfälle in den Annäherungsbereichen der Baustelle wurden zu gleichen Teilen den Unfalltypen 6 (Fahrunfall) und 7 (Sonstiger Unfall) zugeordnet und hervorgerufen durch:

\section{Tabelle G-2:}

Unfälle nach Abschnitten der untersuchten Baustelle im baulich unveränderten Zeitraum

\begin{tabular}{|c|c|c|c|c|c|}
\hline \multirow{2}{*}{\multicolumn{2}{|c|}{$\begin{array}{l}\text { Beschilderung } \\
\text { Fahrtrichtung }\end{array}$}} & \multicolumn{2}{|c|}{ weiß } & \multicolumn{2}{|c|}{ gelb } \\
\hline & & 1 & 2 & 1 & 2 \\
\hline \multicolumn{2}{|c|}{ Unfallanzahl } & 2 & 1 & 5 & 5 \\
\hline \multirow{5}{*}{ 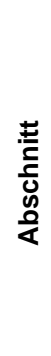 } & Annäherung & & 1 & 3 & \\
\hline & $\begin{array}{l}\text { Fahrstreifenverschwenkung } \\
\text { am Baustellenbeginn }\end{array}$ & & & 1 & 1 \\
\hline & Strecke & 2 & & 1 & 2 \\
\hline & $\begin{array}{l}\text { Fahrstreifenverschwenkung } \\
\text { am Baustellenende }\end{array}$ & & & & \\
\hline & Nachlauf (500 m) & & & & 2 \\
\hline
\end{tabular}

3 Es wurden jeweils die gesamten Bereiche zwischen dem ersten Z 123 (Baustelle) und der Aufhebung aller mit der Baustelle in Verbindung stehenden Streckenverbote (zulässige Höchstgeschwindigkeit und Überholverbot) betrachtet. 
- Zusammenstoß mit einem Fahrzeug, das vorausfährt oder wartet (Unfalltyp 6, Unfallart 2) aufgrund von Stau, - Einen Fahrstreifenwechsel vom rechten auf den linken Fahrstreifen (Unfalltyp 6, Unfallart 3),

- Abkommen von der Fahrbahn nach links im Bereich der Fahrstreifenreduzierung (Unfalltyp 7, Unfallart 9) und - Unfall durch Wild (Unfalltyp 7, Unfallart 7).

Nur der Unfall mit Abkommen von der Fahrbahn nach links im Bereich der Fahrstreifenverschwenkung passierte während der Phase der weißen Beschilderung. Allerdings ereignete sich keiner der Unfälle im Zeitraum der gelben Beschilderung im Wirkungsbereich der modifizierten Verkehrszeichen.

Beide Unfälle im Bereich der Fahrstreifenverschwenkungen am Beginn der Baustellen passierten währen der gelben Ausstattung dieser Bereiche. Während bei dem Unfall in der Überleitung (Fahrtrichtung 1) der überholende Transporter den Unfall mit einem Lkw verursachte, wurde die Kollision zwischen zwei Pkw in der Verschwenkung der Gegenrichtung durch einen Fahrstreifenwechsel nach links hervorgerufen. 


\section{GDV}

DIE DEUTSCHEN VERSICHERER

\section{Gesamtverband der Deutschen Versicherungswirtschaft e.V.}

Wilhelmstraße 43 / 43 G, 10117 Berlin

Postfach 0802 64, 1002 Berlin

Tel. 030 / 2020 -50 00, Fax 030 / 2020 - 6000

E-Mai: unfallforschung@gdv.de

www.gdv.de, www.udv.de 